\title{
Untersuchung atomarer Strukturen an geordnetem und nanokristallinem ternären $\mathrm{Fe}_{3} \mathrm{Al}$
}

\author{
Dissertation \\ zur Erlangung des mathematisch-naturwissenschaftlichen Doktorgrades \\ „Doctor rerum naturalium“ \\ der Georg-August-Universität Göttingen
}

vorgelegt von

Thomas Rademacher

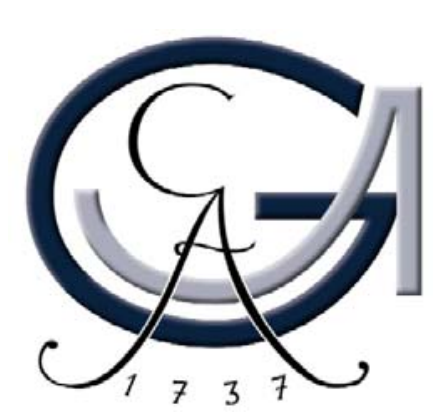

Göttingen 2011 
Untersuchung atomarer Strukturen an

geordnetem und nanokristallinem ternären $\mathrm{Fe}_{3} \mathrm{Al}$

Dissertation

vorgelegt von

Thomas Rademacher

aus Lehrte

angefertigt am Institut für Materialphysik

Georg-August-Universität Göttingen

Juni 2011

Referent: Prof. Dr. Reiner Kirchheim

Koreferent: Prof. Dr. Talaat Al-Kassab

Tag der mündlichen Prüfung: 13.7.2011 


\section{Inhalt}

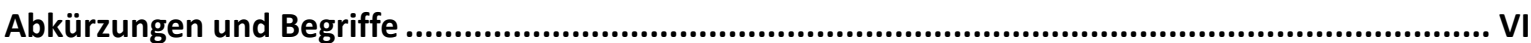

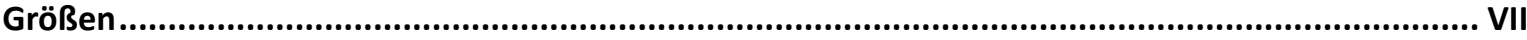

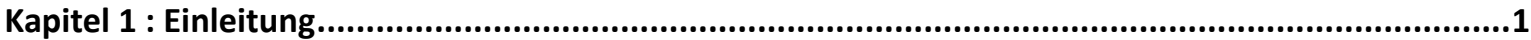

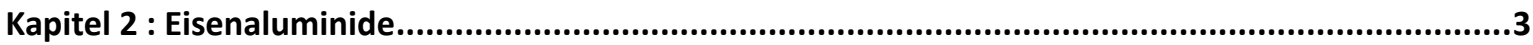

2.1. Phasen und mechanische Eigenschaften ......................................................................... 3

2.2. Platzbesetzung und Ordnung ternärer Elemente ............................................................ 5

2.3. Herstellung und Eigenschaften nanokristallinen Gefüges ..................................................... 7

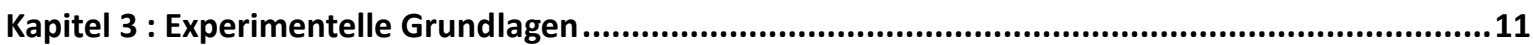

3.1. Atomsondentomographie und Feldionenmikroskopie ....................................................... 11

3.1.1. Aufbau und Arbeitsprinzip 11

3.1.2. Auswertungsmethoden der Atomsondentomographie 13

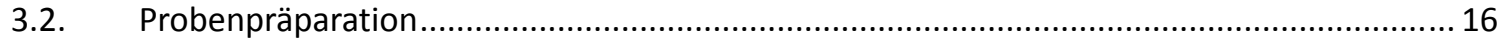

$\begin{array}{ll}\text { 3.2.1. Geordnete, massive Materialen } & 16\end{array}$

$\begin{array}{ll}\text { 3.2.2. Nanokristalline Materialien } & 17\end{array}$

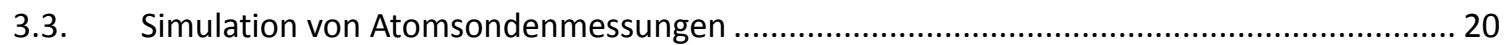

3.3.1. Allgemeines Arbeitsprinzip 20

3.3.2. Berechnung der Aktivierungsenergie $Q \quad 20$

3.3.3. Berechnung der Bindungsenergie mithilfe der Embedded Atom Method 23

3.3.4. Weitere Neuerungen des Simulationsprogramms 24

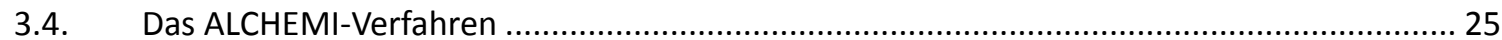

Kapitel 4 : Allgemeine Analyse von FIM- und APT-Messungen .........................................................27

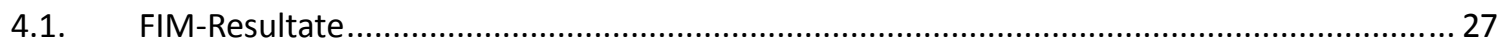

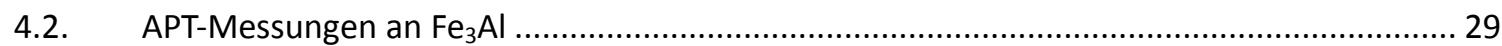

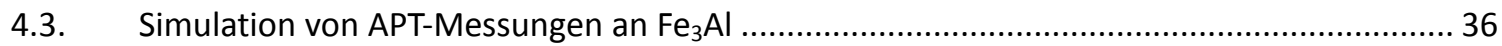

4.3.1. Optimierung der Parameter 36

4.3.2. Analyse der Simulationen 36

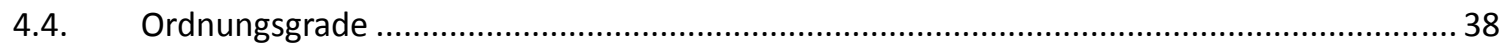

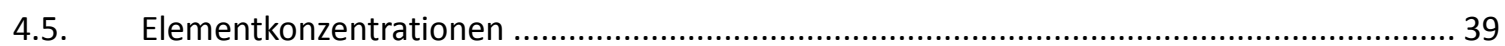

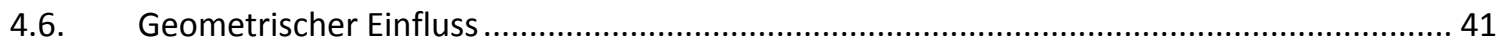

Kapitel 5 : Bestimmung der Platzbesetzung mittels APT-Messungen und -Simulationen .....................43

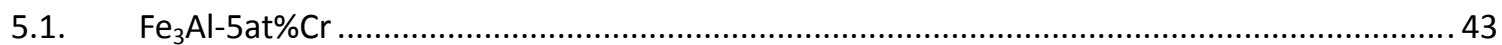

5.1.1. Analyse der AtomVicinity-Dichteprofile 43

5.1.2. Simulationen der Messungen 45

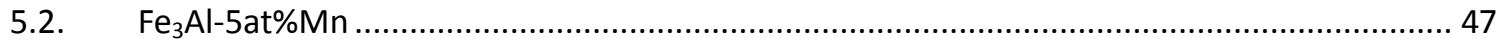

$\begin{array}{lll}\text { 5.2.1. } & \text { Analyse der AtomVicinity-Dichteprofile } & 47\end{array}$

5.2.2. Simulationen der Messungen 49 


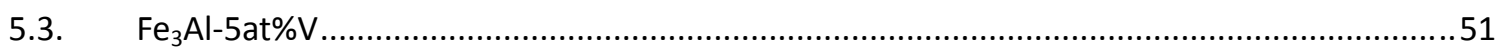

5.3.1. Analyse der AtomVicinity-Dichteprofile $\quad 51$

5.3.2. Simulationen der Messungen $\quad 53$

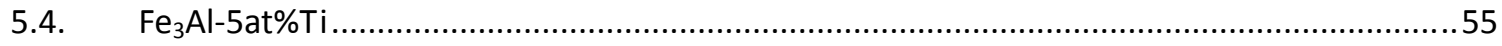

5.4.1. Analyse der AtomVicinity-Dichteprofile $\quad 55$

$\begin{array}{ll}\text { 5.4.2. Simulationen der Messungen } & 57\end{array}$

Kapitel 6 : ALCHEMI-Messungen an massivem Fe ${ }_{3}$ Al............................................................61

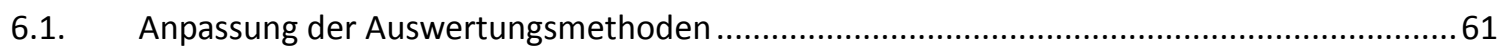

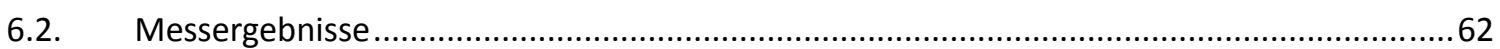

Kapitel 7 : Messungen an nanokristallinem ternären Fe $\mathrm{F}_{3} \mathrm{Al}$....................................................63

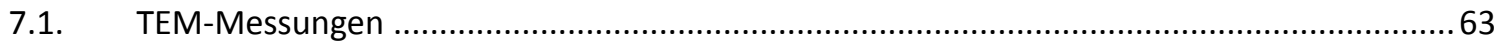

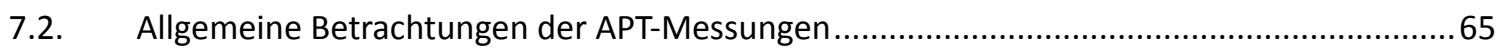

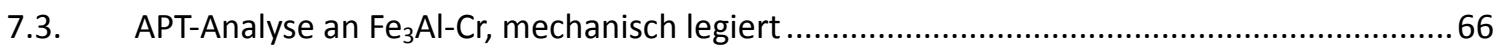

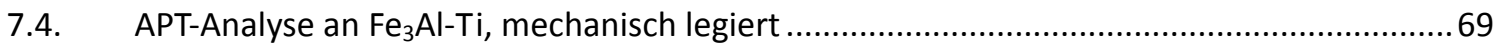

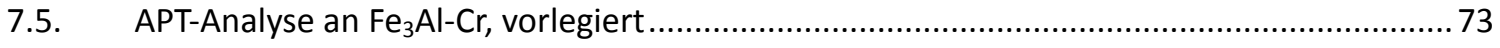

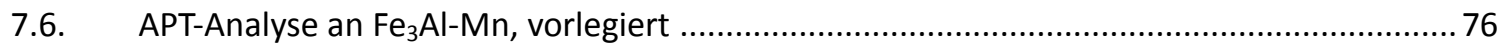

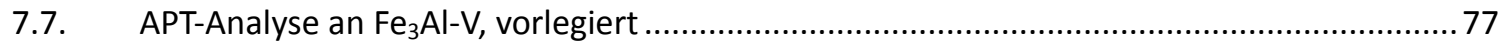

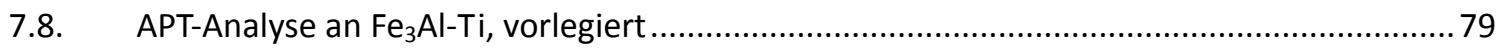

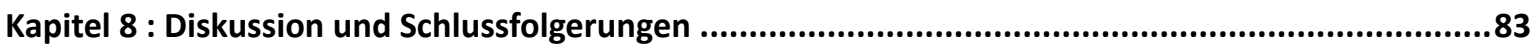

8.1. Qualitative Analyse von APT-Messungen an massiven, geordneten Proben ......................83

8.1.1. AtomVicinity-Dichteprofile 83

8.1.2. Evaporationsverhalten von $\mathrm{Fe}_{3} \mathrm{Al} \quad 84$

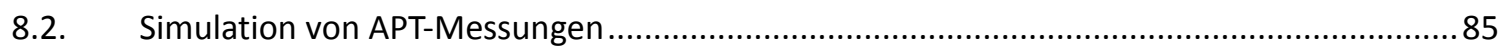

8.2.1. Allgemeine Betrachtung und Weiterentwicklungen 85

8.2.2. Mess- und Simulationsgenauigkeit 85

8.2.3. Paarpotentiale 86

$\begin{array}{ll}\text { 8.2.4. Simulationsparameter } & 87\end{array}$

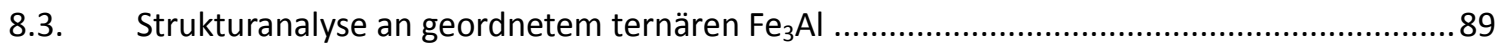

8.3.1. Allgemeine Analysen zur Platzbesetzung 89

$\begin{array}{ll}\text { 8.3.2. Ordnungsgrade } & 90\end{array}$

8.3.3. Zusammenhang mit mechanischen Eigenschaften 91

8.4. Allgemeine Betrachtung der Messungen an nanokristallinem ternären $\mathrm{Fe}_{3} \mathrm{Al}$...................99

8.4.1. Vorcharakterisierung durch XRD- und TEM-Messungen 92

8.4.2. Allgemeine Eigenschaften und Fortschritt der APT-Messungen 92

8.5. Gefügeanalyse von nanokristallinem ternären $\mathrm{Fe}_{3} \mathrm{Al}$.................................................99

$\begin{array}{ll}\text { 8.5.1. Verunreinigungen } & 94\end{array}$

8.5.2. Homogenität 95

8.5.3. Korrelierte Segregation 95

8.5.4. Gesamtbetrachtung des Gefüges 97 


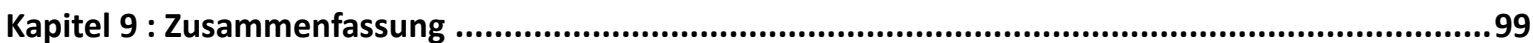

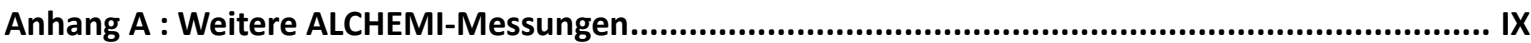

A.1. Theoretische Betrachtungen ....................................................................................... IX

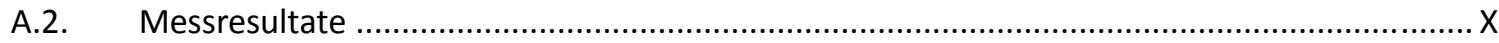

Anhang B : Messdaten und Simulationsparameter ........................................................................... XI

B.3. Materialparameter der Simulationen ............................................................................. XI

B.4. Messdaten der ALCHEMI (Zweistrahlmessverfahren) ............................................................ XI

Anhang C : Abschätzungen.............................................................................................................. XII

C.1. Einfluss der Projektion auf Atompositionen der APT-Simulation ........................................ XII

C.2. APT-Fehlrekonstruktion durch Unterschiede in Feldverdampfungsfeldstärken zweier

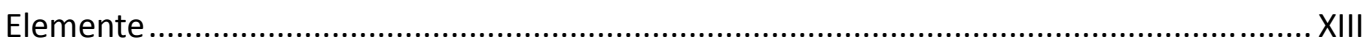

Literaturverzeichnis ..................................................................................................................... XIV

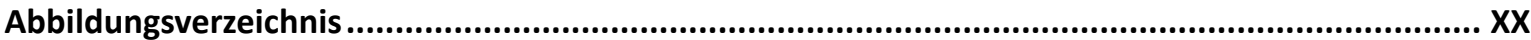

Akademischer Lebenslauf ........................................................................................................ XXIII

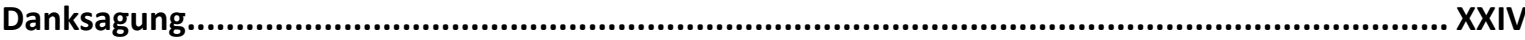




\section{Abkürzungen und Begriffe}

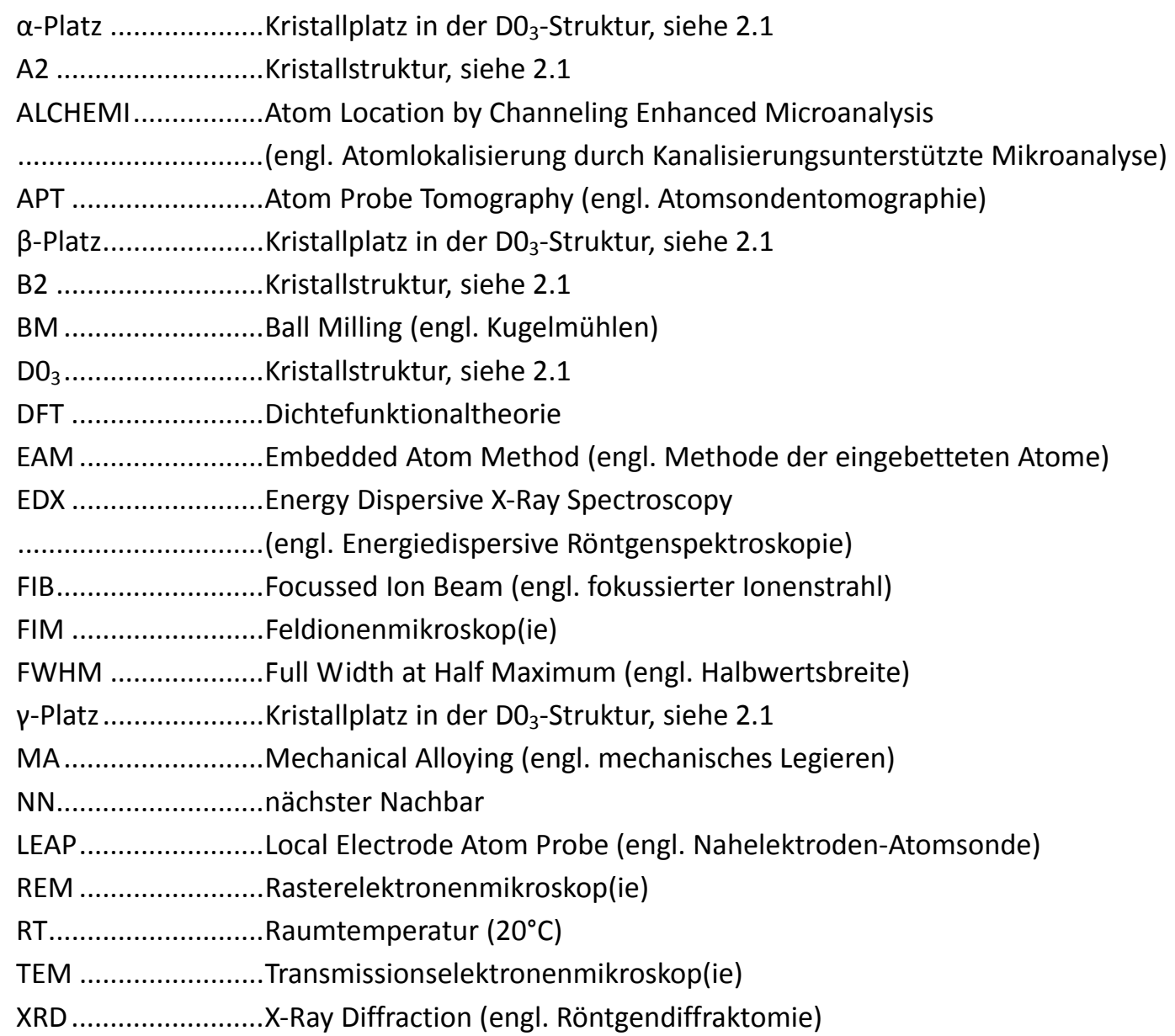




\section{Größen}

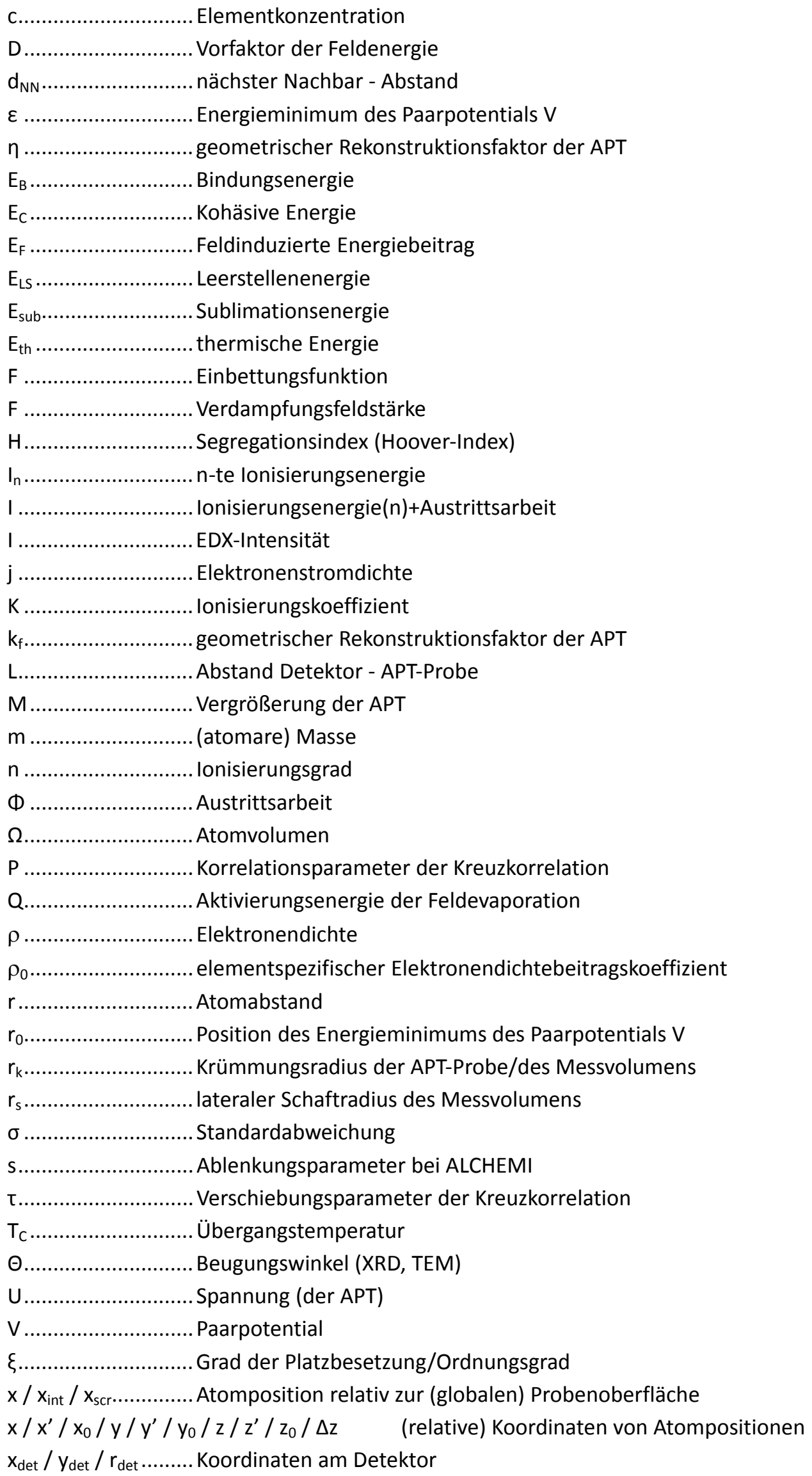




\section{Kapitel 1: Einleitung}

In der Materialwissenschaft ist es eine stete Herausforderung, die Struktur und Eigenschaften von Materialien zu charakterisieren und zu optimieren. Neue Erkenntnisse können heutzutage insbesondere durch die Untersuchung von immer kleineren Strukturen gewonnen werden. Dabei ist gerade auch die Analyse in atomaren Bereichen mit kontinuierlich weiter steigender Auflösung ein Teil moderner Forschung.

Dies ist der Grund, warum die genauere Erforschung von Eisenaluminiden gegenwärtig noch immer von Interesse ist, obwohl Vorzüge dieses Materials bereits in den 1930ern erkannt wurden. Schon damals sind die Vorteile der guten Korrosionsbeständigkeit, der geringen Dichte und der relativ niedrigen Herstellungskosten erkannt worden [1].

Weitergehende und umfassendere Studien der mechanischen Eigenschaften folgten in den 1950ern und 1960ern. Diese zeigten, dass Eisenaluminide, insbesondere $\mathrm{Fe}_{3} \mathrm{Al}$, eine hohe Festigkeit besitzen. Durch die gute Oxidationsbeständigkeit ist dieses Material geeignet, um z.B. in Heiz- und Ofenelementen Verwendung zu finden. Daneben sind auch Turbinen-, Motor- und Abgasanlagenteile, aber auch Anlagen der chemischen und nahrungsverarbeitenden Industrie sinnvolle Einsatzorte dieser Legierung [2].

Jedoch sind die Anwendungsbereiche bisher durch die geringe Duktilität bei Raumtemperatur und dem Festigkeitsabfall für Temperaturen über dem Phasenübergang bei ca. 820K eingeschränkt. Daher ist es von hohem Interesse, durch Addition ternärer Elemente und gezielter mikrostruktureller Behandlung die mechanischen Eigenschaften zu verbessern.

So ist für den Zusatz verschiedener Übergangsmetalle gezeigt worden, dass sie die Verformbarkeit erhöhen können, am stärksten ist dieser Effekt bei Chrom und Mangan ausgeprägt [2-4]. Auch die Phasenübergangstemperatur - und den damit verbundenen Festigkeitsabfall - kann damit zu höheren Temperaturen verschoben werden, wofür sich am besten Titan und Vanadium eignen $[5,6]$.

Ebenso ist eine stabilisierende Wirkung dieser Zusätze auf nanokristallines Gefüge gezeigt worden [4, 5 , 7]. Dieses weist eine gesteigerte Duktilität durch erhöhte Kriechraten und den Effekt der Superplastizität auf, was den Anwendungsbereich vergrößert $[1,8]$. Nanokristalline Eisenaluminide sind daher Thema aktueller Forschung.

Um die Eigenschaften von $\mathrm{Fe}_{3} \mathrm{Al}$ weiter zu verbessern, ist die genaue Kenntnis der atomaren Strukturen unabdingbar. Erst durch das präzise Wissen von Ordnungseinstellung, Homogenität und Segregation auf Nanometerskala können die genauen Effekte der Zusätze auf das mechanische Verhalten verstanden werden. Es bildet damit die Grundlage für eine weitere, gezielte Optimierung des Materials.

Die tomographische Atomsondentechnologie (APT) hat sich in den letzten Jahren rasant entwickelt. Mit ihrer Hilfe ist es heute möglich, bei einer Vielzahl von Materialien die atomare Struktur bis auf wenige Ångström genau zu charakterisieren. Dabei können Volumina mit Ausmaßen von einigen hundert Nanometern und Millionen von gemessenen Atomen erreicht werden.

In der vorliegenden Arbeit soll diese Technik zum ersten Mal verwendet werden, um die Ordnung von ternärem $\mathrm{Fe}_{3} \mathrm{Al}$ zu bestimmen. Bei der massiven, höchstmöglich geordneten Phase bilden die Bestimmung der Platzbesetzung der Zusätze, sowie die Messung der Ordnungsgrade der beteiligten Elemente den Schwerpunkt der Untersuchungen. Diese sind in Kapitel 4 und Kapitel 5 zu finden und werden - wie sich zeigen wird - sinnvoll durch den Einsatz von Simulationen von APT-Messungen unterstützt. Zu Vergleichszwecken durchgeführte ALCHEMI-Untersuchungen werden in Kapitel 6 vorgestellt. 
Des Weiteren werden auch erstmals APT-Messungen an nanokristallinem $\mathrm{Fe}_{3} \mathrm{Al}$ durchgeführt. Bei diesen Strukturen sind insbesondere Erkenntnisse der Homogenität auf atomarer Skala von hohem Interesse. Zusätzlich werden mögliche Segregation und Präzipitation sowie deren genaue Zusammensetzung und Ausmaße untersucht. Diese Studien, sowie ergänzende Untersuchungen mithilfe der Transmissionselektronenmikroskopie, sind in Kapitel 7 zu finden.

Zunächst sollen jedoch die theoretischen Hintergründe und der aktuelle Stand der Forschung in Kapitel 2 und Kapitel 3 genauer betrachtet werden. Abschliessend erfolgt eine Diskussion und Zusammenfassung der Erkenntnisse in Kapitel 8 und Kapitel 9. 


\section{Kapitel 2: Eisenaluminide}

Beginnend soll an dieser Stelle eine Übersicht über Eigenschaften von Eisenaluminiden gegeben und damit der aktuelle Stand der Forschung betrachtet werden. Bei massivem $\mathrm{Fe}_{3} \mathrm{Al}$ wird neben mechanischen und strukturellen Eigenheiten insbesondere die Platzbesetzung ternärer Elemente erörtert. Für nanokristalline Eisenaluminide sind Herstellungsprozesse und deren strukturelle Einflüsse von Interesse.

\subsection{Phasen und mechanische Eigenschaften}

Eisen und Aluminium sind gut ineinander löslich, wie das Phasendiagramm (Abbildung 2.1) verdeutlicht. Eisenaluminide liegen für Aluminiumkonzentrationen $c_{A l}$ bis etwa 50 at\% als raumzentrierte Kristallstruktur vor. Für höhere Aluminiumkonzentrationen sind etliche weitere Phasen zu finden, die flächenzentrierte oder komplexere Kristallstrukturen aufweisen [9].

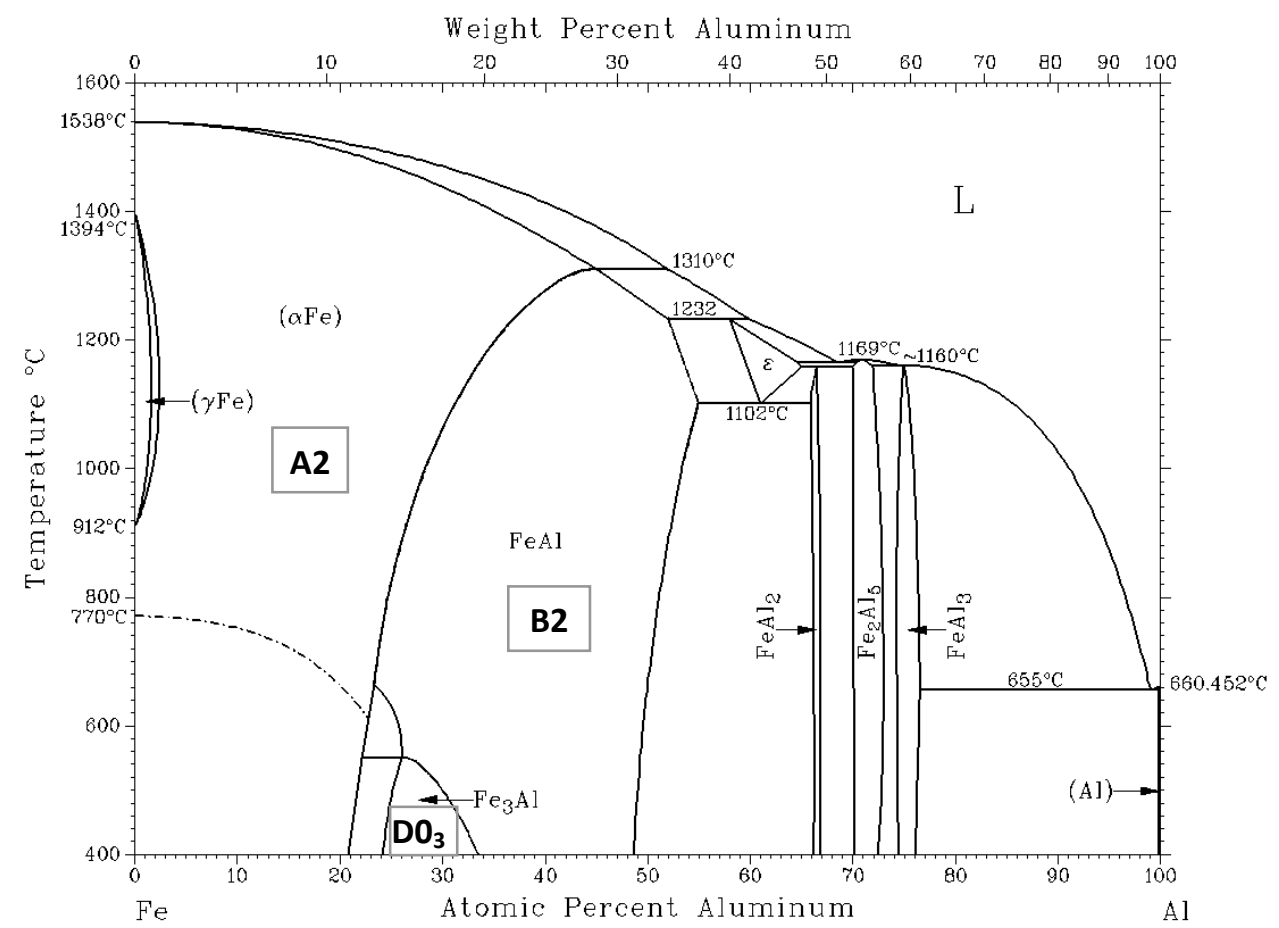

Abbildung 2.1: Phasendiagramm Eisen-Aluminium (nach MASSALSKI [10])

Für den erstgenannten Konzentrationsbereich sind im Wesentlichen drei Phasen zu finden: A2-, B2und $\mathrm{DO}_{3}$-geordnet. In der A2-geordneten Phase für Konzentrationen $\mathrm{c}_{\mathrm{Al}}$ unterhalb von etwa 20at\% (bei höheren Temperaturen bis zu 40at\%) sind die Aluminiumatome im Eisen statistisch substituell verteilt. Für jeweils höhere Aluminiumkonzentrationen $\mathrm{c}_{\mathrm{Al}}$ bis 50 at\% besetzt das Aluminium in der B2-Phase die raumzentrierten Plätze.

Für den Bereich der stöchiometrischen Konzentration von $\mathrm{Fe}_{3} \mathrm{Al}$ liegt unterhalb von ca. $820 \mathrm{~K}$ die $\mathrm{DO}_{3}$ Struktur vor. Es handelt sich dabei um eine flächenzentrierte Einheitszelle, die die drei Kristallplätze $\alpha$, $\beta$ und $\gamma$ aufweist (siehe Abbildung 2.2). Sie besteht aus 8 Oktanten, deren Eckatome mit Eisen besetzt 
sind ( $\alpha$-Plätze), und deren Zentralatome alternierend mit Eisen und Aluminium besetzt sind ( $\beta$ - bzw. $\gamma$ Plätze).

Unter den verschiedenen stöchiometrischen Phasen ist $\mathrm{Fe}_{3} \mathrm{Al}$ diejenige mit der höchsten Festigkeit [1]. Wie Abbildung 2.3 verdeutlicht, nimmt diese mit zunehmender Temperatur bis zu einem Minimum bei 650K ab. Danach steigt sie wieder bis zu einem ausgeprägten Maximum bei der $\mathrm{DO}_{3}$-B2-Phasenübergangstemperatur $\mathrm{T}_{\mathrm{C}}$; bei noch höheren Temperaturen fällt sie wieder deutlich ab. Diese sogenannte Fließgrenzenanomalie ist vielfach für verschiedene $\mathrm{Fe}_{3} \mathrm{Al}$-Basislegierungen gemessen worden [11, 12].

Ihre Ursache ist nicht genau erforscht, es gibt verschiedene Ansätze, diesen Effekt zu erklären: So wurde ein erhöhtes Quergleiten zwischen Gleitebenen kleinster PEIERLSNABARRO-Kraft in $\{110\}$-Richtung und $\{112\}$-Gleitebenen, die bei höheren Temperaturen auftreten, vorgeschlagen.

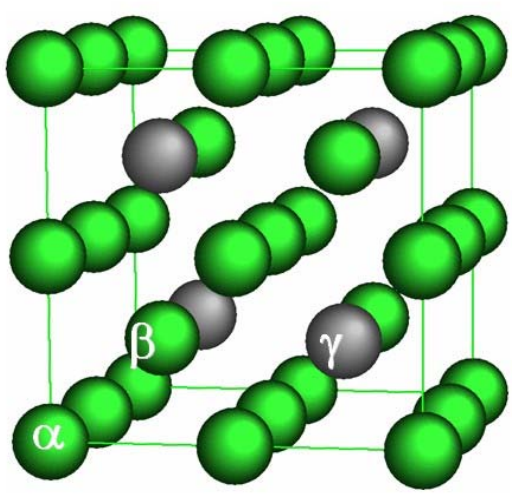

Abbildung 2.2: $\mathrm{Fe}_{3} \mathrm{Al}$ Einheitszelle in der $\mathrm{DO}_{3}-$ Struktur

Eisen, Aluminium Auch ein erhöhter Übergang von $<111>-$ zu $<110>-$ und $<100>$-Versetzungen wurde diskutiert. Daneben ist auch der Abbau von Antiphasengrenzen (und der folglich erschwerten Bewegung von <111>-Versetzungen) sowie Leerstellenhärtung genannt worden [1]. Genauere Studien lassen vermuten, dass mehrere Einflüsse zur Fließgrenzenanomalie beitragen und diese auch stark von der genauen Komposition und Gefügestruktur abhängen [2].

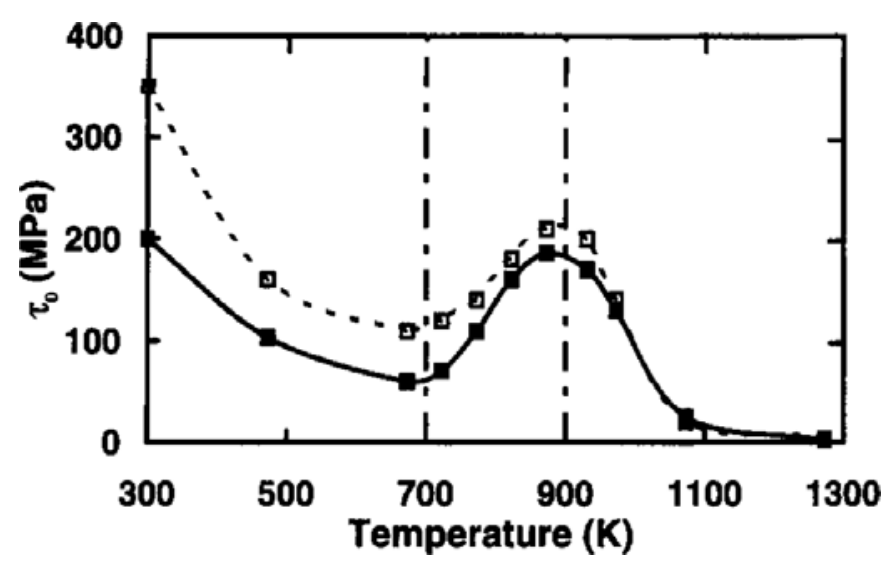

Abbildung 2.3: Temperaturabhängigkeit der kritischen Scherspannung

von $\square$ : Fe-28at\%Al a:Fe-28at\%Al-6at\%Cr (nach KRÁL et al. [11])
Als ternärer Zusatz ist insbesondere Chrom derjenige, welcher die Duktilität bei Raumtemperatur am stärksten erhöht [2, 3]. Dieser Effekt wurde auch bei 1,5at\% Mangan festgestellt [4]. Beide Zusätze führen jedoch auch zu einem Festigkeitsabfall (vgl. Abbildung 2.3).

Hingegen sind Vanadium und insbesondere Titan die effektivsten Additionen, um die Übergangstemperatur $T_{C}$ um bis zu $280 \mathrm{~K}$ zu erhöhen. Beide Elemente sorgen damit für eine erhöhte Warmfestigkeit, die Härte bei Raumtemperatur sinkt jedoch und außerdem ist eine ausgeprägte Verringerung der Duktilität zu verzeichnen. $[5,6,13,14]$

Insgesamt sind die Zusätze Chrom, Mangan, Vanadium und Titan diejenigen, die bestimmte mechanische Eigenschaften am deutlichsten verbessern. Dabei ist im Allgemeinen immer das gegensätzliche Verhalten bei der Optimierung von Festigkeit und Duktilität zu erkennen: (warm-) festere Legierungen zeigen spröderes Verhalten, duktilere Legierungen sind in der Regel weicher.

Warum diese Additionen derart unterschiedliche Veränderungen der Eigenschaften hervorrufen, ist nicht abschließend geklärt. Die erhöhte Duktilität durch Zugabe von Chrom kann durch das Absenken der Antiphasengrenzenenergie erklärt werden [1, 3], was die Verformung über die \{110\}-Gleitebene begünstigt. Für andere Gleitsysteme ist hingegen auch der gegenteilige Effekt gefunden worden, was 
aufgrund der oben genannten Eigenschaften zur Versetzungsbewegung zu einer deutlichen Temperaturabhängigkeit der Einflüsse durch Chromzugabe führt [11]. Ebenso bringt das auch eine ausgeprägte Abhängigkeit des genauen Ordnungsgrades, der Platzbesetzung und der Komposition mit sich.

Der Festigkeitsanstieg bei höheren Temperaturen durch Titan und Vanadium ist die Folge der Bildung von festeren Strukturen als LAVES- (-ähnliche) Phasen [15]. Hier macht sie die Atomgröße bei der Besetzung der $\beta$-Plätze bemerkbar [6]. Allerdings ist dennoch ein uneinheitlicher Einfluss auf den Gitterparameter der $\mathrm{DO}_{3}$-Struktur erkannt worden, dieser steigt bei Zugabe von Titan und etwas geringer auch bei Mangan, er sinkt hingegen bei Chrom und Vanadium [5, 13]. Daher ist anzunehmen, dass die genannten Unterschiede der makroskopischen Eigenschaften durch die genaue Struktur und den Ordnungsgrad nicht unentscheidend beeinflusst werden.

Auch Superplastizität wurde bei $\mathrm{Fe}_{3} \mathrm{Al}$ beobachtet, es wurden Dehnungen von 620\% ( $\mathrm{Fe}_{3} \mathrm{Al}-2 \mathrm{at} \% \mathrm{Ti}$ bei 1123K [1]) bzw. 350\% ( $\mathrm{Fe}_{3} \mathrm{Al}-3 \mathrm{at} \% \mathrm{Cr}$ bei 1073K [8]) gemessen. Bemerkenswert ist die Tatsache, dass der

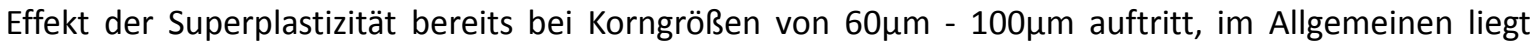
diese Grenze bei Legierungen bei ca. $1 \mu \mathrm{m}-5 \mu \mathrm{m}$. Diese Tatsache ist ein Grund für das hohe Interesse an der weiteren Erforschung von fein- und nanokristallinen $\mathrm{Fe}_{3} \mathrm{Al}$-Basislegierungen.

\subsection{Platzbesetzung und Ordnung ternärer Elemente}

Die Ordnung von ternärem $\mathrm{Fe}_{3} \mathrm{Al}$ wird in der Literatur häufig derart beschrieben, dass Elemente links des Eisens im Periodensystem - also alle in dieser Arbeit untersuchten Elemente - die $\beta$-Plätze in der $\mathrm{DO}_{3}$-Einheitszelle besetzen [5]. Bei genauerer Begutachtung entsprechender Studien stellt man jedoch fest, dass das Bild durchaus differenzierter ist und teilweise widersprüchliche Ergebnisse existieren:

So fanden NISHINO et al. [5] mithilfe von Röntgendiffraktomie (X-Ray Diffraction - XRD) heraus, dass Vanadium im Wesentlichen die $\beta$-Plätze besetzt, wohingegen POPIEL et al. [16] mit demselben Messverfahren zu dem Ergebnis einer $\alpha$-Platzbesetzung kam. Auch FORTNUM und MIKKOLA [17] untersuchten bei $400^{\circ} \mathrm{C}$ ausgelagertes $\mathrm{Fe}_{3} \mathrm{Al}-4$, 5 at\%Ti mithilfe von XRD. Unter der Annahme einer "vollkommenen Ordnung der $\mathrm{DO}_{3}$-Einheitszelle" [S.226] konnten sie für Titan eine $\beta$-Platzbesetzung finden.

Als eine weitere Methode zur Bestimmung der Platzbesetzung kann die Mössbauerspektroskopie eingesetzt werden. SATULA ermittelte so für $\mathrm{DO}_{3}$-geordnetes $\mathrm{Fe}_{3} \mathrm{Al}$ eine Bevorzugung der $\beta$-Plätze durch Chrom anhand der Platzbesetzungsgrade von Eisen [18]. Inkonsequent erscheint in dieser Studie, dass bis 5 at\% $\mathrm{Cr}$ die Fe-Konzentration nur auf $\alpha$-Plätzen sinkt, während sie auf $\beta$-Plätzen stabil bleibt und dort erst bei höheren $\mathrm{Cr}$-Konzentrationen abnimmt. In einer späteren Veröffentlichung, in der auch XRD-Messungen berücksichtigt wurden, erläutert SATULA, dass Chrom zusätzlich auch $\psi$-Plätze besetzen könne [19]. Auch ATHANASSIADIS benutzte Mössbauerspektroskopie um Proben zu untersuchen, die bei $950^{\circ} \mathrm{C}$ homogenisiert wurden und anschließend mit $4 \mathrm{~K} / \mathrm{h}$ abgekühlt wurden [20]. Er nahm an, dass "Titan und wahrscheinlich Mangan“ [sic, S.1] die $\beta$-Plätze besetzt, bei Mangan kann eine ebenfalls auftretende $\alpha$-Platzbesetzung nicht ausgeschlossen werden.

Mithilfe der ALCHEMI-Messmethode (siehe auch Kapitel 6) fanden YAN et al. eine $\gamma$-Platzbesetzung durch Cr-Atome in geordnetem $\mathrm{Fe}_{3} \mathrm{Al}$ [21].

KIM und MORRIS untersuchten mit Neutronendiffraktometrie $\mathrm{Fe}_{3} \mathrm{Al}-5$ at $\% \mathrm{Cr}$ ausgelagert bei $400^{\circ} \mathrm{C}$ und erhielten jedoch das Ergebnis einer $\beta$-Platzbesetzung durch $\mathrm{Cr}$ [22]. Auch ZUQING et al. erforschten Platzbesetzungen mit Neutronenstreuung an bei $500^{\circ} \mathrm{C}$ ausgelagertem $\mathrm{Fe}_{3} \mathrm{Al}$ [23]. $\mathrm{Cr}$ und Ti konnten als $\beta$-Platz besetzend erkannt werden, wohingegen $\mathrm{Mn}$ auf $\alpha$-Plätzen lokalisiert ist. MAGER et al. untersuchten die Platzbesetzung von Mangan mit Neutronen- und Röntgendiffraktometrie [24]. Das Ergebnis war, dass Mn-Atome die $\beta$ - und $\alpha$-Plätze im Verhältnis von 1:1 besetzen, unabhängig von der Gesamtmangankonzentration. Die verwendeten Proben wurden dabei bei $575^{\circ} \mathrm{C}$ ausgelagert, so dass 
gegebenenfalls die $\mathrm{DO}_{3}$-Struktur, welche sie mit XRD-Messungen nachwiesen, nicht mehr vollständig erhalten war.

Es gibt also durchaus widersprüchliche Ergebnisse in den genannten Studien. Als Ergänzung und zum Vergleich sollen hier auch Studien über die Platzbesetzungen in B2-geordneten Eisenaluminiden genannt werden:

So führte ANDERSON ALCHEMI Studien an B2-FeAl mit den Kompositionen $\mathrm{Fe}_{50} \mathrm{Al}_{45} \mathrm{Metall}_{5}$ bei $400^{\circ} \mathrm{C}$ [25] durch. $\mathrm{Cr}, \mathrm{V}, \mathrm{Ti}$ besetzen demnach die Al-Plätze, $\mathrm{Mn}$ im Wesentlichen die Fe-Plätze. Interessant ist hier der Ordnungsgrad der Aluminiumplatzbesetzung, der am höchsten für Titan mit $85 \%$ ist und für $V$ und $\mathrm{Cr}$ abnimmt, hingegen besetzt Mn Eisenplätze zu 70\%. Daraus ergibt sich, dass die Eisenplatzbesetzung dieser vier Zusätze desto geringer ist, je weiter das Element im Periodensystem vom Eisen entfernt ist. LAKSHMI entdeckte mithilfe der Mössbauerspektroskopie hingegen eine bevorzugte Eisenplatzbesetzung von Chrom in $\mathrm{B}^{2}-\mathrm{Fe}_{3} \mathrm{Al}[26]$.

Neben diesen experimentellen Studien gibt es auch zahlreiche theoretische Berechnungen:

Für $\mathrm{DO}_{3}$-geordnetes $\mathrm{Fe}_{3} \mathrm{Al}$ ermittelten REDDY et al. durch DFT-Berechnungen (Dichtefunktionaltheorie), dass aus energetischen Gründen $\mathrm{Cr}, \mathrm{Mn}, \mathrm{V}$ und $\mathrm{Ti}$ auf $\beta$-Plätzen sitzen müssen [27]. Bei $\mathrm{Cr}$ und $\mathrm{Mn}$ ist der energetische Aufwand zum Übergang auf einen $\alpha$-Platz dabei deutlich geringer als bei den anderen Elementen und liegt bei unter $0,7 \mathrm{eV}$. FUKS et al. berechneten mit DFT, dass Ti auf $\beta$-Plätzen, $V$ auf $\gamma^{-}$ Plätzen und $\mathrm{Mn}$ auf $\alpha$-Plätzen sitzen müssten, während es für Chrom keine energetische Bevorzugung unter den Eisenplätzen gebe [28].

FU und ZOU führten First-Principle-Berechnungen durch und bestätigten eine bevorzugte Aluminumplatzbesetzung durch Chrom und Titan in B2-FeAl [29]. Auch die ab-initio Berechnungen von MEDVEDEVA zeigen, dass in FeAl alle betrachteten Zusätze auf Aluminumplätzen sitzen [30]. CAl et al. errechneten mit der Thight-Binding-Methode, dass Ti, $\mathrm{Cr}$ und $\mathrm{V}$ auf $\alpha$-Plätzen sitzen müssten [31], hingegen kam Go zu dem Ergebnis, dass Chrom $\beta$-Plätze besetzt [32].

Eine zusammenfassende Übersicht der verschiedenen Studien und deren Ergebnisse zeigt Tabelle 2.1. Insgesamt kann an dieser Stelle festgehalten werden, dass für $\mathrm{Cr}, \mathrm{Mn}$ und $\mathrm{V}$ keine eindeutige Platzbesetzung zu verzeichnen ist. Auch der Vergleich zur ungeordneten B2-Struktur bietet keine eindeutigen Tendenzen. Teilweise können uneinheitliche Ergebnisse mit einer nicht extensiv durchgeführten Temperaturbehandlung erklärt werden. Auch die theoretischen Berechnungen, die bei $\mathrm{Cr}$ und $\mathrm{Mn}$ einen nur geringen Energieunterschied für $\beta$ - und $\alpha$-Platzbesetzung ergeben, können in diese Richtung deuten. Dennoch weisen bei Chrom die meisten Experimente auf eine $\beta$-Platzbesetzung hin. Bei Titan sind entsprechende Aussagen eindeutiger, hier weisen alle Experimente und fast alle theoretischen Berechnungen auf eine Besetzung des $\beta$-Platzes. 


\begin{tabular}{|c|c|c|c|c|c|}
\hline \multirow{2}{*}{\multicolumn{2}{|c|}{$\begin{array}{l}\text { Struktur \& } \\
\text { Kristallplatz }\end{array}$}} & \multicolumn{4}{|c|}{ ternärer Zusatz } \\
\hline & & Chrom & Mangan & Vanadium & Titan \\
\hline $\mathrm{DO}_{3}$ & $\alpha$ & $\begin{array}{l}\text { (Mössbauer) } \\
\text { (DFT) } \\
\text { tight-binding }\end{array}$ & $\begin{array}{c}\text { XRD } \\
\text { Neutronenstreuung } \\
\text { DFT }\end{array}$ & $\begin{array}{c}\text { XRD } \\
\text { tight-binding }\end{array}$ & tight-binding \\
\hline $\mathrm{DO}_{3}$ & $\beta$ & $\begin{array}{c}\text { XRD } \\
\text { Mössbauer } \\
\text { Neutronenstreuung } \\
\text { tight-binding } \\
\text { DFT }\end{array}$ & $\begin{array}{c}\text { XRD } \\
\text { Mössbauer } \\
\text { DFT }\end{array}$ & $\begin{array}{l}\text { XRD } \\
\text { DFT }\end{array}$ & $\begin{array}{c}\text { XRD } \\
\text { Mössbauer } \\
\text { Neutronenstreuung } \\
\text { DFT }\end{array}$ \\
\hline $\mathrm{DO}_{3}$ & v & $\begin{array}{c}\text { ALCHEMI } \\
\text { (XRD) }\end{array}$ & & DFT & \\
\hline B2 & $\alpha$ & Mössbauer & ALCHEMI & & \\
\hline B2 & $\beta / \nu$ & $\begin{array}{l}\text { ALCHEMI } \\
\text { ab-initio }\end{array}$ & ab-initio & $\begin{array}{l}\text { ALCHEMI } \\
\text { ab-initio }\end{array}$ & $\begin{array}{l}\text { ALCHEMI } \\
\text { ab-initio }\end{array}$ \\
\hline
\end{tabular}

Tabelle 2.1: Übersicht von Studien zur Platzbesetzung ternärer Elemente in geordnetem $\mathrm{Fe}_{3} \mathrm{Al}$

Es genaueres, detaillierteres Studium dieser Eigenschaften ist daher sinnvoll. Insbesondere gibt es nur wenige Angaben zu Ordnungsgraden. Deshalb sollen in dieser Arbeit ebendiese Aspekte mithilfe der Atomsondentechnologie untersucht werden, also mit einer Methode, die noch nie zuvor für die Bestimmung der Platzbesetzung in $\mathrm{Fe}_{3} \mathrm{Al}$ verwendet wurde.

\subsection{Herstellung und Eigenschaften nanokristallinen Gefüges}

In den letzten Jahren sind etliche Studien zu nanokristallinen Metallen und auch zu Eisenaluminiden durchgeführt worden. Die meisten davon befassen sich mit stöchiometrischem FeAl und als eine Standardmethode zu ihrer Herstellung erweist sich der Einsatz von Kugelmühlen (ball milling - BM).

So stellten BONETTI et al. auf diese Weise FeAl-Pulver durch mechanisches Legieren (mechanical alloying - MA) aus reinen Elementpulvern her [33]. Mit XRD untersuchten sie die vorhandenen Strukturen und die Korngröße. So zeigten sich nach 32h Mahldauer keine Peaks der reinen Komponenten mehr im XRD-Spektrum, die Korngröße konnten sie auf $5 \mathrm{~nm}$ bestimmen. Dabei wurden auch noch geringe Anteile an $\mathrm{Al}_{5} \mathrm{Fe}_{2}$ und $\mathrm{Fe}_{3} \mathrm{AlC}_{\mathrm{X}}$ gefunden. Der Kohlenstoff entstammt nach ihren Vermutungen dem während der Präparation verwendeten Ethanol, prinzipiell ist auch ein Abrieb von Stahlkugeln in der Mühle möglich. In einer weiteren Studie stellten BONETTI et al. $\mathrm{Fe}_{3} \mathrm{Al}$ her, hier lag der minimale Korndurchmesser bei $15 \mathrm{~nm}$, die XRD-Peaks der reinen Phasen verschwanden bereits nach ca. $2 \mathrm{~h}$ Mahldauer.

Ein anderes Herstellungsverfahren ist Hochdrucktorsion (high pressure torsion - HPT), bei der ein längliches Probenstück stark verformt (verdreht) wird und so eine nanokristalline Struktur entsteht [34]. PITHAWALLA et al. berichten von der Herstellung von FeAl-Pulvern durch Laser Vaporisation with Controlled Condentsation ( $L V C C$ ), also der kontrollierten Kondensation der Metalle aus der Dampfphase [35]. LA et al. [36] fertigten $\mathrm{Fe}_{3} \mathrm{Al}$ durch die aluminothermische Reaktion $3 \mathrm{Fe}_{2} \mathrm{O}_{3}+8 \mathrm{Al} \rightarrow 2 \mathrm{Fe}_{3} \mathrm{Al}-3 \mathrm{Al}_{2} \mathrm{O}_{3}$ an, die bei $260^{\circ} \mathrm{C}$ einsetzt. Das Aluminiumoxid scheidet sich dabei im Tiegel oberhalb des $\mathrm{Fe}_{3} \mathrm{Al}$ ab. Durch XRD konnten sie Korngrößen von $10 \mathrm{~nm}-25 \mathrm{~nm}$ und eine ausgeprägte $\mathrm{DO}_{3}$-Struktur ermitteln.

SARKAR und BANSEL untersuchten die Ordnung in nanokristallinem $\mathrm{Fe}_{3} \mathrm{Al}$ mit XRD und Mössbauerspektroskopie [37]. Das durch Kugelmühlen hergestellte Pulver lagerten sie aus. Nach etwa 30h bei 
$300^{\circ} \mathrm{C}$ sinkt die Anzahl der Fe-Atome mit 2 - 3 Al-Atomen als nächster Nachbar (NN), die Anzahl mit 0 oder 4 Al-Atomen als NN steigt. Dies kann als Umordnung von einer $\mathrm{A} 2$ - in eine $\mathrm{DO}_{3}$-Struktur interpretiert werden, wobei letztere nicht vollkommen ausgeprägt zu sein scheint und andere Phasen daneben existieren. Die Korngrößen steigen dabei selbst bei $800 \mathrm{~h}$ Auslagerung bei $450^{\circ} \mathrm{C}$ nur geringfügig von $10 \mathrm{~nm}$ auf etwa $14 \mathrm{~nm}$ an.

Die einzige FIM-/1D-Atomsonden-Studie zu nanokristallinen Eisenaluminiden ist die von MELMED et al. über Fe-5,1at\%Al [38]. Sie benutzten zur Legierung aus reinen Elementen eine mit flüssigem Stickstoff gefüllte Kugelmühle, anschließend wurde das Material $1 \mathrm{~h}$ bei $900^{\circ} \mathrm{C}$ unter Argonatmosphäre konsolidiert. Dabei konnten Bereiche erhöhten Aluminiumgehalts an Korngrenzen aber auch in Körnern gefunden werden, meist verbunden mit Sauerstoff und Stickstoff. Des Weiteren konnten generell Aluminium, Nickel und Sauerstoff segregiert an Korngrenzen ausgemacht werden.

Studien über nanokristalline Eisenaluminide mit einem ternären Zusatz der in 2.2 betrachteten Elemente sind jedoch nur wenige zu finden. So berichten RAFIEl et al. von $\mathrm{Fe}_{3} \mathrm{Al}$ und $\mathrm{Fe}_{2} \mathrm{AlTi}$ [7]. Sie benutzen ebenfalls BM, wobei sie Homogenität erst nach 100h Mahldauer erreichten, gemessen mit XRD. Sie konnten zeigen, dass der Zusatz Titan die Korngröße verringert (von $35 \mathrm{~nm}$ auf $12 \mathrm{~nm}$ ) und eine höhere Löslichkeit aufzeigt, wodurch kürzere Mahldauern möglich sind. Titan erhöht außerdem deutlich die Festigkeit. Auch die $\mathrm{DO}_{3}$-Ordnung wird erhöht, insbesondere nach einer Auslagerung von einer Stunde bei $550^{\circ} \mathrm{C}$ ist die $\mathrm{DO}_{3}$-Struktur deutlich zu erkennen.

$\mathrm{LA}$ et al. untersuchten nanokristallines $\mathrm{Fe}_{3} \mathrm{Al}-\mathrm{Mn}$, welches mit der oben genannten aluthermischen Reaktion hergestellt wurde und 5at\% - 15at\% Mangan enthielt [39]. Sie stellten fest, dass in diesem Konzentrationsbereich durch das Mangan sowohl Korngröße als auch Festigkeit zunächst ansteigen, ab 10 at\% jedoch wieder geringfügig absinken. Eine $\mathrm{DO}_{3}$-Struktur konnte nur bei 15 at\% $\mathrm{Mn}$ ermittelt werden.

Die wichtigsten Strukturparameter aus den genannten Studien sind in Tabelle 2.2 noch einmal zusammengefasst, ergänzt durch einige weitere Arbeiten.

Zusammenfassend kann man festhalten, dass in FeAl bzw. Fe $\mathrm{A}_{3} \mathrm{Al}$ Korngrößen von wenigen Nanometern erreicht werden können. Außerdem zeigt sich, dass durch mechanisches Legieren homogene EisenAluminium-Phasen erzeugt werden können. Dabei kommt es zu deutlich schwankenden Werten der Mahldauer, die bis zur Homogenität nötig sind. Allerdings ist zu beachten, dass andere Faktoren (Bauart und Geometrie der Mühle, Drehzahlen, etc.) relevant sind, daher ist ein Vergleich dieser Werte kaum möglich. Stark herstellungsabhängig sind ebenfalls strukturelle Eigenheiten des Materials. Von allen drei möglichen Ordnungen von $\mathrm{Fe}_{3} \mathrm{Al}$ wurde berichtet. Dabei können ungeordnete Strukturen durch Anlassen bei geringeren Temperaturen oder Konsolidieren höhere Ordnungsgrade erreichen, ohne dass dabei das nanokristalline Gefüge wesentlich verloren geht. Weitere Phasen, insbesondere Carbide oder Oxide, können auftreten. Von herstellungsbedingten Verunreinigungen und damit verbundenen Folgen wie Segregation oder Entmischung ist wenig berichtet worden.

Gerade für letztgenannte Eigenschaften hat es allen Anschein, dass diese Phänomenologie systematisch nur mit der Atomsonde gemessen werden kann. Insbesondere die Bestimmung auch kleinerer (Fremd-) Elementkonzentrationen kombiniert mit der hohen Auflösung kann dabei zu neuen Erkenntnissen führen. 


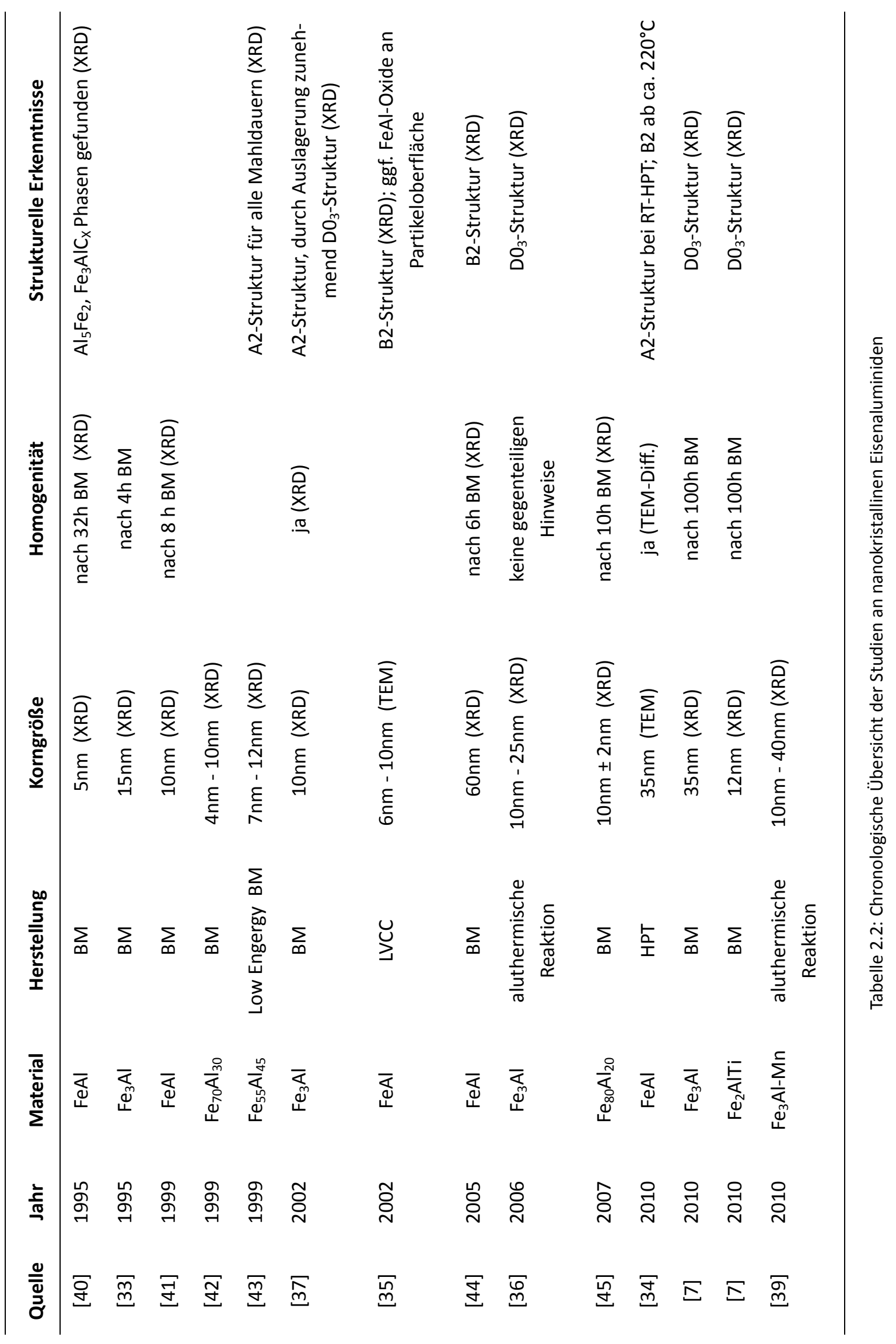




\section{Kapitel 3: Experimentelle Grundlagen}

An dieser Stelle soll ein Überblick über die Untersuchungsmethoden APT, FIM und ALCHEMI gegeben werden, sowie die Herstellung und Präparation der Proben. Insbesondere sollen die Prinzipien der APT dahingehend erläutert werden, die Arbeitsweise der APT-Simulation detailliert darlegen zu können. Des Weiteren sollen die Optimierungen und Neuerungen dieses Simulationsalgorithmus im Rahmen dieser Arbeit erklärt werden.

\subsection{Atomsondentomographie und Feldionenmikroskopie}

\subsubsection{Aufbau und Arbeitsprinzip}

Der prinzipielle Aufbau der Atomsondentomographie (atom probe tomography - APT) mit Feldionenmikroskop (field ion microscope - FIM) ist in Abbildung 3.1 schematisch dargestellt. Die zu untersuchenden Materialien liegen als Spitzen vor, die einen Krümmungsradius $r_{k}$ im vorderen Bereich der Probe von unter 70nm und einen dünnen Schaft mit einem Winkel von unter $18^{\circ}$ aufweisen. Die gesamte Anlage ist hochevakuiert.

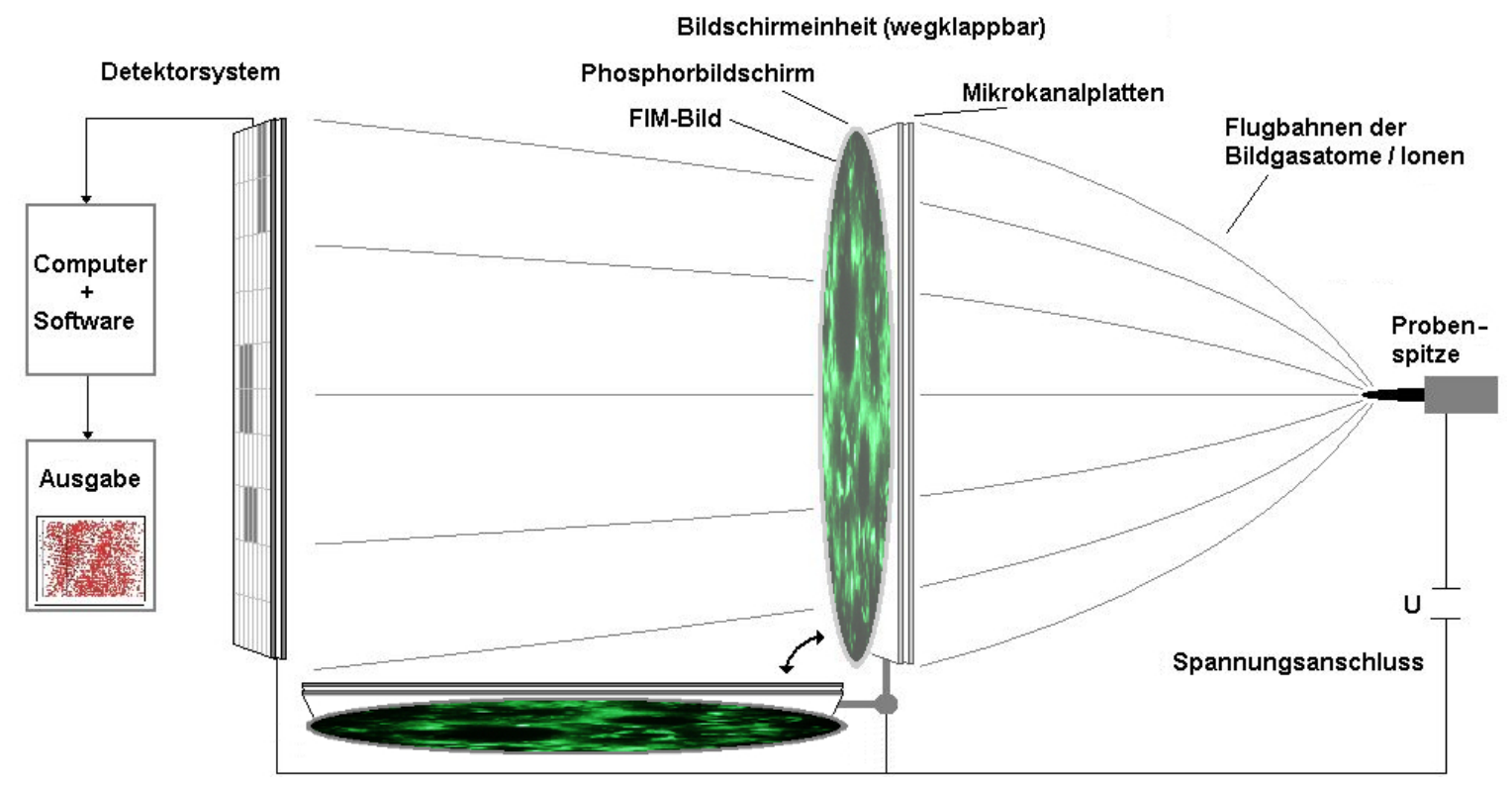

Abbildung 3.1: Schematischer Aufbau und Funktionsweise von FIM- und APT-Messungen

Zwischen der Probe und den etwa 45cm entfernten Mikrokanalplatten, die sich vor einem Multianodendetektor befinden, wird eine Hochspannung angelegt. Zusätzlich wird eine gepulste Spannung überlagert, die für Intervalle von ca. 10ns die Hochspannung um 10\% - 25\% erhöht. Durch das elektrische Feld können einzelne Oberflächenatome der Probe ionisiert und in Richtung Detektor beschleunigt werden, man spricht von Feldverdampfung. Anstatt durch eine überlagerte Spannung kann diese Anregung auch durch kurze Laserpulse erfolgen. Die Ionen erzeugen in den Kanalplatten eine Ladungswolke, die dann auf die Anoden des Detektors trifft. Durch den Ladungsschwerpunkt wird die 
laterale Position des Atoms an der Spitze bestimmt, durch die Abfolge die longitudinale Position. Anhand der gepulsten Spannungs- bzw. Laseranregung wird ein wohldefinierter Startpunkt des Ablösens des Atoms ausgemacht, durch das Detektorsignal kann so eine Flugzeitmessung durchgeführt werden. Aus dieser erfolgt nach dem Prinzip der Energieerhaltung eine Massenberechnung. Der Computer nimmt so einen Datensatz aus $x-, y$ - und $z$-Koordinaten eines jeden Atoms sowie seiner Masse auf, der dann zur weiteren Analyse zur Verfügung steht. Die Pulsspannung bzw. die Laserenergie ist so zu wählen, dass die Wahrscheinlichkeit der lonisation eines Atoms pro Puls bei etwa $1 \%$ liegt [46, 47].

Im FIM-Betrieb der Atomsonde wird im Abstand von ca. $5 \mathrm{~cm}$ vor der Spitze ein Phosphorbildschirm mit davor gelagerten Mikrokanalplatten positioniert. Zwischen diesen und der Sptize liegt eine konstante Hochspannung von bis zu ca. $20 \mathrm{kV}$ an. In das System wird ein nicht reaktives Bildgas eingelassen, für die vorliegenden Messungen erwies sich Neon mit einem Druck von $5 \cdot 10^{-5} \mathrm{~Pa}$ als ideal. Das Bildgas wird durch das elektrische Feld polarisiert und durch Kontakt zu der auf 20K - 80K gekühlten Probe thermalisiert. Im Bereich der Bestbildfeldstärke (Feldstärke des besten Bildkontrastes) werden die Gasmoleküle in Gebieten direkt an den Oberflächenatomen ionisiert, sodass sie im elektrischen Feld zu den Mikrokanalplatten beschleunigt werden. Die dadurch innerhalb der Kanalplatten erzeugten Elektronenkaskaden produzieren ein Bild auf dem Phosphorschirm mit einer Vergrößerung von etwa $10^{6}$. Dadurch werden Oberflächenatome als Leuchtpunkte innerhalb der FIM-Bilder ausgemacht. Eine Unterscheidung nach Elementen ist nicht direkt möglich, allerdings sind deren Helligkeiten ggf. aufgrund verschiedener Feldverdampfungsfeldstärken unterschiedlich.

Für eine ausführliche Beschreibung der Funktionsweise von FIM und APT sei auf Literatur verwiesen $[46,48]$. An dieser Stelle soll nur ein kurzer Abriss über die geometrischen Verhältnisse der Vergrößerung und Rekonstruktion der Atompositionen gegeben werden.

Das elektrische Feld $\mathrm{F}$ an der Probenoberfläche bei angelegter Spannung $\mathrm{U}$ hängt $\mathrm{u}$. a. von dem Krümmungsradius $r_{k} a b$ :

$$
F=\frac{U}{k_{f} \cdot r_{k}}
$$

Formel 3.1

Wobei $k_{f}$ ein Geometriefaktor ist, der zwischen 3 und 8 liegt. Diese Beschreibung gilt idealerweise für die gesamte Probenspitze. Zur genauen Bestimmung des Vergrößerungsfaktors $M$ kann man davon ausgehen, dass das Bild als Zentralprojektion auf dem Bildschirm abgebildet wird. Da die Trajektorien der lonen jedoch nicht geradlinig sind, muss ein Faktor $\eta$ eingeführt werden, dieser liegt etwa bei 1,5 [48]. Mit I $:=r_{k} \cdot \eta$ und dem Abstand L zwischen Probe und Schirm gilt laut Strahlensatz (vgl. Abbildung 3.2):

$$
M=\frac{L}{l}=\frac{L}{\eta \cdot r_{k}}=\frac{L \cdot F \cdot k_{f}}{\eta \cdot U}
$$

Formel 3.2

Die Berechnung der Atompositionen $\mathrm{x}, \mathrm{y}, \mathrm{z}$ bedarf also neben der Kenntnis der Position des Detektorsignals $\left(\mathrm{x}_{\mathrm{det}}, \mathrm{y}_{\mathrm{det}}\right)$, auch die der Feldverdampfungsfeldstärke des Probenmaterials, des Abstandes $\mathrm{L}$ und der Spannung $U$, welche zum Zeitpunkt der jeweiligen Evaporation anlag. Die Faktoren $k_{f}$ und $\eta$ müssen empirisch angepasst werden. Sinnvolle Anhaltspunkte für richtige Werte sind die rekonstruierten Kristallebenen, die eben und im richtigen Abstand zueinander liegen müssen. Die Berechnung der Koordinaten $x, y, z$ erfolgt näherungsweise, indem die Projektion quasi zurückverfolgt wird (siehe Abbildung 3.2). 


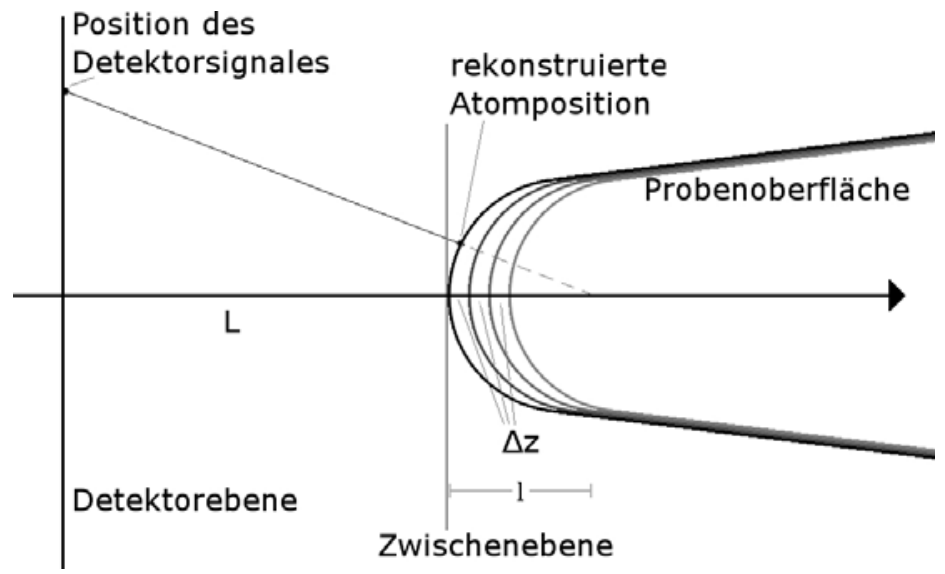

Abbildung 3.2: Geometrische Verhältnisse der Rekonstruktion von Atompositionen bei der APT

$$
\mathrm{x}=\frac{\mathrm{x}_{\mathrm{det}}}{\mathrm{M}} \quad \mathrm{y}=\frac{\mathrm{y}_{\mathrm{det}}}{\mathrm{M}} \quad \mathrm{z}=\sum \Delta \mathrm{z}+\mathrm{r}_{\mathrm{k}}-\sqrt{\mathrm{r}_{\mathrm{k}}^{2}-\left(\frac{\mathrm{x}_{\mathrm{det}}}{\mathrm{M}}\right)^{2}-\left(\frac{\mathrm{y}_{\mathrm{det}}}{\mathrm{M}}\right)^{2}}
$$

Dabei wird von einer Halbkugel als (vordere) Probenform ausgegangen. Wichtig ist dabei der Aspekt, dass die Probe mit jedem evaporierten Atom kürzer wird, d.h. dass die Probenoberfläche und die Zwischenebene in Abbildung 3.2 jeweils um $\Delta z$ nach rechts verschoben wird. $\Delta z$ ist dadurch geeicht, dass in dem rekonstruierten Volumen die Atomdichte der der Probe entspricht, in der Abbildung ist $\Delta z$ also stark vergrößert dargestellt [49].

\subsubsection{Auswertungsmethoden der Atomsondentomographie}

\section{AtomVicinity-Algorithmus}

Wie Abbildung 4.3 verdeutlicht, weisen die gemessenen APT-Volumina in Messrichtung (z-Richtung) die höchste Auflösung auf [46]. So lassen sich prinzipiell Dichteprofile in dieser Richtung anfertigen, die mit den theoretischen Dichteprofilen verglichen werden können, um Ordnung und Platzbesetzung zu bestimmen [50, 51]. Bei geringen Konzentrationen sind die statistischen Schwankungen - resultierend aus Messungenauigkeiten und der statistischen Verteilung - aber ggf. zu groß für eindeutige Interpretationen.

Um die Signifikanz zu erhöhen, wurde der AtomVicinity-Algorithmus entwickelt. Seine Arbeitsweise ist in [52] ausführlich beschrieben, sie soll an dieser Stelle kurz zusammengefasst werden: Ziel der Analyse ist es, die durchschnittliche Umgebung von bestimmten Atomen (z.B. die eines spezifischen Elements) zu ermitteln. Von diesen Atomen werden alle Umgebungen, d.h. alle Umgebungsatome innerhalb eines würfelförmigen Volumens definierter Kantenlänge, bestimmt. Sie werden dann über- bzw. ineinander gelegt, so dass das Resultat die summierten Umgebungen der bestimmten Zentralatome darstellt. In dieser Arbeit werden die Aluminiumatome als Zentralatom (also als Referenzatom) verwendet. Das ist sinnvoll, da Eisen zwei verschiedene Kristallplätze besetzt.

Ein Atom kann im Resultat mehrfach vorkommen, da es in mehreren Umgebungen anderer Atome liegt oder auch selbst ein zentrales Atom darstellen kann. Die positionsabhängige Häufigkeit von Atomen hat damit keine Aussage in absoluten Werten, allein die relative Häufigkeit ist von Relevanz. Diese freie Skalierung erlaubt es auch, von „summierter Umgebung“ oder „durchschnittlicher Umgebung“ der bestimmten Atome zu sprechen. Skaliert man die Häufigkeiten der Zentralatome auf 1, kann man ebenso von der „Antreffwahrscheinlichkeit“ eines Atoms relativ zum Zentralatom sprechen. 
Diese Analysemethode bietet durch die Summation eine höhere Signifikanz. Ein weiterer Vorteil dieses Algorithmus ist der resultierende Ausgleich von Rekonstruktionsfehlern. Zeigt das gesamte Volumen beispielweise eine Streckung oder eine Krümmung, kann dies Dichteprofile verwischen. Da im AtomVicinity-Algorithmus nur die relativen Abstände betrachtet werden, werden die globalen Fehlpositionierungen weitestgehend ausgelassen.

\section{Konzentrationsanalyse}

Neben der visuellen Darstellung der Messergebnisse der APT sind für quantitative Untersuchungen Konzentrationsanalysen von hoher Aussagekraft. Dazu wird das Volumen in Teile gleicher Größe oder gleicher Atomanzahl aufgeteilt und deren Konzentration der betrachteten Elemente ermittelt. So können z. B. eindimensionale Konzentrationsprofile erstellt werden. Die Konzentrationenverteilung folgt im homogenen Fall einer Binomialverteilung mit der Standardabweichung

$$
\sigma=\sqrt{\frac{\overline{\mathrm{c}} \cdot(1-\overline{\mathrm{c}})}{\mathrm{N}}}
$$

$\overline{\mathrm{C}}$ ist die mittlere Konzentration und $\mathrm{N}$ die Atomanzahl pro Block. Es liegen dann $68 \%$ aller Messpunkte im Bereich [ $\overline{\mathrm{C}}-\sigma, \overline{\mathrm{C}}+\sigma]$ und $95 \%$ im Bereich [ $\overline{\mathrm{C}}-2 \sigma, \overline{\mathrm{C}}+2 \sigma][53,54]$.

\section{Ungleichverteilungsmaß/Segregationsindex}

Um die Homogenität quantitativ genauer zu erfassen, kann für die Verteilung eines Elements ein Ungleichverteilungsmaß bzw. Segregationsindex berechnet werden. Dafür bietet sich der Hooverindex an, er beschreibt die mittlere relative Abweichung vom Mittelwert. Er stellt den direktesten Index dar, da er die Abweichung ohne eine zusätzliche Gewichtung mit einkalkuliert (im Gegensatz z.B. zum Ginioder Theil-Index) $[55,56]$.

Der Hooverindex betrachtet den relativen Anteil eines Elements A an einem Messpunkt im Vergleich zum relativen Anteil der gesamten Atome G. Die Hälfte der aufsummierten absoluten Differenzen ergibt den Hooverindex:

$$
H_{A}=\frac{1}{2} \sum_{i}^{n}\left|\frac{A_{i}}{A_{\text {ges }}}-\frac{G_{i}}{G_{\text {ges }}}\right|
$$

Formel 3.5

$A_{i}$ und $G_{i}$ sind die entsprechenden Atomanzahlen am Messpunkt $i$ und $A_{\text {ges }}$ bzw. $G_{\text {ges }}$ die jeweiligen Gesamtatomanzahlen. Sein Wert liegt damit zwischen 0 und 1 und beschreibt damit den Anteil der Atome, die zu einer vollkommenen Gleichverteilung verlagert werden müssten.

Der Segregationsindex $\mathrm{H}_{\text {hom }}$ im Falle einer statistischen, also homogenen, Atomverteilung wird dann berechnet, indem alle A-Atome, die über der mittleren Atomanzahl $\bar{A}$ vorliegen, aufsummiert und ins Verhältnis zu $\bar{A}$ gesetzt werden. Um diese Atomanzahl zu ermitteln, muss die Normalverteilung ${ }^{i}$ multipliziert mit der Atomanzahl ab $\bar{A}$ integriert werden.

${ }^{i}$ Die Binomialverteilung wurde hier durch die Normalverteilung genähert, was nach dem Satz von MoivreLaplace gerechtfertigt ist [54]. 


$$
\begin{aligned}
H_{A, h o m} & =\frac{1}{\bar{A}} \int_{\frac{A}{\sigma}}^{\infty} \frac{1}{\sigma \sqrt{2 \cdot \pi}} \cdot \exp \left(-\frac{1}{2} \cdot\left(\frac{A-\bar{A}}{\sigma}\right)^{2}\right) \cdot(A-\bar{A}) d A \\
& =\frac{1}{\bar{A}}\left[\frac{-\sigma}{\sqrt{2 \cdot \pi}} \cdot \exp \left(-\frac{1}{2} \cdot\left(\frac{A-\bar{A}}{\sigma}\right)^{2}\right)\right]_{\bar{A}}^{\infty}=\frac{\sigma}{\bar{A} \cdot \sqrt{2 \cdot \pi}}
\end{aligned}
$$

Wo $\sigma$ die Standardabweichung der A-Atomverteilung ist, die analog zu Formel 3.4 berechnet wird.

\section{Kreuzkorrelation/Verteilungsanalyse}

Um die Verteilung von verschiedenen Elementen zu vergleichen und eine mögliche Korrelation in den Konzentrationsprofilen zu untersuchen, ist die Kreuzkorrelation eine dienliche Auswertungsmethode. Dabei wird das Integral des Produktes zweier Verteilungsfunktionen in Abhängigkeit von einer relativen Verschiebung $\tau$ dieser beiden Funktionen bestimmt [57].

Für die Konzentrationsbestimmung mit diskreten Messpunkten muss das Integral dann durch eine Summe ersetzt werden, damit ergibt sich für den Korrelationsparameter $\mathrm{P}_{\mathrm{A}, \mathrm{B}}$ zwischen den Elementen A und $B$ :

$$
P_{A, B}(\tau)=\frac{1}{\left(x_{\max }-x_{\text {min }}-|\tau|\right)} \cdot \sum_{i} c_{A}\left(x_{i}\right) \cdot c_{B}\left(x_{i}+\tau\right)
$$

Dabei ist $\mathrm{i}$ der Index der Messpunkte und $\mathrm{x}_{\max }$ und $\mathrm{x}_{\min }$ der größte bzw. kleinste Messpunkt. Der Faktor vor der Summe dient der Skalierung auf die Anzahl betrachteter Messpunkte. Zusätzlich wird zum besseren Vergleich $P(\tau)$ derart normiert, dass $P(0):=1$ gilt.

Liegen die Elemente $A$ und $B$ im betrachteten Volumen segregiert vor, d.h. ist der Verlauf der Konzentrationen tendenziell gegensätzlich, so steigt $P_{A, B}$ für ein von Null verschiedenes $\tau$. Wenn $A$ und $B$ hingegen eine Überlagerung zeigen, da sie z.B. Ausscheidungen einer gemeinsamen Phase bilden, so gleichen sich die Konzentrationsverläufe in einem gewissen Rahmen und $\mathrm{P}_{A, B}$ findet ein Maximum bei $\tau=0$.

Anzumerken ist, dass die Kreuzkorrelation nicht nur eindimensional, sondern ebenso dreidimensional gilt, d.h. dass in Formel $3.7 \mathrm{x}$ und $\tau$ als Raumvektoren zu betrachten sind. In dieser Arbeit wird die Kreuzkorrelation stets dreidimensional durchgeführt, dadurch ergibt sich jedoch die Schwierigkeit, die Ergebnisse grafisch darzustellen. Diese wurde durch die Darstellung $P(|\vec{\tau}|)$ als zweidimensionaler Funktionsgraph behoben. Da unterschiedliche Vektoren $\vec{\tau}$ den gleichen Betrag haben können, kann es für ein $\tau:=|\vec{\tau}|$ also mehrere P-Werte geben. Die gewählte $P(\tau)$-Darstellung stellt folglich keine eindeutige, mathematische Funktion dar, ist jedoch übersichtlicher.

Diese Analyse kann ebenfalls für nur ein Element durchgeführt werden, das dann mit sich selbst verglichen wird. Diese Autokorrelationsanalyse hat per Definition bei $\tau=0$ ein Maximum und fällt danach für realistische Verteilungen ab. Eine Aussage über Segregation anhand des Verlaufes kann dann nur geschehen, wenn dieser mit dem Verlauf einer homogenen Verteilung verglichen wird. Da diese Betrachtung aber keinen Vorteil zur Berechnung des Segregationsindexes bietet, wird sie in dieser Arbeit nicht verwendet. 


\subsection{Probenpräparation}

\subsubsection{Geordnete, massive Materialen}

Das Probenmaterial ist am Max-Planck-Institut für Eisenforschung GmbH (MPIE) in Düsseldorf angefertigt worden. Dazu wurden fünf Legierungen aus reinen Metallen mit jeweils mindestens 99,99\% Reinheit eingeschmolzen: ${ }^{\text {ii }}$

- 74 at\% Fe, 26at\% Al

- 69at\% Fe, 26at\% Al, 5at\%Cr

- $69 a t \%$ Fe, $26 a t \%$ Al, 5at\%Mn

- 70 at\% Fe, 26at\% Al, 4at\%V

- 70 at\% Fe, 26at\% Al, 4at\%Ti

Nach dem Einschmelzen folgten eine 24-stündige Homogenisierung bei $1100^{\circ} \mathrm{C}$ und eine vierwöchige Auslagerung bei $450^{\circ} \mathrm{C}$, um eine optimale Ordnung und eine minimale Defektdichte zu erzielen.

Die weitere Probenpräparation und deren Messungen erfolgten am Institut für Materialphysik (IMP) der Universität Göttingen. Für FIM-/APT-Proben wurde das Material durch Funkenerosion in $0.3 \times 0.3 \times 20 \mathrm{~mm}^{3}$ große Blöcke geschnitten. Diese wurden dann in zwei Schritten mithilfe von Lösungen aus Perchlorsäure, Essigsäure und Ethylenglykolmonobutylether $\left(\mathrm{HClO}_{4}, \mathrm{C}_{2} \mathrm{H}_{4} \mathrm{O}_{2}\right.$ und $\left.\mathrm{C}_{6} \mathrm{H}_{14} \mathrm{O}_{2}\right)$ elektrochemisch gedünnt nach dem Verfahren von FROMMEYER [58]. Abschließend folgte eine Reinigung mit Methanol.

Für TEM-/ALCHEMI-Proben wurden aus dem Material mit dem Fokussierten Ionenstrahl (Focussed Ion Beam FIB) Lamellen von ca. $10 \mu \mathrm{m} \times 10 \mu \mathrm{m} \times 2 \mu \mathrm{m}$ mithilfe von Galliumionen herausgeschnitten, die dann mithilfe der Omniprobe herausgenommen wurden. Durch Platindeposition können diese an einen Halter angeklebt werden. Anschließend werden sie in mehreren Stufen gedünnt, bis der zentrale Bereich der Lamelle eine Dicke von unter 100nm aufweist. Der Galliumionenstrahl wird dabei mit immer geringeren Intensitäten und Beschleunigungsspannungen - zuletzt von 10kV - betrieben. Um zusätzlich das Material vor struktureller Veränderung zu schützen, ist eine Platinschutzschicht aufgebracht (siehe nebenstehend Abbildung 3.3).

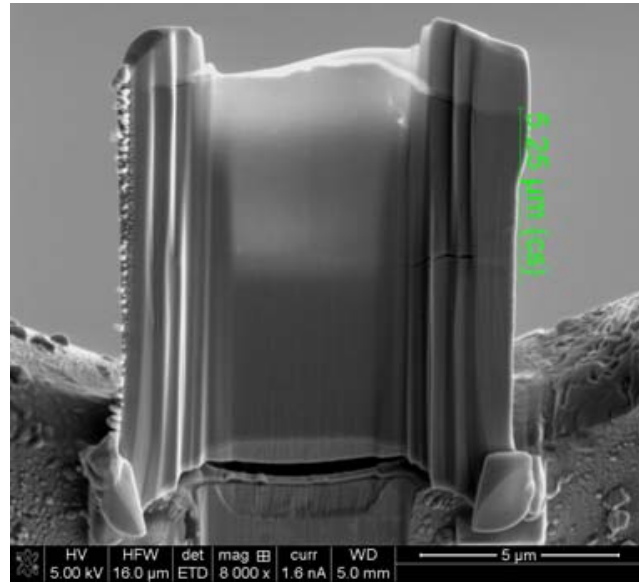

Abbildung 3.3: Fertig präparierte Lamelle für TEM-/ALCHEMI-Untersuchungen.

Ganz oben ist noch ein Teil der Platinschutzschicht zu erkennen.

ii Hinweis: Um die Lesbarkeit zu erhöhen, werden im Folgenden diese Kompositionen in der etwas ungenaueren Form „Fe ${ }_{3} \mathrm{Al}$-Zusatz" genannt. 


\subsubsection{Nanokristalline Materialien}

Die Messungen an nanokristallinem Fe ${ }_{3} \mathrm{Al}$ mit ternären Zusätzen wurden u. a. im Rahmen des DFGProjekts AL 592/3-1 durchgeführt. Dieses befasst sich im Wesentlichen mit der atomaren Gefügestruktur von $\mathrm{Fe}_{3} \mathrm{Al}-\mathrm{Cr}$ und $\mathrm{Fe}_{3} \mathrm{Al}-\mathrm{Ti}$, da diese Zusätze die stärksten Veränderungen in Duktilität und Stabilität hervorrufen (siehe 2.1). Daher wurde ein Schwerpunkt auf diese Konzentrationen gelegt.

Das nanokristalline Probenmaterial wurde ebenfalls am MPIE mithilfe von Kugelmühlen hergestellt. Dazu wurde das Ausgangsmaterial bereits pulverförmig mit den Stahlkugeln in den Attritor gegeben. Es wurden Probenchargen aus reinen Materialien, d.h. das Material wurde in der Kugelmühle mechanisch legiert, und aus bereits vorher legierten Proben hergestellt. Die Mahldauer wurde dabei empirisch angepasst und durch Röntgendiffraktometrie kontrolliert, entsprechende Messungen zeigt Abbildung 3.4. Bei den mechanisch legierten Proben wurde insbesondere auf die Homogenität geachtet, d.h. vorrangiges Ziel war dabei, solange zu mahlen, bis das XRD-Spektrum keine Peaks der reinen Eisenoder Aluminiumphase mehr aufweist. Dies war im Wesentlichen nach 30h Mahlen erreicht, für die hier verwendeten Proben wurden 50h gewählt, um eine einheitliche, ausreichende Mahldauer anzustreben. Die anderen Proben wurden so lange gemahlen, bis man anhand der XRD-Messungen durch Scherrer-Berechnungen [59] abschätzen konnte, dass eine annähernd minimale Korngröße erreicht wurde. Diese liegt demnach bei ca. $10 \mathrm{~nm}$ - 15nm, bei den mechanisch legierten Proben sind sie tendenziell geringfügig kleiner. So wurden diese Proben anfänglich $8 \mathrm{~h}$ gemahlen, es zeigte sich, dass $4 \mathrm{~h}$ ebenfalls ausreichend sind. Eine detailliertere Beschreibung der Präparation sowie umfangreichere Analysen aller Proben durch XRD-Messungen sind nachzulesen bei [60]. Alle Probenchargen werden im Folgenden noch einmal tabelliert:

\begin{tabular}{cccc}
\hline Probencharge & Komposition & Legierung & Mahldauer \\
\hline 1 & $69 \mathrm{at} \% \mathrm{Fe}, 26 \mathrm{at} \% \mathrm{Al}, 5 \mathrm{at} \% \mathrm{Cr}$ & mechanisch & $50 \mathrm{~h}$ \\
2 & $70 \mathrm{at} \% \mathrm{Fe}, 26 \mathrm{at} \% \mathrm{Al}, 4 \mathrm{at} \% \mathrm{Ti}$ & mechanisch & $50 \mathrm{~h}$ \\
3 & $70 \mathrm{at} \% \mathrm{Fe}, 26 \mathrm{at} \% \mathrm{Al}, 4 \mathrm{at} \% \mathrm{Cr}$ & thermisch & $4 \mathrm{~h}$ \\
4 & $69 \mathrm{at} \% \mathrm{Fe}, 26 \mathrm{at} \% \mathrm{Al}, 5 \mathrm{at} \% \mathrm{Mn}$ & thermisch & $4 \mathrm{~h}$ \\
5 & $70 \mathrm{at} \% \mathrm{Fe}, 26 \mathrm{at} \% \mathrm{Al}, 4 \mathrm{at} \% \mathrm{~V}$ & thermisch & $8 \mathrm{~h}$ \\
6 & $70 \mathrm{at} \% \mathrm{Fe}, 26 \mathrm{at} \% \mathrm{Al}, 4 \mathrm{at} \% \mathrm{Ti}$ & thermisch & $8 \mathrm{~h}$ \\
\hline
\end{tabular}

Tabelle 3.1: Übersicht der hergestellten nanokristallinen Proben

Die Pulver zeigen alle eine Partikelgröße von ca. $10 \mu \mathrm{m}$ - 50 $\mu \mathrm{m}$. Insbesondere ist zu beachten, dass anhand der XRD-Untersuchungen weder eine $\mathrm{B} 2$ - oder $\mathrm{DO}_{3}$-Ordnung, noch weitere Phasen ermittelt wurden (siehe Abbildung 3.4). 


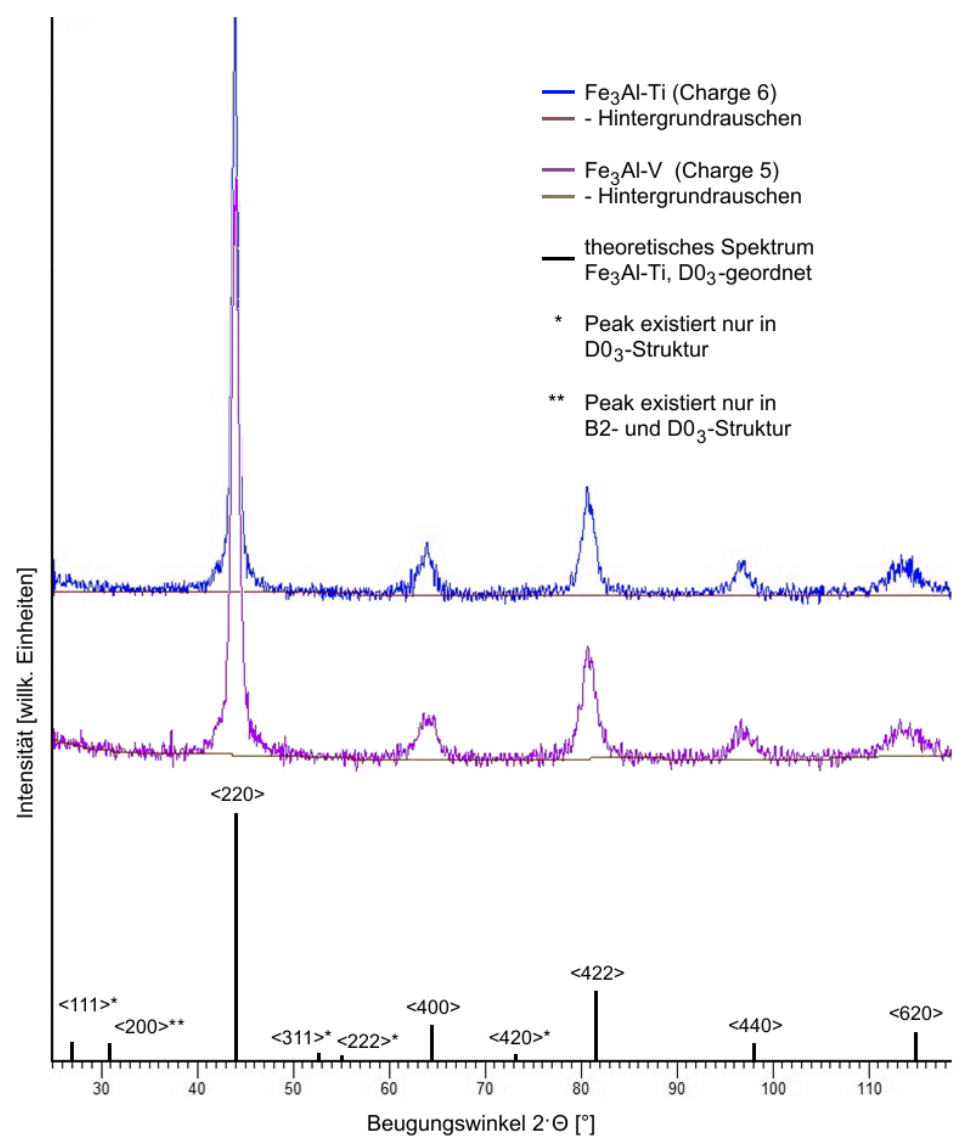

Abbildung 3.4: XRD-Spektren nanokristalliner Proben nach 8h Mahldauer (Messdaten von DEGES [60])

Die Präparation von nanokristallinem Pulver zu APT-Proben erfolgte wiederum am IMP Göttingen. Sie kann - ohne eine Konsolidierung des Materials - nur mit dem FIB geschehen. Die Vorgehensweise ist in Abbildung 3.5 veranschaulicht. Aus dem Pulver (a) wird eine Lamelle herausgeschnitten (b) und mithilfe der Omniprobe herausgenommen (c). Eine elektrochemisch vorpräparierte Wolframnadel (d) wird mit dem Galliumionenstrahl an der Spitze abgeflacht (e). Die Lamelle wird auf diese Nadel aufgebracht (f) und mit Platindeposition angeklebt. Der überstehende Teil der Lamelle wird abgeschnitten (g) und kann für weitere APT-Proben benutzt werden. Der aufgesetzte Block wird fest an der Nadel verankert. Dazu werden zunächst auf allen vier Seiten jeweils zwei Längsschnitte eingebracht (h), welche mit Platin aufgefüllt werden (i) und über die dann eine Platinschicht deponiert wird (j). Durch diese Prozedur wird die haftende Oberfläche zwischen Block und Wolfram im Vergleich zu einer Verbindung, die nur den Schritt (j) aufweist, erhöht. Außerdem werden auch größere Bereiche unterhalb des Blockes aufgefüllt, denn gerade das bei den Längsschnitten abgetragene Material redeponiert in Lücken zwischen Block und Spitze und füllt damit Bereiche, die aus geometrischen Gründen durch die Platindeposition allein nur schwer zu erreichen sind. Danach wird in mehreren Schritten im oberen Teil des Blockes seitlich Material abgetragen (k)-(m). Dabei werden die Galliumströme ständig reduziert. Ist der Block schmaler als ca. $0,3 \mu \mathrm{m}$, wird die Beschleunigungsspannung von $30 \mathrm{kV}$ auf $10 \mathrm{kV}$ verringert und weiterer Materialabtrag erfolgt bei $\pm 3^{\circ}$ verkippten Schnittkanten (n). Die letzten Schnitte erfolgen von 8 Seiten, durch die nicht zu vermeidende Streuung des Galliumstrahls entsteht dabei jedoch kein Achteck, sondern eine allseits abgerundete Spitze mit einem Krümmungsradius von deutlich weniger als $100 \mathrm{~nm}$ (o). 

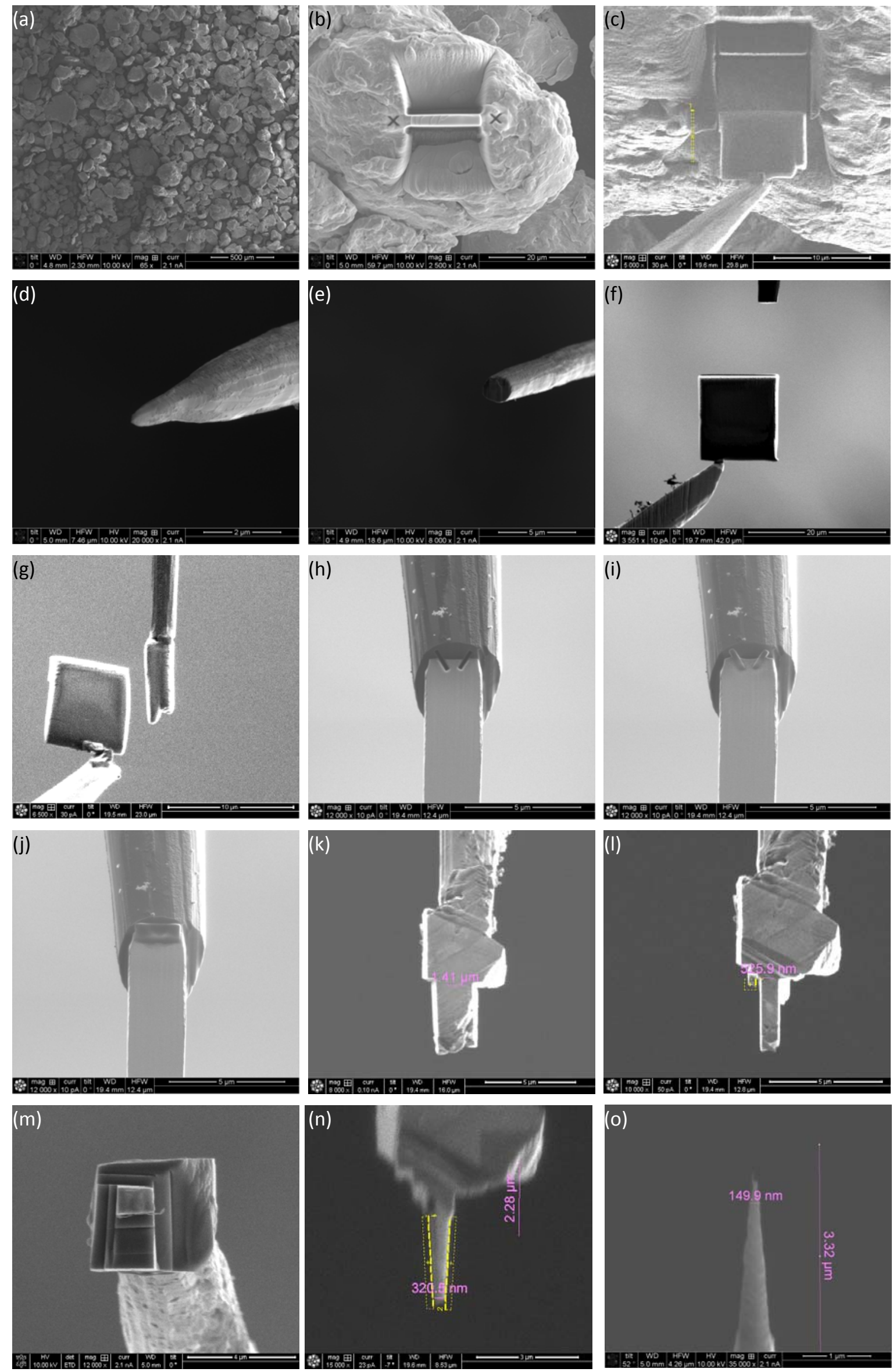

Abbildung 3.5: Präparation von APT-Proben mit dem FIB. Erläuterungen siehe Text. 


\subsection{Simulation von Atomsondenmessungen}

Bei der Rekonstruktion der Atompositionen aus den APT-Daten können Effekte auftreten, wodurch die Atompositionen verfälscht wiedergeben werden und dadurch eine direkte Aussage über die Platzbesetzung der Elemente behindert ist. Diese wurden in der Literatur schon häufig beschrieben [61, 62], für die vorliegende Arbeit erfolgen genauere Untersuchungen dazu in Kapitel 4.

Um diese Effekte zu verstehen und zu quantifizieren, wurde schon von BOLL und AL-KASSAB ein Programm entwickelt, das APT-Messungen an Titanbasislegierungen simuliert, wodurch Aussagen über die Platzbesetzung ternärer Elemente getroffen werden konnten [63]. Im Rahmen dieser Arbeit wurde ein Simulationsalgorithmus erarbeitet, der auf den Prinzipien der APT-Simulation von BOLL beruht. Neben der bestmöglichen Adaption an ternäre Eisenaluminide beinhaltet dieser Algorithmus eine individuellere Eingabe von Messparametern, sowie ein umfangreicheres Berechnungsmodell, insbesondere bei der Berechnung der Bindungsenergie.

\subsubsection{Allgemeines Arbeitsprinzip}

Das wesentliche Arbeitsprinzip besteht darin, dass für ein gegebenes Material, d.h. ein Volumen mit einer bestimmten Atomanordnung, das rekonstruierte Volumen einer APT-Messung, d.h. dessen Atomanordnung, simuliert wird.

Dazu muss als erstes die Probe erstellt werden, also Atompositionen und -massen einer virtuellen APTSpitze mit der benötigten Kristallstruktur und -orientierung bestimmt werden (ein sog. xyzmDatensatz). Diese Probe besteht aus einem geraden Kreiszylinder, der vorne abgerundet ist (mit dem Krümmungsradius $r_{k}$ ). Anschließend ist die Reihenfolge zu bestimmen, in der die Atome evaporieren, um daraus die Positionen im rekonstruierten APT-Volumen zu ermitteln. Die $x$ - und y-Komponente (also die lateralen Koordinaten) können unverändert bleiben, was einer Vergrößerung von 1 entspricht. Die z-Koordinate bestimmt man dann aus der Evaporationsreihenfolge der Atome, wie es in 3.1.1 beschrieben ist. Dabei wird ein konstanter Wert von $r_{k}$ angenommen, d.h. man simuliert also nach Formel 3.1 einen Messbereich konstanter Spannung $U$.

In jedem Simulationsschritt muss somit das Atom bestimmt werden, welches evaporiert. Dieses ist nach den Simulationsprinzip aus [63] dasjenige, welches sich aus energetischen Gründen am einfachsten ablösen lässt. Die Kohäsionsenergie $\mathrm{E}_{\mathrm{C}}$ ergibt sich dabei nach dem Müller-Schottky-Modell zur Feldevaporation allgemein aus mehreren Energiebeiträgen [48, 64]. Dafür gilt:

$$
\mathrm{E}_{\mathrm{C}}=\mathrm{E}_{\mathrm{B}}+\sum_{\mathrm{n}} \mathrm{I}_{\mathrm{n}}-\mathrm{n} \cdot \Phi+\mathrm{E}_{\mathrm{F}}+\mathrm{E}_{\mathrm{th}}
$$

Formel 3.8

Dabei ist $E_{B}$ die Bindungsenergie, $I_{n}$ die $n$-te lonisierungsenergie, $\Phi$ die Austrittsarbeit des Probenmaterials, $E_{F}$ der Energiebeitrag durch den Einfluss des elektrischen Feldes und $E_{t h}$ die thermische Energie.

Ist schließlich die Evaporationsreihenfolge der Atome bestimmt, kann das simulierte Volumen nach Formel 3.3 rekonstruiert werden. Abschließend werden die Atomkoordinaten noch mit einer Gaußfunktion verrauscht, um Messungenauigkeiten einzubeziehen. Die Stärke wird den Messdaten angepasst. Damit kann der simulierte xyzm-Datensatz ausgegeben werden und steht zur weiteren Analyse zur Verfügung (siehe auch [65]).

\subsubsection{Berechnung der Aktivierungsenergie $Q$}

Der genaue Effekt der Feldverdampfung ist ein komplexer Prozess, der bislang nur teilweise verstanden ist. Ein prinzipielles Modell geht auf MüLLER zurück [66]. Dabei wird die potentielle Energie eines Oberflächenatoms abhängig von seiner Position $\mathrm{x}$ senkrecht zur Oberfläche betrachtet. Für den Fall 
$\mathrm{F}=0$ entspricht diese dem Bindungspotential des Atoms. Im Fall eines anliegenden Feldes wird dieses durch ein Potential $V_{F}$ überlagert, welches aus einem Feldanteil und einem Bildladungsanteil besteht:

$$
V_{F}(x)=-n \cdot e \cdot F \cdot x+\frac{-n^{2} \cdot e^{2}}{16 \cdot \pi \cdot \varepsilon_{0} \cdot x}
$$

Zusätzlich erhöht sich die potentielle Energie um den Beitrag I, der zur lonisation nötig ist,

$$
I=\sum_{n} I_{n}-n \cdot \Phi
$$

Wie Abbildung 3.6 zeigt, ergibt sich durch dieses sog. Image-Hump-Modell ein Energieberg der Höhe $Q$ - der sog. Schottky-Hügel -, der zur Feldevaporation überwunden werden muss. Setzt man $Q$ gleich Null, so kann grundsätzlich die Verdampfungsfeldstärke F berechnet werden. Im Rahmen dieses Modells wird dieser Schottky-Hügel in einer gewissen Entfernung zur Oberfläche und zum Adatom angenommen. Dadurch kann der genaue Verlauf des Bindungspotentials vernachlässigt werden, es wird mit dem konstanten Wert von $E_{B}$ gerechnet. Effektiv bedeutet dies, dass zum Zeitpunkt des Ablösens $E_{B}$ schon komplett überwunden wurde. Vernachlässigt man thermische Energieeinflüsse, was aufgrund deren Werte gerechtfertigt ist, ist $\mathrm{Q}$ damit gleich $\mathrm{E}_{\mathrm{c}}$ aus Formel 3.8.

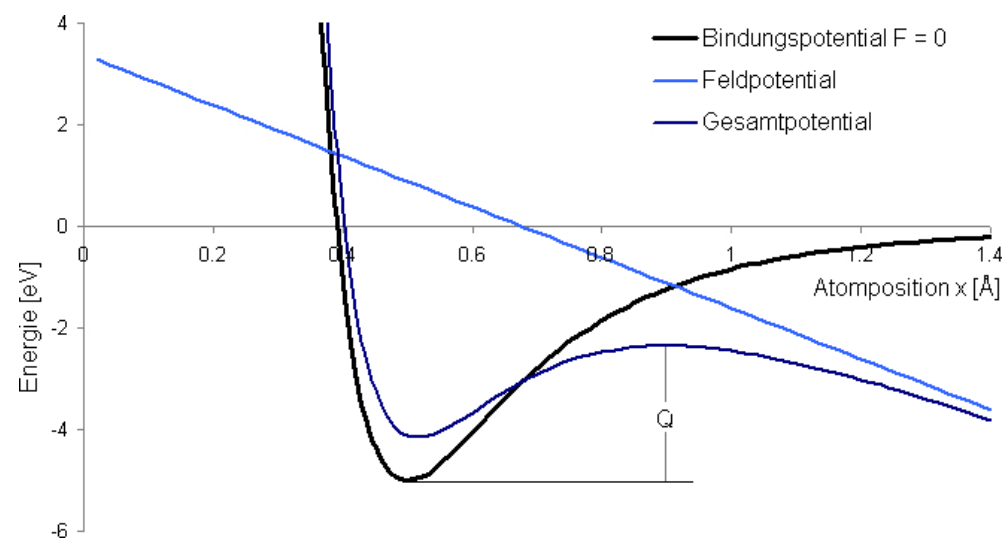

Abbildung 3.6: Schematische Potentialverläufe eines evaporierenden Atoms nach dem Image-Hump-Modell

Dieses Modell kann zwar die Feldverdampfungsfeldstärken einiger Metalle gut vorhersagen, ist allerdings teilweise unzureichend und wurde daher vielfach kritisiert [48,67]. Eine Erweiterung lieferten GOMER und SWANSON mit dem sog. Charge Exchange Modell [68]. Es verschiebt u.a. das Bildladungspotential um einen Wert $\mathrm{x}_{\mathrm{scr}}$ (screening distance - Abschirmungsabstand), um der nicht eindeutig zu benennenden Position der Oberfläche Rechnung zu tragen.

Demzufolge kommt es zu Potentialverläufen des lons, die den Potentialverlauf des neutralen Atoms an einer Stelle $x_{\text {int }}$ schneiden (siehe Abbildung 3.7), ab der die lonisierung des Atoms energetisch günstiger ist. Dadurch entsteht eine Aktivierungsenergie $Q$, die den Unterschied zwischen $V\left(x_{\text {int }}\right)$ und der neutralen Bindungsenergie darstellt.

Zur Berechnung von $Q$ und $x_{i n t}$ ist also die Kenntnis des genauen Potentialverlaufs von $E_{B}(x)$ erforderlich. Dieser kann zwar durch das EAM-Konzept (siehe 3.3.3) modelliert werden, genaue, quantitative Aussagen sind aber trotzdem diffizil und schon allein wegen der Nichtkenntnis von $\mathrm{x}_{\text {scr }}$ auszuschließen.

Stattdessen soll ein empirischer Ansatz gewählt werden, der das Berechnungsmodell von BOLL und AL-KASSAB [63] nur geringfügig verändert: $Q$ entspricht der Bindungsenergie $E_{B}$, vermindert um eine Energie $E_{F}$, die durch das Feld verursacht wird. Zusätzlich müssen noch die lonisationsenergie und die Austrittsarbeit aufsummiert werden. An dieser Stelle ist aber zu beachten, dass I nicht komplett addiert werden darf. Abbildung 3.7 zeigt dazu die Potentialverläufe eines Adatoms für zwei verschiedene Ioni- 


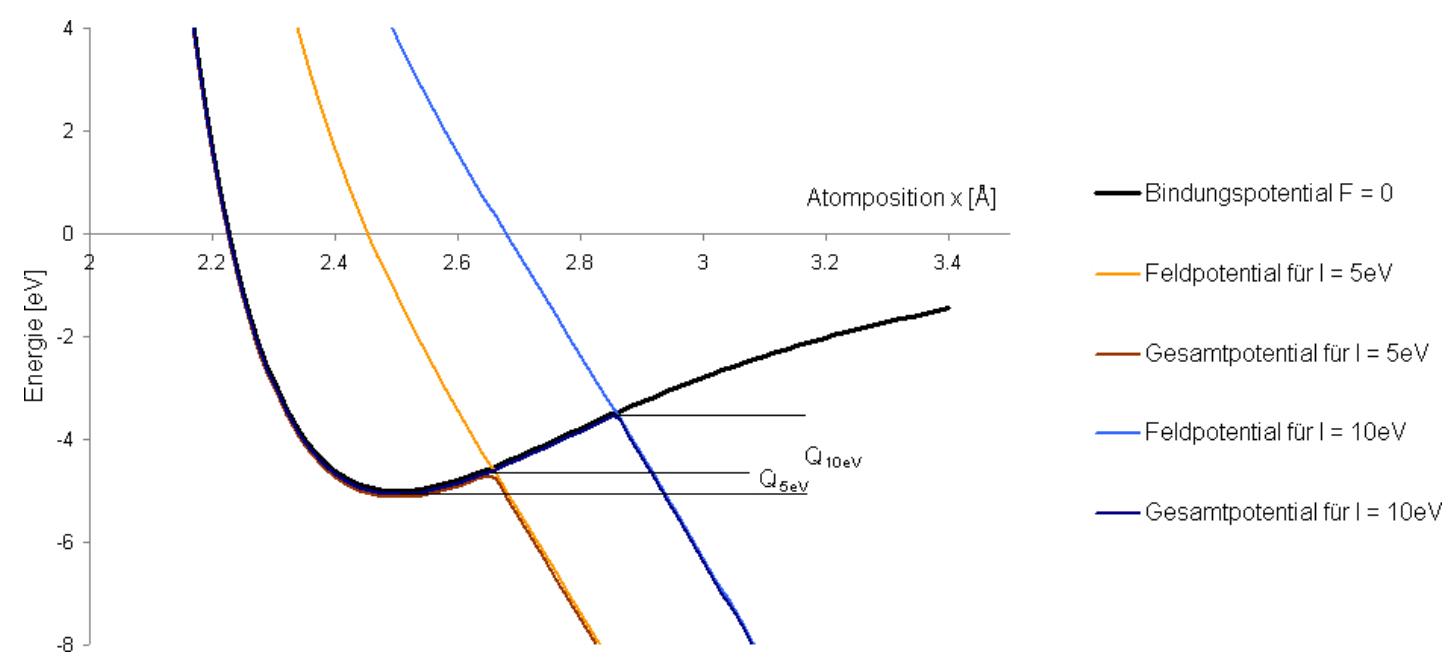

Abbildung 3.7: Potentialverläufe eines evaporierenden Atoms nach dem Charge-Exchange-Modell

sierungsenergien: $5 \mathrm{eV}$ und $10 \mathrm{eV}$ unter den (realistischen) Bedingungen $E_{B}=5 e V, F=30 V / \AA ̊ A, ~ n=1$. Es ist augenscheinlich zu erkennen, dass die Differenz von $Q_{5 e v}$ und $Q_{10 e v}$ etwa $1 \mathrm{eV}$ beträgt, obwohl die Differenz der lonisierungsenergien bei $5 \mathrm{eV}$ liegt. Die genaue Erhöhung $\Delta Q$ durch die Berücksichtigung von I ist also stark abhängig vom Potentialverlauf $E_{B}$ und analytisch für viele komplexe Potentialverläufe nicht zu berechnen. Allerdings kann sie für einen gewissen Wertebereich als linear angenommen wer den, so dass $\Delta \mathrm{Q}=\mathrm{K} \cdot \mathrm{I}$ gilt, mit dem empirisch zu ermittelnden Koeffizienten $\mathrm{K}$.

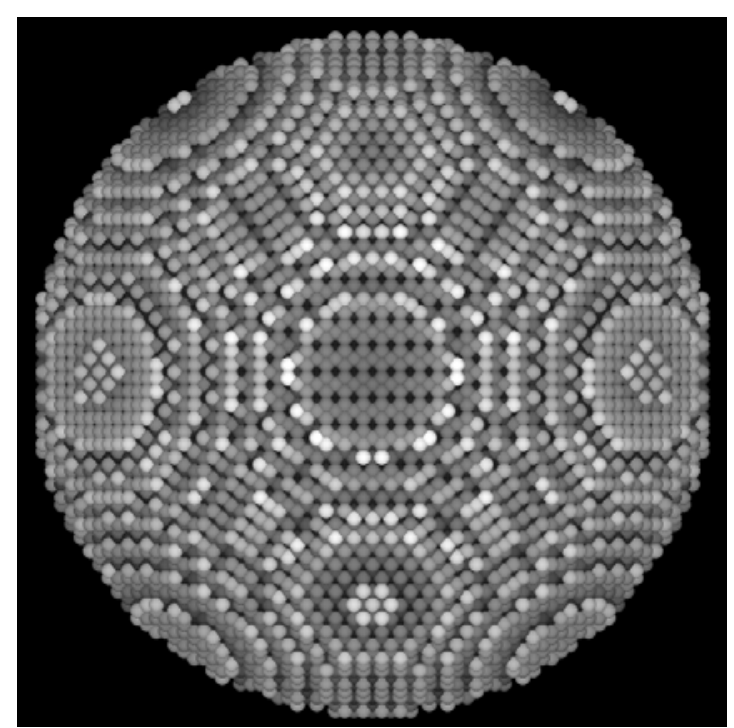

Abbildung 3.8: Aufsicht auf simulierte Probenspitze: Energetischer Einfluss des Feldes. Hell = starke Absenkung von $Q$
Die Bestimmung von $\mathrm{E}_{\mathrm{F}}$ wäre prinzipiell über die Feldstärke $\mathrm{F}$ möglich. Diese Vorgehensweise hat aber zwei Nachteile: $F$ im Raum über der Spitze müsste z.B. über eine Finite-Elemente-Methode bestimmt werden, die den Rechenaufwand stark ansteigen lässt. Außerdem kann - nach obiger Beschreibung - der quantitative Zusammenhang zwischen $F$ und $E_{F}$ nicht so genau bestimmt werden, dass sich dieser Mehraufwand lohnt.

Stattdessen wird ein einfacherer, empirischer Ansatz gewählt: $E_{F}$ ist (absolut) am höchsten an geometrischen Kanten und Eckpositionen von evaporierenden Kristallebenen, versteckte Atome erfahren einen geringeren Einfluss. Folglich ist $\mathrm{E}_{\mathrm{F}}$ eine Funktion des Abstandes $\mathrm{x}$ zur globalen (durchschnittlichen) Probenoberfläche, die in dieser Arbeit beschrieben wird durch:

$$
E_{F}=D \cdot x^{2}
$$

Formel 3.11

Dabei ist $D$ ein empirisch zu bestimmender Koeffizient. Der Einfluss von $E_{F}$ ist schematisch in Abbildung 3.8 visualisiert. Die Abbildung erinnert an ein FIM-Bild. Das bedeutet, der energetische Einfluss nach Formel 3.11 ist dort am stärksten, wo auch in einem FIM-Bild am meisten Bildgasionisation auftritt und damit das Feld am höchsten ist. Da Q durch das Feld nur abgesenkt werden kann, müsste Formel 3.11 noch um eine additive Konstante ergänzt werden, so dass (für realistische $x$-Werte) $E_{F}$ stets kleiner Null ist. Da während der Simulation beim Vergleich der Energien aller Atome diese Konstante keine Auswirkungen hat, kann sie an dieser Stelle weggelassen werden. 
Insgesamt ergibt sich damit die Formel

$$
\mathrm{Q}=\mathrm{E}_{\mathrm{B}}+\mathrm{K} \cdot\left(\sum_{\mathrm{n}} \mathrm{I}_{\mathrm{n}}-\mathrm{n} \cdot \Phi\right)+\mathrm{D} \cdot \mathrm{x}^{2}+\mathrm{E}_{\mathrm{th}}
$$

Die thermische Energie $E_{t h}$ ist zwei Größenordnungen kleiner als die anderen Energiebeiträge und könnte daher prinzipiell vernachlässigt werden. Für die Simulation ist sie dennoch aus praktischen Gründen brauchbar, da ihr zufälliger Wert ein gewisses zufälliges Verhalten in den Evaporationsablauf ein bringt.

Zu erwähnen ist, dass das oben beschriebene Charge-Exchange-Modell zwar allgemeiner als das ImageHump-Modell ist, dennoch können auch hier noch Energiebeiträge ergänzt werden. So erwähnten GOMER und SWANSON [68] Einflüsse der Polarisierbarkeit und des Dipolmomentes des Adatoms, sowie veränderte Energielevel der übertragenen Elektronen. Allerdings sind viele dieser Effekte quantitativ schwer zu erfassen. Weil sie im Allgemeinen deutlich kleiner sind als die anderen Energiebeiträge, werden sie daher hier - genauso wie in vielen anderen Berechnungen - vernachlässigt.

Anzumerken ist auch, dass durch obige Formel und Simulation keine Aussagen über den eigentlichen Evaporationsprozess getroffen werden können. Beispielsweise hat FORBES [67] angemerkt, dass die Vorstellung des plötzlichen Ladungsüberganges unrealistisch sei und stattdessen ein Fließen der Ladung den Vorgang besser umschreibe und fügte einen Vorfaktor - vergleichbar mit $\mathrm{K}$ aus Formel 3.12 ein. Er prägte daher den Begriff charge drain und es gibt Abschätzungen über energetische Einflüsse daraus [69]. Die vorliegende Formel 3.12 schließt solche Einflüsse dabei nicht aus. Durch die Benutzung zweier empirisch zu ermittelnder Konstanten ( $D$ und K), können fehlerhafte Betrachtungen der energetischen Verhältnisse durch ein vereinfachtes Bild der Feldevaporation (teilweise) kompensiert werden. Gleichzeitig erlaubt die Anzahl von nur zwei Konstanten eine gangbare Eichung dieser durch den Vergleich von Simulationen und Messungen. Weitergehende Betrachtungen und Diskussionen dazu sind in 8.2 zu finden.

\subsubsection{Berechnung der Bindungsenergie mithilfe der Embedded Atom Method}

Ein wesentliches Merkmal des in dieser Arbeit verwendeten Simulationsalgorithmus ist die detaillierte Berechnung der Bindungsenergie. In bisherigen APT-Simulationen [63] wurde diese nur durch feste Bindungsbeiträge zwischen benachbarten Kristallatomen berechnet. Eine solche Methode, oder auch die Berechnung nur durch Paarpotentiale, hat den Nachteil, Bindungsverhältnisse im Allgemeinen nur unzureichend zu beschreiben. So kann dieser Ansatz beispielsweise den Unterschied zwischen der Sublimations- und der Leerstellenenergie nicht erklären und auch Bindungsverhältnisse an Grenz- und Oberflächen zeigen ungenügende Genauigkeit.

Daher wurde für diese Arbeit $\mathrm{E}_{\mathrm{B}}$ mithilfe der Embedded Atom Method (EAM) berechnet. Die Bindungsenergie ist dabei nach FINNIS und SINCLAIR [70] aus einer Summe von Paarpotentialen und einem Term, der dem kollektiven Einfluss der Umgebungsatome Rechnung trägt, zu bilden. Letzterer wird über den Elektronendichteeinfluss benachbarter Atome bestimmt und setzt dabei die Prinzipien der quantenmechanischen Dichtefunktionaltheorie im Rahmen eines empirischen Modells ein. Für ein Atom i gilt:

$$
E_{B, i}=\sum_{j} \frac{1}{2} \cdot V\left(r_{i, j}\right)+F\left(\rho_{i}\right)
$$

$\mathrm{V}\left(\mathrm{r}_{\mathrm{i}, \mathrm{j}}\right)$ ist dabei das Paarpotential zwischen den Atomen $\mathrm{i}$ und $\mathrm{j}$ mit dem Abstand $\mathrm{r}_{\mathrm{i}, \mathrm{j}}, \rho_{\mathrm{i}}$ ist die Elektronendichte an der Position i und $F(\rho)$ die Einbettungsfunktion. Nach $[71,72]$ kann $\rho_{\mathrm{i}}$ als Summe aus den Beiträgen der Umgebungsatome gebildet werden: 


$$
\rho_{\mathrm{i}}=\sum_{\mathrm{j}} \rho_{0, \mathrm{j}} \cdot\left(\mathrm{d}_{\mathrm{NN}, \mathrm{j}} / \mathrm{r}_{\mathrm{i}, \mathrm{j}}\right)^{6}
$$

Dabei ist $d_{N N, j}$ der Abstand des nächsten Nachbarn (NN) der puren Phase des Elementes $j$ und $\rho_{0}$ ein elementspezifischer Skalierungsfaktor. Dieser ist abhängig von der Leerstellenbildungsenergie $E_{L S}$, der Sublimationsenergie $\mathrm{E}_{\text {sub }}$ und dem Atomvolumen $\Omega$, wiederum jeweils des reinen Elements [72]:

$$
\rho_{0, j}=\left(E_{\text {sub }}-E_{L S} / \Omega\right)^{3 / 5}
$$

Formel 3.15

Im Rahmen dieser Arbeit wurde für die Einbettungsfunktion $\mathrm{F}(\rho)$ die Wurzelfunktion verwendet nach der Beschreibung von [70]. Der Koeffizient dieser Funktion ist dadurch determiniert, dass die halbe Gesamtbindungsenergie eines Atoms nach Formel 3.13 seiner Sublimationsenergie entsprechen muss.

Anzumerken ist, dass mit der Berechnung von $E_{B}$ nach Formel 3.13 der Algorithmus deutlich komplizierter ist, als wenn nur Paarpotentiale benutzt werden. Zum einen, da mit dem zweiten Term aus Formel 3.13 das Potential zwischen zwei Atomen nicht mehr symmetrisch ist, zum anderen, da durch die Wurzelfunktion die Abhängigkeit von den NN nicht mehr linear ist. Praktisch bedeutet dies, dass beim Evaporieren eines Atoms die Energie $E_{B}$ der verbliebenen Nachbaratome nicht um einen bestimmten Wert gesenkt werden kann, sondern deren Bindungsenergie vollkommen neu berechnet werden muss.

Die EAM ist also ein komplexes Modell zur Berechnung der Bindungsenergie. Dabei werden allerdings nur bekannte bzw. makroskopisch relativ leicht zu ermittelnde Konstanten benutzt (wie die Leerstellenbildungsenergie $E_{L S}$, die Sublimationsenergie $E_{s u b}$, das Atomvolumen $\Omega$ und der NN-Abstand $d_{N N}$ ). Diese verwendeten Daten sind in Tabelle B.1 im Anhang aufgelistet. Einzig das Paarpotential muss bestimmt werden, dies geschieht im Allgemeinen z.B. durch Vergleich und Anpassen an Gitter- und elastische Konstanten. Es gibt in der Literatur etliche solcher Arbeiten, wodurch eine Vielzahl an Paarpotentialen zur Verfügung steht. Teilweise sind jedoch gerade intermetallische Paarpotentiale nicht vorhanden oder diese sind in verschiedenen Arbeiten stark unterschiedlich.

In dieser Arbeit wurden die Paarpotentiale durch das Lennart-Jones-Potential beschrieben:

$$
V(r)=\varepsilon\left\{\left(\frac{r_{0}}{r}\right)^{12}-2 \cdot\left(\frac{r_{0}}{r}\right)^{6}\right\}
$$

Dessen Minimum liegt bei $\left(r_{0},-\varepsilon\right)$. Des Weiteren wurden für die vorliegenden Berechnungen $V(r)$ und $\rho(r)$ bis zu den dritten NN miteinberechnet $\left(r_{i, j}<4,1 \AA ̊\right)$, auch dies ist eine Erweiterung des Simulationsalgorithmus von [63].

\subsubsection{Weitere Neuerungen des Simulationsprogramms}

\section{Berücksichtigung der Projektion}

Das simulierte Volumen hat eine Vergrößerung von 1, weshalb die $\mathrm{x}$ - und $\mathrm{y}$-Koordinaten der Atompositionen bislang unverändert blieben. Allerdings wird dabei der Projektionseffekt vernachlässigt.

In der Rekonstruktion von APT-Messungen (3.1.1) wird das Detektorsignal auf die Zwischenebene projiziert. Eine weitere Korrektur zur Beachtung der gekrümmten Oberfläche wird vernachlässigt, da sie kleiner als die Messgenauigkeit wäre. Gleiches gilt für den entsprechenden zusätzlichen Einfluss auf die z-Komponente.

In der Simulation wurde nun auch diese „Vernachlässigung" berücksichtigt, um das gleiche Berechnungsprinzip wie in den Messrekonstruktionen zu verwenden. Damit werden Simulationen und Mes- 
sungen noch wirklichkeitsnäher miteinander verglichen. Dass dies sinnvoll ist, zeigt eine Abschätzung ${ }^{\text {iii. }}$ Zwar liegen die maximalen Einflüsse mit $\Delta x=\Delta y \approx 2,8 \AA$ bzw. $\Delta z \approx 1,2 \AA$ wie bereits erwähnt im Bereich der Messgenauigkeit, sie können sich aber innerhalb der AtomVicinity-Profile aufgrund deren höchster Signifikanz durchaus bemerkbar machen (siehe auch 8.2.2).

\section{Individuelle Eingabe von $\mathbf{r}_{\mathbf{k}}$}

Eine weitere Verbesserung hin zu einer genaueren Simulation ist die separate Angabe des Krümmungsradius $r_{k}$. In den APT-Simulationen von BOLL [63] entspricht dieser automatisch dem Schaftradius $r_{s}$, so dass der vordere Teil des Volumens eine Halbkugel darstellt. Mit einem unabhängigem $r_{k}$ kann ein realistischeres Probenvolumen entworfen werden. Denn aus Formel 3.2 und Formel 3.3 folgt:

$$
\frac{r_{s}}{r_{k}}=\frac{r_{\text {det }} \cdot \eta}{L} \quad \text { mit } \quad r_{s}=\sqrt{x^{2}+y^{2}} \quad \text { bzw. } \quad r_{\text {det }}=\sqrt{x_{\text {det }}^{2}+y_{\text {det }}^{2}}
$$

Formel 3.17

Das heißt also, dass die Radien $r_{s}$ und $r_{k}$ in einem festen Verhältnis zueinander stehen, welches - nach den Prinzipien aus 3.1.1 - nur von den Geometrien des Atomsondenaufbaus abhängt.

Dieser Aspekt ist durchaus wichtig, da $r_{k}$ nach Formel 3.3 und Abbildung 3.2 einen direkten Einfluss auf die z-Komponente hat. Entsprechende Auswirkungen werden in 4.6 untersucht.

Abbildung 3.9: Schematische Darstellung der Form der virtuellen APT-Spitze mit dem Schaftradius $r_{s}$ und dem vorderen Krümmungsradius $r_{k}$

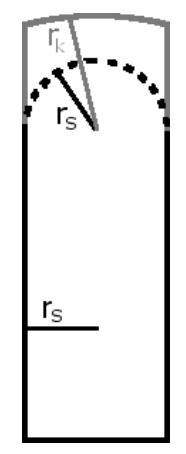

\subsection{Das ALCHEMI-Verfahren}

Diese Untersuchungsmethode bietet die Möglichkeit, mithilfe eines Transmissionselektronenmikroskops Atompositionen in einer Kristallstruktur zu bestimmen. Bei dem von SPENCE und TAFT $\varnothing$ vorgestellten ALCHEMI-Verfahren (Atom Location by Channeling Enhanced Microanalysis - Atomlokalisierung durch Kanalisierungsunterstützte Mikroanalyse) wird das Channeling von Elektronen entlang der Kristallebenen ausgenutzt, um eine unterschiedlich starke Röntgenemission verschiedener Kristallpositionen hervorzurufen $[73,74]$.

Man orientiert dabei die Probe nahe der Braggbedingung, die Elektronen, die sich durch den Kristall bewegen, können im Rahmen der dynamischen Theorie durch Blochwellen beschrieben werden. Wird der Einstrahlwinkel davon ausgehend verkleinert (Ablenkungsparameter $s<0$ ), bewegen sie sich zunehmend entlang der Atomlagen des Kristalls; wird er vergrößert ( $s>0)$, bewegen sie sich dazwischen (siehe Abbildung 3.10). Deswegen entstehen in unterschiedlichen Atomlagen unterschiedlich starke Elektronendichten. Da durch inelastische Streuung mit den Atomen diese angeregt werden und Röntgenquanten emittieren, entsteht ein charakteristisches EDX-Spektrum (energy dispersive X-ray spectroscopy - Energiedispersive Röntgenspektroskopie).

\footnotetext{
iii Diese Abschätzung befindet sich im Anhang (C.1).
} 

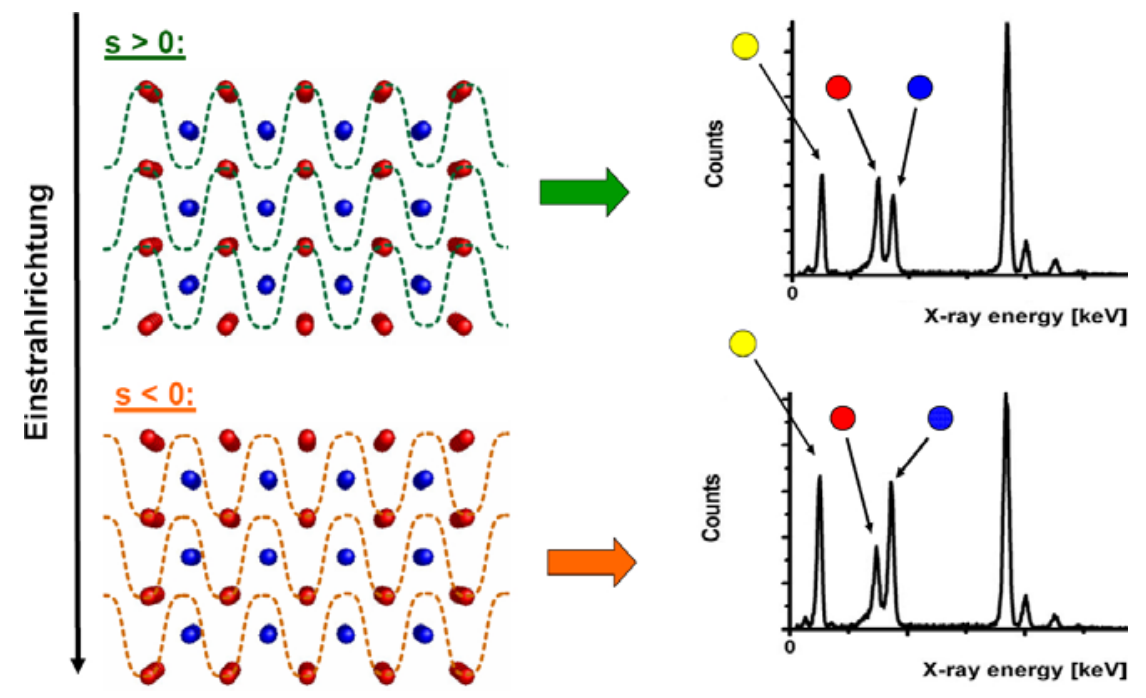

Abbildung 3.10: Prinzip der ALCHEMI-Methode.

Unterschiedliche Elektronendichten führen zu unterschiedlichen EXD-Spektren (nach LIEBSCHER [75]).

Aufgrund der unterschiedlich starken Anregung bestimmter Atompositionen in beiden Fällen ( $s>0$ und $s<0$ ) kann das Verhalten von ternären Elementen Aufschluss über die Platzbesetzung geben: In dem Beispiel aus Abbildung 3.10 verhalten sich die EDX-Intensitäten der gelben Atome wie die der blauen Atome und gegensätzlich zur denen der roten Atome. Daher kann eine Platzbesetzung von blau durch gelb geschlussfolgert werden. Für quantitative Analysen aus den jeweiligen Intensitäten I und I' beider Messungen muss deren Abhängigkeit von den Elektronenstromdichten $\mathrm{j}$ betrachtet werden. Für die Atomsorten $A$ und $B$ sowie dem ternären Zusatz $X$ gilt:

$$
\begin{array}{lll}
I_{A}=k \cdot C_{A} \cdot n_{A} \cdot j_{A} & I_{B}=k \cdot C_{B} \cdot n_{B} \cdot j_{B} & I_{X}=k \cdot C_{X} \cdot n_{X} \cdot\left(\xi_{X, A} \cdot j_{A}+\left(1-\xi_{X, A}\right) \cdot j_{B}\right) \\
I_{A}^{\prime}=k^{\prime} \cdot C_{A} \cdot n_{A} \cdot j_{A}^{\prime} & I_{B}^{\prime}=k^{\prime} \cdot C_{B} \cdot n_{B} \cdot j_{B}^{\prime} & I_{X}^{\prime}=k^{\prime} \cdot C_{X} \cdot n_{X} \cdot\left(\xi_{X, A} \cdot j_{A}^{\prime}+\left(1-\xi_{X, A}\right) \cdot j_{B}^{\prime}\right)
\end{array}
$$

Formel 3.18

Dabei sind k, k' Intensitätsfaktoren der Messung, $n$ die jeweiligen Anteile der Atomsorten auf den Ebenen und $\mathrm{C}$ die jeweiligen Faktoren, die die unterschiedlichen lonisationswahrscheinlichkeiten, Fluoreszenzausbeuten und andere Skalierungsfaktoren beinhalten. Mithilfe der sog. Doppelverhältnisbildung kann der Grad der Platzbesetzung $\xi_{X, A}$ von X-Atomen auf A-Plätzen derart berechnet werden, dass sich diese Koeffizienten aufheben:

$$
\frac{I_{X}}{I_{X}^{\prime}}=\xi_{x, A} \cdot \frac{I_{A}}{I_{A}^{\prime}}+\left(1-\xi_{x, A}\right) \cdot \frac{I_{B}}{I_{B}^{\prime}} \quad \Rightarrow \quad \xi_{X, A}=\frac{\frac{I_{X}}{I_{X}^{\prime}}-\frac{I_{B}}{I_{B}^{\prime}}}{\frac{I_{A}}{I_{A}^{\prime}}-\frac{I_{B}}{I_{B}^{\prime}}}
$$

In dieser Formel wird von einer Channeling- und einer Randomrichtung ausgegangen, so dass für die Elektronenstromdichten $\vec{j}_{A}^{\prime}=\vec{j}_{B}^{\prime}$ gilt [76]. Diese Analysemethode ist insbesondere für geringe Konzentrationen der ternären Zusätze sinnvoll und durch die Auswertung, die auch ohne Kenntnis der Strukturfaktoren möglich ist, vergleichsweise einfach. 


\section{Kapitel 4: Allgemeine Analyse von FIM- und APT-Messungen}

In diesem Kapitel werden FIM- und APT-Messungen auf allgemeine Eigenschaften untersucht und generelle Analysen durchgeführt. Ein Schwerpunkt liegt dabei auf der Charakterisierung der Messeffekte und -eigenheiten der APT-Daten. Daran angelehnt, sollen APT-Simulationen der binären Legierung durchgeführt und kalibriert werden.

\subsection{FIM-Resultate}

Abbildung 4.1 zeigt eine FIM-Aufnahme der binären Legierung. Dieses Bild wurde nach einer APTMessung bei $15 \mathrm{kV}$ aufgenommen. Durch die Messung ist eine stetige Feldverdampfung erfolgt, wodurch die Probe gleichmäßig abgerundet ist und man daher ein gleichmäßig entwickeltes und unverzerrtes FIM-Bild erhält.

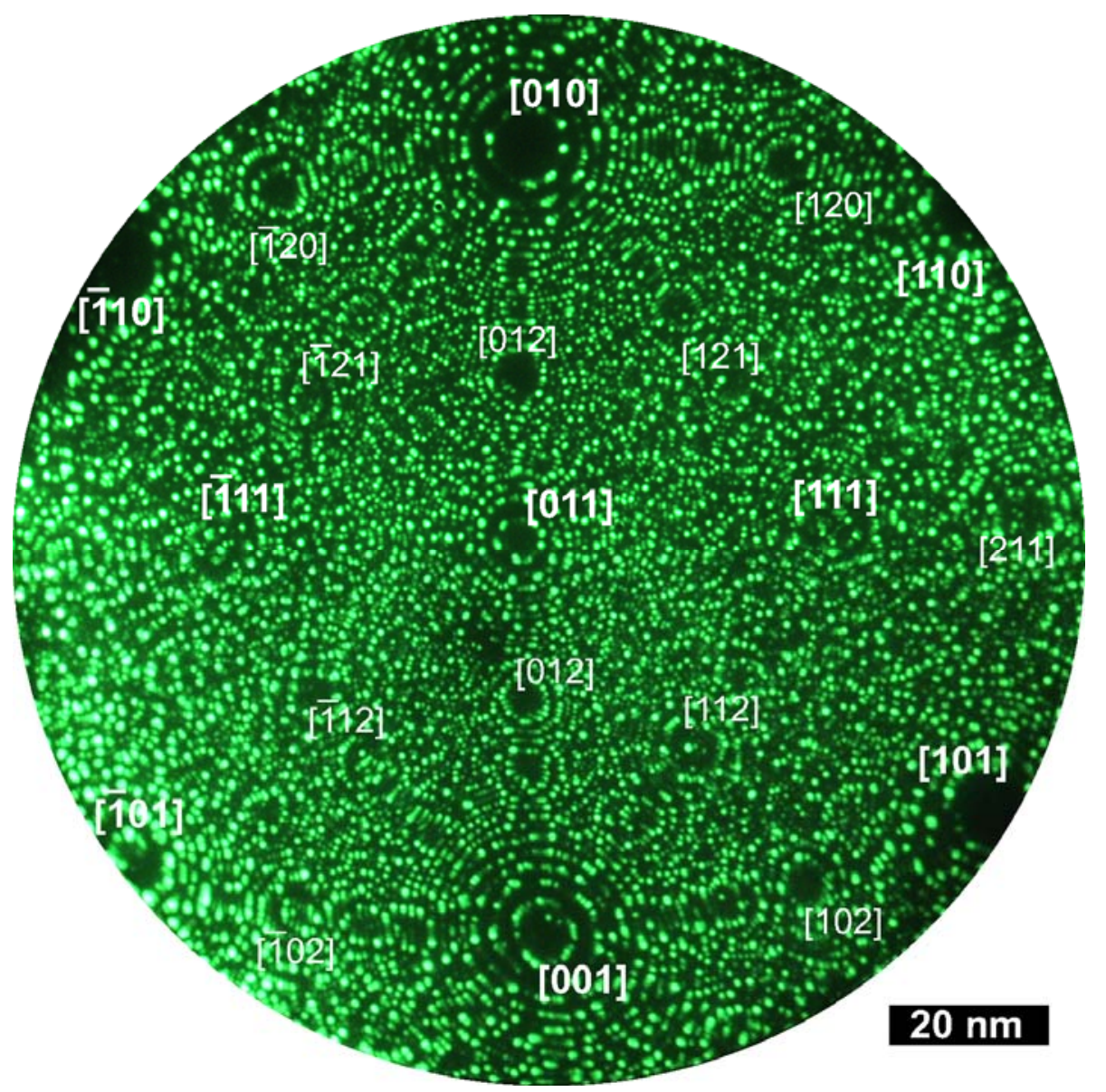

Abbildung 4.1: FIM-Bild von $\mathrm{Fe}_{3} \mathrm{Al}$ bei $20 \mathrm{~K}$ und $15 \mathrm{kV}$.

Pole sind indiziert, Hauptrichtungen hervorgehoben.

Es können klar Pole niedriginduzierter Richtungen ausgemacht werden. Am deutlichsten sind die $<100>$-Pole zu erkennen, ebenfalls treten die Pole der <110>-Richtungen vernehmlich hervor. Dies ist damit zu erklären, dass diese Richtungen die größten Ebenenabstände aufweisen. Dadurch bilden sich bei abgerundeten Proben an diesen Stellen die Stufen der Kristallstruktur am deutlichsten heraus. Die 
Pole der <111>-Orientierung sind daher auch nicht so klar zu erkennen, in Abbildung 4.1 sind sie kaum auszumachen, etwas deutlicher sind sie beispielsweise in Abbildung 4.2 zu erkennen.

Das bedeutet, dass die Orientierung der Proben dadurch eindeutig bestimmt und dass eine APTMessung an dieser Probe damit klar einer definierten Richtung zugeordnet werden kann.

Ebenfalls ist eine Überprüfung, ob Gitterdefekte an der untersuchten Stelle auftreten, möglich. Von ca. 40 Proben, an denen FIM- und/oder APT-Messungen vorgenommen wurden, konnte nur in einem Fall eine Korngrenze ausgemacht werden. Damit kann bestätigt werden, dass die intensive Auslagerung zur Optimierung der Homogenität der Proben (siehe 3.2.1) die erwünschten Effekte erzielt haben.

In FIM-Bildern können unterschiedliche Elemente nur aufgrund ihres Kontrastes unterschieden werden. In $\mathrm{Fe}_{3} \mathrm{Al}$ ist das erkennbare Element im Wesentlichen Aluminium. Das kann durch ein Verfahren basierend auf geometrischen Überlegungen nach WENG überprüft werden [77]. Dazu werden für mindestens zwei Hauptrichtungen die in FIM-Bildern erkennbaren (relativen) Ebenenabstände ermittelt. Nun ist zu überlegen, welche Abstände jeweils zu erkennen sein müssten, wenn nur Aluminium, nur Eisen oder beides die identifizierbaren Atome in FIM-Bildern darstellen. Durch einen Vergleich dieser Ebenenabstände, kann das abbildende Element bestimmt werden. Die entsprechenden Daten sind in Tabelle 4.1 aufgelistet, inklusive der Messung an Abbildung 4.1, dessen Ebenenabstände in Einheiten des Krümmungsradius angegeben sind. Offensichtlich zeigen diese Daten, dass Aluminium als das abbildende Element in $\mathrm{Fe}_{3} \mathrm{Al}$ konstatiert werden kann, womit die Ergebnisse von WENG bestätigt werden (siehe auch [58]).

\begin{tabular}{cccc}
\hline & Verhältnis des Ebenenabstandes $\mathbf{d}_{<110\rangle} / \mathbf{d}_{<100\rangle}$ & \\
& theoretisch & & gemessen \\
\hline wenn nur & wenn nur & wenn Eisen und & \\
Aluminium abbildet & Eisen abbildet & Aluminium abbilden & \\
\cline { 1 - 3 } $2,04 \AA / 2,89 \AA$ & $2,04 \AA / 1,445 \AA$ & $2,04 \AA / 1,445 \AA$ & $0,00320 r_{k} / 0,00428 r_{k}$ \\
$\sim 0,706$ & $\sim 1,412$ & $\sim 1,412$ & $\sim 0,748$ \\
\hline
\end{tabular}

Tabelle 4.1: Ebenenabstände in FIM-Aufnahmen

Das bedeutet, dass die Feldionisation des Bildgases also an Aluminiumatomen stattfindet, dort ist die Feldstärke folglich am höchsten. Demzufolge hat in $\mathrm{Fe}_{3} \mathrm{Al}$ das Aluminium also die höhere Feldverdampfungsfeldstärke. Das ist zunächst überraschend, da in den jeweils reinen Phasen die Evaporationsfeldstärke von Aluminium deutlich kleiner ist als diejenige des Eisens [48]. Man kann also daraus schließen, dass sich in $\mathrm{Fe}_{3} \mathrm{Al}$ die Bindungsverhältnisse ändern und damit das Aluminium die höchste Feldverdampfungsfeldstärke aufweist.

Prinzipiell können FIM-Aufnahmen auch dazu dienen, die Ordnung/Platzbesetzung von Zusätzen zu untersuchen, sofern diese Elemente durch ihren Kontrast ausgemacht werden können. In Abbildung 4.2 ist dazu als Beispiel ein FIM-Bild einer $\mathrm{Fe}_{3} \mathrm{Al}$-V-Legierung dargestellt. Betrachtet man die Ringe um die $<100>$-Pole, können einzelne Punkte (Atome) mit auffälligem Helligkeitswert identifiziert werden. Beim Vergleich mit <100>-Polen eines FIM-Bildes der binären Legierung (Abbildung 4.1) ist aber festzustellen, dass solche Helligkeitsunterschiede dort auch zu erkennen sind. Auf ternäre Zusätze sind diese also nicht zurückzuführen, es ist anzunehmen, dass fehlpositionierte Atome oder die lokale Probengeometrie die Ursache dafür sind. Auch an <111>-Polen sind entsprechende Erkenntnisse kaum zu gewinnen. Zwar sind in dieser Richtung reine Aluminiumebenen vorhanden (vergl. Abbildung 4.5), allerdings sind hier die einzelnen Ebenen nicht besonders gut zu erkennen. Eine (verminderte) Bildgasioni- 
sation an Eisenatomen kann hier aufgrund des geringen Ebenenabstandes nicht ausgeschlossen werden. Dadurch ist es nicht möglich, eine ausgeprägte Ringstruktur zu erkennen, wie die FIM-Bilder zeigen; stattdessen sinkt der Kontrast. Damit werden ein eindeutiges Erkennen von ternären Atomen und eine Positionszuordnung vernehmlich beeinträchtigt.

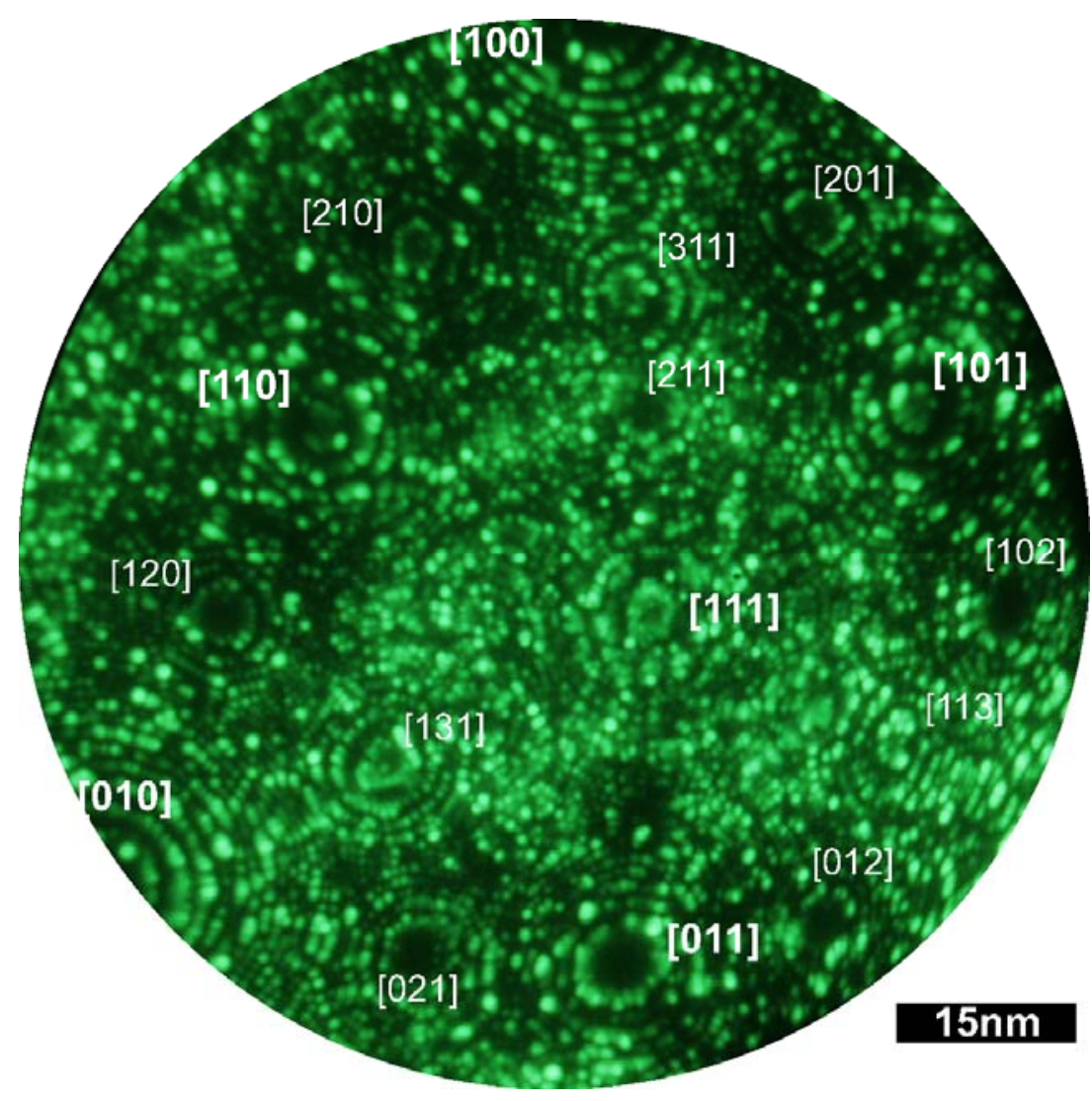

Abbildung 4.2: FIM-Bild von $\mathrm{Fe}_{3} \mathrm{Al}-\mathrm{V}$ bei $35 \mathrm{~K}$ und $11,2 \mathrm{kV}$.

Pole sind indiziert, Hauptrichtungen hervorgehoben.

Folglich sind die FIM-Bilder zur Bestimmung der Platzbesetzung nicht geeignet. Wie aber bereits bemerkt, dienen sie zu einer Überprüfung der Probenqualität und erlauben eine eindeutige Orientierung der Probe und damit der Messrichtung von APT-Messungen.

\subsection{APT-Messungen an $\mathrm{Fe}_{3} \mathrm{Al}$}

Alle APT-Messungen an massivem, geordnetem Material (Kapitel 4 und Kapitel 5) wurden an der Atomsonde am IMP in Göttingen durchgeführt. Zur Anwendung kam eine Spannungsanregung mit einem Puls-Grundspannungs-Verhältnis von $18 \%$. Die Messtemperatur lag bei $30 \mathrm{~K}$, bei den titanhaltigen Proben bei 70K.

Zunächst soll an dieser Stelle die binäre $\mathrm{Fe}_{3} \mathrm{Al}$-Legierung untersucht werden. Die APT-Messung einer $<110>$-orientierten Probe ist in Abbildung 4.3 dargestellt. Deutlich sind die zur Seite ausgerichteten Kristallebenen zu erkennen. Die leichte Schrägstellung rührt daher, dass die Probe in der Atomsonde nicht vollkommen ideal orientiert war (wie in 4.1 beschrieben). Im oberen Bereich des gezeigten Volumens ist die Ebenenauflösung geringer, was ab einem gewissen Abstand vom Zentrum stets der Fall ist. Ein eindimensionales Dichteprofil ist aufgrund der statistischen Schwankungen nur bedingt sinnvoll, stattdessen wurden an den untersuchten Volumina AtomVicinity-Untersuchungen durchgeführt (siehe 3.1.2). Ihr Ergebnis ist in Abbildung 2.1 dargestellt. Dafür wird nicht das gesamte Messvolumen verwendet, sondern nur die Bereiche, bei denen man bereits die höchste Ebenenauflösung erkennen 
kann. In dieser Arbeit werden bei dieser Analyse nur Aluminiumatome als jeweiliges Referenzatom benutzt und Umgebungsatome mit einem Abstand geringer als 7Å für alle drei Koordinaten. Das Resultat ist also ein würfelförmiges Volumen von $14 \AA ̊$ Kantenlänge. Wie Abbildung 4.4 verdeutlicht, sind die Ebenen nach dieser Berechnung klarer zu erkennen als in der ursprünglichen Messung. Um ein Dichteprofil senkrecht zu den Ebenen anzufertigen, muss das Volumen noch orientiert werden, um die leichte Schrägstellung der Ebenen aus Abbildung 4.3 zu kompensieren.

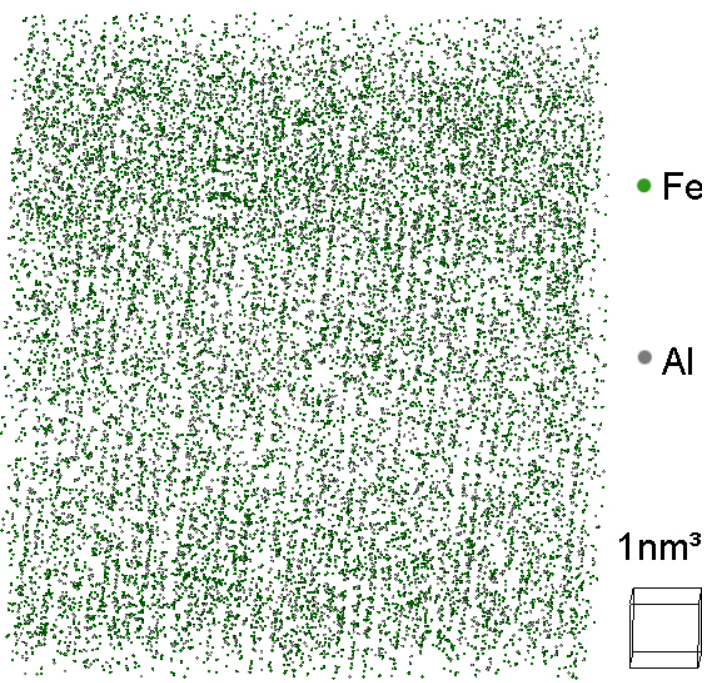

Abbildung 4.3: Ausschnitt aus einem mit der APT gemessenen Volumen

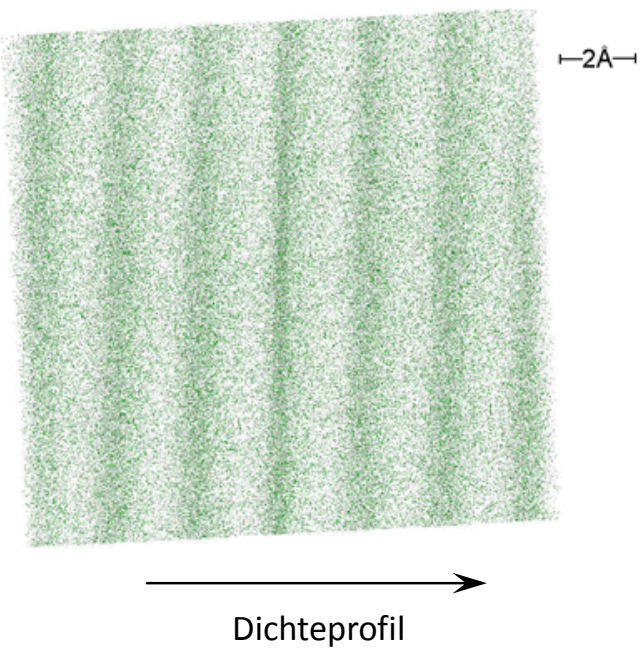

Abbildung 4.4: Ergebnis der AtomVicinity-Analyse. Volumen rotiert für ein Dichteprofil in Richtung der Ebenen.

Um nun diese Dichteprofile für verschiedene Kristallrichtungen zu analysieren, ist es sinnvoll, zunächst einen Blick auf die zu erwartenden Profile zu werfen (siehe dazu Abbildung 4.5). Am relevantesten sind diejenigen der drei Hauptrichtungen $\langle 100\rangle,\langle 110\rangle$ und $\langle 111\rangle$; dabei sind für die betrachtete Struktur alle Richtungen des jeweiligen Typs gleichwertig. Dreht man die Einheitszelle entsprechend, so sieht man, dass in der <110>-Richtung die Ebenen den größten Abstand haben und jede Ebene gleich ist, d.h. zu gleichen Anteilen aus den drei Kristallplätzen besteht. Die $<100>-$ Richtung zeigt zwei verschieden aufgebaute, alternierende Ebenen: eine reine Eisenebene mit den $\alpha$-Plätzen und eine gemischte Ebene mit den $\beta$ - und $\gamma$-Plätzen. Die <111>-Richtung zeigt als einzige vollkommen getrennte, reine Aluminiumebenen ( $\gamma$-Plätze). Dazwischen befinden sich drei Eisenebenen mit jeweils $\alpha-/ \beta$ - $/ \alpha$-Plätzen. Damit ist diese Orientierung prinzipiell am aussagekräftigsten, der geringste Ebenenabstand von $0,85 \AA$ erfordert aber auch die höchste Auflösung.

Diese Ebenenabfolgen können in schematischen Dichteprofilen dargestellt werden, wie sie in Abbildung 4.5 jeweils unterhalb der entsprechend orientierten Kristallstruktur zu sehen sind. Darin sind neben den Ebenenpositionen und -abständen auch die relativen Intensitäten der Elemente und die enthaltenen Kristallplätze abzulesen (wie oben beschrieben). 

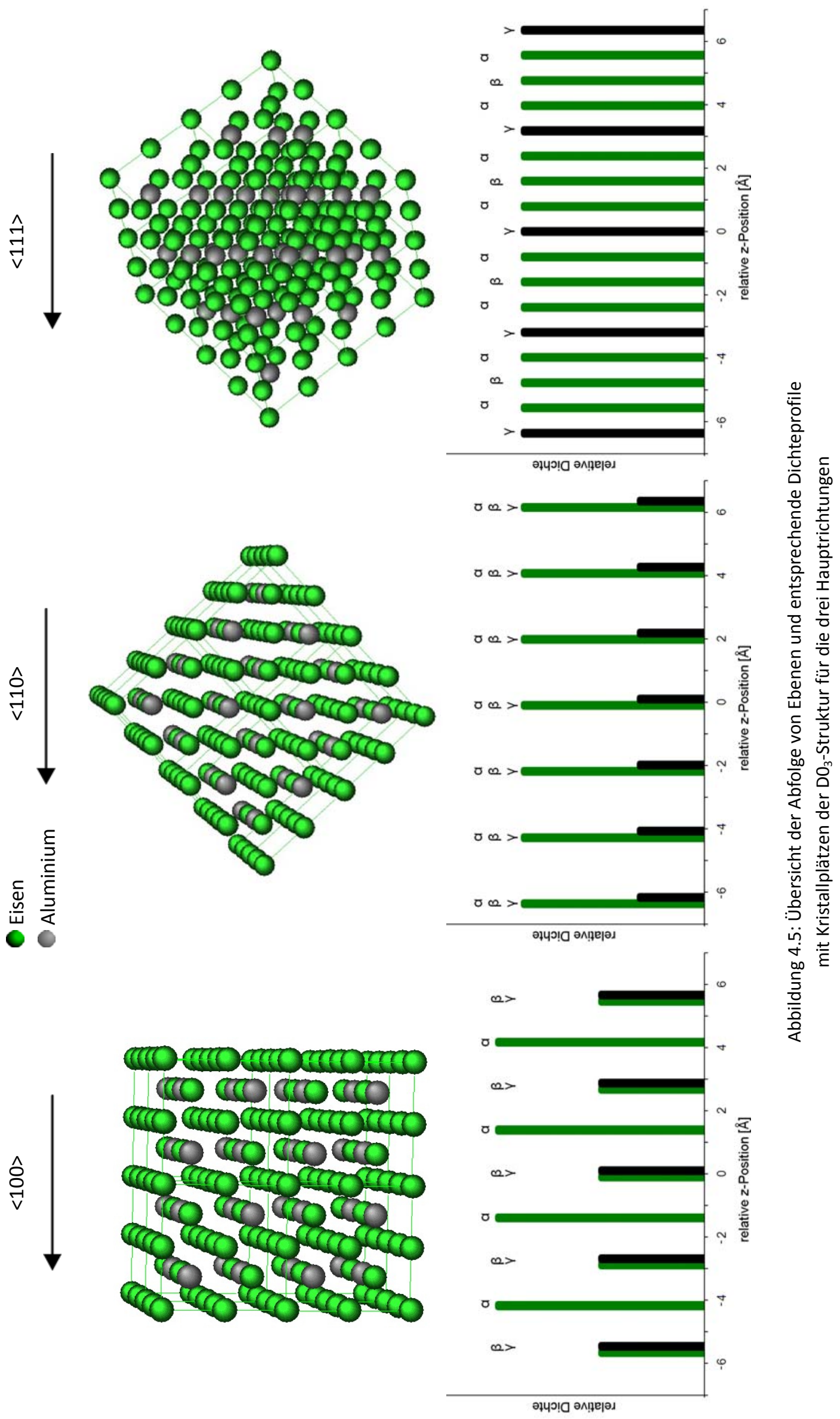
Mit Kenntnis dieser theoretischen Dichteprofile sollen nun die Dichteprofile von APT-Messungen bzw. deren AtomVicinity-Analysen betrachtet werden. Entsprechende Ergebnisse von Messungen an $\mathrm{Fe}_{3} \mathrm{Al}$ für die drei Hauptrichtungen sind in Abbildung 4.6 dargestellt.
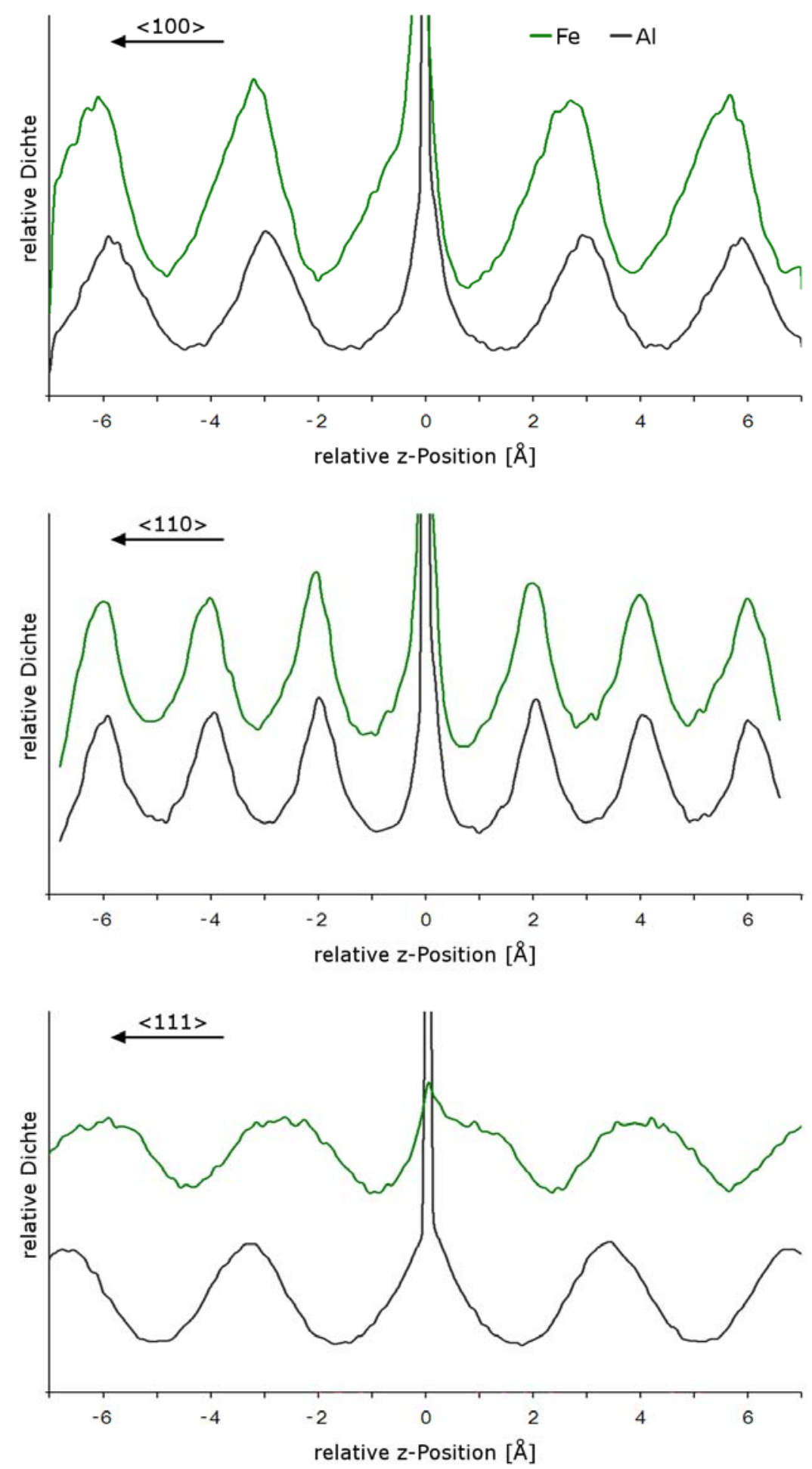

Abbildung 4.6: AtomVicinity-Dichteprofile von APT-Messungen von $\mathrm{Fe}_{3} \mathrm{Al}$

Zunächst fällt der Al-Peak in der Mitte eines jeden Profils aus Abbildung 4.6 auf. Dieser ist dadurch zu erklären, dass man die durchschnittliche Umgebung eines Aluminiumatoms betrachtet, d.h. dass in dessen Zentrum die Antreffwahrscheinlichkeit eines Al-Atoms bei 1 liegt. Die umliegenden Atome zeigen hingegen die (durchschnittliche) Antreffwahrscheinlichkeit, die der Detektionsrate der Atomsonde 
entspricht, also etwa 0,5. Des Weiteren ist die Position der Zentrumsatome festgesetzt und nur in den relativen Positionen der anderen Atome machen sich die Messungenauigkeiten bemerkbar. Daher sind die Peaks, die das Zentrumsatom beinhalten, schärfer (siehe auch 8.1.1).

Trotz dessen und wegen der frei skalierbaren Normierung (siehe 3.1.2) kann man diese Profile aber mit den theoretischen Dichteprofilen vergleichen - unter Beachtung der genannten besonderen Gegebenheiten des mittleren Peaks.

Erst einmal ist für die $<110>-$ Richtung zu bemerken, dass die Ebenen wie zu erwarten ausgemacht werden können: sie zeigen jeweils eine gemischte Komposition mit dem erwarteten Abstand.

In der <111>-Richtung sind die Aluminium-Peaks ebenfalls im theoretisch bestimmten Abstand der Aluminiumebenen auszumachen. Die drei dazwischen liegenden Eisenebenen können hingegen nicht getrennt aufgelöst werden, die Messgenauigkeit der APT ist nicht ausreichend. Der daraus resultierende, breite Eisenpeak ist zudem nach links relativ zur erwarteten Position verschoben. Ein ähnliches Verhalten kann für die <100>-Richtung beobachtet werden: Die Aluminium-Peaks sind im Abstand entsprechend der Aluminiumebenen zu erkennen. Das Eisen hingegen ist auch hier nach links verschoben, der Peak der reinen Eisenebene ist nach links verlagert bis zum Aluminium-Peak, der kleinere Eisen-Peak der gemischten Ebene ist ebenfalls etwas nach links umpositioniert, beide Eisen-Peaks überlappen sich und bilden einen einzigen, etwas asymmetrischen Peak.

Die Eisenatome scheinen also relativ zum Aluminium nach links verschoben, selbst bei der $\langle 110\rangle-$ Richtung kann ein kleiner Unterschied in der Peakposition ausgemacht werden, wenngleich deutlich geringer als in den anderen Richtungen. Innerhalb dieser Grafiken bedeutet nach links verschoben, ein geringerer Wert der z-Komponente, also verschoben in Richtung der Probenspitze.

Wie kann nun erklärt werden, dass die Eisenatome relativ zu den Aluminiumatomen in unterschiedlichem Maße eine zu gering berechnete z-Komponente haben? Es handelt sich dabei um einen Effekt, der schon in anderen APT-Studien bemerkt wurde und der auf unterschiedlichen Feldverdampfungsfeldstärken beruht [58, 78, 79]. Die Überlegungen zur Rekonstruktion der Atome aus den Detektorsignalen (siehe 3.1.1) basieren auf der Annahme, dass alle Atome die gleiche Feldverdampfungsfeldstärke haben. Die Halbkugel, auf die die Atome projiziert werden, stellt damit eine Äquipotentialfläche der Verdampfungsfeldstärke dar. Wenn es aber nun Atome gibt, die schon bei geringerer Feldstärke evaporieren, tun sie dies ggf., wenn sie noch unterhalb der Halbkugel sitzen (vergl. Abbildung 4.7). Für den Rekonstruktionsalgorithmus verdampfen sie also zu früh. Genau dies ist hier der Fall: Die Eisenatome besitzen eine geringere Verdampfungsfeldstärke (siehe auch FIM-Aufnahmen in 4.1), verdampfen etwas eher als durch die geometrischen Verhältnisse erwartet. Dieses Verhalten der verfrühten Evaporation wurde auch schon von FROMMEYER et al. [58] anhand von FIM-Aufnahmen beschrieben, GE et al. erklärten diesen Effekt schematisch anhand von Bindungsenergien [79].

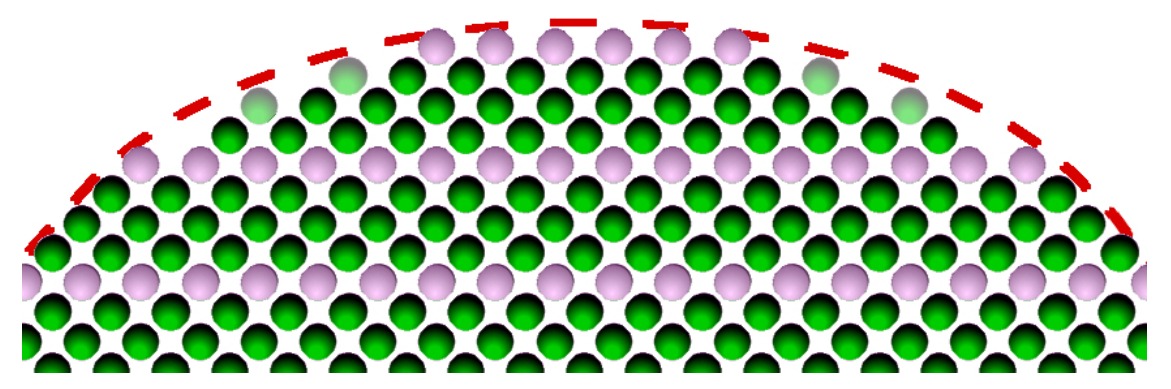

Abbildung 4.7: Schematische APT-Spitze.

_ globale Spitzenform $\bigcirc \mathrm{Al}$ - Fe $\mathrm{Fe}$, das als nächstes evaporiert, obwohl es exponiertere Aluminiumatome gibt 
Durch dieses Phänomen kann es nun passieren, dass zwei Atome aus unterschiedlichen (untereinander liegenden) Ebenen quasi direkt nacheinander (bzw. relativ schnell) evaporieren und in etwa an der gleichen Stelle am Detektor ankommen. Erfolgt dann aus den Detektorsignalen die Rekonstruktion der Atompositionen, werden die Atome - nach den Formeln aus 3.1.1 - auf etwa gleiche Positionen berechnet. Dadurch wird dem Atom aus der unteren Ebene, nämlich dem mit der geringeren Evaporationsfeldstärke, eine zu geringe z-Komponente zugeordnet. Die $x$ - und y-Position können durch das zu frühe Evaporieren ebenfalls beeinflusst werden, da sich auch Projektionsverhältnisse geringfügig ändern können, im Wesentlichen durch einen anderen Apexradius. Diese Ungenauigkeit ist aber deutlich geringer als die der z-Komponente und ist kleiner als die allgemeine Messgenauigkeit der $x$ - und yPosition $^{\text {iv }}$. Weitere Überlegungen zu diesem Effekt und zusätzliche Auswirkungen werden in 4.5 beschrieben.

Nach den Dichteprofilen aus Abbildung 4.6 ist die Verschiebung der Eisen-Peaks messrichtungsabhängig. Um dies zu erklären, sind die Ebenen zu betrachten, die senkrecht zur Messrichtung stehen und deren Atome sukzessiv während der Messung von außen nach innen evaporieren.

In <111>-Richtung gibt es reine Aluminiumebenen. Beginnt eine davon zu verdampfen, liegen darunter drei reine Eisenebenen, die nach dem oben beschriebenen Effekt - relativ zum Aluminium - zu früh evaporieren. Dadurch wird das Eisen kollektiv mit einer zu geringen z-Komponente und damit im Dichteprofil zu weit links dargestellt.

Hingegen ist das in $<110>-$ Richtung nicht der Fall, in der es nur gemischte Ebenen gibt. Diese haben auch die größte Ebenenflächendichte und den größten Abstand. Verdampft hier ein Aluminiumatom, gibt es Eisenatome, die dadurch frei liegen, sowohl in der gleichen Ebene als auch in der darunter. In der unterhalb liegenden Ebene ist die Feldstärke aber aufgrund des relativ großen Abstandes deutlich geringer. Es evaporieren also erst die der ersten Ebene, dann die aus der darunter. Dadurch entspricht die Verdampfungsreihenfolge aber der geometrisch zu erwartenden, wodurch es auch kaum zu einer falschen Berechnung der Atompositionen kommt.

In $<100>$-Richtung liegt in etwa ein gemischter Fall vor: Auch die reine Eisenebene erfährt eine zu frühe Evaporation, es gelten die Überlegungen zur <111>-Richtung. Die Eisenatome der gemischten Ebene verhalten sich etwa so wie in <110>-Richtung, da diese Ebene aber eine geringere Flächendichte und damit auch weniger Bindung zu Nachbaratomen hat als in der $\langle 110\rangle-$ Orientierung, ist eine etwas zu frühe Evaporation möglich und folglich auch eine mäßige Fehlpositionierung.

Solche Rekonstruktionseffekte sind bekannt und es gibt Ansätze, sie zu minimieren. Beispielsweise können Aluminium und Eisen mit unterschiedlichen Verdampfungsfeldstärken rekonstruiert und damit auf zwei unterschiedliche Halbkugeln zurückprojiziert werden [62]. Allerdings stößt man für $\mathrm{Fe}_{3} \mathrm{Al}$ dabei auf einige Probleme: Erstens gibt es prinzipiell zwei verschiedene Eisenplätze ( $\alpha$ und $\beta$ ), die sich unterschiedlich verhalten können, die aber durch das Detektorsignal nicht unterschieden werden können. Zweitens bedarf es für eine genaue Berechnung der Kenntnis der Verdampfungsfeldstärken jedes einzelnen Elements. Man kennt diese jeweils für die reinen Phasen $\left(19 \mathrm{~V} / \mathrm{nm}\right.$ für $\mathrm{Al}^{+}, 35 \mathrm{~V} / \mathrm{nm}$ für $\mathrm{Al}^{++}$und $33 \mathrm{~V} / \mathrm{nm}-35 \mathrm{~V} / \mathrm{nm}$ für $\mathrm{Fe}^{++}$[47]), für die Legierung sind diese aber nicht bekannt. Augenscheinlich sind sie - aufgrund anderer Bindungsverhältnisse - anders, denn Aluminium weist hier die höhere Verdampfungsfeldstärke auf, wie die Ergebnisse aus 4.1 und 4.2, sowie [58, 77, 79] zeigen. Zusätzlich muss die Messrichtungsabhängigkeit beachtet werden. Das bedeutet effektiv, dass die Bindungsverhältnisse eines Elements in verschiedenen Richtungen unterschiedlich sind und damit auch die Feldverdampfungsfeldstärken. Die Berechnung von Verdampfungsfeldstärken ist dabei nur ungenügend exakt mög-

${ }^{\text {iv }}$ Eine Abschätzung zum Einfluss auf die $x$ - und y-Position ist im Anhang zu finden (C.2). 
lich, da über den genauen Evaporationsprozess noch zu wenige Erkenntnisse gewonnen wurden (vgl. 3.3.2). Die Abweichungen von gemessenen und berechneten Verdampfungsfeldstärken nach gängigen Modellen liegt teilweise bei über 35\% [47].

Diese Effekte können also direkt kaum umgangen werden. Jedoch erschweren sie die Studien über Ordnung und Platzbesetzung, insbesondere bei ternärem $\mathrm{Fe}_{3} \mathrm{Al}$, da die gemessene Ebeneabfolge ggf. nicht der Kristallstruktur entspricht, sondern das Ergebnis von diesen Rekonstruktionseffekten sein kann. Beispielsweise kann eine Überlagerung eines Aluminium- und Chrom-Peaks in einem AtomVicinity-Dichteprofil bedeuten, dass diese Elemente in einer Ebene sitzen. Es kann aber auch nicht ausgeschlossen werden, dass sie in verschiedenen Ebenen positioniert sind und es aufgrund von verfrühter Evaporation zu Peakverschiebungen im Dichteprofil und damit zu einer Überlagerung kommt.

Wie in Kapitel 5 gezeigt wird, können solche Effekte teilweise durch die systematische Analyse der Dichteprofile entlang der drei Hauptrichtungen abgeschätzt werden. Für exaktere Aussagen - insbesondere bei der genauen Bestimmung von Ordnungsgraden - ist der Einsatz von Simulationen von APT-Messungen sinnvoll. Indem von verschiedenen Kristallordnungen mit unterschiedlicher Platzbesetzung der ternären Zusätze APT-Messungen simuliert und diese mit den gemessenen APT-Ergebnissen verglichen werden, können trotz der geschilderten Rekonstruktionseffekte Aussagen über die Kristallstruktur und -ordnung getroffen werden. 


\subsection{Simulation von APT-Messungen an $\mathrm{Fe}_{3} \mathrm{Al}$}

\subsubsection{Optimierung der Parameter}

Zunächst werden an dieser Stelle Messungen der binären Legierungen simuliert, um grundlegende Simulationsparameter zu eichen. Die dafür benötigten Paarpotentiale sind in der Literatur zu finden, gerade für die reinen Elemente gibt es eine Fülle von Angaben. Der genaue Verlauf der Potentiale unterscheidet sich dabei jedoch. So werden für das Fe-Fe-Potential Energieminima zwischen $0,19 \mathrm{eV}$ und $0,39 \mathrm{eV}$ angegeben [72, 80-87]. Die Position des Minimums ist hingegen ähnlicher, da es sich direkt am NN-Abstand der reinen Phase orientiert. Auch für Aluminium gibt es verschiedene Angaben, die alle einen ausgedehnteren Potentialverlauf mit einem geringer ausgeprägten Energieminimum als das FeFe-Potential [81-83, 88, 89] aufzeigen. Das Fe-Al-Potential wird in verschiedenen Quellen beschrieben [81-83], die Position und Tiefe des Energieminimums ist dabei jeweils gleich oder geringfügig größer als diejenigen des Fe-Fe-Potentials.

Die für die Simulation verwendeten Paarpotentiale wurden demnach im Bereich dieser Literaturwerte derart angepasst, dass es zu einer größtmöglichen Übereinstimmung zwischen den Simulationen und den APTMessungen kommt. Sie sind nebenstehend in Abbildung 4.8 dargestellt.

Die Elektronendichtefaktoren $\rho_{0}$ sind aus [72] entnommen bzw. nach Formel 3.15 bestimmt. Diese und die zur Berechnung benötigten, materialspezifischen Werte sind in Tabelle B.1 im Anhang verzeichnet.

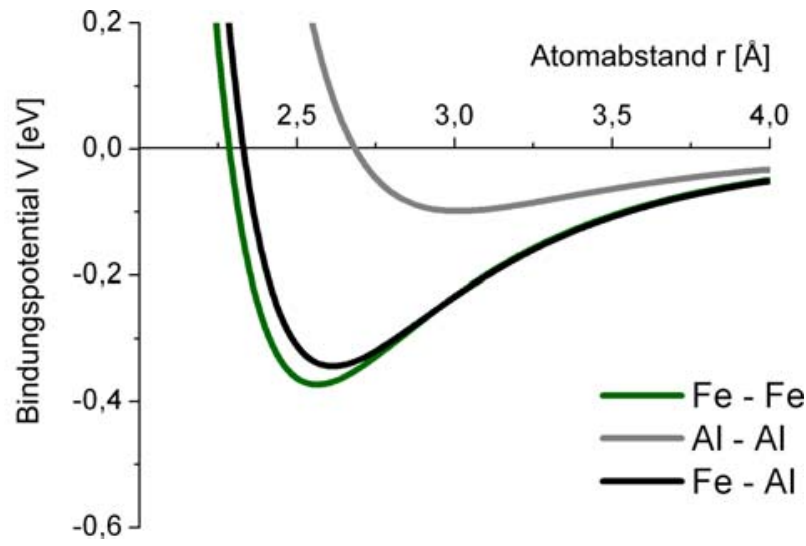

Abbildung 4.8: Paarpotentiale der binären Legierung

Ebenso müssen der Feldenergiefaktor $\mathrm{D}$ sowie der lonisierungsenergiekoeffizient $\mathrm{K}$ bestimmt werden. Diese wurden ebenfalls durch Anpassen von Messung und Simulation ermittelt, im Rahmen dieser Arbeit wurden die Werte $\mathrm{D}=0,045 \mathrm{eV} / \mathrm{A}^{-2}$ sowie $\mathrm{K}=0,35$ verwendet. Des Weiteren wurden die Atompositionen der simulierten Volumina nach dem Zufallsprinzip leicht geändert, um Messungenauigkeiten ebenfalls zu beachten. Die $x$-, $y$ - und z-Positionen wurden dazu unabhängig voneinander gaussförmig „verschmiert“, der entsprechende $\sigma$-Wert wurde individuell jeder Messung angepasst. Für die AtomVicinity-Dichteprofile ist dabei nur die z-Verschmierung relevant.

\subsubsection{Analyse der Simulationen}

Von den simulierten APT-Daten wurden AtomVicinity-Dichteprofile angefertigt (Abbildung 4.9), um das Simulationsergebnis genau zu analysieren und mit den Messungen (Abbildung 4.4) zu vergleichen zu können.

Insgesamt zeigen die simulierten Profile eine große Übereinstimmung mit den gemessenen Profilen: In $<110>$-Richtung sind die Ebenen zu erkennen, bei denen Eisen und Aluminium eine nur geringe relative Peakverschiebung von ca. $0,2 \AA$ aufzeigen. In $<111>$-Orientierung sind die Eisen-Peaks zu einem Peak fusioniert, der ebenfalls nach links verschoben ist. Auch in $<100>$-Richtung ist diese Verschiebung des Eisens zu erkennen. Damit ist der Effekt der verfrühten Evaporation, der in den Messungen festgestellt wurde, richtig simuliert worden. 

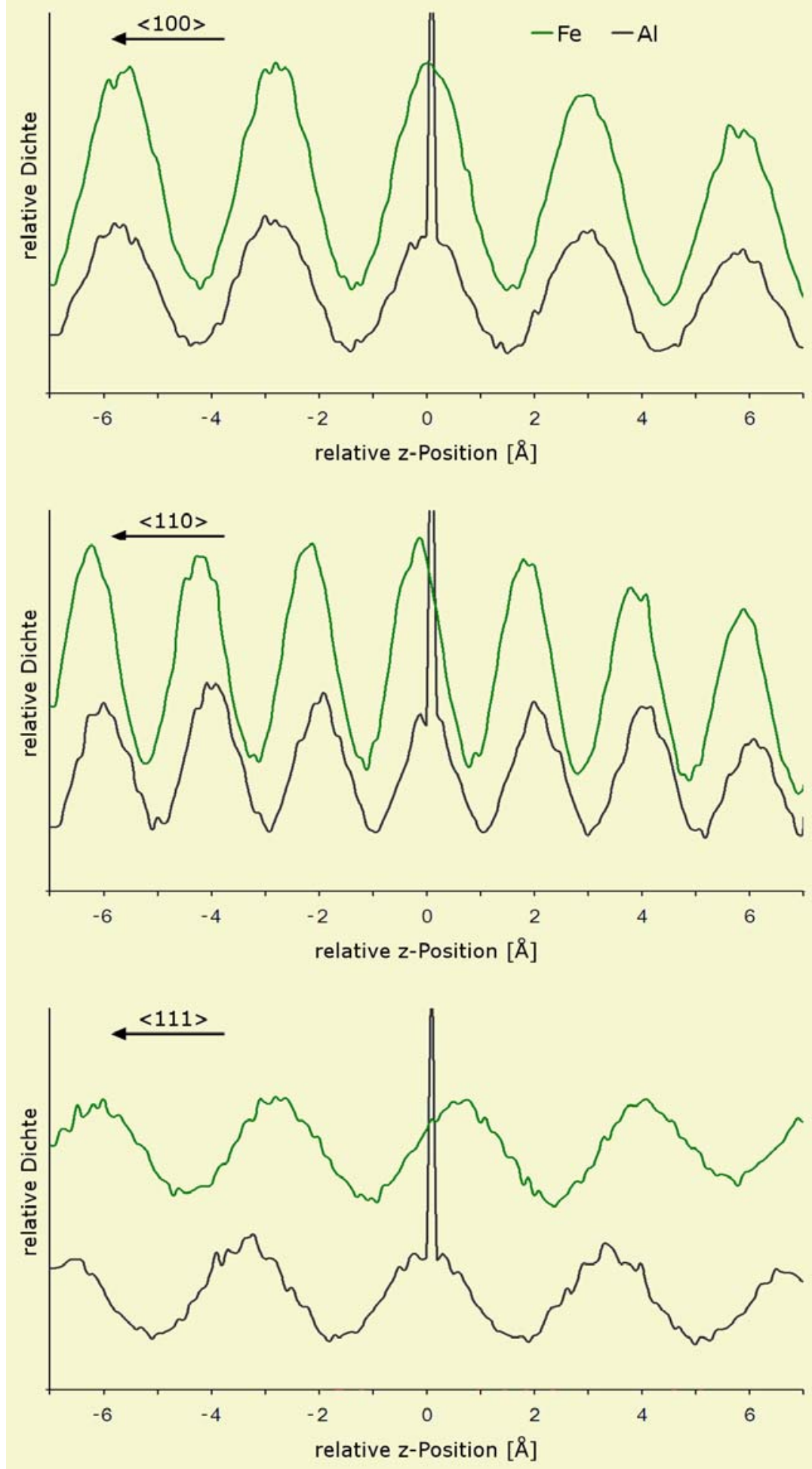

Abbildung 4.9: AtomVicinity-Dichteprofile einer simulierten APT-Messung von $\mathrm{Fe}_{3} \mathrm{Al}^{\vee}$

Des Weiteren ist gezeigt, dass Aluminium im $\mathrm{Fe}_{3} \mathrm{Al}$ in der Tat die höhere Feldverdampfungsfeldstärke besitzt, so wie es die FIM-Bilder veranschaulichen. Der Grund dafür ist, dass Aluminium in $\mathrm{Fe}_{3} \mathrm{Al}$ die höhere Oberflächenbindungsenergie aufweist. Dies entspricht den Abschätzung von GE et al [79],

Zur schnelleren visuellen Unterscheidung von gemessenen und simulierten AtomVicinity-Dichteprofilen sind die der Simulationen hellgelb unterlegt. 
wenngleich sie eine einfachere Berechnungsmethode nur mit Paarpotentialen wählten. Zunächst ist die höhere Bindungsenergie von Aluminium insofern nicht zu erwarten, als dass es eine geringere Sublimationsenergie besitzt als Eisen. Es kann aber durch die Berechnung von $E_{B}$ anhand von Formel 3.13 erklärt werden: Bei Aluminium sind die Paarpotentiale in ihrer Stärke geringer, dafür zeigt Aluminium eine höhere Bindung durch den zweiten Term aus Formel 3.13. Dieser skaliert aber mit der Wurzel der Anzahl der umliegenden Atome, während die Paarpotentiale proportional dazu sind. Verliert ein Aluminiumatom also während der Feldverdampfung der Spitze einige Umgebungsatome, so sinkt seine Bindung weniger stark als bei einem Eisenatom. Ergänzend unterstützt auch die Ionisierungsenergie diesen Effekt.

Anzumerken ist noch, dass die Positionen der Peaks weitestgehend richtig simuliert werden können, die Peakformen aber teilweise erkennbare Unterschiede aufzeigen. Dies kann an bestimmten Effekten der Feldevaporation liegen, die in den Simulationen nicht eingebracht wurden. Dieser Aspekt soll in 8.2.2 genauer diskutiert werden. Dementsprechend soll aber in den weiteren Analysen der Simulationen insbesondere Wert auf eine richtige Position der Peaks gelegt werden.

\subsection{Ordnungsgrade}

Die Simulationen bieten weiterhin die Möglichkeit, den Ordnungsgrad der $\mathrm{DO}_{3}$-Ordnung zu ermitteln. Insbesondere die aufgrund ihrer Ebenenabfolge aussagekräftige <111>-Richtung ist dafür dienlich. Dazu folgende Überlegung: Die Peaks zeigen eine gewisse Unschärfe, teilweise kann man bei den Graphen sogar von einem Hintergrundsignal sprechen. Diese Effekte können aus der Messungenauigkeit entstehen, sie können aber auch Folge von fehlpositionierten Atomen im Probenmaterial sein. Zu beachten ist dabei, dass sich bei diesen Atomen die Bindungsverhältnisse ändern und dadurch auch ihr Evaporationsverhalten. Daher ist bei ihnen eine veränderte Peakverschiebung möglich, die Beachtung finden muss. Eine Aussage über Ordnung allein aus den Messungen ist a priori daher nicht möglich, Simulationen hingegen können Angaben liefern, die diesen Effekt berücksichtigen.

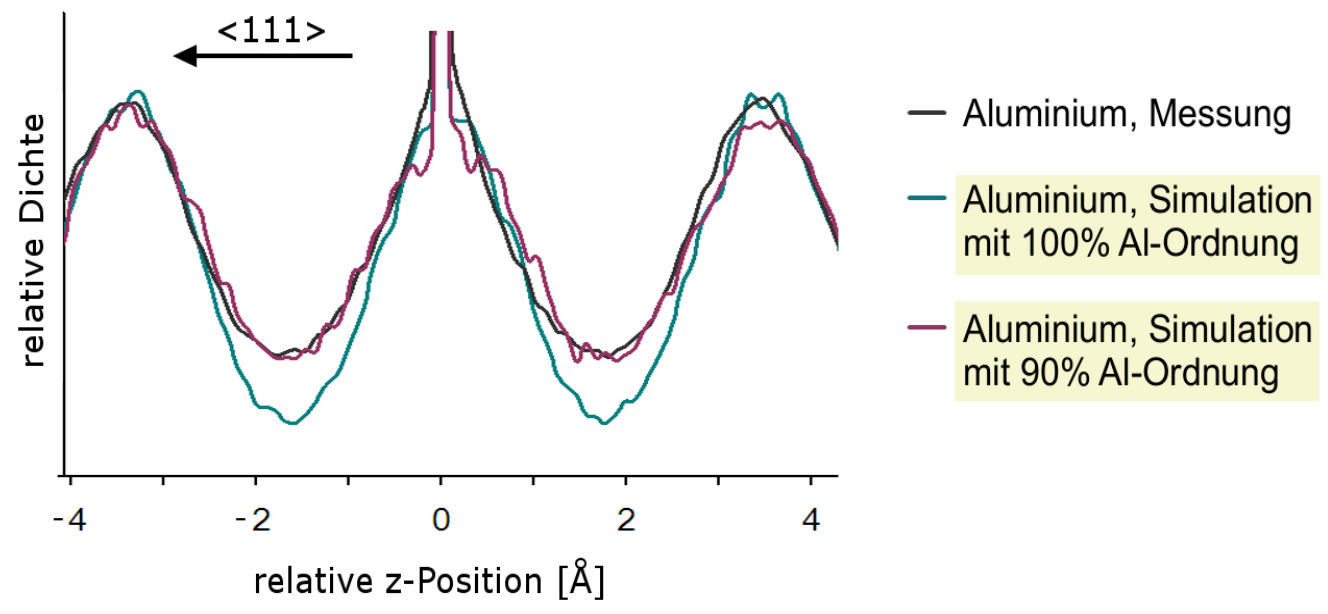

Abbildung 4.10: AtomVicinity-Dichteprofile von Aluminium:

Messung und Simulationen mit verschiedenen Ordnungsgraden

Um nun den genauen Verlauf der Graphen zu simulieren, müssen Ordnungsgrad und Messunschärfe angepasst werden. Bei letzterem wird eine einheitliche Gaussverschmierung (siehe 3.3.1) für alle Elemente angenommen.

Praktisch soll dies an den <111>-Dichteprofilen des Aluminiums erläutert werden, siehe Abbildung 4.10. Zunächst wird eine Simulationen für eine perfekt geordnete $\mathrm{Fe}_{3} \mathrm{Al}$-Kristallstruktur durchgeführt und die Messunschärfe anhand des Verlaufs des Eisengraphs (in der Abbildung nicht dargestellt) ange- 
passt. Der Aluminium-Peak ist dann jedoch noch zu schmal, zwischen den Peaks zeigen Messung und Simulation eine starke Diskrepanz. Verändert man den Ordnungsgrad, d.h. verringert man den Anteil von Al auf $\gamma$-Plätzen und erhöht inn zufällig verteilt auf $\alpha$ - oder $\beta$-Plätzen, kann diese Diskrepanz aufgehoben werden. Insgesamt müssen also sowohl Gaussverschmierung als auch Ordnungsgrad derart angepasst werden, dass für alle Elemente sowohl das Peak-Rauschsignal-Verhältnis möglichst gut übereinstimmt, als auch die Peakform. Letztere kann dabei im Wesentlichen nur auf das Verhältnis von Breite und Höhe angepasst werden, da das genaue Profil - wie oben beschrieben - nur ungenau simuliert werden kann.

Durch den Vergleich mit der Simulation können ebenfalls Unschärfen, die auf das Rekonstruktionsprinzip zurückgehen, beachtet werden. Diese entstehen, da der Ablauf der Rekonstruktion diskret, also in festen Schrittweiten erfolgt. Zu erkennen sind sie, indem eine Simulation perfekter Ordnung ohne Messunschärfe simuliert wird, auch hier ergeben sich Peakbreiten (FWHM) von ca. 0,2Å.

Insgesamt kann mit diesem Prinzip eine Aluminiumordnung von $90 \% \pm 5 \%$ in der binären Legierung ermittelt werden.

\subsection{Elementkonzentrationen}

Aus den Messdaten der Atomsonde lassen sich die Konzentrationen der enthaltenen Elemente berechnen. In Tabelle 4.2 ist die gemittelte Konzentration insgesamt und aufgeteilt nach Messrichtung angegeben. Es sind hier die relativen Konzentrationen zu den Nominalkonzentrationen genannt, da letztere - abhängig von den ternären Zusätzen - für Eisen variieren.

\begin{tabular}{|c|c|c|c|c|c|c|}
\hline \multirow{3}{*}{ Element } & \multirow{3}{*}{$\begin{array}{c}\text { nominelle } \\
\text { Konzentration }\end{array}$} & \multicolumn{4}{|c|}{ APT-Messungen } & \multirow{3}{*}{$\begin{array}{c}\text { EDX- } \\
\text { Messungen }\end{array}$} \\
\hline & & & Messrichtun & & g & \\
\hline & & $<100>$ & $<110>$ & $<111>$ & gesdmi & \\
\hline \multirow[t]{2}{*}{ Aluminium } & $26 a t \%$ & $+2,7$ & $+7,1$ & $+3,0$ & $+4,0$ & $-2,6$ \\
\hline & & $\pm 1,4$ at $\%$ & $\pm 1,8 \mathrm{at} \%$ & $\pm 1,1$ at $\%$ & $\pm 2,3 a t \%$ & $\pm 2,0$ at $\%$ \\
\hline \multirow[t]{2}{*}{ Eisen } & 69at\%/ & $-4,5$ & $-8,4$ & $-4,1$ & $-5,4$ & $+2,4 \%$ \\
\hline & $\begin{array}{l}\text { 70at\%/ } \\
\text { 74at\% }\end{array}$ & $\pm 2,0$ at $\%$ & $\pm 1,9 a t \%$ & $\pm 2,5$ at $\%$ & $\pm 2,7$ at $\%$ & $\pm 1,8$ at $\%$ \\
\hline
\end{tabular}

Tabelle 4.2: Gemessene Konzentrationen mit der APT relativ zur Nominalkonzentration

Es ist deutlich zu erkennen, dass Aluminium eine erhöhte Detektionsrate aufweist, Eisen hingegen eine verminderte. Das Auftreten einer ungenau gemessenen Konzentration mit der APT ist ein bekannter Effekt [50, 78], der auf unterschiedlichen Feldverdampfungsfeldstärken beruht. Ist diese bei einem Element höher (hier Aluminium), so bestimmt dieses die Evaporationsrate, wie es in 4.1 beschrieben wurde. Das Eisen zeigt hingegen den Effekt der verfrühten Evaporation. Diese Tatsachen können dazu führen, dass es zu einer korrelierten Evaporation kommt [50]. Das bedeutet, dass erst wenn ein Aluminiumatom verdampft ist, daneben- oder dahinterliegende Eisenatome evaporieren können, auch wenn deren Verdampfungsfeldstärke bereits erreicht war. Sie evaporieren dann unmittelbar nach dem Aluminiumatom, zeigen dann allerdings eine geringere Detektionsrate durch den Detektor. Dafür gibt es mehrere Gründe:

- Da der Multianodendetektor nur eine begrenzte Anzahl an Ereignissen pro Spannungspuls verarbeiten kann, ist bei den nachfolgend evaporierenden Atomen die Wahrscheinlichkeit höher, nicht mehr registriert zu werden. Diese Anzahl liegt je nach Bauart und Modus des Detektors im Allge- 
meinen zwischen drei und acht, bei der verwendeten Atomsonde für diese Messungen bei drei bis vier.

- Die an den Mikrokanalplatten erzeugten Ladungswolken können aufgrund einer gewissen benötigten Erholzeit der Platten beim zweiten Atom pro Spannungspuls zu gering sein, um ein verwertbares Detektorsignal auszulösen. Der Flugzeitunterschied von zwei Atomen beträgt etwa eine Mikrosekunde, auch die Erholzeit liegt im Bereich von Mikrosekunden [90]. Relevant wird dieser Effekt, wenn - wie bei der korrelierten Evaporation - in etwa die gleiche Stelle an den Platten getroffen wird.

- Das Eisenatom evaporiert zwischen den Spannungspulsen. Da die Feldverdampfungsfeldstärke höher als benötigt ist, kann nicht ausgeschlossen werden, dass auch Evaporation stattfindet, wenn der Spannungspuls nicht überlagert ist und die Grundfeldstärke ausreicht. Eine Flugzeitmessung und Massenbestimmung kann dann nicht durchgeführt werden.

Das Auftreten dieser Effekt bedeutet also, dass die Wahrscheinlichkeit, ein verwertbares Detektorsignal zu erzeugen, bei einem Atom mit der geringeren Verdampfungsfeldstärke kleiner ist, die gemessene Konzentration relativ zu der anderen Elementsorte sinkt.

Ein deutlicher Beleg für dieses Verhalten ist die Anzahl der Spannungspulse, die zwischen dem letzten Detektorsignal und einem neuen Evaporationsereignis gegeben wurden. Die Quote an Eisenatomen, die unmittelbar nach einem anderen Atom evaporierten (also während desselben Spannungspulses) liegt bei Eisen bei ca. 12\% aller Atome, bei Aluminium ist sie hingegen nur etwa 1,5\%. Dazu kommt, dass $9 \%$ aller Eisenatome direkt nach einem Aluminiumatom evaporieren und die Hälfte davon auch eine ähnliche Detektorposition wie das Al-Atom haben. Bei letzteren kann man also davon ausgehen, dass Al und Fe nächste Nachbarn waren und korreliert evaporierten. In diesen Zahlen sind nun aber Ereignisse enthalten, bei denen beide Atome erfolgreich detektiert wurden. Rückschlüsse auf die Zahl nicht detektierter Fe-Atome kann man daraus nicht gewinnen und daher die zu gering gemessene Eisenkonzentration nicht quantitativ erklären. Sie zeigen aber, dass die korrelierte Evaporation ein wichtiger Effekt der Feldverdampfung in dieser Legierung ist und bestätigen abermals die Überlegungen zur verfrühten Evaporation aus 4.1.

Wie Tabelle 4.2 verdeutlicht, ist dieser Effekt offenkundig messrichtungsabhängig. Um dies zu erklären, muss man einerseits natürlich beachten, dass die Ebenenanordnung und Atompositionen relativ zur Messrichtung unterschiedlich sind. Andererseits muss man bedenken, inwiefern sich der oben geschilderte Effekt der korrelierten Evaporation des Eisens bemerkbar machen kann. Augenscheinlich werden insbesondere solche Eisenatome durch Aluminiumatome zurückgehalten, die nächste Nachbarn zum Aluminium sind, also Eisen auf $\alpha$-Plätzen. Entscheidend ist nun die Tatsache, wo diese nächsten Nachbarn sitzen: In <110>-Richtung liegen sie in der gleichen Ebene wie das Aluminium. Ist also selbiges verdampft, liegen diese Eisenatome frei und unterliegen in etwa dem gleichen elektrischen Feld wie das Aluminium, d.h. in diesem Fall kommt der Effekt der korrelierten Evaporation sehr zum Tragen.

Anders in $<100>$ - oder $<111>$-Richtung. Das $\alpha$-Platz-Eisenatom, das beim Verdampfen eines Aluminiumatoms frei wird, liegt in einer darunterliegenden Ebene. Dort ist aber das elektrische Feld geringer und damit auch die Wahrscheinlichkeit zu evaporieren. Also macht sich in dieser Konstellation der genannte Effekt nicht so stark bemerkbar. Zusätzlich haben die Eisenatome in einer darunterliegenden Ebene auch noch mehr Umgebungsatome als ein freiliegendes Eisenatom in $<110>$-Richtung. Dadurch sind sie stärker gebunden und das elektrische Feld ist auch deswegen nochmals geringer, was beides dem Effekt der korrelierten Evaporation entgegenwirkt. 
Die Konzentrationen der ternären Zusätze sind:

\begin{tabular}{lccc}
\hline Zusatz & $\begin{array}{c}\text { nominelle } \\
\text { Konzentration [at\%] }\end{array}$ & $\begin{array}{c}\text { Konzentration aus } \\
\text { APT-Messungen [at\%] }\end{array}$ & $\begin{array}{c}\text { Konzentration aus } \\
\text { EDX-Messungen [at\%] }\end{array}$ \\
\hline Chrom & 5 & $6,8 \pm 2,8$ & $5,31 \pm 0,17$ \\
Mangan & 5 & $3,5 \pm 1,3$ & $5,49 \pm 0,41$ \\
Vanadium & 4 & $5,7 \pm 1,6$ & $4,03 \pm 0,13$ \\
Titan & 4 & $5,8 \pm 1,2$ & $3,93 \pm 0,39$ \\
\hline
\end{tabular}

Tabelle 4.3: Konzentrationen der ternären Zusätze der massiven Proben

Augenscheinlich gibt es auch bei den Zusätzen deutliche Schwankungen in den durch die APT gemessenen Konzentrationen. Oben genannte Gründe für diese Abweichungen gelten analog natürlich auch für die Additionen, wobei hier einschränkend beachtet werden muss, dass die Platzbesetzung entscheidenden Einfluss hat. Sitzt das ternäre Element auf $\beta$-Plätzen, ist hier eine korrelierte Evaporation mit Aluminium unwahrscheinlicher, da sie nicht (bzw. bei nicht kompletter Ordnung seltener) nächste Nachbarn sind.

Messrichtungsabhängigkeiten bei den Zusätzen konnten nicht festgestellt werden. Allerdings sind pro Zusatz und Messrichtung maximal drei Messungen durchgeführt worden, oftmals sogar weniger, so dass die statistische Signifikanz relativ gering ist.

Die EDX-Messungen im Rahmen der ALCHEMI-Messungen (Kapitel 6) zeigen deutlich genauere Ergebnisse, die ebenfalls jeweils im Bereich der Messgenauigkeit der nominellen Konzentration relativ gut entsprechen. Größere Abweichungen von der nominellen Konzentration zeigen sie bei Aluminium und Eisen. Man kann vermuten, das dies an der Kalibrierung der EDX-Auswertung liegt, welche bei größeren Konzentrationen auch entsprechend größere absolute Fehler ergibt. D.h. dass sich in diesem Fall dieser systematische Fehler stärker auswirkt als der statistische.

\subsection{Geometrischer Einfluss}

Wie in 3.1 geschildert, haben $r_{k}$ und auch der Schaftraduis $r_{s}$ Einfluss auf die rekonstruierten Koordinaten. Daher ist es sinnvoll, dass in der Simulation realistische Werte von $r_{k}$ und $r_{s}$ benutzt werden. Das Problem bei entsprechend großen Volumina ist jedoch, dass die Simulationsdauer im Bereich von einigen Stunden liegt. Damit ist effektives Arbeiten nicht mehr gewährleistet, da zur Optimierung aller Parameter hunderte Simulationen notwendig sind.

Um zu kleineren Volumen überzugehen, müssen $r_{k}$ und $r_{s}$ vermindert werden. Nach Formel 3.17 ist das Verhältnis dieser beiden Radien konstant und wird bestimmt durch die Geometrien des Atomsondenaufbaus. Bei der für alle Messungen aus Kapitel 4 und Kapitel 5 verwendeten Atomsonde gelten die Abmessungen $L=0,45 \mathrm{~m} ; r_{\text {det }}=0,05 \mathrm{~m}$ und $\eta=1,5$. Damit liegt das Verhältnis $r_{s} / r_{k}$ bei etwa $1 / 6$. Typische Werte einer Messung sind $r_{s} \approx 10 \mathrm{~nm}$ und $r_{k} \approx 60 \mathrm{~nm}$, was ebenfalls ein Verhältnis von $\sim 1 / 6$ ergibt und damit diese Abschätzungen bestätigt.

Für die Simulationen wurden dementsprechend Radien gewählt, die ein Verhältnis von $1 / 6$ beibehalten. Als sinnvolle Parameter wurden $r_{s}=35 \AA$ und $r_{k}=210 \AA$ verwendet. Damit sind die Volumina nicht $z u$ groß, so dass ein Simulationsdurchgang im Bereich von Minuten liegt. Gleichzeitig sind sie aber auch groß genug, um Randeffekte weitestgehend zu minimieren.

An dieser Stelle soll der Einfluss, den ein anderes Radienverhältnis auf die Rekonstruktion hat, untersucht werden. Dies geschieht, indem der Abstand von der Al- und der Cr-Ebene in einer $\langle 100\rangle-$ 
orientierten Simulation abhängig von $r_{k}$ und $r_{s}$ bestimmt wird. Dieser Abstand liegt in den Messungen bei $0,83 \AA^{\mathrm{vi}}$. Die anderen Simulationsparameter blieben dabei konstant.

Die Ergebnisse sind in Abbildung 4.11 zu sehen: Wird $r_{k}$ größer, so flacht die Spitze zunehmend ab und das $\Delta z$ aus Formel 3.3 tendiert gegen null. Damit wird der Effekt der verfrühten Evaporation begünstigt, d.h. zwei Atome werden in eine Ebene rekonstruiert, ein Unterschied in der lateralen Position wirkt sich weniger aus und der Abstand der betrachteten Ebenen (also der Peaks) steigt für höhere $r_{k}$.

Nimmt $r_{s}$ andererseits höhere Werte an, wird dieser Effekt ausgeglichen. Im Wesentlichen geschieht dies dadurch, dass aufgrund der größeren Spitzenoberfläche mehr Atome evaporieren können und dadurch die Evaporationsreihenfolge stärker durch die Geometrie und den Feldeinfluss $E_{f}$ bestimmt ist.

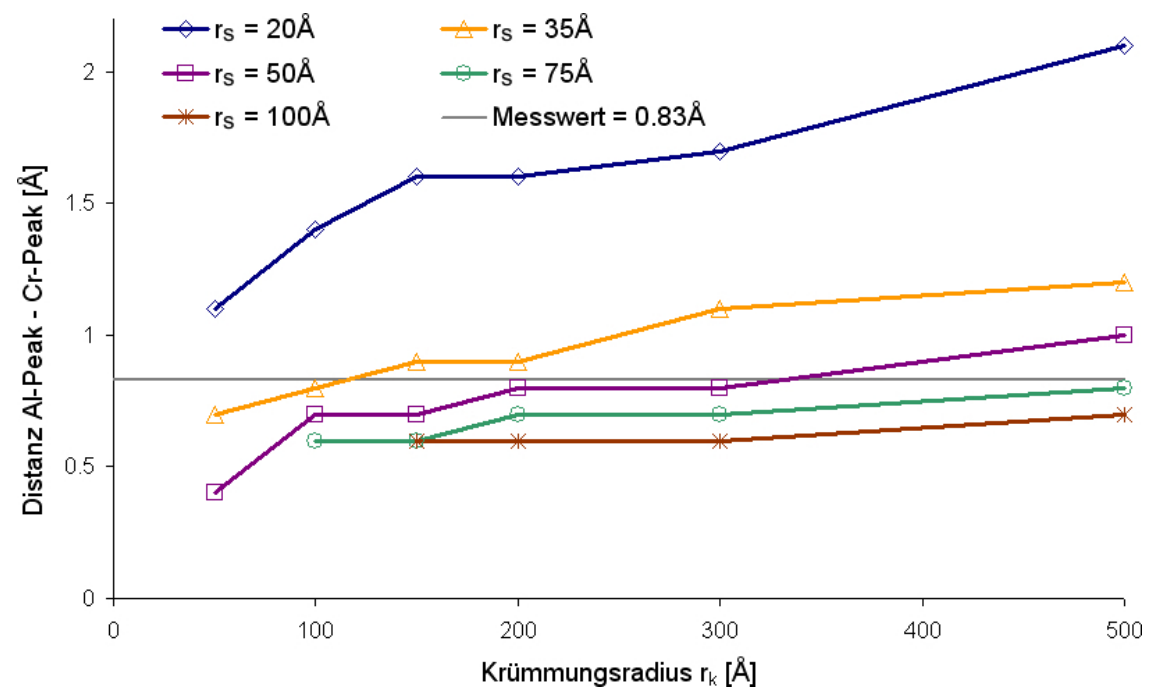

Abbildung 4.11: Einfluss von $r_{k}$ und $r_{s}$ auf die Rekonstruktion des simulierten APT-Volumens

Diese Rechnungen zeigen, dass die Kombination der Radien immensen Einfluss auf das Simulationsergebnis hat. Die obigen Überlegungen stellen also eine wichtige Tatsache für das Rekonstruktionsprinzip und die auf den Simulationen basierenden Analysen dar. Auch die Wahl von $r_{s}=35 \AA$ und $r_{k}=210 \AA$ bildet damit als eine folgerichtige Entscheidung. Weitere mögliche Einflüsse und Ungenauigkeiten aus diesen geometrischen Gegebenheiten werden in 8.2.2 diskutiert.

${ }^{v i}$ Genauer werden die Messungen und Simulationen von $\mathrm{Fe}_{3} \mathrm{Al}-\mathrm{Cr}$ in 5.1 untersucht. 


\section{Kapitel 5: Bestimmung der Platzbesetzung mittels APT-Messungen und -Simulationen}

Die APT-Messungen von den ternären Legierungen werden in diesem Kapitel systematisch untersucht, um die Platzbesetzung der Zusätze zu ermitteln. Ergänzt werden diese Betrachtungen durch die Simulation von APT-Ergebnissen, die zum einen die Analyse zur Platzbesetzung unterstützen und zum anderen weitere Eigenschaften und Rekonstruktionseffekte der Feldevaporation verdeutlichen und Abschätzungen der Ordnungsgrade ermöglichen.

\section{1. $\mathrm{Fe}_{3} \mathrm{Al}-5 \mathrm{at} \% \mathrm{Cr}$}

\subsubsection{Analyse der AtomVicinity-Dichteprofile}

An den chromhaltigen Proben wurden APT-Messungen in alle drei Hauptrichtungen durchgeführt. Analog zu 4.1 wurden daran AtomVicinity-Analysen vollzogen, deren Dichteprofile in z-Richtung in Abbildung 5.1 dargestellt sind. Die Graphen von Eisen und Aluminium sind sehr ähnlich zu denen der binären Legierung, das Verhalten der Peakverschiebung zeigt sich auch hier in einer gleichen Weise.

Um die Platzbesetzung des Chroms nun zu bestimmen, muss man diesen Effekt beachten. Allein in $<110>$-Richtung ist eine nur sehr geringe Peakverschiebung - auch vom Chrom - zu erkennen und die Ebenen klar zu trennen. Allerdings lässt diese Orientierung keine Aussagen über die Chromverteilung zu.

Anders ist der Fall in <111>-Richtung, in der die Ebenenabfolge eine Zuordnung prinzipiell ermöglicht. Hier zeigen das Chrom und das Aluminium eine hohe Übereinstimmung und fast gleiche Peakpositionen, was zunächst einmal die Schlussfolgerung zulässt, dass Al und $\mathrm{Cr}$ gleiche Kristallpositionen einnehmen. Allerdings kann man mit dem Wissen einer möglichen Fehlrekonstruktion (siehe 4.1) nicht ausschließen, dass diese Übereinstimmung nur durch eine Peakverschiebung (z.B. des Chroms) entsteht. Das dies der Fall ist, zeigen zwei Aspekte: Zum einen müssten die durch das Chrom substituierten Aluminiumatome an anderer Stelle sitzen, es ist anzunehmen, dass sie im Wesentlichen auf $\beta$-Plätzen säßen. Dort ist aber kein Peak zu erkennen, wobei davon auszugehen ist, dass dieser zu bemerken wäre, es handelt sich schließlich um $20 \%$ aller Al-Atome (bei vollständiger Substitution). Zum anderen widerspricht auch die Messung in $<100>$-Richtung dieser Schlussfolgerung, hier ist eine Überlagerung von Al- und Cr-Peak nicht gegeben, stattdessen zeigen sie einen deutlichen Abstand.

Folglich scheint die $\gamma$-Platzbesetzung unlogisch. Nähme man eine $\alpha$-Platzbesetzung des Chroms an, würde man in <111>-Richtung zwei Chrom-Peaks zwischen den Al-Peaks erwarten. Den nur einen auftretenden könnte man nur mit einer starken Peakverschiebung nach links und einer dadurch entstehenden Peaküberlagerung bzw. -vereinigung verstehen. In <100>-Richtung hingegen säße der ChromPeak in etwa an der theoretisch erwarteten Stelle, bzw. eher leicht nach rechts verschoben. Dieses unterschiedliche Verhalten einer stark verfrühten Evaporation in <111>-Richtung und einer eher verspäteten Evaporation in $\langle 110>$-Richtung scheint sehr unwahrscheinlich. Auch die $\alpha$ - Platzbesetzung ist daher inkonsistent.

Im Falle einer $\beta$-Platzbesetzung würde man in <111>-Orientierung einen Cr-Peak zwischen den Al-Peaks erwarten. Dass dieser aber an der Position des Aluminium-Peaks ist - bzw. geringfügig rechts davon - 
spräche dann dafür, dass Chrom einer fehlerhaften Rekonstruktion und damit einer Peakverlagerung nach links unterliegt. Dieses Verhalten wäre auch konsistent mit der Messung in <100>-Richtung, hier wäre der Chrom-Peak an der Position des Al-Peaks zu erwarten, er zeigt aber auch hier eine Verschiebung nach links und damit einen gewissen Abstand zum Aluminium-Peak.
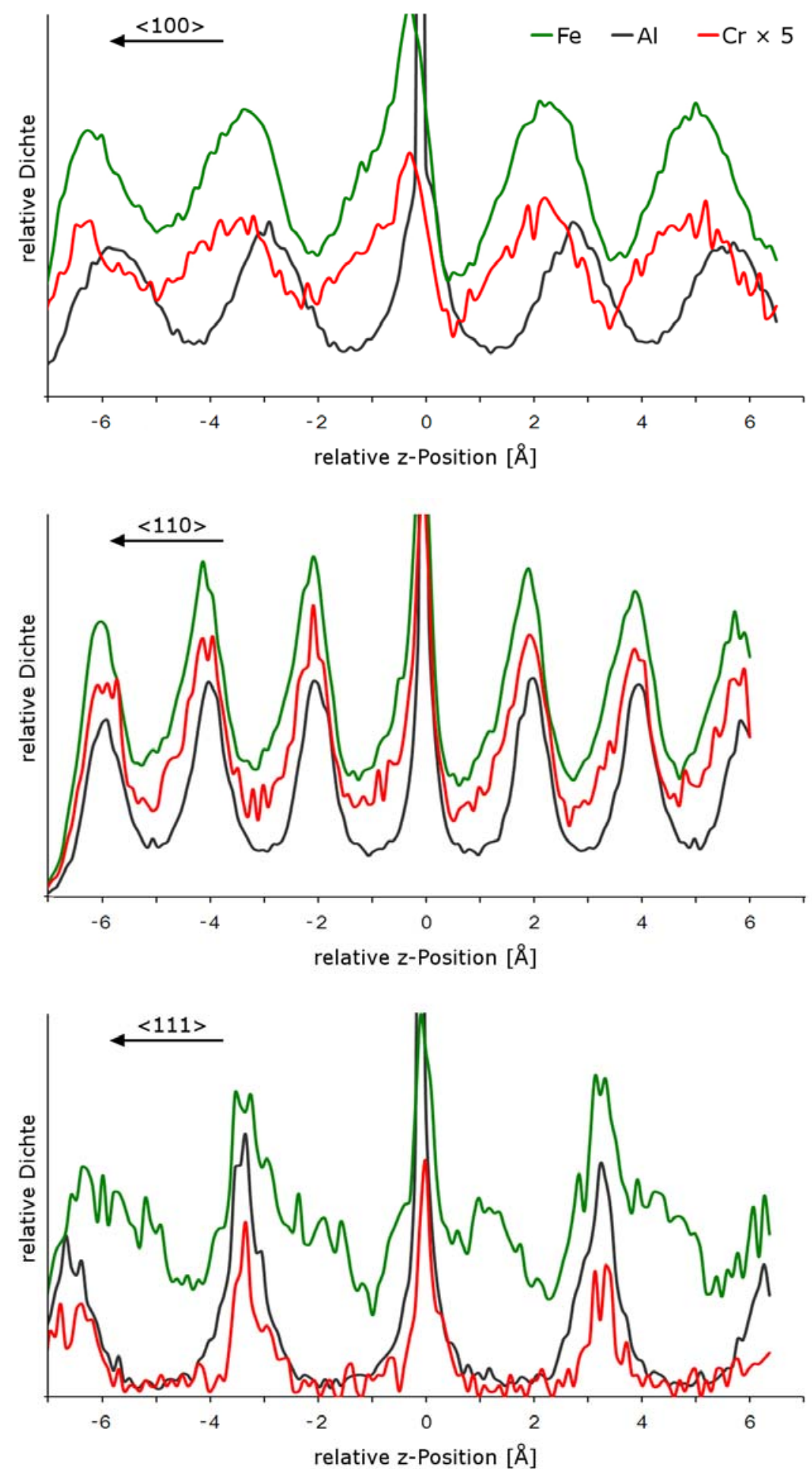

Abbildung 5.1: AtomVicinity-Dichteprofile von APT-Messungen von $\mathrm{Fe}_{3} \mathrm{Al}-\mathrm{Cr}$

Folglich scheint nur die $\beta$-Platzbesetzung eine Ordnung darzustellen, die mit allen Messungen und ihren AtomVicinity-Dichteprofilen in Einklang ist. Dies ist auch in Übereinstimmung zu den meisten bisherigen Studien beschrieben in 2.2. 
Diese Analysen wurden bereits in [65] veröffentlicht und demonstrieren, wie stark sich Rekonstruktionsfehler in diesen Dichteprofilen bemerkbar machen und wie resultierende Peakverlagerungen eine Analyse der Platzbesetzung verkomplizieren können. Um diese Überlegungen zu untermauern und das Verhalten der Chromatome während der Feldverdampfung genauer zu verstehen, sollen die Simulationen der APT-Messungen auch hier angewendet werden.

\subsubsection{Simulationen der Messungen}

Für die Simulationen der APT-Messungen von $\mathrm{Fe}_{3} \mathrm{Al}-\mathrm{Cr}$ müssen neben den in 4.3 verwendeten Parametern nun zusätzlich die Paarpotentiale, an denen Chrom beteiligt ist, bestimmt werden. Auch hier gibt es für das $\mathrm{Fe}-\mathrm{Cr}$ - und das $\mathrm{Cr}$-Cr-Potential diverse Quellen [72, 84, 85], die diese Potentiale zwar geringfügig unterschiedlich, aber jeweils immer sehr ähnlich dem Fe-Fe-Potential beschreiben. Für das Al-Cr-Potential hingegen gibt es in der Literatur nur eine Information [91], in der ein in etwa gemittelter Verlauf von Al-Al-Potential und $\mathrm{Cr}$-Cr-Potential angegeben wird. Im Rah-

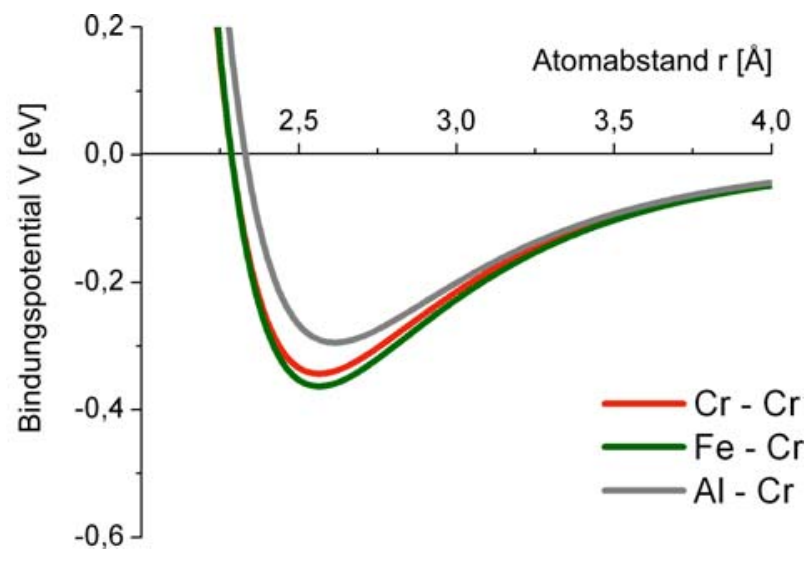

Abbildung 5.2: Paarpotentiale mit Chrom men dieser Angaben wurden die Potentiale auf höchste Übereinstimmung von Simulation und Messung angepasst und sind in Abbildung 5.2 dargestellt.

Die simulierten AtomVicinity-Dichteprofile für eine $\beta$-Platzbesetzung des Chroms zeigt Abbildung 5.3. Die Positionen der Chrom-Peaks entsprechen weitestgehend denen der Messung (Abbildung 5.1). In $<100>$-Richtung kommt es zu einer deutlichen Verschiebung relativ zur nominellen Lage an der Stelle des Aluminium-Peaks. In $<110>-O r i e n t i e r u n g$ sind sehr geringe Verlagerungen der Peaks zu verzeichnen, diese sind damit etwas ausgeprägter als in der Messung. Die Peaks der jeweiligen Elemente sind aber klar einer gemeinsamen Ebene zuzuordnen. In <111>-Richtung hingegen ist die erwartete Verschiebung des Chrom-Peaks erkennbar, er ist deutlich weiter links positioniert und überlagert den Aluminium-Peak nahezu.

In diesen Simulationen wurden die Ordnungsgrade der Al- und Cr-Atome bereits auf eine höchstmögliche Übereinstimmung optimiert, sie liegen demnach bei $\xi_{A l, \gamma}=0,94 \pm 0,02$ für Aluminium bzw. $\xi_{c r, \beta}=0,83 \pm 0,05$ für Chrom. Eine genauere Betrachtung und Diskussion dazu folgt in 8.3.2. 

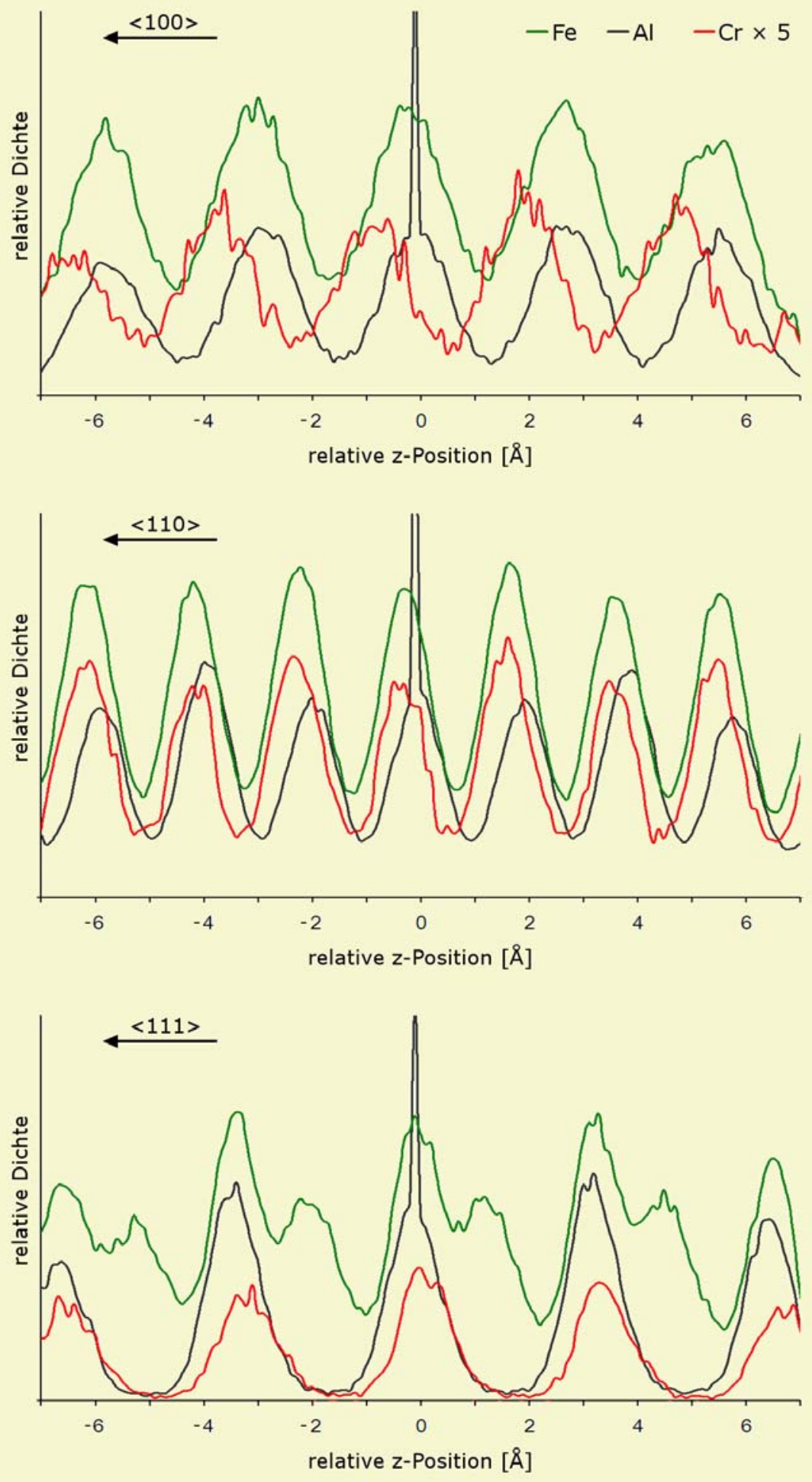

Abbildung 5.3: AtomVicinity-Dichteprofile simulierter APT-Messungen von $\mathrm{Fe}_{3} \mathrm{Al}-\mathrm{Cr}$ mit $\beta$-Platzbesetzung

Damit kann gezeigt werden, dass die Überlegungen zur Peakverschiebung aus 5.1.1 und der damit verbundenen $\beta$-Platzbesetzung ein realistisches Verhalten darstellen. Um die Analyse dennoch zu festigen, ist eine Gegenprobe sinnvoll, d.h. eine Simulation von APT-Messungen mit einer anderen Platzbesetzung. Als ein Gegenbeispiel dient Abbildung 5.4a, in der eine $\gamma$-Platzbesetzung des Chroms simuliert wurde. Die Aluminiumatome, die dabei durch Chrom ersetzt wurden, wurden gleichmäßig auf $\alpha$ - und $\beta$-Plätzen verteilt. Die bereits beobachtete Peakverlagerung des Chroms relativ zu den AluminiumPeaks findet auch hier statt, so dass Al- und Cr-Peaks einen deutlichen Abstand aufweisen. Eine Über- 

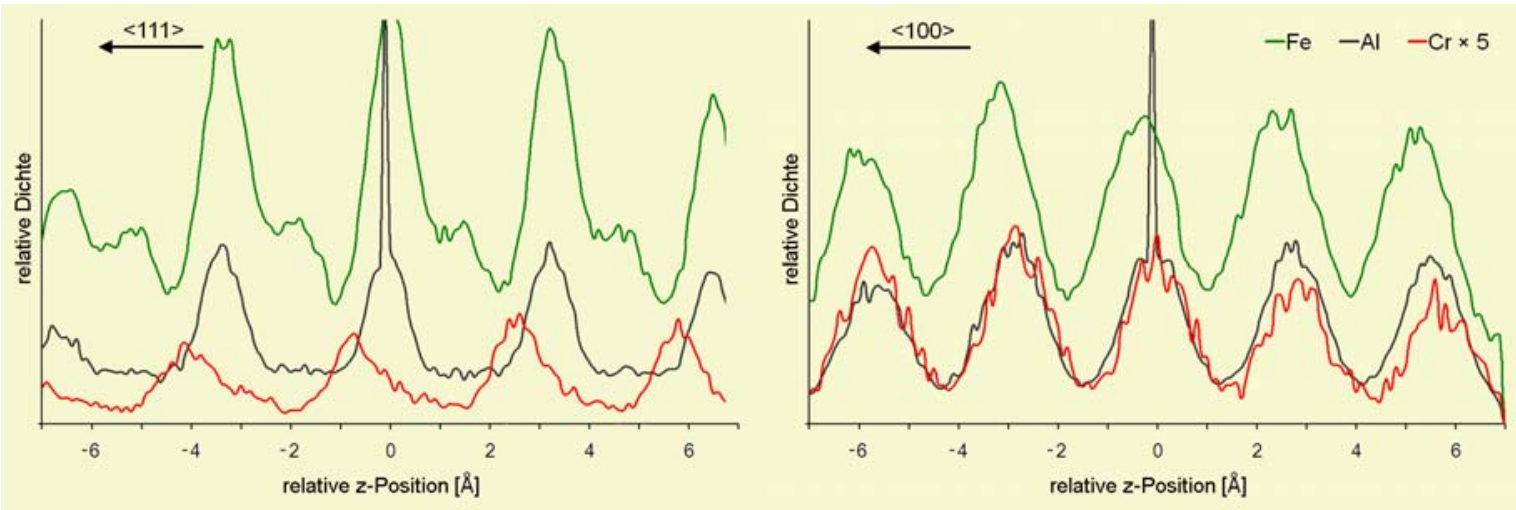

Abbildung 5.4: AtomVicinity-Dichteprofil simulierter APT-Messungen mit Chrom auf a) $\gamma$-Plätzen (links) und b) $\alpha$-Plätzen (rechts)

einstimmung mit den Messungen aus Abbildung 5.1 ist kaum mehr gegeben. Außerdem ist durch das substituierte Aluminium dessen Peak-Rauschsignal-Verhältnis deutlich anders als in den Messungen.

Ein anderes Gegenbeispiel mit einer $\alpha$-Platzbesetzung zeigt Abbildung 5.4b. Hier ist eine $<100>-$ Orientierung dargestellt. Auch hier findet wiederum eine Peakverschiebung statt, wodurch sich Al- und $\mathrm{Cr}$-Peak an gleichen Positionen befinden. Eine solche Überlagerung entspricht aber gleichfalls nicht den Messungen.

Man könnte diese Abweichungen aus Abbildung 5.4 zu den Messungen nur noch dadurch erklären, dass die Bindungsparameter - also insbesondere die Paarpotentiale - als zu schwach angenommen wurden. Wären diese stärker, könnte in der Tat jeweils das gemessene Profil simuliert werden. Dann würden aber jeweils andere Richtungen $(<111>$-Richtung bei $\alpha$-Platzbesetzung; $<100>$-Richtung bei $\gamma$ Platzbesetzung) keine Peakverschiebung zeigen und damit nicht den entsprechenden Messungen gleichen.

Letztendlich zeigen die Simulationen, dass nur eine $\beta$-Platzbesetzung konsistent ist. Außerdem bestätigen sie die Überlegungen zu Peakverschiebung aus 5.1.1 und damit den Effekt der verfrühten Evaporation der Chromatome.

\section{2. $\mathrm{Fe}_{3} \mathrm{Al}-5 \mathrm{at} \% \mathrm{Mn}$}

\subsubsection{Analyse der AtomVicinity-Dichteprofile}

Die Profile der manganhaltigen Legierung (Abbildung 5.5) sind denjenigen vom $\mathrm{Fe}_{3} \mathrm{Al}-\mathrm{Cr}$ sehr ähnlich. Wiederum sind die Positionen der Peaks bei Aluminium und Eisen vergleichbar mit denen der binären Legierung. Die Manganatome sind in $<110\rangle$ - und $<111>$-Richtung ungefähr beim Aluminium positioniert, in $<100>$-Orientierung zeigen sie zu den Aluminium-Peaks eine gewisse Distanz.

Die Analyse dieser drei Profile kann ganz analog zu der aus 5.1.1 der chromhaltigen Proben geführt werden:

- Gegen eine $\gamma$-Platzbesetzung (Al-Plätze) spricht, dass Mangan und Aluminiumatome in <111>Richtung gleich positioniert sind, nicht aber in $\langle 100\rangle$-Richtung. Außerdem sind die Al-Atome, die durch das Mangan substituiert werden würden, kaum auf anderen Plätzen auszumachen.

- Eine $\alpha$-Platzbesetzung würde bedeuten, dass in $<111>-$ Richtung die zwei Mangan-Peaks eine starke Verschiebung nach links auf eine gemeinsame Position erfahren müssten. In der <100>Orientierung würde sie hingegen eine leichte Verschiebung nach rechts darstellen. Diese stark unterschiedlichen Evaporationsverhalten sind höchst unwahrscheinlich. 
- Eine $\beta$-Platzbesetzung würde sowohl für die $<111>$ - als auch für die $<100>-$-Orientierung eine jeweilige Verschiebung nach links bedeuten, was ein konsistenteres Verhalten darstellt und für eine relativ geringe Feldverdampfungsfeldstärke des Mangans spricht. In <110>-Richtung ist diese Peakverschiebung abermals nur sehr gering ausgeprägt, eine kleine Verschiebung nach links (relativ zu Aluminium) ist aber zu erkennen.

Folglich ist für das Mangan ebenfalls eine Besetzung der $\beta$-Plätze die einzige Ordnung, die mit allen drei Profilen eine hohe Vereinbarkeit liefert.
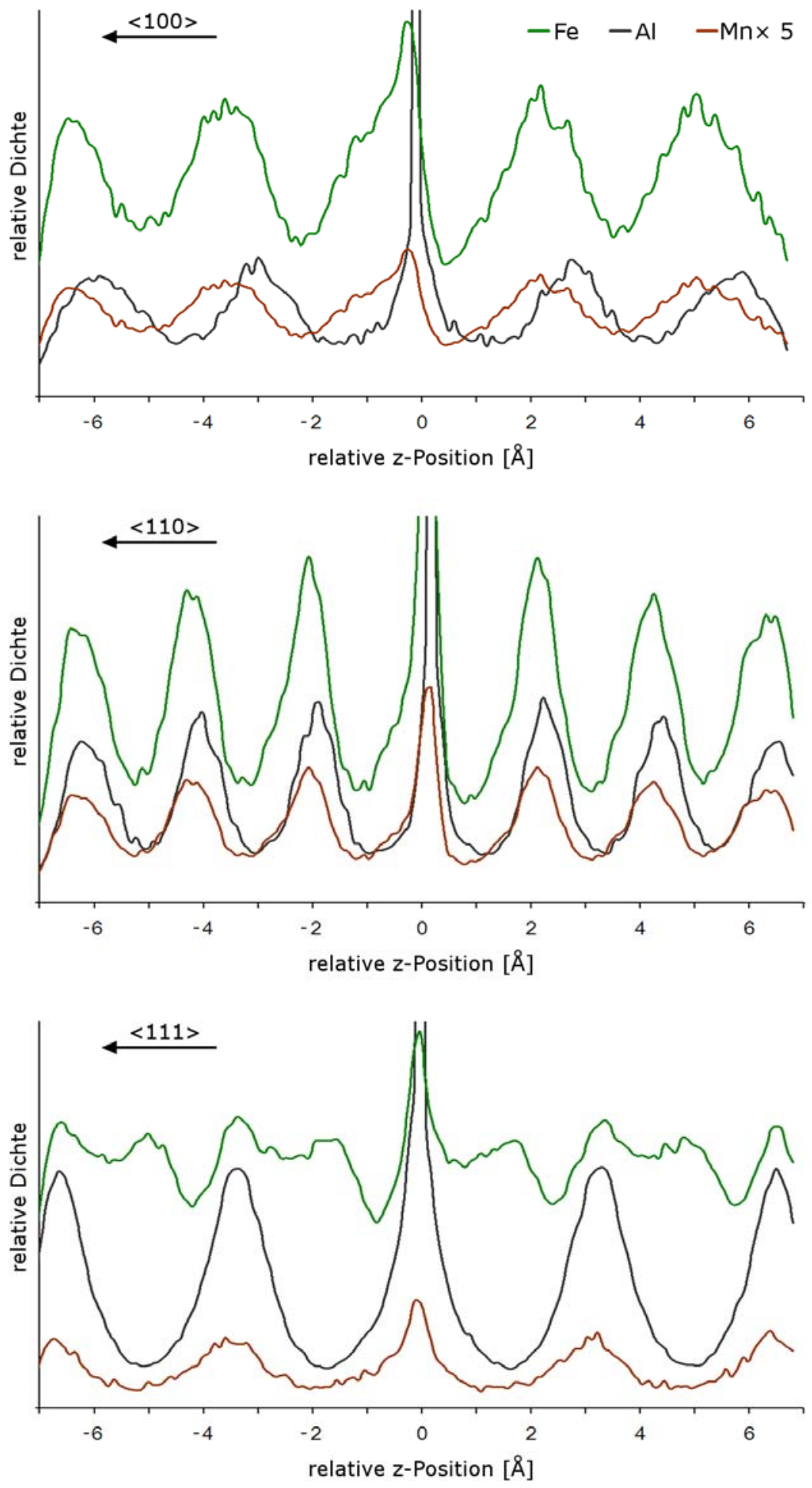

Abbildung 5.5: AtomVicinity-Dichteprofile von APT-Messungen von $\mathrm{Fe}_{3} \mathrm{Al}-\mathrm{Mn}$ 


\subsubsection{Simulationen der Messungen}

Für die Simulationen müssen nun wiederum die Paarpotentiale, an denen Mangan beteiligt ist, ermittelt werden. In der Literatur sind keine entsprechenden Potentiale nach dem EAM-Formalismus zu finden. Allerdings gibt es andere Berechnungen mit geringfügig anderen Modellen ${ }^{\text {vii }}$ [93, 89]. Deren Paarpotentiale können zwar nicht mit Absolutwerten verwendet werden, ihre Stärken können aber für relative Abschätzungen dienlich sein. So ist das $\mathrm{Mn}-\mathrm{Mn}$-Potential im Vergleich etwas geringer, das FeMn-Potential etwa gleich stark wie das Fe-Fe-Potential. Das Al-Mn-Potential hat demnach ein tieferes Minimum als die jeweiligen Potentiale der beteiligten reinen Elemente [89]. Insbesondere die Lage der Minima kann aus den genannten Quellen entnommen werden. Mit diesen Vorgaben erfolgte eine bestmögliche Anpassung der Simulationen an die Messungen. Die resultierenden Paarpotentiale sind nebenstehend dargestellt, die entsprechenden AtomVicinity-Dichteprofile der simulierten Messungen zeigt Abbildung 5.7.

Deutlich zeigt sich auch hier die Verschiebung des Mangan-Peaks nach links in $<100>$ - und $<111>$-Richtung, die Peakpositionen stimmen damit gut mit den Messungen überein. Die

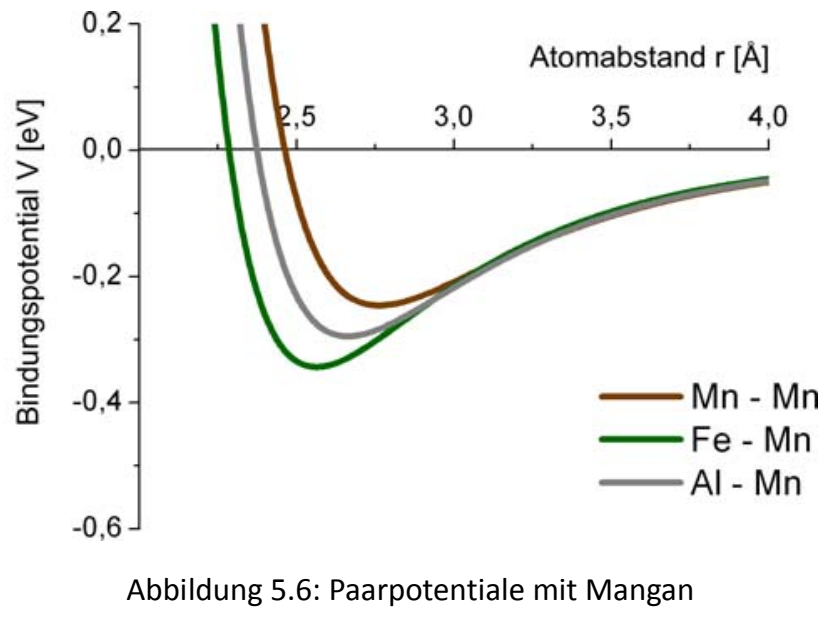
Form der Peaks hingegen zeigt teilweise gewisse Unterschiede. In <110>-Orientierung ist ebenfalls eine leichte Linksverlagerung des ManganPeaks zu erkennen, wenn auch deutlich weniger stark ausgeprägt als bei den anderen beiden Richtungen. Ein solches Verhalten ist zwar auch bei den Messungen zu erkennen, jedoch nicht so auffällig wie in den Simulationen. Dennoch können auch hier alle Peaks klar einer Ebene zugeordnet werden.

Damit können die Überlegungen zum Evaporationsverhalten und zur Platzbesetzung aus 5.2.1 durch die Simulationen bestätigt werden und insgesamt auch für $\mathrm{Fe}_{3} \mathrm{Al}-\mathrm{Mn}$ eine $\beta$-Platzbesetzung festgehalten werden.

Die Ordnungsgrade können für diese Legierung bei Aluminium zu $\xi_{\mathrm{Al}, \mathrm{\gamma}}=0,94 \pm 0,05$ und bei Mangan zu $\xi_{\mathrm{Mn}, \beta}=0,75 \pm 0,10$ bestimmt werden.

vii Diese können Abweichungen des EAM-Formalismus sein, die zur genaueren Berechnung bestimmter Eigenschaften weitere Terme enthalten. Dazu zählt z.B. auch die MEAM (Modified Embedded Atom Method-Modifizierte EAM) [92]. 

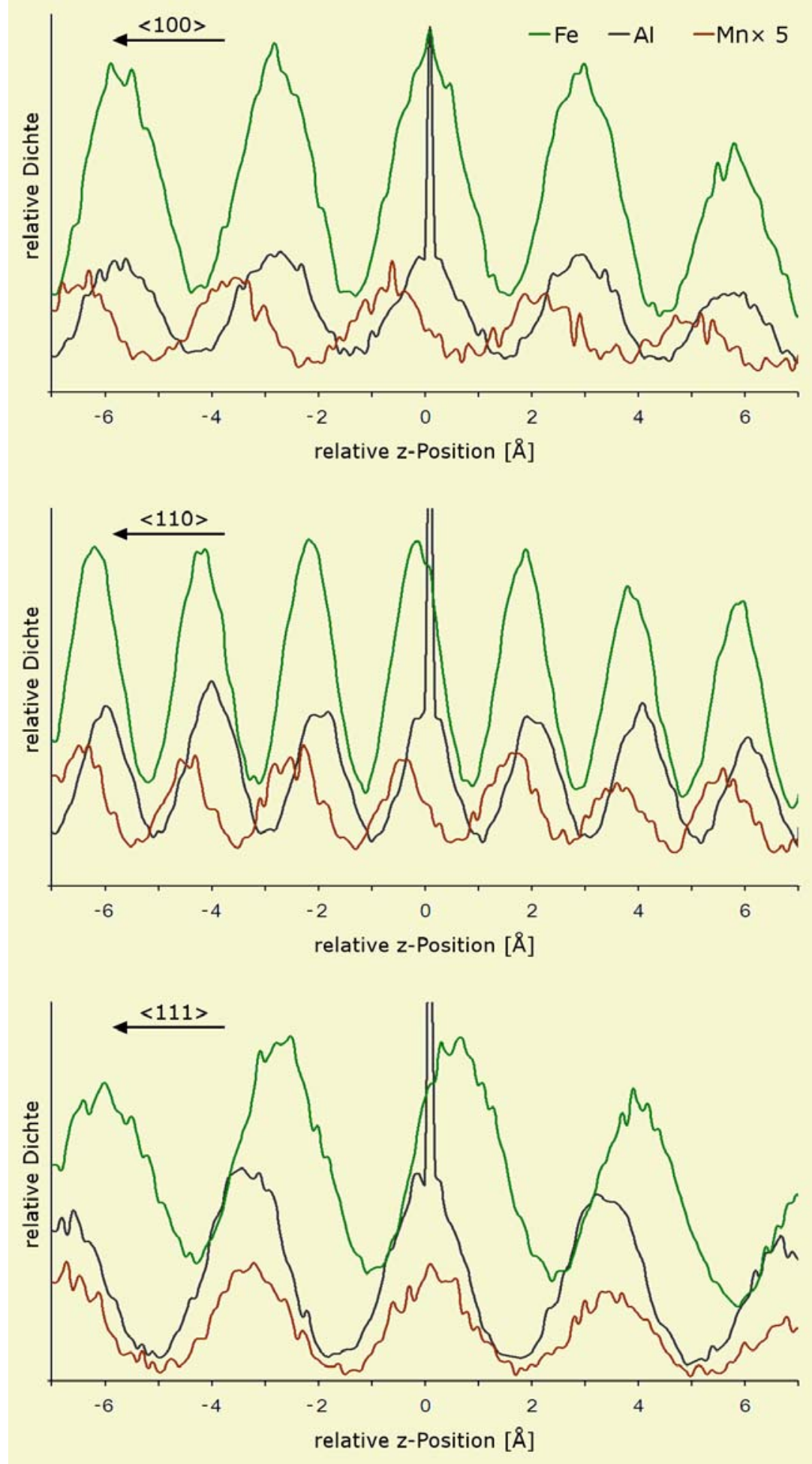

Abbildung 5.7: AtomVicinity-Dichteprofile simulierter APT-Messungen von $\mathrm{Fe}_{3} \mathrm{Al}-\mathrm{Mn}$ mit $\beta$-Platzbesetzung 


\section{3. $\mathrm{Fe}_{3} \mathrm{Al}-5 \mathrm{at} \% \mathrm{~V}$}

\subsubsection{Analyse der AtomVicinity-Dichteprofile}

Abbildung 5.8 zeigt die AtomVicinity-Dichteprofile der APT-Messungen an $\mathrm{Fe}_{3} \mathrm{Al}-\mathrm{V}$. Sie unterscheiden sich teilweise signifikant von den bisher betrachteten Dichteprofilen. So sind in $<100>-$ Richtung die Peaks von Vanadium und Aluminium nahezu an gleichen Orten positioniert. In <110>-Orientierung liegen die Peaks aller Elemente in etwa an gleichen Stellen, so wie es auch bei den chrom- und man-
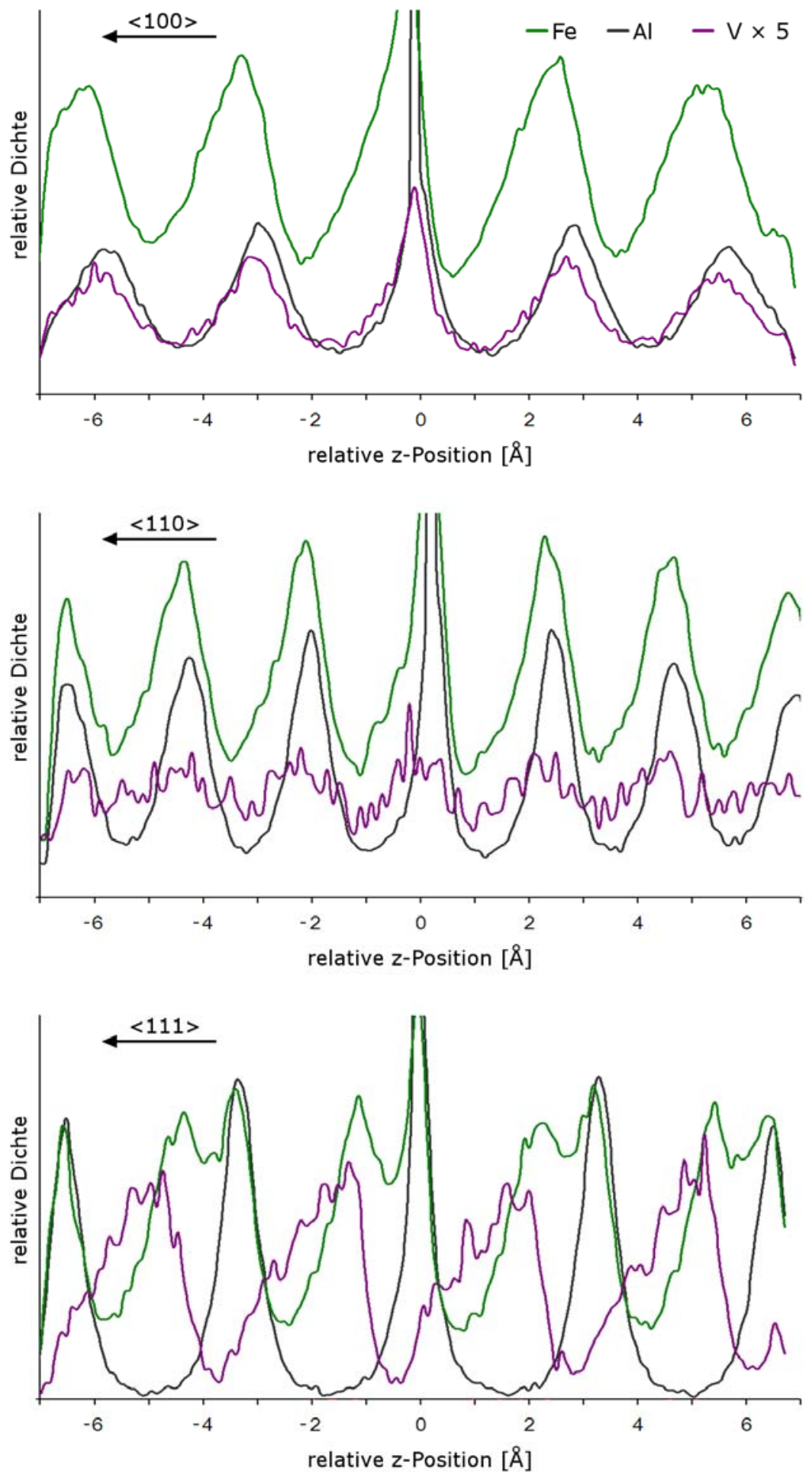

Abbildung 5.8: AtomVicinity-Dichteprofile von APT-Messungen von $\mathrm{Fe}_{3} \mathrm{Al}-\mathrm{V}$ 
ganhaltigen Proben zu erkennen war. Hier sind die Vanadium-Peaks relativ unscharf, wenngleich ihre Positionen eindeutig erkannt werden können.

Ein deutlich anderes Bild zu allen anderen Messungen in <111>-Richtung zeigt diejenige mit Vanadium. Hier ist der Verlauf des Eisens vollkommen unterschiedlich. Die drei Eisen-Peaks, die (nach den theoretischen Dichteprofilen aus 4.1) zwischen den Aluminium-Peaks liegen, sind wenigstens teilweise nach rechts verschoben anstatt nach links. Dabei gibt es zwei Möglichkeiten: Entweder sind alle drei EisenPeaks nach rechts verlagert und bilden eine gemeinsame Erhebung. Die andere Möglichkeit besteht darin, dass nur der rechte (und evtl. der mittlere) Eisenpeak nach rechts verschoben ist, der linke jedoch immer noch nach links verschoben ist, so dass sich dazwischen eine Lücke bildet und daraus die ermittelte Peakform resultiert. Es ist nicht eindeutig zu erkennen, welcher der Fälle eintritt, letzterer erscheint in Anbetracht der bisherigen Erkenntnisse als wahrscheinlicher. Insgesamt kann aber festgestellt werden, dass sich das Eisen, welches in den anderen Messungen nur nach links verschoben (also verfrüht evaporierend) bestimmt wurde, hier anders verhält. Wenigstens ein Teil des Eisens unterliegt in dieser Legierung einer Verschiebung nach rechts und damit einer verspäteten Evaporation. Es ist aber bemerkenswert, dass dieser Effekt nur in der $<111>$-Orientierung auftritt, in der $<100>$-Richtung ist eine Linksverschiebung wie bei allen anderen Messungen der $<100>$-Richtung zu erkennen.

Um die Platzbesetzung des Vanadiums zu bestimmen, sollen auch hier wieder alle drei möglichen Fälle systematisch untersucht werden. Der Fall einer $y$-Platzbesetzung (Al-Plätze) ist dabei am eindeutigsten: Auch wenn dies mit den Messungen der $<100>$ - und $<110>$-Richtung Übereinstimmungen in den Peakpositionen ergäbe, so spricht die <111>-orientierte Messung dagegen. Zum einen würde dies bedeuten, dass Vanadium einer stark verfrühten oder verspäteten Evaporation unterliegt. Diese wäre aber in der $<100>$-Richtung eben nicht zu erkennen. Zum anderen müssten auch hier wieder die durch Vanadium substituierten Al-Atome an anderer Stelle zu finden sein. Zwischen den Aluminium-Peaks fällt dessen Konzentration aber auf nahezu null ab. Damit erscheint eine $\gamma$-Platzbesetzung nicht konsistent mit den Messungen.

Betrachtet man eine $\alpha$-Platzbesetzung, würde man für die $<111>-$ Richtung theoretisch zwei V-Peaks zwischen den Aluminium-Peaks erwarten. Zu erkennen ist nur ein breiter V-Peak. Man könnte allerdings annehmen, dass die zwei Vanadium-Peaks eine Überlagerung zeigen und sich so zu einem Peak vereinigen. Dieser würde dann eine geringe Linksverschiebung zeigen. Eine solche Platzbesetzung bedeutete aber, dass in <100>-Orientierung das Vanadium zwischen den Al-Peaks zu finden sein müsste, was nicht der Fall ist. Daher müsste man für diese Orientierung von einer stark verfrühten oder stark verspäteten Evaporation ausgehen. Letztere wäre dabei eher anzunehmen, da sich der Vanadium-Peak geringfügig links vom Aluminium-Peak befindet. Insgesamt würde eine $\alpha$-Platzbesetzung aber ein deutlich unterschiedliches Evaporationsverhalten für die $<111>-$ und die $<100>$-Richtung bedeuten, was als eher unwahrscheinlich eingestuft werden kann.

Die Ordnung mit der höchsten Konsistenz zu den Messungen ist wiederum die $\beta$-Platzbesetzung. In diesem Fall befinden sich in allen Richtungen die Vanadium-Peaks an den erwarteten Positionen. Allein in <111>-Richtung gibt es eine leichte Verschiebung nach links. Jedoch erscheint diese insbesondere durch die unsymmetrische Form des Peaks, sein Schwerpunkt befindet sich relativ genau mittig zwischen den Peaks des Aluminiums. Diese $\beta$-Platzbesetzung würde dann bedeuten, dass Vanadium ein ähnliches Evaporationsverhalten wie das Aluminium aufweist und damit relativ zu diesem keine Peakverlagerung erfährt. 


\subsubsection{Simulationen der Messungen}

Um nun Simulationen der vanadiumhaltigen Proben zu erhalten, müssen wiederum die benötigten Paarpotentiale bestimmt werden. Für das V-V-Potential sind mehrere Quellen zu finden $[72,84,86]$, die das Potentialminimum bei ca. 2,7Å mit einer Tiefe von 0,31eV - 0,45eV angeben. MENDELEV beschrieb ebenfalls das Fe-V-Potential als ein sehr starkes Potential mit einem Energieminimum von 0,55eV im Abstand von ca. 2,5Å. Für das Al-V-Potential konnten keine Literaturwerte gefunden werden.

Alle Potentiale wurden im Bereich dieser Vorgaben wiederum auf eine höchstmögliche Übereinstimmung zwischen Messung und Simulation angepasst, sie sind nebenstehend in Abbildung 5.9 dargestellt.

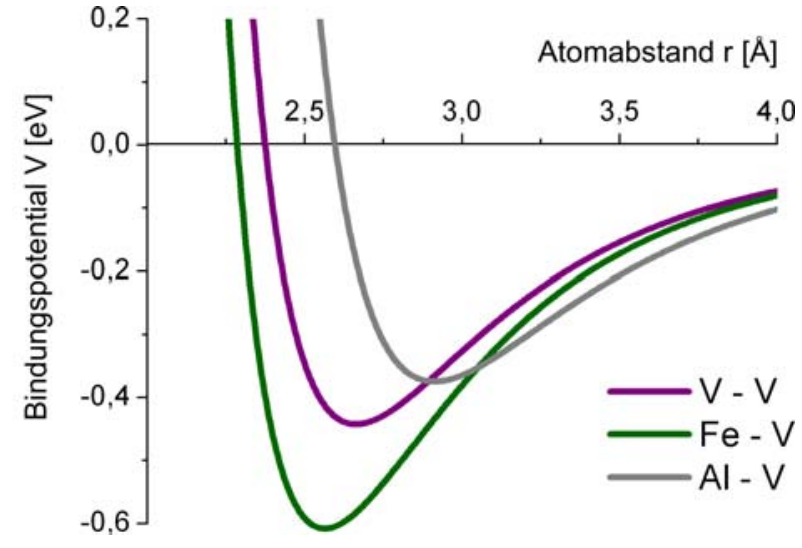

Abbildung 5.9: Paarpotentiale mit Vanadium

Die AtomVicinity-Dichteprofile der simulierten APT-Messungen von Vanadium auf $\beta$-Plätzen zeigt Abbildung 5.10. Insbesondere für die $<100>$-Richtung zeigt sich eine hohe Übereinstimmung zwischen der Messung und der Simulation. Die Peaks von Vanadium, Aluminium und Eisen sind jeweils gleich positioniert. In <110>-Orientierung zeigen sich auch wieder nur sehr geringe Peakverschiebungen, die Peaks einer Ebene sind dicht beieinander. Ein geringfügiger Unterschied zu den Messungen besteht darin, dass der Vanadium-Peak etwas rechts neben dem des Aluminiums sitzt und nicht links, die abso-

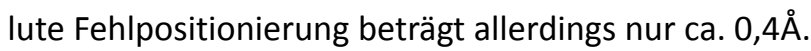

Interessant ist das Dichteprofil für die <111>-Richtung. Hier sind die Vanadium-Peaks zwischen den Aluminium-Peaks positioniert und damit an den erwarteten Stellen und in Übereinstimmung zu den Messungen. Unterschiedlich sind jedoch die Peakformen. Der gemessene, relativ scharf abgegrenzte VPeak mit einer Flanke an der linken Seite konnte so nicht simuliert werden. Da das Entstehen der gemessenen Peakform nicht eindeutig zu erklären ist, muss diese Tatsache vorerst so hingenommen werden. Eine detailliertere Diskussion zu diesem Phänomen wird in 8.2 vorgenommen.

Das Eisenprofil der <111>-Orientierung weist - genau wie bei den Messungen - eine starke Veränderung zu den anderen simulierten Profilen für diese Richtung auf. Der rechte der drei Eisen-Peaks ist im Diagramm nach rechts verlagert, erfährt also eine verspätete Feldverdampfung. Dadurch bildet das Eisen das resultierende Gesamtprofil. Die Simulation kann damit das gemessene Profil des Eisens prinzipiell nachahmen. Wenngleich es gewisse Unterschiede in der genauen Peakform gibt, kann der Effekt, dass in dieser Orientierung ein Teil des Eisens verspätet evaporiert, durch die Simulation nachempfunden werden.

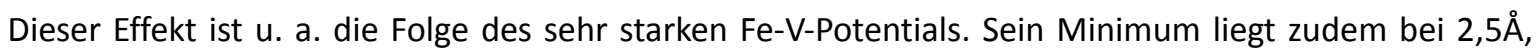
was sehr genau dem NN-Abstand der hier betrachteten Struktur entspricht $\left(d_{N N}=2,49 \AA\right)$. Dies führt zu einer stärkeren Bindung für bestimmte Eisenatome und beeinflusst deren Evaporationsverhalten stark. Diese Tatsache ist dahingehend bemerkenswert, dass dieser Effekt durch die Beimischung von nur 5 at\% Vanadium hervorgerufen wird. 

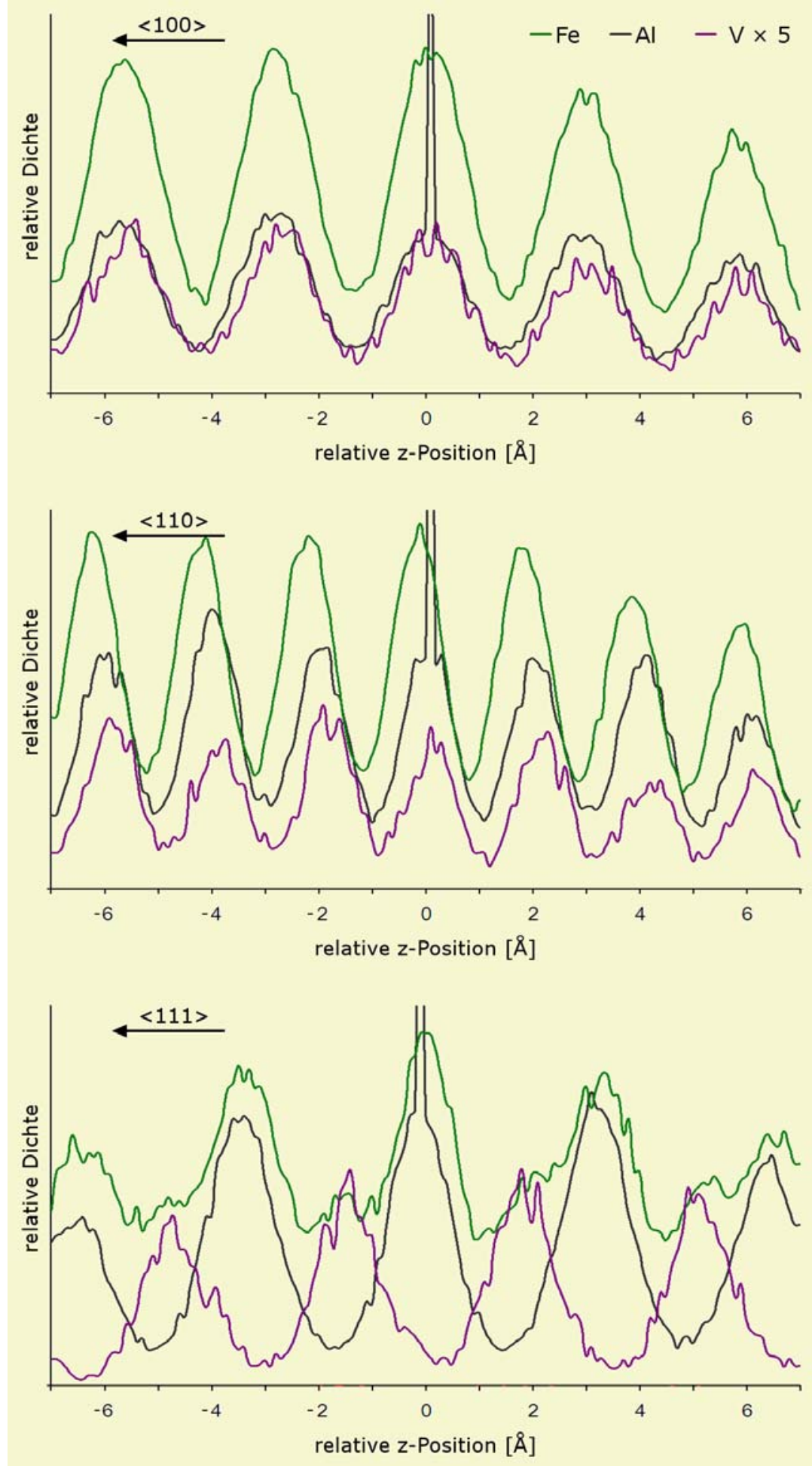

Abbildung 5.10: AtomVicinity-Dichteprofile einer simulierten APT-Messung von Fe ${ }_{3} \mathrm{Al}-\mathrm{V}$ mit $\beta$-Platzbesetzung

Die Ordnungsgrade können für diese Legierung im Vergleich zu den vorhergehenden Untersuchungen nur weniger präzise festgesetzt werden. Dies liegt insbesondere an der nicht so deutlich zu erfassenden $<111>$-Richtung, bei der die Simulationen gewisse Abweichungen zur Messung aufzeigen. Dadurch kann die Vanadiumordnung nur ungenauer $z u \xi_{v, \beta}=0,85 \pm 0,15$ bestimmt werden. Die $<100>$-Richtung ist zwar besser zu simulieren, aber auch weniger aussagekräftig, da hier eine Fehlpositionierung von Vanadium auf $\gamma$-Plätzen kaum in Erscheinung tritt. Der Ordnungsgrad von Aluminium kann auf $\xi_{\mathrm{Al}, \mathrm{Y}}=0,93 \pm 0,07$ festgesetzt werden. 


\section{4. $\mathrm{Fe}_{3} \mathrm{Al}-5 \mathrm{at} \% \mathrm{Ti}$}

\subsubsection{Analyse der AtomVicinity-Dichteprofile}

APT-Messungen und auch rudimentäre Simulationen für die Legierung $\mathrm{Fe}_{3} \mathrm{Al}-\mathrm{Ti}$ wurden im Rahmen eines gemeinsamen Projektes der Deutschen Forschungsgemeinschaft (DFG, Projektnummer AL 592/3-1) bereits von KRESSE [94] durchgeführt. Diese werden in dieser Arbeit durch weitere Messungen und Simulationen mithilfe des weiterentwickelten Simulationsalgorithmus ergänzt.
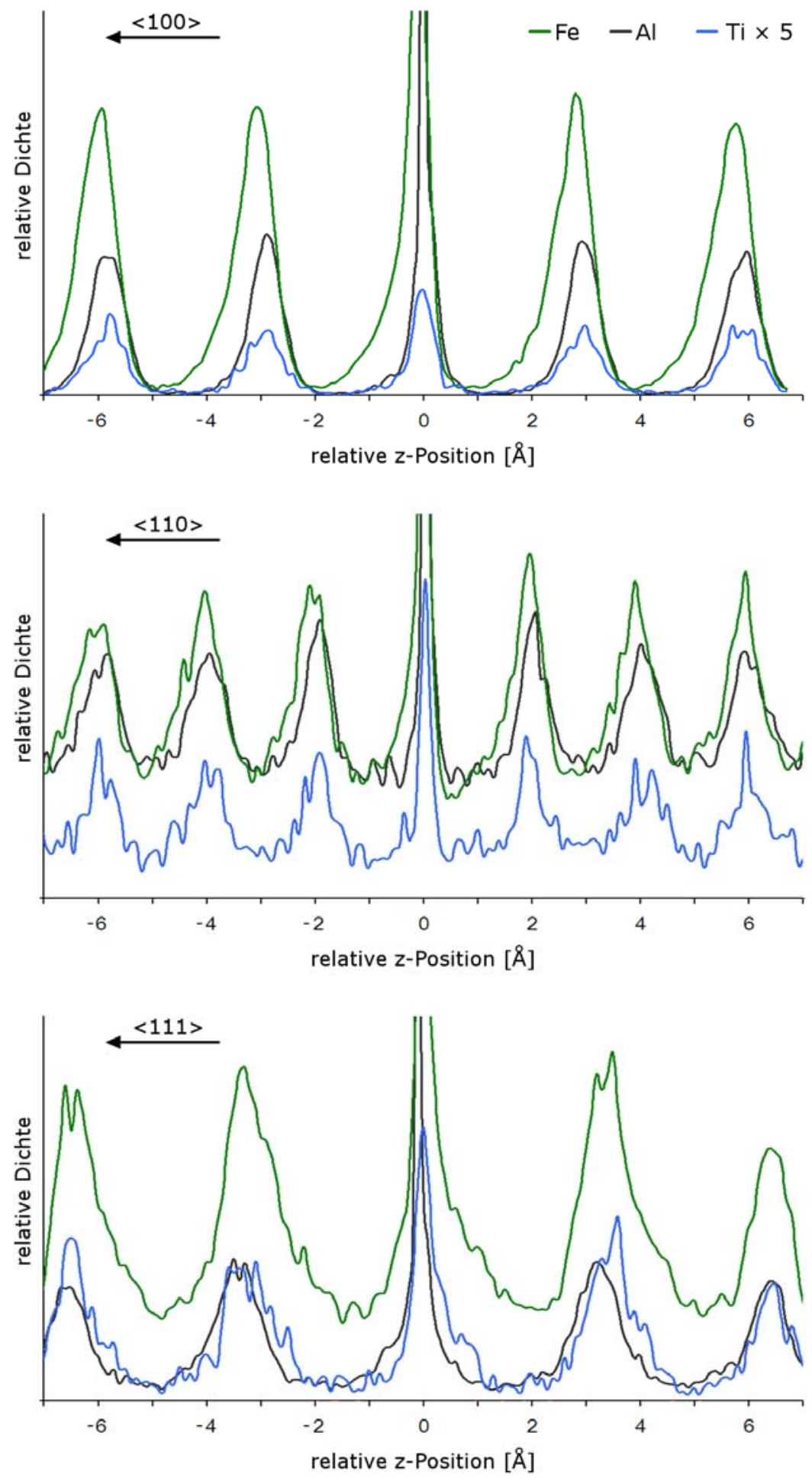

Abbildung 5.11: AtomVicinity-Dichteprofile von APT-Messungen von $\mathrm{Fe}_{3} \mathrm{Al}-\mathrm{Ti}$.

Die Messungen der oberen beiden Grafiken wurden durchgeführt von KRESSE [94]. 
KRESSE schlussfolgerte aus AtomVicinity-Dichteprofilen von $\mathrm{Fe}_{3} \mathrm{Al}-\mathrm{Ti}$ für die $<100>-$ und $<110>-$ Orientierug und deren Simulationen, in Kombination mit Leiterdiagrammen dieser Messungen, dass Titan die $\beta$-Plätze besetzen müsse.

Im Rahmen dieser Arbeit wurde u. a. zusätzlich die <111>-Richtung mit der APT gemessen. Alle AtomVicinity-Dichteprofile der Messungen der drei Hauptrichtungen sind in Abbildung 5.11 dargestellt. Allgemein betrachtet kann man feststellen, dass die Peakpositionen und -formen denen der binären Legierung entsprechen, d.h. das Eisen zeigt relativ zum Aluminium eine Peakverschiebung nach links in der $<100>$ - und $<111>$-Richtung. Weiterhin erscheinen für diese beiden Richtungen die Peaks schärfer, diese Tatsache ist bei allen Elementen festzustellen. Dies kann durch die höhere Messtemperatur erklärt werden. Da gerade die titanhaltige Legierung sehr spröde ist, konnten erfolgreiche Messungen nur bei 70K durchgeführt werden, um ein Abbrechen der Probenspitze zu vermeiden. Dies kann Auswirkungen auf das Evaporationsverhalten und damit verbunden auch auf die Peakform und -schärfe haben. Genauer werden diese Einflüsse in 8.2.2 diskutiert.

Die Platzbesetzung des Titans soll nun anhand dieser Messungen analysiert werden. Auffällig ist, dass in allen drei Richtungen die Peaks von Titan und Aluminium an nahezu gleichen Positionen sitzen. Damit wäre es naheliegend, dass Titan Al-Plätze besetzt, also $\gamma$-Plätze. Dagegen spricht jedoch abermals, dass die substituierten Aluminiumatome nicht an anderer Stelle detektiert werden. In diesem Fall müssten sich $16 \%$ der Al-Atome auf anderen Kristallplätzen befinden, es sind jedoch zwischen den AlPeaks keine kleinen Peaks auszumachen, bzw. hat es aufgrund der Form dieser Peaks nicht den Anschein, dass sich dazwischen eine signifikante Anzahl an Al-Atomen befindet. Außerdem ist die Form der Peaks von Aluminium und Titan sehr komparabel. Dies wäre aber nicht zu erwarten, wenn bei Titan eine reine $\gamma$-Platzbesetzung und bei Aluminium eine $\gamma$ - und $\alpha$-/ $\beta$-Platzbesetzung vorkäme. Denn wenn alle Atome des Titans auf $\gamma$-Plätzen sitzen, vom Aluminium jedoch maximal 84\% (und der Rest auf anderen Plätzen), ist es unlogisch, dass die Peakformen beider Elemente so ähnlich sind. Stattdessen müsste man einen unschärferen Al-Peak erwarten und/oder ein höheres, relatives Signal zwischen den Peaks. Mit diesen Überlegungen kann eine $y$-Platzbesetzung des Titans als sehr unwahrscheinlich eingestuft werden.

Betrachtet man nun eine $\alpha$-Platzbesetzung, würde das für die $<111>-$ Orientierung bedeuten, dass man zunächst zwei Ti-Peaks zwischen den Aluminium-Peaks erwarten würde. Da man nur einen an der Stelle des Aluminiums erkennt, müsste man von einer stark verfühten Evaporation ausgehen, die dafür sorgt, dass die Ti-Peaks deutlich nach links verschoben werden und sich zu einem Peak überlagern. Ein solches Verhalten wäre mit der $<100>$-Richtung konsistent, hier würde man einen Peak zwischen den Al-Peaks erwarten. Dass er aber ebenfalls an der Stelle des Al-Peaks positioniert ist, lässt wiederum auf eine starke Peakverschiebung schließen. Damit erscheint die $\alpha$-Platzbesetzung durch Titan möglich.

Zuletzt soll nun eine $\beta$-Platzbesetzung betrachtet werden. Für die $\langle 100>$-Richtung würde dies bedeuten, dass die Titan-Peaks genau an den erwarteten Stellen sitzen, überlagernd mit dem Aluminium. In $<111>$-Richtung hingegen wären die zwischen den Al-Peaks erwarteten Titan-Peaks deutlich nach links verlagert. Nun ist zu erkennen, dass in dieser Orientierung die Titan-Peaks in der Tat geringfügig rechts von den Al-Peaks sitzen. Dass würde bedeuten, dass hier die Peakverlagerung nach links nicht so stark ausgeprägt wäre wie beispielsweise bei der manganhaltigen Probe (siehe Abbildung 5.5), was eher in Einklang zu bringen wäre mit der Nicht-Verlagerung des Titan-Peaks in $<100>-$ Richtung.

Dennoch muss man insgesamt festhalten, dass eine $\beta$-Platzbesetzung zwar möglich ist, es aber dann zu einem stark unterschiedlichen Evaporationsverhalten und einer unterschiedlichen Peakverschiebung für verschiedene Richtungen kommt. Ebenfalls ist eine $\alpha$-Platzbesetzung schlüssig, dann müsste es zu einer starken Peakverschiebung des Titans kommen. 


\subsubsection{Simulationen der Messungen}

Für $\mathrm{Fe}_{3} \mathrm{Al}-\mathrm{Ti}$ sind die Schlussfolgerungen anhand der Messungen bezüglich der Platzbesetzung also nicht so eindeutig wie bei den anderen Legierungen. Daher ist gerade hier die Simulation von Interesse.

Für das Paarpotential von Ti-Ti gibt es eine Vielzahl an Literaturwerten, einige allerdings nur mit modifizierten EAM-Potentialen [72, 81, $88,95,96]$. Das Minimum wird dabei stets mit ca. $3 \AA$ angegeben (orientiert an dem NNAbstand der Titan-Gleichgewichtsphase von

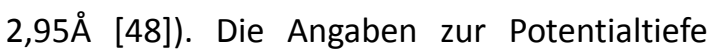
schwanken zwischen $0,21 \mathrm{eV}$ und $0,58 \mathrm{eV}$.

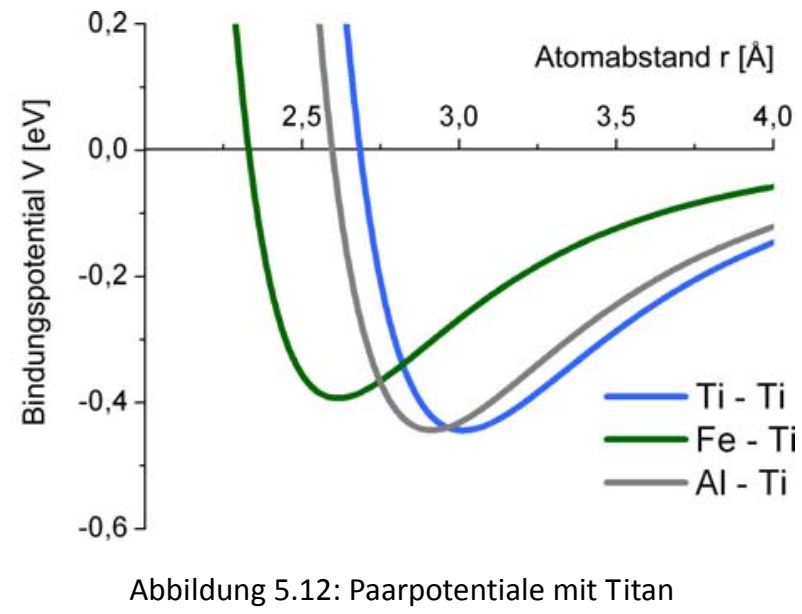

Ein Fe-Ti-Potential wird nur in einer Quelle genannt [97], in der ein modifiziertes EAM-Modell verwendet wird, so dass deren Werte nur eingeschränkt Beachtung finden können. Die Potentialtiefe hat demnach einen Wert, der ungefähr denen der reinen Fe-Fe- bzw. Ti-Ti-Potentialen entspricht. Die Posi-

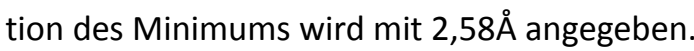

Für das Al-Ti-Potential können in der Literatur mehrere Angaben gefunden werden [81, 88, 96, 98]. Die Werte für die Potentialtiefe schwanken dabei innerhalb von 0,22eV bis 0,55eV; die Lage des Potential-

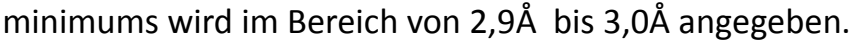

Um zunächst eine $\beta$-Platzbesetzung zu simulieren, wurden die Potentiale im Gültigkeitsbereich der oben beschriebenen Literaturquellen so angepasst, die Deckungsgleichheit von Simulationen und Messungen (Abbildung 5.11) zu maximieren. Die daraus resultierenden Paarpotentiale zeigt Abbildung 5.12. Die Dichteprofile der simulierten APT-Messungen sind in Abbildung 5.13 dargestellt.

In der $<100>-$ Richtung ist zu erkennen, dass die Titan-Peaks relativ zu den Al-Peaks eine nur geringe Verschiebung von ca. 0,15Å zeigen. In <111>-Richtung hingegen zeigen sie eine starke Verlagerung relativ zu ihrer erwarteten Position, die mittig zwischen den Al-Peaks liegt. Sie sind deutlich nach links verschoben und überlagern die Aluminium-Peaks annähernd. Ein Abstand zwischen Ti- und Al-Peaks von ca. 0,3Å ist jeweils zu erkennen, dies entspricht relativ gut der Messung, bei der dieser Abstand ca. $0,25 \AA ̊$ beträgt. Das Dichteprofil der <110>-Richtung zeigt eine leichte Verschiebung des Titan-Peaks nach links um ca. 0,2Å. Dieser Wert ist damit etwas stärker ausgeprägt als in der entsprechenden Messung (ca. 0,1̊). 

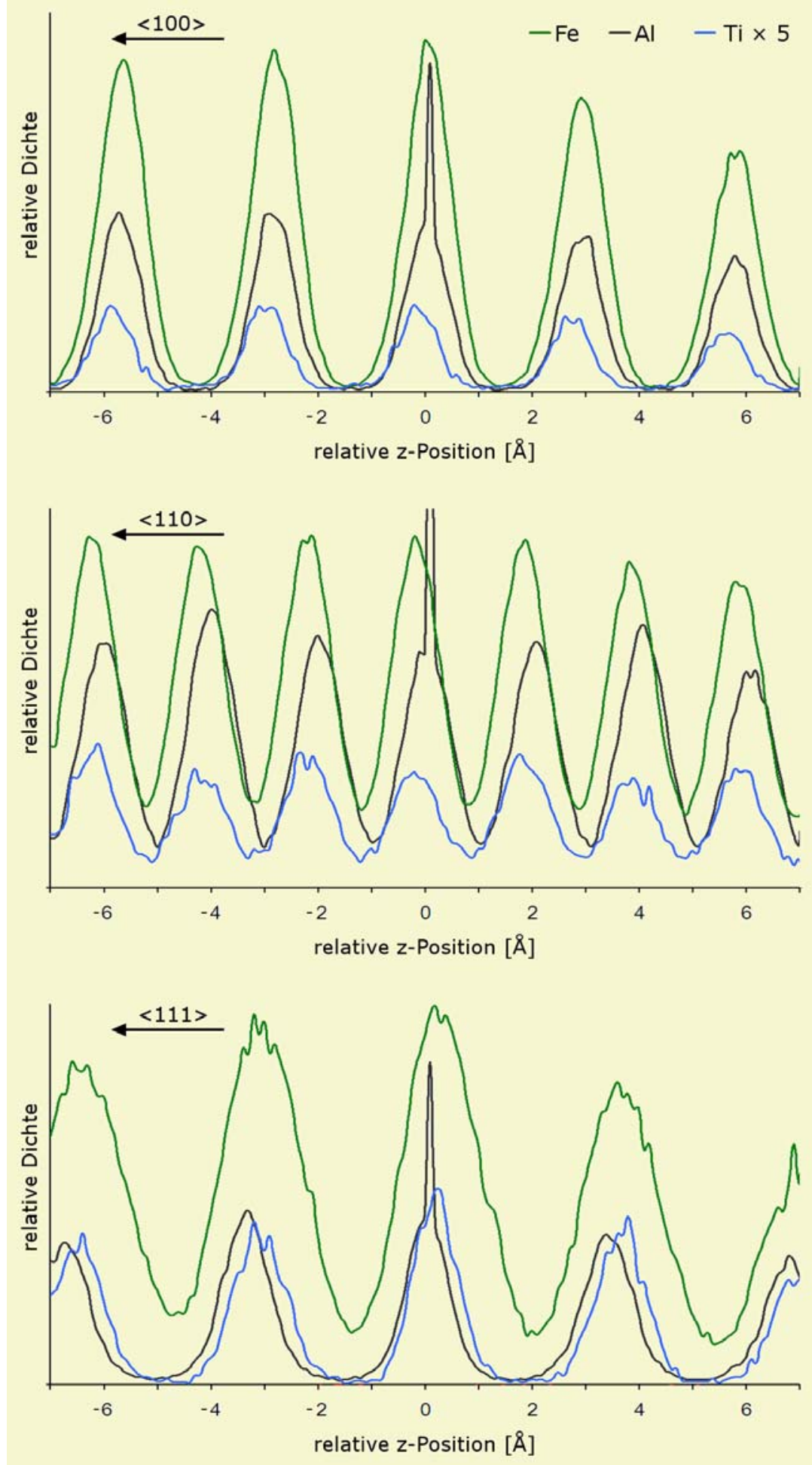

Abbildung 5.13: AtomVicinity-Dichteprofile simulierter APT-Messungen von $\mathrm{Fe}_{3} \mathrm{Al}-\mathrm{Ti}$ mit $\beta$-Platzbesetzung

Insgesamt können also die gemessenen Dichteprofile mit einer $\beta$-Platzbesetzung des Titans durch realistische Paarpotentiale simuliert werden. Die daraus ermittelten Ordnungsgrade sind $\xi_{A, Y}=0,92 \pm 0,04$ bzw. $\xi_{\mathrm{Ti}, \beta}=0,90 \pm 0,06$. Um unstrittige Aussagen über die Ordnung zu treffen, muss dennoch die ebenfalls mögliche $\alpha$-Platzbesetzung untersucht werden. Dafür müssen wiederum die Paarpotentiale auf maximale Deckungsgleichheit von Messung und Simulation angepasst werden. Als Ti-Ti- bzw. Fe-TiPotentiale wurden diejenigen aus Abbildung 5.12 verwendet. Die Simulationen sollen an dieser Stelle 
zweimal mit unterschiedlichen Al-TiPotentialen durchgeführt werden, die sich in ihren Positionen des Minimums unterscheiden, wie nebenstehend in Abbildung 5.14 dargestellt.

Die entsprechenden AtomVicinityDichteprofile sind in Abbildung 5.15 illustriert. Für das Potential mit $r_{0}=2,7 \AA$ erkennt man, dass es in $<100>$ - und $<111>$-Richtung zu einer deutlichen Peakverschiebung des Titans nach links kommt. Dadurch überlagern die Ti-Peaks die Al-Peaks und entspre-

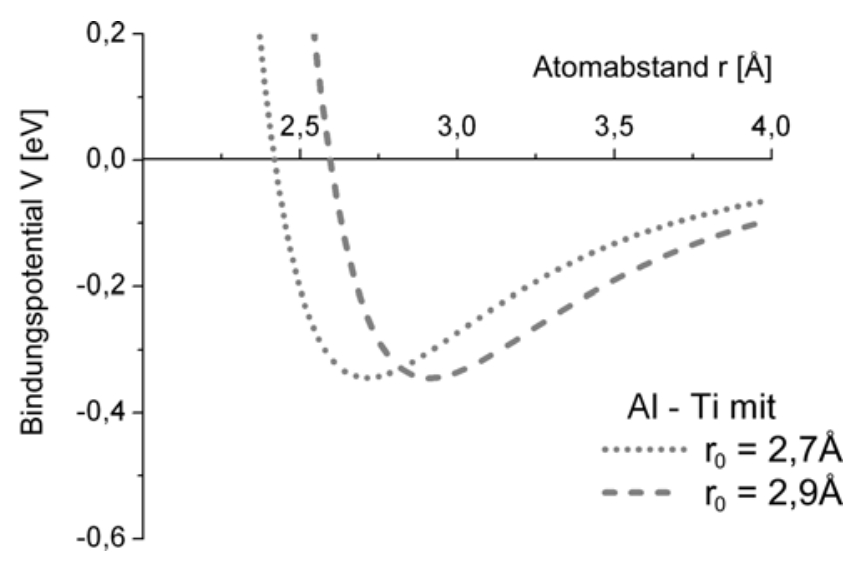

Abbildung 5.14: Al-Ti-Paarpotential mit zwei unterschiedlichen Positionen des Minimums chen damit den gemessenen Dichteprofilen (Abbildung 5.11) relativ gut. Etwas anders ist die Situation für die <110>-Orientierung, hier ist der Titan-Peak ein deutliches Stück nach links verschoben. Zwar ist diese Verlagerung nicht so stark ausgeprägt wie in den anderen Richtungen und die Peaks einer Ebene sind immer noch als zusammengehörend zu erkennen, allerdings sind die Verlagerungen in $\langle 110\rangle$ - Richtung bei anderen Legierungen jeweils deutlich weniger stark ausgeprägt. Vergleicht man in diesem Kontext dieses Profil nun mit der entsprechenden Messung, in der kein Abstand zwischen Al- und Ti-Peak zu verzeichnen ist, erscheint das simulierte Profil nur bedingt übereinstimmend.

Besonderes Augenmerk soll hier auf das Al-Ti-Potential gelegt werden. Dieses hat in den oben beschriebenen Simulationen ein Energieminimum bei $r_{0}=2,7 \AA \AA$. Verwendet man jedoch einen größeren Wert von $r_{0}$, der den Literaturangaben entspricht, hat dies zur Folge, dass Aluminium und Titan im Abstand von 2,49 (NN-Abstand dieser $\mathrm{DO}_{3}$-Struktur) anstatt einer anziehenden eine repulsive Wechselwirkung erfahren (siehe Abbildung 5.14). Dies ist aber genau der Abstand, den Al und Ti im Falle einer $\alpha$-Platzbesetzung hätten. Wie sich dieser Effekt auf die simulierten APT-Messungen auswirkt, ist ebenfalls in Abbildung 5.15 dargestellt. In $<100>$-Richtung kommt es zu einer noch stärkeren Verschiebung des Ti-Peaks, in <111>-Richtung ist diese wiederum nicht mehr so stark ausgeprägt. Gerade im letztgenannten Fall kommt es zu einer starken Diskrepanz zwischen simulierten und gemessenen Profilen.

Nun kann argumentiert werden, dass das Ti-Al-Potential im Bereich von 2,5^ ungenügend beschrieben ist. Allerdings wird der Potentialverlauf sehr ähnlich von FARKAS et al. [98], RUDA et al. [81] und BOLL [96] dargestellt, alle berechneten eine repulsive Wirkung für den Abstand von 2,49Å. Der Potentialverlauf von ZOPE und MISHIN [88] ist hingegen etwas flacher, so dass es bei $r=2,49 \AA$ noch eine sehr geringe anziehende Wirkung gibt. Dennoch erscheint es widersprüchlich, dass Aluminium- und Titanatome derart angeordnet sind, dass sie einen Abstand von 2,49尺̊ haben ( $\alpha$-Platzbesetzung). Denn im Fall einer $\beta$-Platzbesetzung wäre ihr Abstand 2,89Å, was relativ gut dem Abstand der Potentialminima aus den genannten Quellen entspricht. 

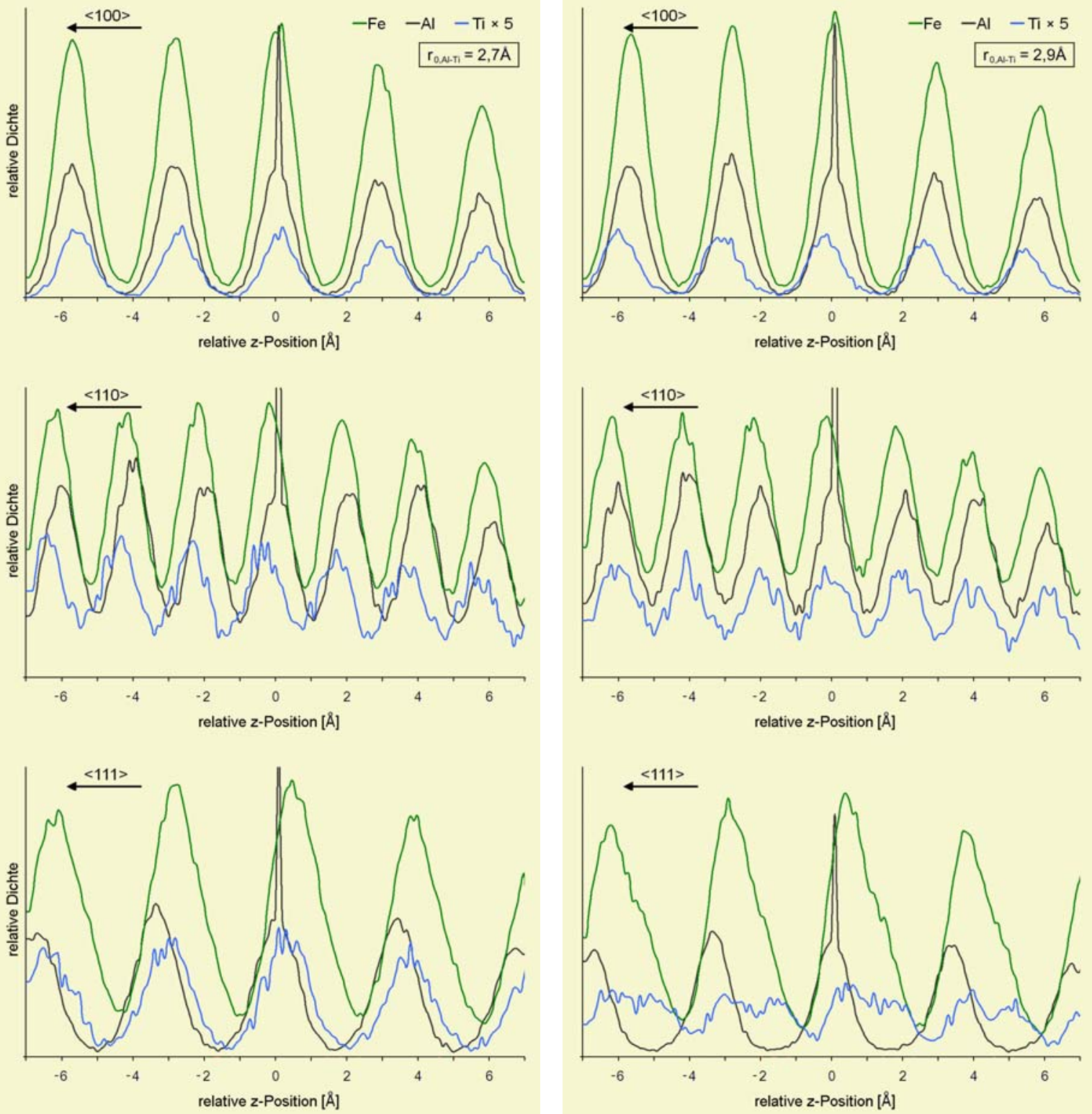

Abbildung 5.15: AtomVicinity-Profile simulierter APT-Messungen an $\mathrm{Fe}_{3} \mathrm{Al}-\mathrm{Ti}$ mit $\alpha$-Platzbesetzung für $\mathrm{r}_{0, \mathrm{Al}-\mathrm{Ti}}=2,7 \AA$ (links) und $\mathrm{r}_{0, \mathrm{Al}-\mathrm{Ti}}=2,9 \AA$ (rechts)

Zusammenfassend kann also gesagt werden, dass $\mathrm{Fe}_{3} \mathrm{Al}-\mathrm{Ti}$ mit $\alpha$-Platzbesetzung nur mit dem kürzeren Al-Ti-Potential zu Simulationen führt, deren Dichteprofile mit den gemessenen im Wesentlichen übereinstimmen. Weder entspricht dieses Potential den Literaturangaben, noch sind dann die Simulationen der <110>-Richtung uneingeschränkt konsistent. Verwendet man ein Al-Ti-Potential, das den Literaturangaben entspricht, können die gemessenen Dichteprofile nicht zufriedenstellend simuliert werden, gerade in $<111>$-Orientierung.

Damit erscheint die $\alpha$-Platzbesetzung als unwahrscheinlich und eine $\beta$-Platzbesetzung stellt die konsistenteste Ordnung dar. Allerdings muss festgehalten werden, dass dieses Ergebnis nicht so eindeutig ist wie für die anderen Legierungen. 


\section{Kapitel 6: ALCHEMI-Messungen an massivem $\mathrm{Fe}_{3} \mathrm{Al}$}

Zur vertieften Bestimmung der Platzbesetzung, sind ergänzend als Vergleichsstudie ALCHEMI-Messungen durchgeführt worden. Diese Analysen und die entsprechenden Untersuchungsmethoden werden im Folgenden vorgestellt.

\subsection{Anpassung der Auswertungsmethoden}

Für die Zusätze Chrom, Mangan und Vanadium wurden ALCHEMI-Messungen mit dem Zweistrahlmessverfahren durchgeführt, die im Prinzip nach Formel 3.19 ausgewertet werden können. Allerdings ergibt sich dabei die besondere Situation, dass Eisen zwei verschiedene Kristallplätze besetzt. Damit gibt es für die drei Plätze $\alpha, \beta$ und $\gamma$ nur zwei EDX-Signale (von Eisen und von Aluminium), so dass man grundlegend eine Information zu wenig hat. Daher kann man nur zwischen zwei verschiedenen Kristallpositionen unterscheiden. Eine entsprechende Messung ist möglich, wenn als Einstrahlrichtung die $<100>$ Zonenachse gewählt wird und die Verkippung in Richtung eines <001>-Pols erfolgt. Die Ebenenabfolge (entsprechend der Grafik links aus Abbildung 4.5) ist dann eine gemischte Ebene ( $\beta$ - und $\gamma$-Plätze) und eine reine Eisenebene ( $\alpha$-Plätze).

Es sind also nur die $\alpha$-Plätze von den anderen zu unterscheiden, eine Auszeichnung zwischen $\beta$ - und -Plätzen ist nicht möglich. Weiterhin muss beachtet werden, dass in beiden Ebenen Eisen vorhanden ist, welches zusammen das entsprechende EDX-Signal des Eisens bildet. Eine gegenseitige Annullierung der Intensitätsveränderung beider Einstrahlrichtungen findet nur deshalb nicht statt, weil die Eisenkonzentration in der gemischten Ebene ( $\beta$-Plätze) nur halb so hoch wie die der reinen Fe-Ebene ist ( $\alpha$-Plätze). Dennoch muss Formel 3.19 entsprechend angepasst werden. Für die Intensitäten gilt nunmehr analog zu Formel 3.18:

$$
\begin{aligned}
& \mathrm{I}_{\mathrm{Fe}}=\mathrm{k} \cdot \mathrm{C}_{\mathrm{Fe}} \cdot\left(\mathrm{j}_{\mathrm{A}}+\frac{\mathrm{j}_{\mathrm{B}}}{2}\right) \quad \mathrm{I}_{\mathrm{Al}}=\mathrm{k} \cdot \mathrm{C}_{\mathrm{Al}} \cdot \frac{\mathrm{j}_{\mathrm{B}}}{2} \quad \mathrm{I}_{\mathrm{X}}=\mathrm{k} \cdot \mathrm{C}_{\mathrm{X}} \cdot \mathrm{n}_{\mathrm{X}} \cdot\left(\xi_{\mathrm{X}, \mathrm{B}} \cdot \mathrm{j}_{\mathrm{B}}+\left(1-\xi_{\mathrm{X}, \mathrm{B}}\right) \cdot \mathrm{j}_{\mathrm{A}}\right) \\
& \mathrm{I}_{\mathrm{Fe}}^{\prime}=\mathrm{k}^{\prime} \cdot \mathrm{C}_{\mathrm{Fe}} \cdot\left(\mathrm{j}_{\mathrm{A}}^{\prime}+\frac{\mathrm{j}_{\mathrm{B}}^{\prime}}{2}\right) \quad \mathrm{I}_{\mathrm{Al}}^{\prime}=\mathrm{k}^{\prime} \cdot \mathrm{C}_{\mathrm{Al}} \cdot \frac{\mathrm{j}_{\mathrm{B}}^{\prime}}{2} \quad \mathrm{I}_{\mathrm{X}}^{\prime}=\mathrm{k}^{\prime} \cdot \mathrm{C}_{\mathrm{X}} \cdot \mathrm{n}_{\mathrm{X}} \cdot\left(\xi_{\mathrm{X}, \mathrm{B}} \cdot \mathrm{j}_{\mathrm{B}}^{\prime}+\left(1-\xi_{\mathrm{X}, \mathrm{B}}\right) \cdot \mathrm{j}_{\mathrm{A}}^{\prime}\right)
\end{aligned}
$$

Dabei sind k, k' Intensitätsfaktoren der Messung, C sind abermals Faktoren, die die lonisationswahrscheinlichkeiten, Fluoreszenzausbeuten und andere Skalierungsfaktoren beinhalten und $\mathrm{n}_{\mathrm{x}}$ die Anteile der Zusätze auf den jeweiligen Ebenen.

Die Doppelverhältnisbildung ist dann geringfügig diffiziler, da $\mathrm{I}_{\mathrm{Fe}}$ sowohl von $\mathrm{j}_{\mathrm{A}}$ als auch von $\mathrm{j}_{\mathrm{B}}$ abhängt. Der Grad der Platzbesetzung $\xi_{X, B}$ von X-Atomen auf B-Plätzen, also $\beta$ - und $\gamma$-Plätzen, ist demzufolge:

$$
\xi_{\mathrm{X}, \mathrm{B}}=\frac{\frac{2}{3} \cdot \frac{\mathrm{I}_{\mathrm{X}}}{\mathrm{I}_{\mathrm{X}}^{\prime}}-\frac{\mathrm{I}_{\mathrm{Fe}}}{\mathrm{I}_{\mathrm{Fe}}^{\prime}}+\frac{1}{3} \cdot \frac{\mathrm{I}_{\mathrm{Al}}}{\mathrm{I}_{\mathrm{Al}}^{\prime}}}{\frac{\mathrm{I}_{\mathrm{Al}}}{\mathrm{I}_{\mathrm{Al}}^{\prime}}-\frac{\mathrm{I}_{\mathrm{Fe}}}{\mathrm{I}_{\mathrm{Fe}}^{\prime}}}
$$




\subsection{Messergebnisse}

Die Messungen wurden an der Ohio State University, Columbus (USA) an einem Tecnai F20 Transmissionselektronenmikroskop der Firma FEI durchgeführt. Alle Messungen wurden bei 200kV Betriebsspannung vorgenommen, die EDX-Spektren wurden mit einem SSD-Detektor des angeschlossenen EDXSystems des Tecnai gemessen, bei jeweils 60s Messdauer.

Die Ergebnisse wurden mit der entsprechenden Anwendersoftware analysiert; dazu sind jeweils die Intensitäten der K-Linien-Übergänge betrachtet worden. Das Grundrauschsignal ist durch Anfitten einer Exponentialfunktion an die Störsignale ermittelt und subtrahiert worden. Die Peaks selbst wurden durch Summation der Ereignisse in einem Intervall um den Peak ermittelt. Durch Anfitten einer Lorentzfunktion konnte eine Fehlerabschätzung erfolgen.

Für die gemessenen Legierungen wurden jeweils mehrere Messungen beider Richtungen (Channeling/Random) durchgeführt. Zur Analyse sind diejenigen ausgewählt worden, bei denen das Verhältnis $\mathrm{I}_{\mathrm{A}} / \mathrm{I}_{\mathrm{X}}$ die stärksten Unterschiede aufwies, da in diesem Fall anzunehmen ist, dass der Channelingeffekt am wenigsten gestört wurde. Diese Messdaten sind im Anhang (B.4) zu finden. Die Ergebnisse für einen $\beta$ - $/ \gamma$-Platzbesetzungsgrad $\xi_{B}$ lauten:

\begin{tabular}{ccc}
\hline Chrom & Mangan & Vanadium \\
\hline$\xi_{\mathrm{Cr}, \mathrm{B}}=0,90 \pm 0,19$ & $\xi_{\mathrm{Mn}, \mathrm{B}}=0,75 \pm 0,52$ & $\xi_{\mathrm{V}, \mathrm{B}}=1,02 \pm 0,29$ \\
\hline
\end{tabular}

Tabelle 6.1: Messergebnisse der ALCHEMI-Messungen (Zweistrahlverfahren)

Der Messfehler wurde anhand der Bestimmtheit der Intensitäten durch Fehlerfortpflanzung ermittelt. Für alle betrachteten Elemente kann eine $\beta$-Platzbesetzung erkannt werden. Bei Mangan ist diese Aussage jedoch aufgrund des schwach ausgeprägten Channeligeffekts und der daraus folgenden großen relativen Messungenauigkeit eine nur sehr unpräzise Aussage möglich.

Für die titanhaltige Probe konnte keine Analyse erfolgen, da bei den entsprechenden Messungen kein Channelingeffekt zu beobachten war. Der Grund dafür kann eine zu hohe Defektdichte sein, diese Aspekte werden in 8.3.1 diskutiert.

Eine weitere ALCHEMI-Messreihe, bei der der kippwinkelabhängige Intensitätsverlauf mit theoretischen Berechnungen verglichen wurde, führte leider ebenfalls zu keinen verwertbaren Ergebnissen. Diese Messungen werden als Ergänzung in Anhang A vorgestellt. 


\section{Kapitel 7: Messungen an nanokristallinem ternären $\mathrm{Fe}_{3} \mathrm{Al}$}

Die nanokristallinen Proben werden nun durch TEM- und insbesondere durch APTMessungen untersucht. Durch systematische Analysen und statistische werden Fragen der Homogenität, Segregation und Komposition anhand der APT-Daten begutachtet. Des Weiteren sollen aber auch die Schwierigkeiten genannt sein, die APT-Messungen verkomplizieren.

\subsection{TEM-Messungen}

Die für APT-Messungen angefertigten Proben wurden zunächst im Transmissionselektronenmikroskop (TEM) untersucht. Da die APT-Spitzen im vorderen Bereich eine Dicke von unter 100nm zeigen, sind sie für TEM-Untersuchungen geeignet und es mussten keine speziellen TEM-Proben hergestellt werden.

Die Hellfeldaufnahmen zeigen anhand des unterschiedlichen Kontrasts, dass es sich um eine nanokristalline Struktur handelt. Dunkle Bereiche werden dabei neben Beugung am Kristallgitter einzelner Körner auch durch Streuung an Versetzungen, Korngrenzen und anderen Defekten verursacht.

Um die Korngröße und -struktur zu untersuchen, sind Dunkelfeldaufnahmen daher geeigneter. In Abbildung 7.1 sind diese von einer APT-Spitze von $\mathrm{Fe}_{3} \mathrm{Al}-4$ at\% $\mathrm{Cr}$ (Probencharge 3) für drei verschiedene Beugungsreflexe dargestellt. Deutlich sind einzelne Körner zu erkennen. Ihre Verteilung ist derart dünn, dass eine visuelle Überlagerung (zwei hintereinander liegende Körner erscheinen als eines) relativ unwahrscheinlich ist. Folglich lassen sich die Korngrößen gut messen, was umgesetzt wurde, indem ein als Korn erkannter heller Bereich jeweils in senkrechter und waagerechter Ausdehnung vermessen wurde.

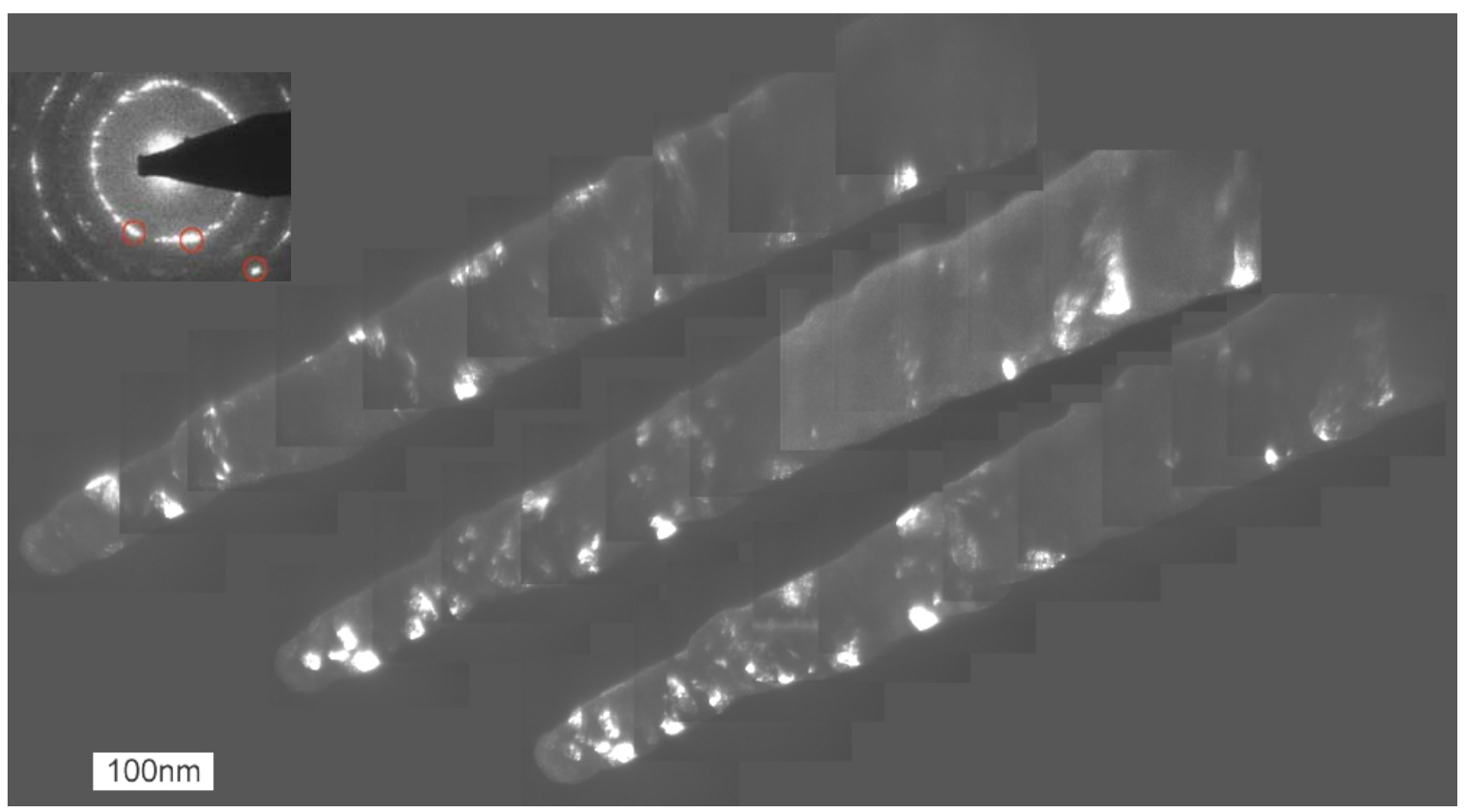

Abbildung 7.1: Dunkelfeld-TEM-Aufnahmen von $\mathrm{Fe}_{3} \mathrm{Al}-\mathrm{Cr}$ (Charge 3)

(Bildercollage mehrerer Einzelaufnahmen, Beugungsreflexe und Blendenbereich sind oben links markiert) 
Die durchschnittlichen Korngrößen sind in Tabelle 7.1 aufgelistet. Es zeigt sich, dass für alle Kompositionen Korngrößen im Wesentlichen zwischen 4nm und 30nm gefunden werden. Der Durchschnitt liegt jeweils bei etwa $10 \mathrm{~nm} \pm 6 \mathrm{~nm}$. Diese Angabe der Ungenauigkeit resultiert aus der großen statistischen Schwankung, die Messungenauigkeit von ca. 1,5nm ist dabei eher von geringerer Bedeutung. Diese Ergebnisse bestätigen die Resultate der XRD-Untersuchungen (siehe 3.2.2 und [60])

\begin{tabular}{ccc}
\hline \multicolumn{2}{c}{ Probe } & $\begin{array}{c}\text { Korngröße [nm] } \\
\text { Chargen-Nr. }\end{array}$ \\
\hline 1 & Legierung & (mit Angabe der Standardabweichung) \\
2 & $\mathrm{Fe}_{3} \mathrm{Al}-\mathrm{Cr}$ (MA) & $8,1 \pm 4,0$ \\
3 & $\mathrm{Fe}_{3} \mathrm{Al}-\mathrm{Ti}(\mathrm{MA})$ & $9,4 \pm 6,9$ \\
4 & $\mathrm{Fe}_{3} \mathrm{Al}-\mathrm{Cr}$ & $8,8 \pm 4,9$ \\
5 & $\mathrm{Fe}_{3} \mathrm{Al}-\mathrm{Mn}$ & $10,1 \pm 5,3$ \\
6 & $\mathrm{Fe}_{3} \mathrm{Al}-\mathrm{V}$ & $11,5 \pm 6,5$ \\
\hline
\end{tabular}

Tabelle 7.1: Körngrößen ermittelt aus TEM-Untersuchungen

In den TEM-Bildern ist keine Anisotropie in der Gefügestruktur zu erkennen. Auch anhand der Beugungsbilder ist eine derartige Eigenschaft nicht zu verzeichnen, Abbildung 7.2 zeigt eine solche Aufnahme von $\mathrm{Fe}_{3} \mathrm{Al}-\mathrm{Ti}$ (Charge 6). Die Reflexe können eindeutig den Ebenen der A2-geordneten Kristallstruktur zugeordnet werden (siehe auch 2.1). Dieses Ergebnis entspricht ebenfalls den XRD-Messungen in Abschnitt 3.2.2 (Abbildung 3.4).

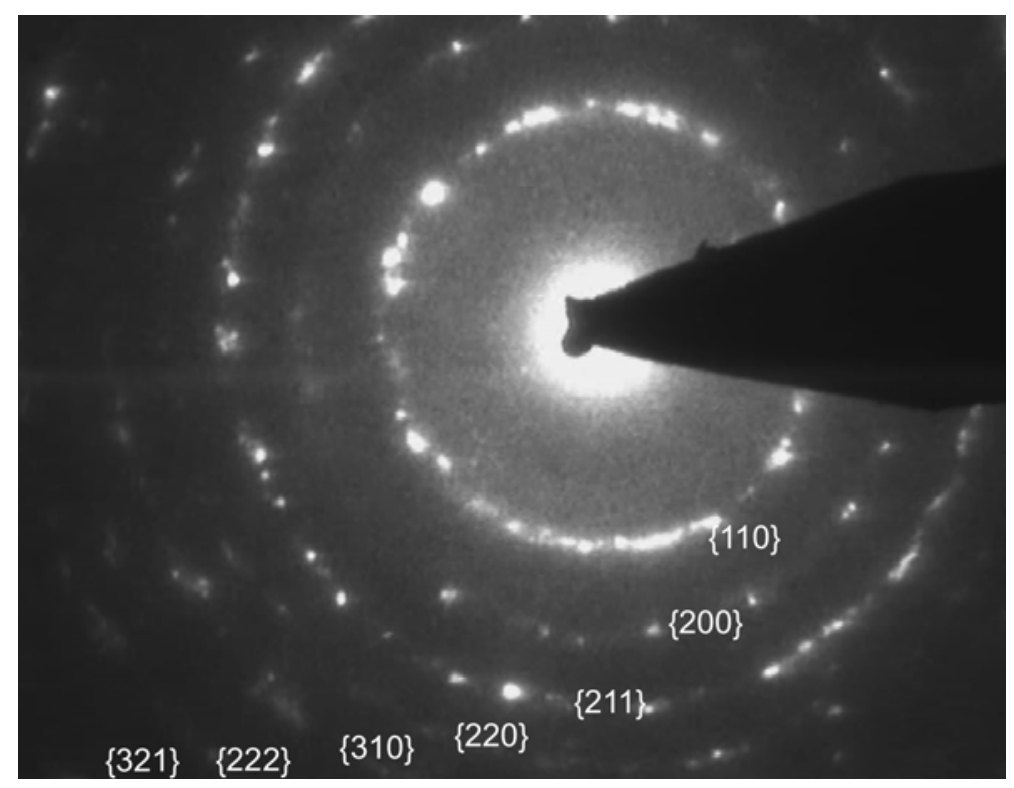

Abbildung 7.2: TEM-Beugungsbild von nanokristallinem $\mathrm{Fe}_{3} \mathrm{Al}-\mathrm{Ti}$ (Charge 6) Beugungsreflexe der A2-Ordnung sind gekennzeichnet 


\subsection{Allgemeine Betrachtungen der APT-Messungen}

Zunächst ist anzumerken, dass erfolgreiche APT-Messungen erst nach langer Erfahrungssuche zum Umgang und Präparation des Materials gelang. Da die untersuchten Legierungen relativ spröde sind, ist eine APT-Messung im Allgemeinen schwierig, weil es leicht zum Abbruch von Spitzen während der Messung kommt. Grund dafür sind die mechanischen Belastungen, die durch das anliegende elektrische Feld entstehen. Gerade nanokristallines Material ist zusätzlich anfälliger für einen Abbruch der Spitzen, da es durch die erhöhte Korngrenzen- und Defektdichte zu einem vermehrten Auftreten von Risswachstum kommen kann.

Diese Umstände führten dazu, dass nur zwei Messungen erfolgreich an der Atomsonde mit Spannungsanregung durchgeführt werden konnten, bei der die Proben durch die zusätzliche periodische Belastung häufiger abreißen. Eine solche Messung gelang am IMP der Universität Göttingen (siehe 7.3), eine andere an einem LA-WATAP der Firma Cameca an der King Abdullah University of Science and Technology (KAUST) in Saudi-Arabien.

Erfolgreichere Messungen konnten an der KAUST an einem LEAP (Local Electrode Atom Probe - Nahelektroden-Atomsonde) der Firma Cameca (vormals Imago) durchgeführt werden. An diesem Gerät können auch Messungen mit Laseranregung vorgenommen werden, bei der die mechanische Belastung der Probe sinkt. Die besondere Bauart besteht darin, dass sich die Gegenelektrode relativ nah

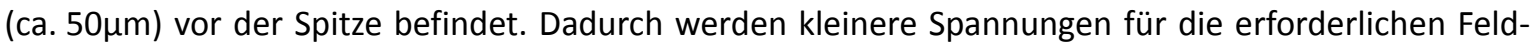
stärken benötigt, wodurch die Energieunschärfe der lonen sinkt und damit die Massenauflösung steigt [99]. Des Weiteren besitzt diese Atomsonde einen größeren Detektor, der einen größeren Erfassungswinkel der lonen zulässt und damit ein lateral größeres Volumen.

Doch auch die Präparation mit dem FIB (siehe 3.2.2) hat entscheidenden Einfluss darauf, ob eine erfolgreiche Messung durchgeführt werden kann. Wie Abbildung 7.3b zeigt, kann es zum Abbrechen der Spitze kommen oder auch zum Ablösen des gesamten Probenblocks von der Wolframspitze.

Daher musste auch der Prozess der Probenanfertigung optimiert werden:

- So wurde z.B. auf ein genaueres, geraderes Abschneiden der Wolframspitze geachtet, um die Kontaktfläche zu vergrößern (siehe Abbildung 3.5e). Dazu wurden besonders kleine Ströme (30pA) verwendet.

- Außerdem sind an jeder Seite zwei laterale Schlitze statt nur einem verwendet worden, um den Block zu fixieren (Abbildung 3.5h). Diese wurden teilweise kreuzend positioniert, teilweise nebeneinander liegend mit einem gewissen Winkel zueinander, um die Stabilität zu erhöhen.

- Um ein Abbrechen der vorderen Probenspitze zu vermeiden (Abbildung 7.3a), wurde das finale Anspitzen der Probe verändert: Ursprünglich erfolgte dieses durch einen kreisförmigen lonenstrahl von oben [53]. Dadurch entstehen aber direkt um die Spitze Einschnitte, die die Stabilität des vorderen Bereiches herabsetzen. Zusätzlich ist dieser anfälliger für Schwingungen, die zum Abbrechen führen können. Daher wurde die Methode dahingehend geändert, dass nur durch einen lonenstrahl von der Seite Material abgetragen wird, wie in Abbildung 3.5 beschrieben. 

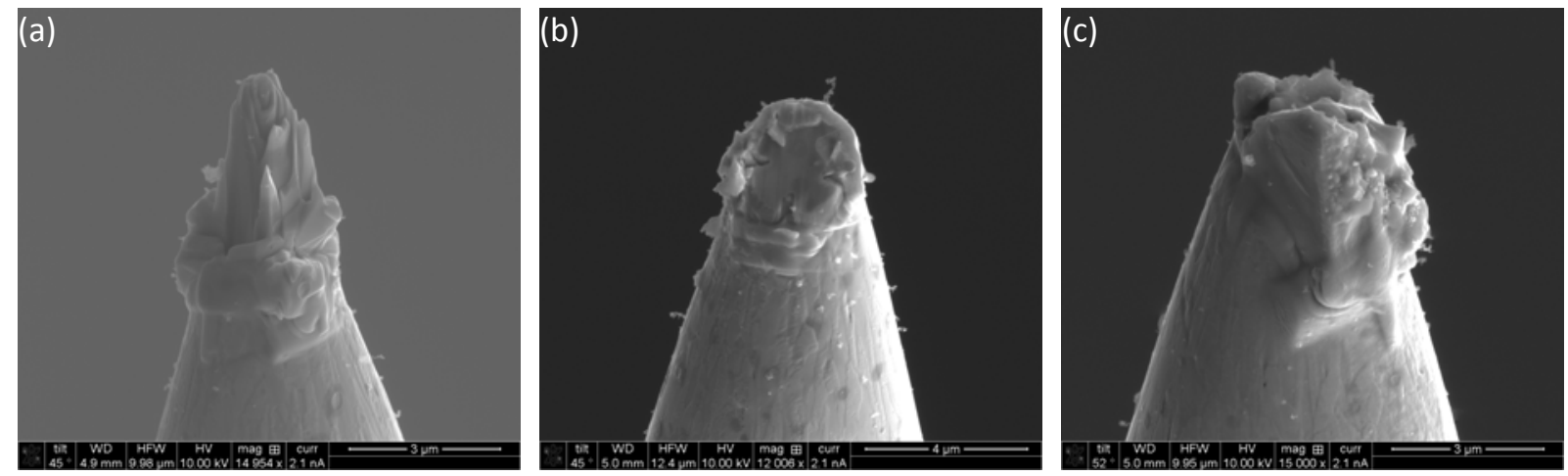

Abbildung 7.3: REM-Aufnahmen von APT-Spitzen nach erfolglosen Messungen:

(a) Abbruch des vordersten Spitzenbereiches, (b) Abbruch des gesamten Probenblocks,

(c) Verformung des gesamten Blocks, möglicherweise durch Funkenüberschlag

\subsection{APT-Analyse an $\mathrm{Fe}_{3} \mathrm{Al}-\mathrm{Cr}$, mechanisch legiert}

Die Darstellung der APT-Messung ist in Abbildung $7.4 \mathrm{zu}$ sehen, sowohl insgesamt als auch nach Elementen separiert. Für die Messung konnte kein Bereich ausfindig gemacht werden, in dem Ebenen zu identifizieren sind. Diese Tatsache erschwert eine genaue Rekonstruktion, so dass auf Erfahrungswerte zurückgegriffen werden musste; als Rekonstruktionsfaktor $k_{f}$ wurde ein Wert von 5 angenommen. Dass keine Ebenen erkannt werden konnten, liegt zum einen daran, dass bei dem nanokristallinem Material nicht gewährleistet werden kann, dass Ebenen parallel zur Messrichtung positioniert werden (also anders als bei den Messungen des massiven Materials). Stattdessen wurde die Probe in der Position gemessen, in der sie vorlag. Zum anderen liegt das auch an prinzipiellen Feldevaporationseigenschaften von nanokristallinem Material. Dieser Aspekt wird in 8.4.2 diskutiert.

Neben den Legierungselementen sind signifikant Kohlenstoff und Sauerstoff detektiert worden. Beide sind mutmaßlich herstellungsbedingt, gerade bei Kohlenstoff ist zu vermuten, dass er aus den Stahlkugeln der Mühle und/oder dem Attritor stammt.

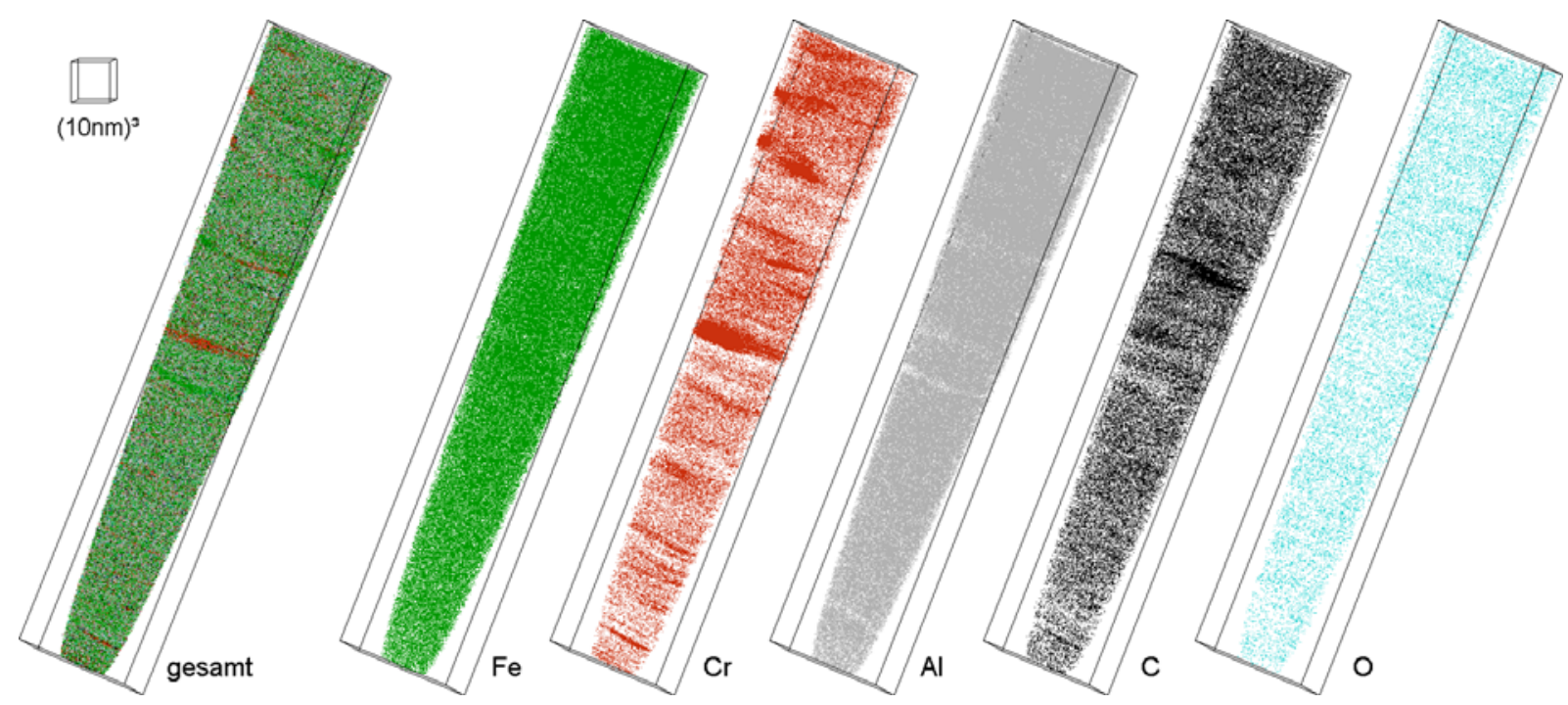

Abbildung 7.4: APT-Rekonstruktion von nanokristallinem $\mathrm{Fe}_{3} \mathrm{Al}-\mathrm{Cr}$ (Charge 1)

In den Rekonstruktionen (Abbildung 7.4) sind augenscheinlich Inhomogenitäten zu erkennen. Jedoch ist keine nanokristalline Struktur derart auszumachen, dass einzelne Körner klar von einander zu trennen sind. Weder durch Segregation noch durch Dichteschwankungen können Korngrenzen eindeutig 
bestimmt werden, jedoch sind streifenförmige Bereiche erhöhter Dichte eines Elementes zu erkennen, die annähernd parallel zueinander liegen. Genauere quantitative Betrachtungen dazu können durch Konzentrationsmessungen geschehen. Abbildung 7.5 zeigt dazu exemplarisch die elementaren Konzentrationsverläufe entlang einer mittig aus dem Messvolumen extrahierten Säule mit 8nm² Grundfläche. Die Gesamtkonzentrationen und Segregationsindizes der beteiligten Elemente sind in Tabelle 7.2 verzeichnet.

In dem Konzentrationsprofil und hierbei gerade im linken Teil der Grafik ist zu erkennen, dass Eisen und Aluminium stellenweise gleichmäßig im Bereich der statistischen Varianz verteilt sind. An anderen Punkten schwankt deren Konzentration jedoch sehr stark, wie in Abbildung 7.4 bereits zu erkennen ist. Bei Eisen z.B. treten Spitzen von 35at\% bis 94at\% auf, dagegen fällt die Aluminiumkonzentration bisweilen auf bis zu 5at\% ab. Dargestellt sind auch die $2 \sigma-G r e n z e n$ der Konzentrationsverteilung von Eisen und Aluminium (siehe 3.1.2). Es liegen jeweils $80 \%$ bzw. $81 \%$ der Messpunkte innerhalb dieses Bereiches und entsprechen damit nicht den Kriterien einer homogenen Verteilung nach Formel 3.4.

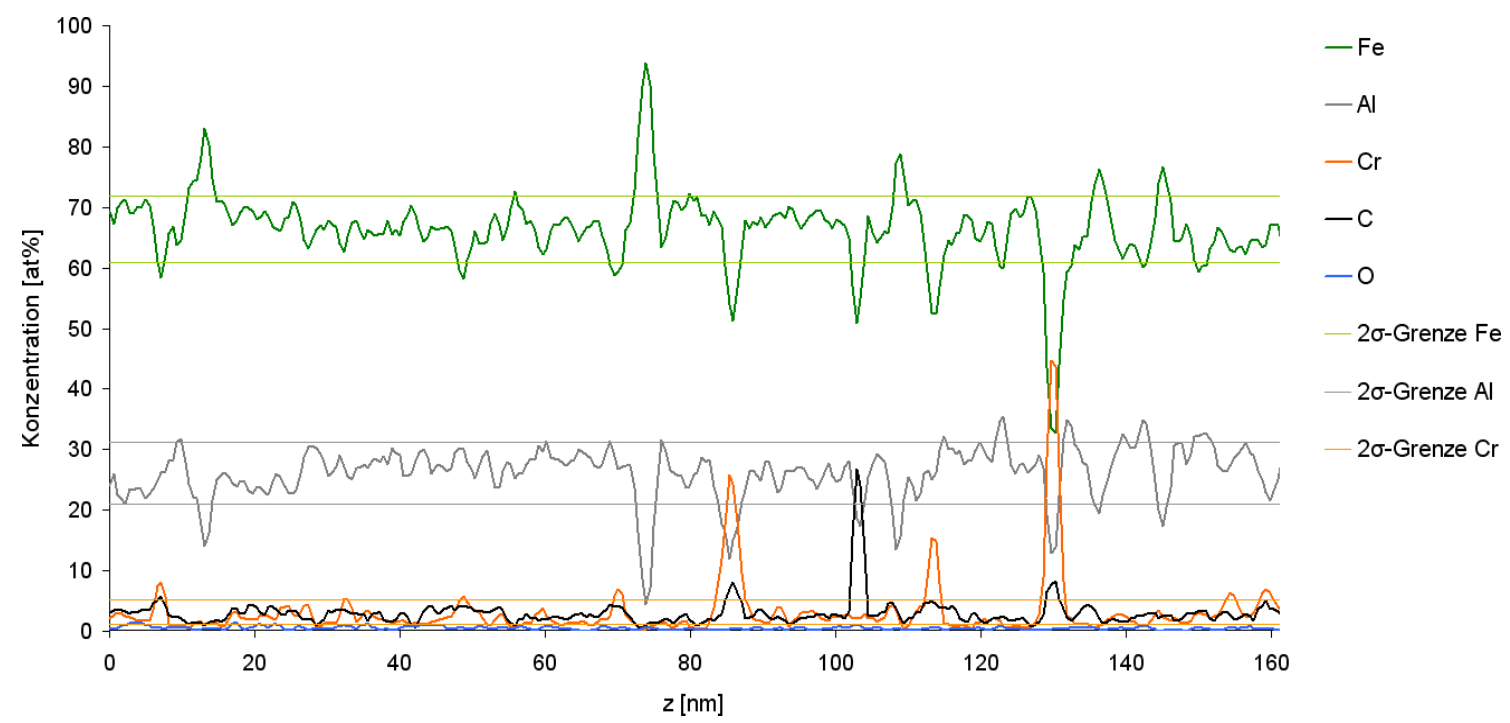

Abbildung 7.5: Konzentrationsverläufe von nanokristallinem $\mathrm{Fe}_{3} \mathrm{Al}-\mathrm{Cr}$

(Charge 1), Analysevolumen mit $8 \mathrm{~nm}^{2}$ Grundfläche

Ebenfalls sind bei Chrom-Peaks von bis zu annähernd 50at\% zu erkennen. Hier fällt zudem auf, dass eine erhöhte Chromkonzentration oft mit einer überdurchschnittlich hohen Kohlenstoffkonzentration korreliert. Daneben gibt es aber auch Bereiche, in denen nur Kohlenstoff-Peaks zu sehen sind. Der Segregationsparameter (Tabelle 7.2) bestätigt diese Beobachtungen. Er ist besonders hoch bei Chrom, bei dem ca. 30\% der Atome mehr über dem Konzentrationsmittelwert liegen, als es bei homogener Verteilung der Fall wäre. Auch für Kohlenstoff, Eisen und Aluminium ist der Segregationsparameter erhöht,

\begin{tabular}{lcccccc}
\hline & & $\mathrm{Fe}$ & $\mathrm{Al}$ & $\mathrm{Cr}$ & $\mathrm{C}$ & $\mathrm{O}$ \\
\hline Konzentration [at\%] & $\overline{\mathrm{C}}$ & 66,6 & 26,4 & 3,3 & 3,3 & 0,6 \\
& nominell & 69 & 26 & 5 & 0 & 0 \\
\hline Segregationsindex [\%] & $\mathrm{H}$ & 3,6 & 7,9 & 42,7 & 23,3 & 33,2 \\
& $\mathrm{H}_{\text {hom }}$ & 1,7 & 4,1 & 13,3 & 13,5 & 30,4 \\
\hline
\end{tabular}

Tabelle 7.2: Quantitative Analyse von nanokristallinem $\mathrm{Fe}_{3} \mathrm{Al}-\mathrm{Cr}$ (Charge 1) 
was wiederum zum Ausdruck bringt, dass keine vollkommene homogene Verteilung dieser Elemente vorliegt.

Bei der genauen Definition von Homogenität ist allerdings zu beachten, dass es sich um ein nanokristallines Gefüge handelt. Damit ist der Anteil, der nah an Korngrenzen liegt, relativ hoch. Veränderte Konzentrationen im Bereich von Korngrenzen sind daher von intergranularer Inhomogenität zu unterscheiden. Diese Aspekte werden in 8.5 diskutiert.

Das Konzentrationsprofil zeigt an mehreren Stellen ein gleichlaufendes Ansteigen der Chrom- und Kohlenstoffkonzentration. Um derartige Korrelationen noch genauer zu charakterisieren, wird eine Kreuzkorrelationsanalyse nach 3.1.2 durchgeführt (Abbildung 7.6).

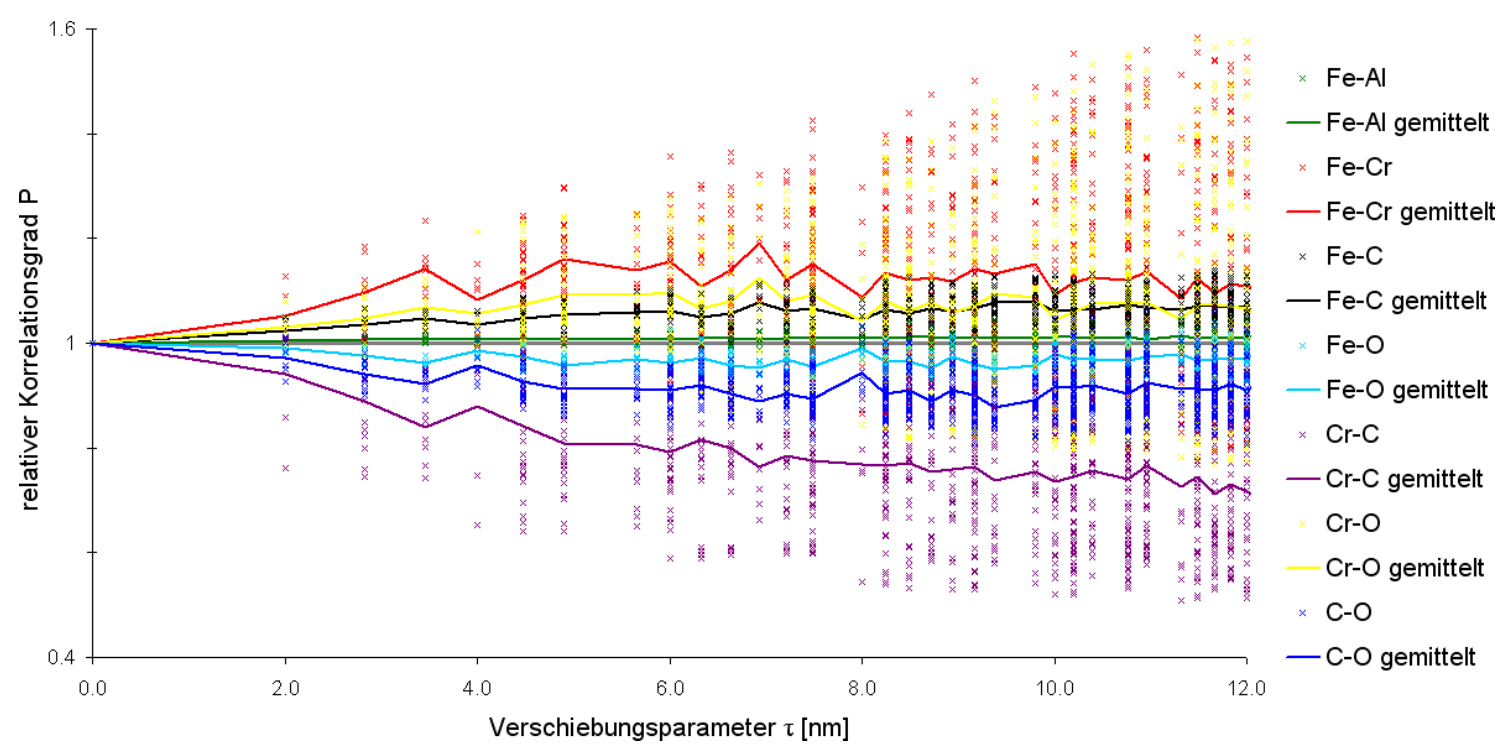

Abbildung 7.6: Kreuzkorrelationsanalyse an nanokristallinem $\mathrm{Fe}_{3} \mathrm{Al}-\mathrm{Cr}$

Betrachtet man für $\mathrm{Cr}$ und Fe die Korrelation mit Kohlenstoff, so kann ein unterschiedliches Verhalten festgestellt werden. $P_{\mathrm{Cr}, \mathrm{c}}$ sinkt für steigendes $\tau$ signifikant, so dass von einem zusammenhängenden Auftreten von Chrom und Kohlenstoff auszugehen ist. $\mathrm{P}_{\mathrm{Fe}, \mathrm{C}}$ hingegen bleibt stets leicht über dem Wert von 1, hier kann eine leichte Segregation von Eisen und Kohlenstoff erkannt werden. Das Absinken von $\mathrm{P}_{\mathrm{C}, \mathrm{O}}$ zeigt, dass die kohlenstoffreicheren Bereiche erhöhte Sauerstoffanteile aufzeigen.

Eisen und Aluminium zeigen keine Korrelation, der Wert $\mathrm{P}_{\mathrm{Fe}, \mathrm{Al}}$ bleibt relativ konstant bei 1 und weicht nur im Rahmen statistischer Schwankung davon ab. Gleiches gilt für die übrigen Korrelationsparameter.

Einen interessanten Aspekt zeigt der Verlauf von $\mathrm{P}_{\mathrm{Fe}, \mathrm{Cr}}$. Der Wert steigt zunächst signifikant für größer werdendes $\tau$, was eine entmischende Tendenz von Eisen und Chrom repräsentiert. Ab einem gewissen Punkt bei ca. $\tau=7 \mathrm{~nm}$ treten jedoch auch wieder Werte auf, die unter 1 liegen, auch wenn der gemittelte Wert weiterhin über 1 liegt. Im Bereich der Schwankungen kann eine Segregation nicht mehr so eindeutig festgestellt werden. Eine mögliche Erklärung dafür ist, dass Chrom vermehrt an Korngrenzen auftritt (was auch den Peaks im Konzentrationsprofil entspräche), die Eisenkonzentration wäre dort demzufolge geringer. Eine Verschiebung der Konzentrationsverläufe gegeneinander führte dann solange zu einem erhöhtem $\mathrm{P}_{\mathrm{Fe}, \mathrm{Cr}}$, bis die Verschiebung $\tau$ Werte im Bereich des Korndurchmessers annähme. Dann würden sich wiederum (an einigen Stellen) die Korngrenzen überlagern und $\mathrm{P}_{\mathrm{Fe}, \mathrm{cr}}$ wieder sinken. Da dies in Abbildung 7.6 ab $\tau \approx 7 \mathrm{~nm}$ der Fall ist, ist anzunehmen, dass dies in etwa der Durchmesser der kleinsten auftretenden Körner ist. 


\subsection{APT-Analyse an $\mathrm{Fe}_{3} \mathrm{Al}-\mathrm{Ti}$, mechanisch legiert}

Die APT-Messungen an mechanisch legiertem $\mathrm{Fe}_{3} \mathrm{Al}-\mathrm{Ti}$ wurden wie alle weiteren Proben bis auf $\mathrm{Fe}_{3} \mathrm{Al}$ $\mathrm{Mn}$ (Charge 4) an einem LEAP bei einer Laserenergie von 30pJ bei 80K durchgeführt. Da die Verwendung einer Laseranregung im Allgemeinen zu einem vermehrten Auftreten von Molekülevaporation und einer ungenaueren Massenbestimmung führt als bei Spannungsanregung, soll zunächst ein Blick auf das folgende Massenspektrum geworfen werden.
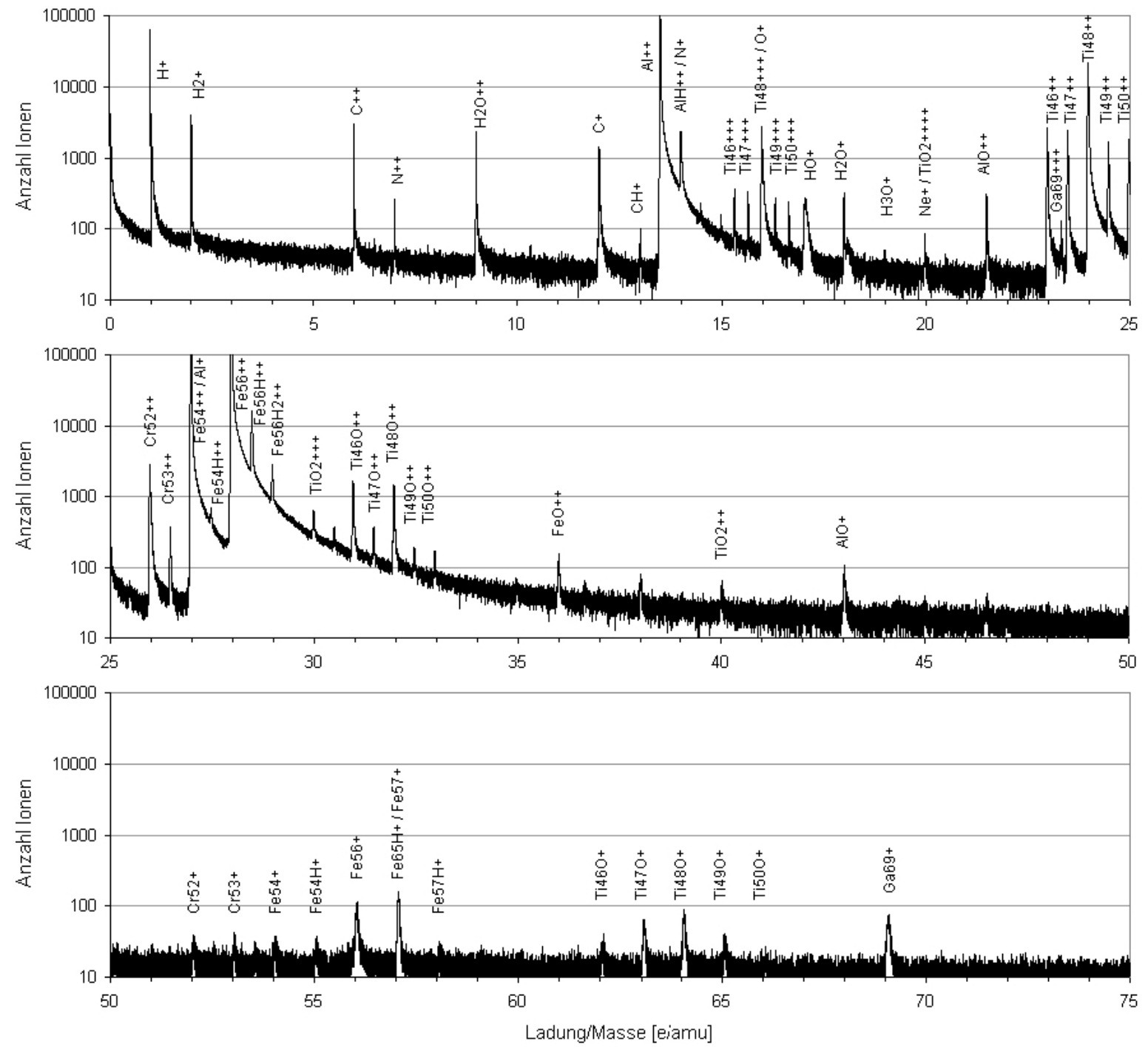

Abbildung 7.7: Massenspektrum einer APT-Messung an $\mathrm{Fe}_{3} \mathrm{Al}-\mathrm{Ti}$ (Charge 3)

Markant sind die Peaks der Hauptbestandteile Eisen, Aluminium und Titan, die jeweils relativ breit sind, mit einer ausgeprägten Schulter an der rechten Seite. Diese Tatsache erschwert die genaue Zuordnung eines Massenverhältnisses zu einem Element. Außerdem sorgt sie für eine ungenauere Konzentrationsbestimmung.

Ebenfalls ist auffällig, dass neben den erwarteten Peaks insbesondere viele Oxide zu verzeichnen sind, gerade TiO tritt vermehrt auf. In diesem Zusammenhang ist es interessant, einen Ausschnitt des rekonstruierten Volumens nach lonen getrennt zu betrachten (Abbildung 7.8). Denn dort ist jeweils eine obere „Kappe“ zu erkennen, in der ein erhöhtes Auftreten dieser Oxide, aber auch von Kohlenstoff, Gallium, Wasserstoff und dem Rauschsignal (ein Signal, dessen Massenverhältnis nicht zugeordnet werden kann) zu verzeichnen ist. Es ist anzunehmen, dass es sich dabei um eine verunreinigte Schicht 
auf der Probe handelte, die während der Probenpräparation entstanden ist, ggf. zusätzlich durch spätere Oxidation bzw. Adsorption. Der scharfe Übergang zu dem darunterliegenden Bereich könnte bedeuten, dass der letzte Teil dieser Schicht sehr plötzlich abgerissen ist.

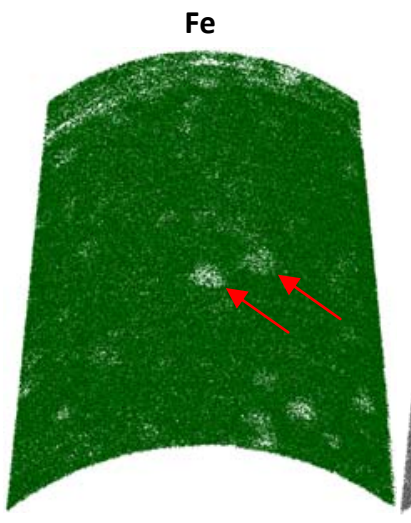

$\mathrm{FeO}$

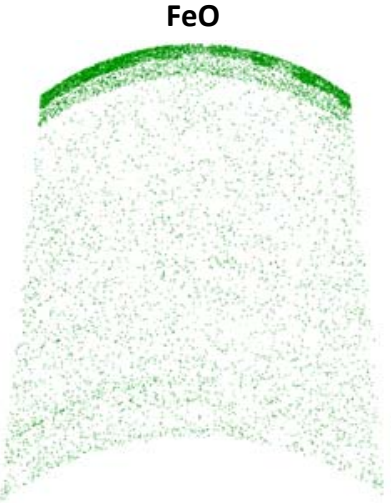

H
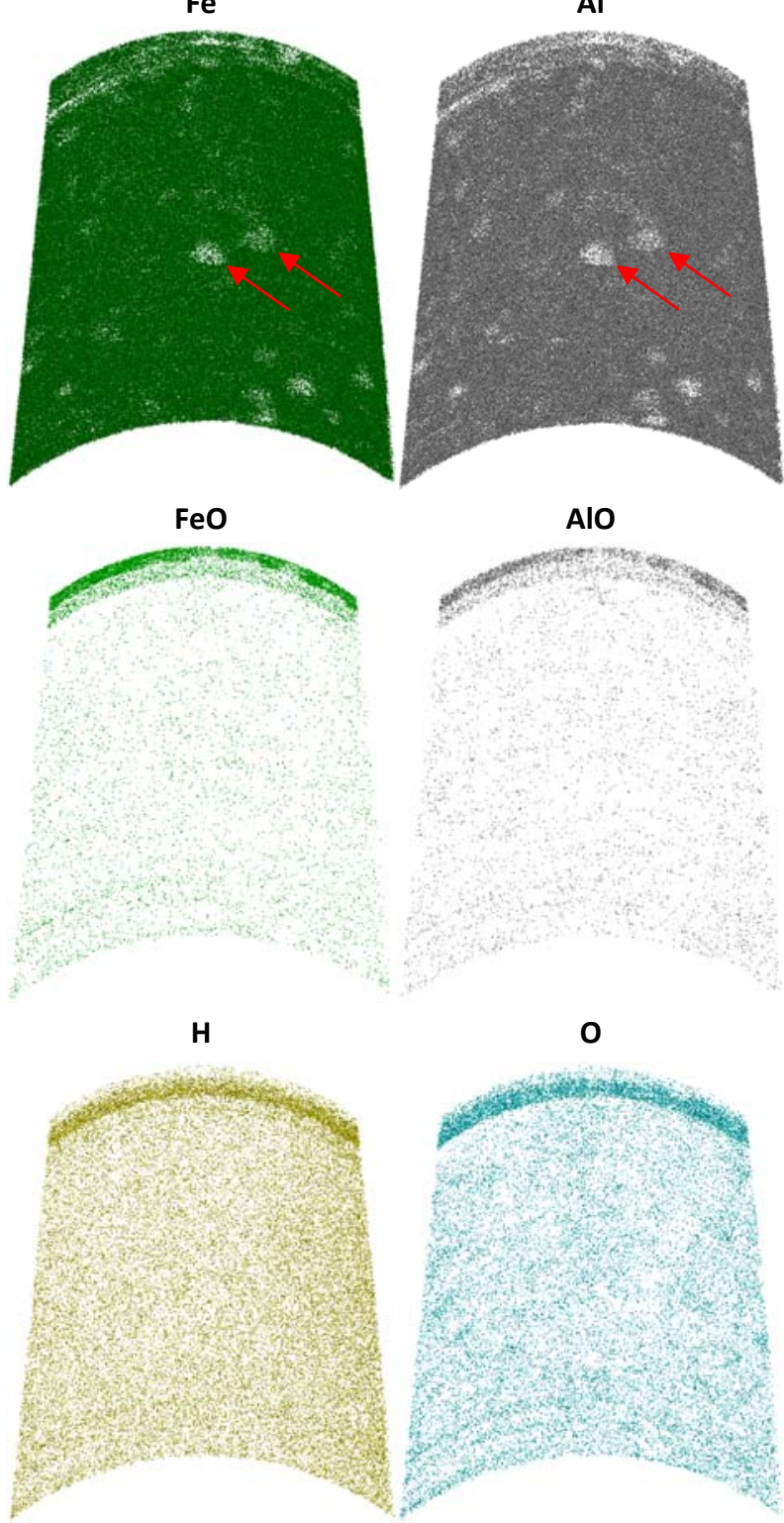

AlO

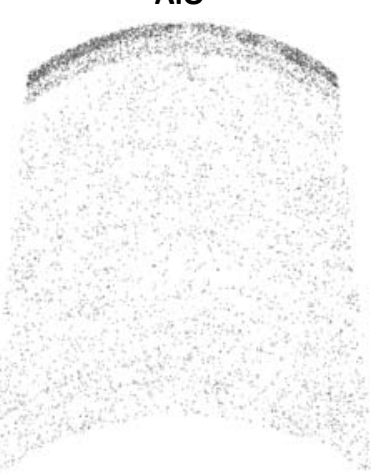

o

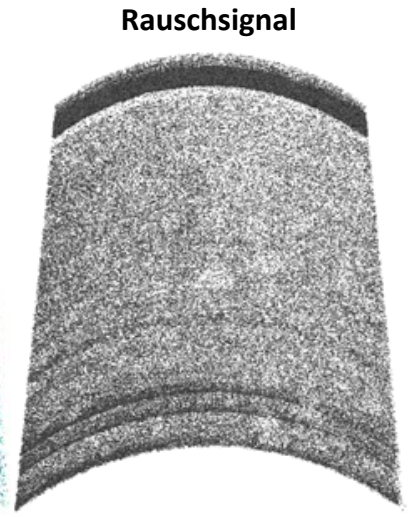

$\mathrm{Ti}$

C

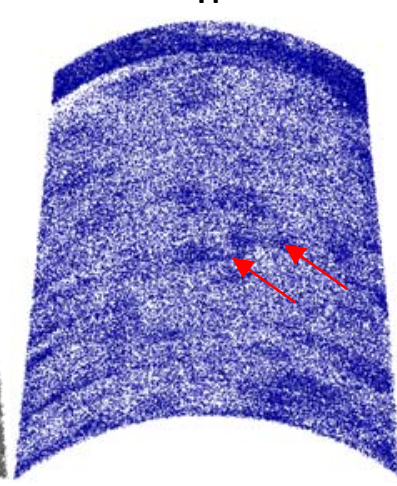

TiO

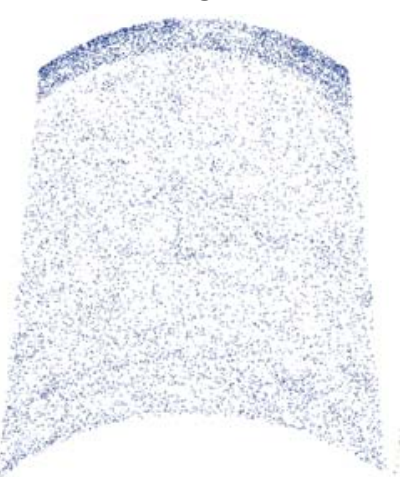

Abbildung 7.8: Nach lonen getrennte Rekonstruktion einer APT-Messung von $\mathrm{Fe}_{3} \mathrm{Al}-\mathrm{Ti}$

(zur besseren Erkennbarkeit ein Ausschnitt mit 10nm Tiefe, rote Pfeile: siehe Text)

In dem übrigen Volumen sind im Wesentlichen $\mathrm{Fe}, \mathrm{Al}, \mathrm{Ti}, \mathrm{C}$ und $\mathrm{O}$ detektiert worden. Dieser Teil des Volumens weist deutlich weniger Oxide und Gallium auf. Letzteres spricht dafür, dass die Probenpräparation mit genügend Umsicht erfolgte, so dass das Material weitestgehend unverändert blieb. In diesem Bereich sinkt auch das Rauschsignal auf ein normales Maß. Wasserstoff ist ebenfalls detektiert worden, allerdings muss dieses nicht zwingend im Material gewesen sein. Gerade Wasserstoff besitzt in UHV-Anlagen einen erhöhten Partialdruck, es wurde für die Konzentrationsberechnung daher nicht beachtet, genauso wie die obere „Kappe“ der Probe.

In dem rekonstruierten Volumen ist ebenfalls keine nanokristalline Struktur auszumachen, es sind also keine Korngrenzen durch Segregation oder Dichteschwankungen eindeutig zu erkennen. Es existieren aber Bereiche, in denen Titan und Kohlenstoff agglomeriert erscheinen, Eisen und Aluminium weisen 
dort eine geringere Dichte auf (rote Pfeile). Zur Untersuchung dieses Aspekts ist in Abbildung 7.9 ein eindimensionales Konzentrationsprofil entlang einer mittigen Säule mit einer Grundfläche von $5 \mathrm{~nm}^{2}$ dargestellt. Darin ist zum einen die obere Kappe zu erkennen, die sich durch hohe Konzentrationen an Wasserstoff, Kohlenstoff und Sauerstoff auszeichnet. Erst nach ca. 10nm sinken diese Werte auf ein Durchschnittsmaß. Zum anderen sind einzelne Bereiche zu erkennen, an denen die Titankonzentration auf bis zu 45at\% steigt, diese besitzen eine Breite von ca. 4nm. Dies bestätigt die optische Beobachtung von Titananhäufungen aus Abbildung 7.8, sie korrelieren mit einem erhöhten Auftreten von Kohlenstoff, aber auch von $\mathrm{H}$ - und O-Atomen.
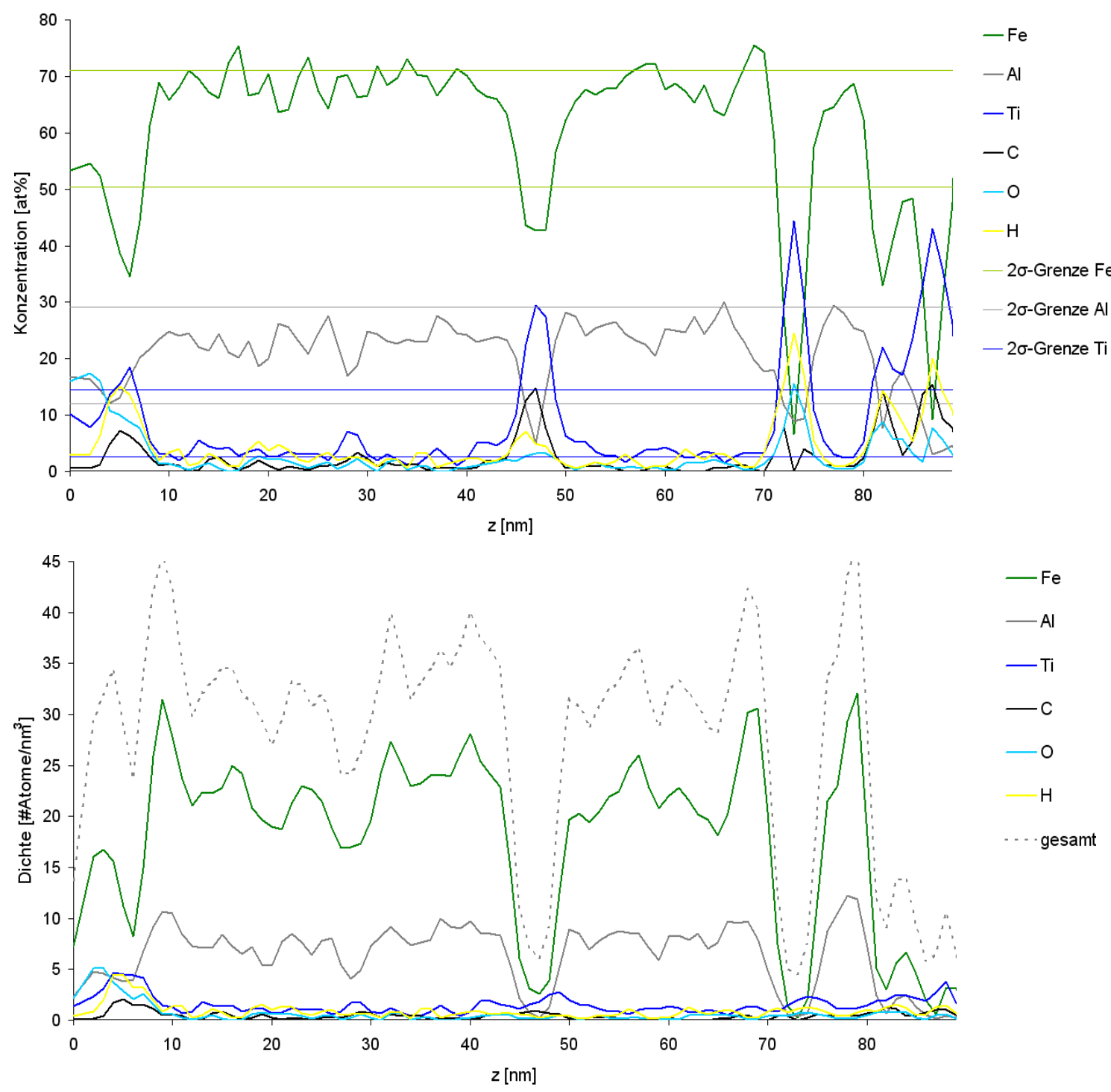

Abbildung 7.9: Eindimensionaler Konzentrations- und Dichteverlauf von $\mathrm{Fe}_{3} \mathrm{Al}-\mathrm{Ti}$ (Charge 2)

Allerdings erscheint die Konzentrationsbestimmung in einem anderen Licht, wenn man für dasselbe Analysevolumen die Dichteverläufe betrachtet. Diese sind ebenfalls in Abbildung 7.9 dargestellt. Anzumerken ist, dass die gemessenen APT-Volumina unter Berücksichtigung einer gewissen Detektionsrate (hier 37\%) rekonstruiert werden, die gemessene Dichte entspricht damit nicht der nominellen.

An Stellen der erhöhten Titankonzentration steigt die Dichte des Titans nur etwa um den Faktor zwei. Erst dadurch, dass die Gesamtdichte auf ca. ein viertel ihres Wertes sinkt, entstehen die berechneten Titankonzentrationen. Jedoch ist dieses Absinken der atomaren Gesamtdichte ein Messergebnis, das sehr unrealistisch wirkt. Auch wenn sie aufgrund unterschiedlicher Kristallstruktur und interstitiell ge- 
lösten Atomen an einigen Stellen variieren kann, so ist ein solcher Abfall nicht zu erwarten. Mann muss davon ausgehen, dass es sich um Messartefakte handelt. Eine Erklärung ist z.B., dass der Bereich mit erhöhtem Titananteil eine höhere Feldverdampfungsfeldstärke besitzt und daher während der Messung zeitweise die Evaporationsrate an dieser Stelle sinkt. In der Rekonstruktion resultiert dies in einem Bereich kleinerer Dichte, dieser Effekt ist vergleichbar mit dem „lokalen Vergrößerungseffekt" [48, 100]. Genauer wird dieser Aspekt in Kap. 8.4 diskutiert. An dieser Stelle soll zunächst festgehalten werden, dass eine absolute Konzentrationsberechnung diesem Effekt unterliegt. Somit kann nur die Erhöhung der Titandichte Anhaltspunkt für Inhomogenitäten sein.

Es sei angemerkt, dass eine solche inkonstante Dichte bei der in 7.3 beschriebenen Messung, die an einer mit Spannungsanregung arbeitenden Atomsonde durchgeführt wurde, nicht auftrat. Folglich kann es sich um ein spezifisches Problem der titanhaltigen Legierung oder der Laseranregung handeln.

Für die Berechnungen des Segregationsindexes $\mathrm{H}$ (Tabelle 7.3) werden aufgrund des Auftretens dieser Artefakte die absoluten Atomanzahlen verwendet.

\begin{tabular}{lcccccc}
\hline & & $\mathrm{Fe}$ & $\mathrm{Al}$ & $\mathrm{Ti}$ & $\mathrm{C}$ & $\mathrm{O}$ \\
\hline \multirow{2}{*}{ Konzentration [at\%] } & $\overline{\mathrm{C}}$ & 66,4 & 26,4 & 4,1 & 1,4 & 1,6 \\
& nominell & 70 & 26 & 4 & 0 & 0 \\
\hline \multirow{2}{*}{ Segregationsindex [\%] } & $\mathrm{H}$ & 2,0 & 3,5 & 19,8 & 29,7 & 23,1 \\
& $\mathrm{H}_{\text {hom }}$ & 1,8 & 4,2 & 12,0 & 20,5 & 19,6 \\
\hline
\end{tabular}

Tabelle 7.3: Quantitative Analyse von nanokristallinem Fe ${ }_{3} \mathrm{Al}-\mathrm{Ti}$ (Charge 2)

Der Segregationsparameter des Eisens $\mathrm{H}_{\mathrm{Fe}}$ liegt geringfügig über dem Wert einer homogenen Verteilung, was die Schwankungen in der Eisendichte reflektiert. Bei Titan und Kohlenstoff sind die Segregationsindizes deutlich höher als die Werte der homogenen Verteilung, bei Sauerstoff ist die geringfügige Überschreitung hingegen kein sehr deutlicher Hinweis auf eine Ungleichverteilung.

Zur weiteren Verteilungsanalyse wurde auch für diese Probe eine Kreuzkorrelation durchgeführt (Abbildung 7.10). Insgesamt zeigt diese Probe deutlich geringere Segregationstendenzen, die Werte des Korrelationsparameters $P$ liegen jeweils nahe bei 1 . Es ist eine gewisse Korrelation zwischen Titan, Kohlenstoff und Sauerstoff zu verzeichnen, die jeweiligen Werte von $\mathrm{P}$ sinken für ein steigendes $\tau$ geringfügig.

Vergleicht man die Elemente mit Eisen, so zeigt $\mathrm{P}_{\mathrm{Fe}, \mathrm{Al}}$, dass Eisen und Aluminium eine leichte Affinität besitzen. Bei $P_{\mathrm{Fe}, \mathrm{Ti}}$ ist ein ungefähr konstanter Wert von 1 zu erkennen, d.h. Ti und Fe zeigen weder eine Tendenz zur Segregation noch eine zur Korrelation. Da $\mathrm{P}_{\mathrm{Fe}, \mathrm{C}}$ geringfügig über 1 steigt, ist bei diesen Elementen eine geringe Segregation zu verzeichnen. 


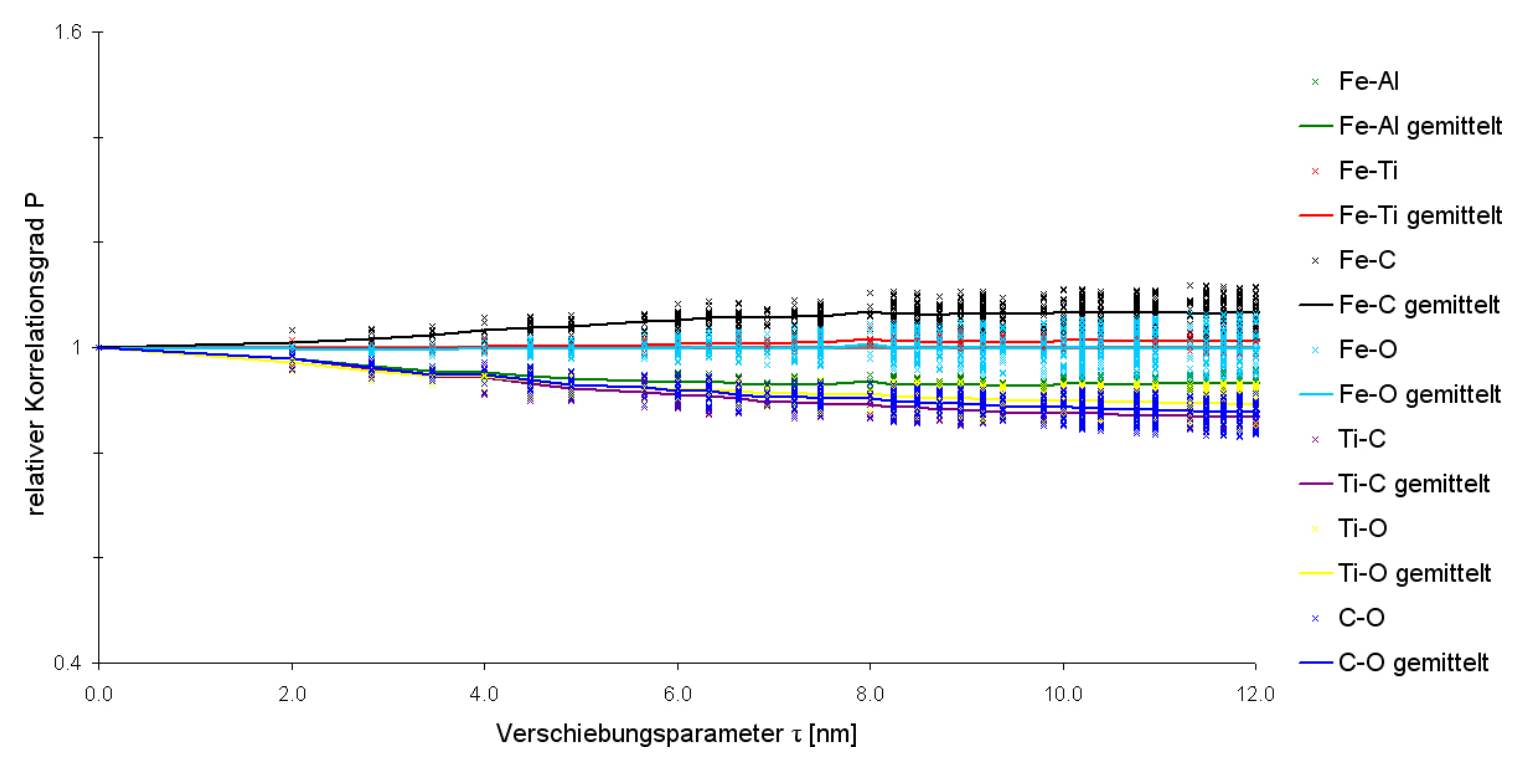

Abbildung 7.10: Kreuzkorrelationsanalyse von nanokristallinem $\mathrm{Fe}_{3} \mathrm{Al}-\mathrm{Ti}$ (Charge 2)

\subsection{APT-Analyse an $\mathrm{Fe}_{3} \mathrm{Al}-\mathrm{Cr}$, vorlegiert}

Auch in den APT-Rekonstruktionen von nanokristallinem, vor dem Kugelmahlen eingeschmolzenem $\mathrm{Fe}_{3} \mathrm{Al}-\mathrm{Cr}$ (Probencharge 3) sind keine Ebenen zu erkennen (siehe Abbildung 7.11). Ebenso ist keine nanokristalline Struktur anhand von Segregationen auszumachen, stattdessen ist ein sehr homogenes Gefüge zu erkennen, das sich deutlich von denen der mechanisch legierten Proben abhebt.

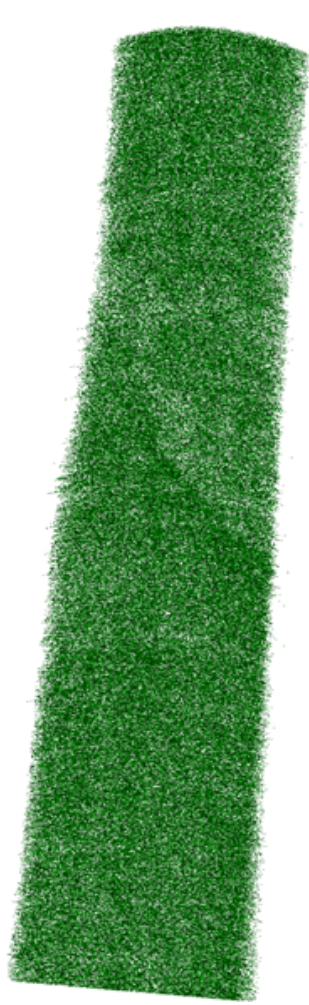

$\mathrm{Fe}$

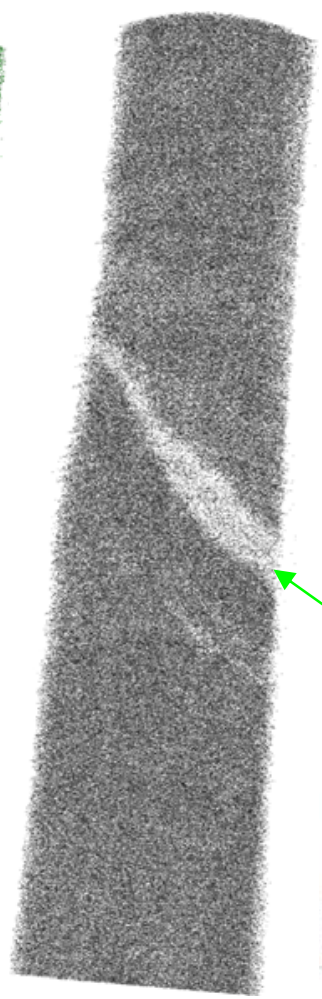

Al

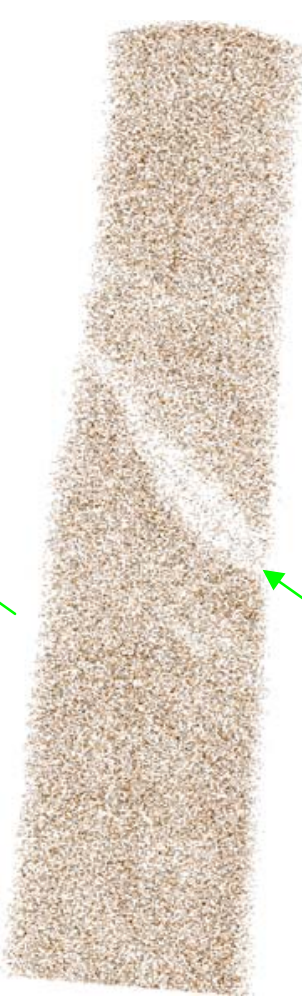

$\mathrm{Cr}$

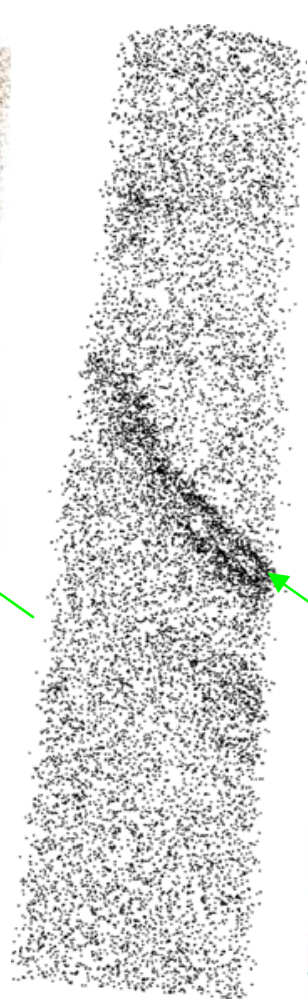

C

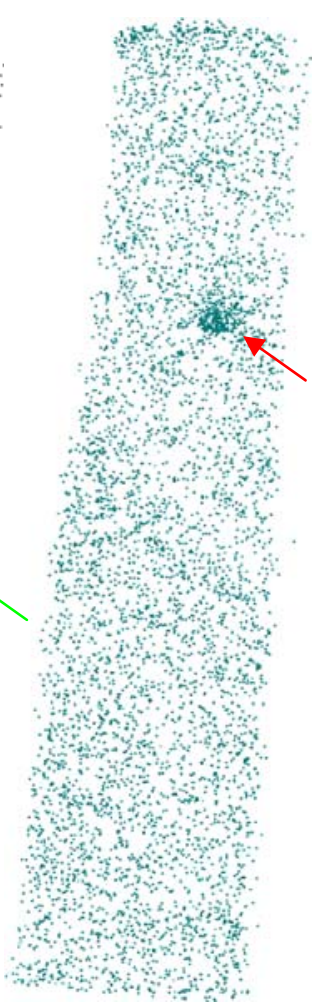

O $\square(10 \mathrm{~nm})^{3}$

Abbildung 7.11: Ausschnitte aus rekonstruiertem APT-Volumen von $\mathrm{Fe}_{3} \mathrm{Al}-\mathrm{Cr}$ (Charge 3)

(Ausschnitt von 10nm Tiefe, Pfeile: siehe Text) 
Es sind in diesen Abbildungen zwei Besonderheiten zu erkennen: Eine kleine Oxidagglomeration (roter Pfeil) sowie eine längliche Anreicherung von Kohlenstoff, verknüpft mit einer Verringerung von Chrom und Aluminium (grüne Pfeile).

Zur genaueren Analyse zeigt Abbildung 7.12 ein Dichteprofil durch diese zwei Artefakte. Bei der Oxidanreicherung (roter Pfeil) kann ein Durchmesser von 9nm gemessen werden, die Sauerstoffdichte steigt dabei auf das Zwanzigfache des durchschnittlichen Wertes. Es sind keine Veränderungen in der Dichte der anderen beteiligten Elemente zu verzeichnen. Das Entstehen einer solchen Sauerstoffanreicherung ist nicht direkt zu erklären. Prinzipiell ist nicht auszuschließen, dass es sich um ein Messartefakt handelt. So könnte dort z.B. eine Pore im Material vorhanden sein, in der während der Messung Oxide oder Wasser adsorbierten, die bei fortschreitender Feldevaporation dissoziierten und dann ebenfalls detektiert werden würden. Es kann sich aber auch um eine Oxidausscheidung handeln, die sich während des Herstellungsverfahrens bildete, z.B. aufgrund der Einarbeitung einer sauerstoffhaltigen Verunreinigung.

Die Kohlenstoffanreicherung (grüner Pfeil) weist eine Erhöhung der C-Dichte etwa um das Zehnfache auf. Hier ist eindeutig zu erkennen, dass die Dichte an Chrom und Aluminium auf bis zu 10\% absinkt. Die Eisendichte hingegen scheint sich nicht zu verändern, sondern minimal zu steigen. Dieses Gebiet zeigt also eine markant andere Zusammensatzung als das restliche Gefüge. Das Auftreten dieses kohlenstoffreichen Streifens könnte mit Abrieb von den Stahlkugeln der Mühle, aus denen der gesamte Kohlenstoff mutmaßlich stammt, erklärt werden. Eventuell ist dieser Bereich erst kurz vor Beendigung des Mahlvorgangs verunreinigt worden, so dass noch keine ausreichende Homogenisierung erfolgen konnte. Das Vorhandensein von geringen Mengen an Aluminium und Chrom in diesem Bereich kann dann durch Diffusion begründet werden.

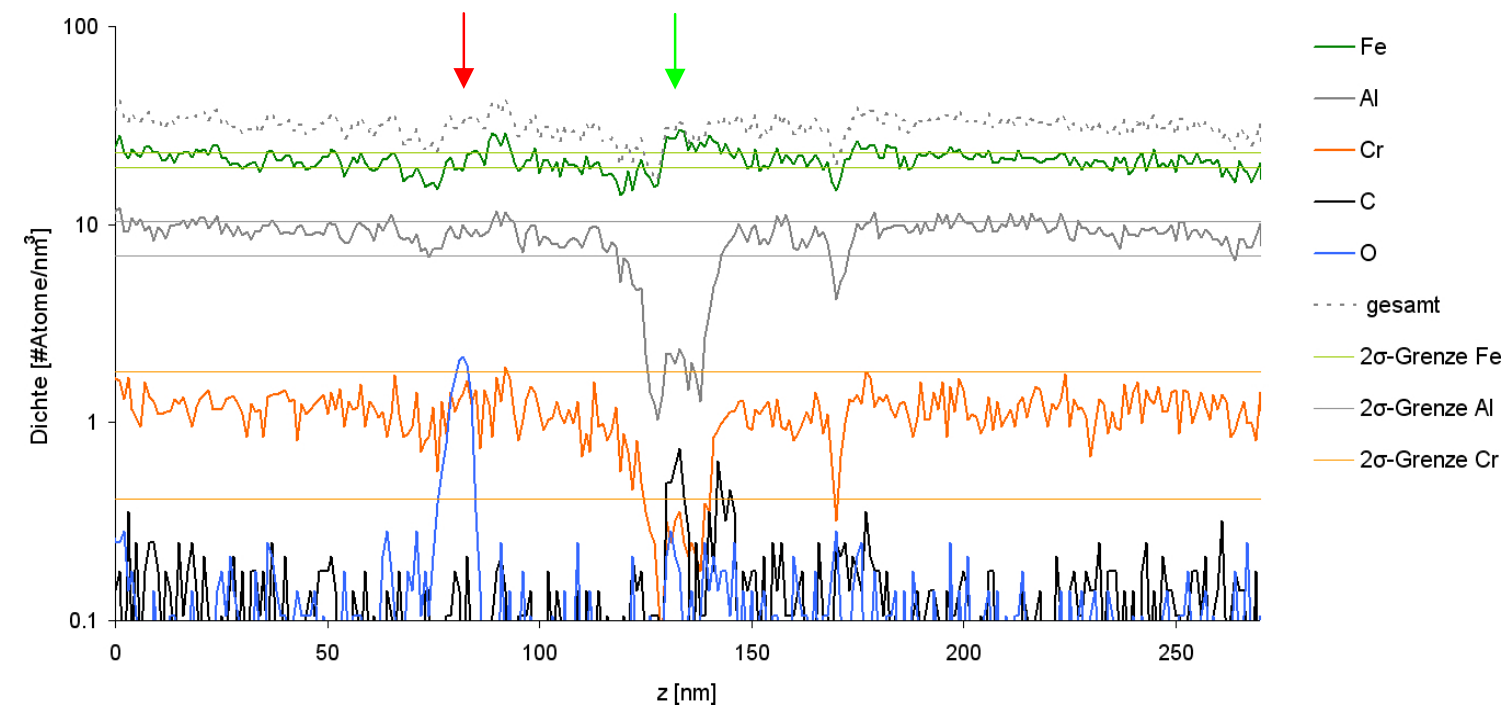

Abbildung 7.12: Eindimensionaler Dichteverlauf in nanokristallinem $\mathrm{Fe}_{3} \mathrm{Al}-\mathrm{Cr}$ (Charge 3)

Insgesamt kann diese Probe - abgesehen von den zwei beschriebenen Artefakten - als homogen bezeichnet werden. In den Rekonstruktionen und im Dichteprofil können wenige Unregelmäßigkeiten festgestellt werden, was sogar zu der Vermutung führen könnte, dass keine nanokristalline Struktur (mehr) vorliegt. Dass diese aber in der Tat gegeben ist, zeigen die TEM-Aufnahmen, die hier untersuchte Probe ist in Abbildung 7.1 dargestellt.

Ein weiteres Merkmal für die Qualität der Probe ist der geringe Grad an Fremdatomen (siehe Tabelle 7.4). Des Weiteren ist auch die konstante Dichte der APT-Messungen zu beachten, dies ist wahrschein- 
lich ebenfalls das Resultat der allgemein homogenen Struktur, die zu einer gleichmäßigen Feldevaporation und damit zu schwächeren Rekonstruktionsfehlern führt (siehe auch 8.4.2).

Die Homogenität dieser Probe unterstreichen auch die Segregationsindizes (Tabelle 7.4), welche für alle betrachteten Elemente unterhalb des Wertes $\mathrm{H}_{\text {hom }}$ einer homogenen Verteilung liegen. Das gilt auch für Kohlen- und Sauerstoff, wobei bei diesen Elementen die Konzentrationen derart niedrig sind, dass ihr Segregationsindex nur geringere Aussagekraft aufweist.

\begin{tabular}{lcccccc}
\hline & & $\mathrm{Fe}$ & $\mathrm{Al}$ & $\mathrm{Cr}$ & $\mathrm{C}$ & $\mathrm{O}$ \\
\hline \multirow{2}{*}{ Konzentration [at\%] } & $\overline{\mathrm{C}}$ & 67,1 & 28,8 & 3,7 & 0,10 & 0,22 \\
& nominell & 70 & 26 & 4 & 0 & 0 \\
\hline \multirow{2}{*}{ Segregationsindex [\%] } & $\mathrm{H}$ & 1,4 & 3,1 & 8,3 & 53,2 & 36,8 \\
& $\mathrm{H}_{\text {hom }}$ & 1,6 & 3,7 & 11,9 & 73,0 & 49,5 \\
\hline
\end{tabular}

Tabelle 7.4: Quantitative Analyse von nanokristallinem $\mathrm{Fe}_{3} \mathrm{Al}-\mathrm{Cr}$ (Charge 3)

Auch die Kreuzkorrelationsanalyse (Abbildung 7.13) bestätigt diesen Trend. Für alle Elemente sind die Korrelationsparameter stets nahe bei 1, was bedeutet, dass es zu keiner Segregation bzw. Korrelation zwischen zwei Elementen kommt. Nur bei $\mathrm{P}_{\mathrm{C}, \mathrm{O}}$ sinkt der Wert etwas, so dass von einem geringfügigen gemeinsamen Auftreten von Kohlen- und Sauerstoff gesprochen werden kann.

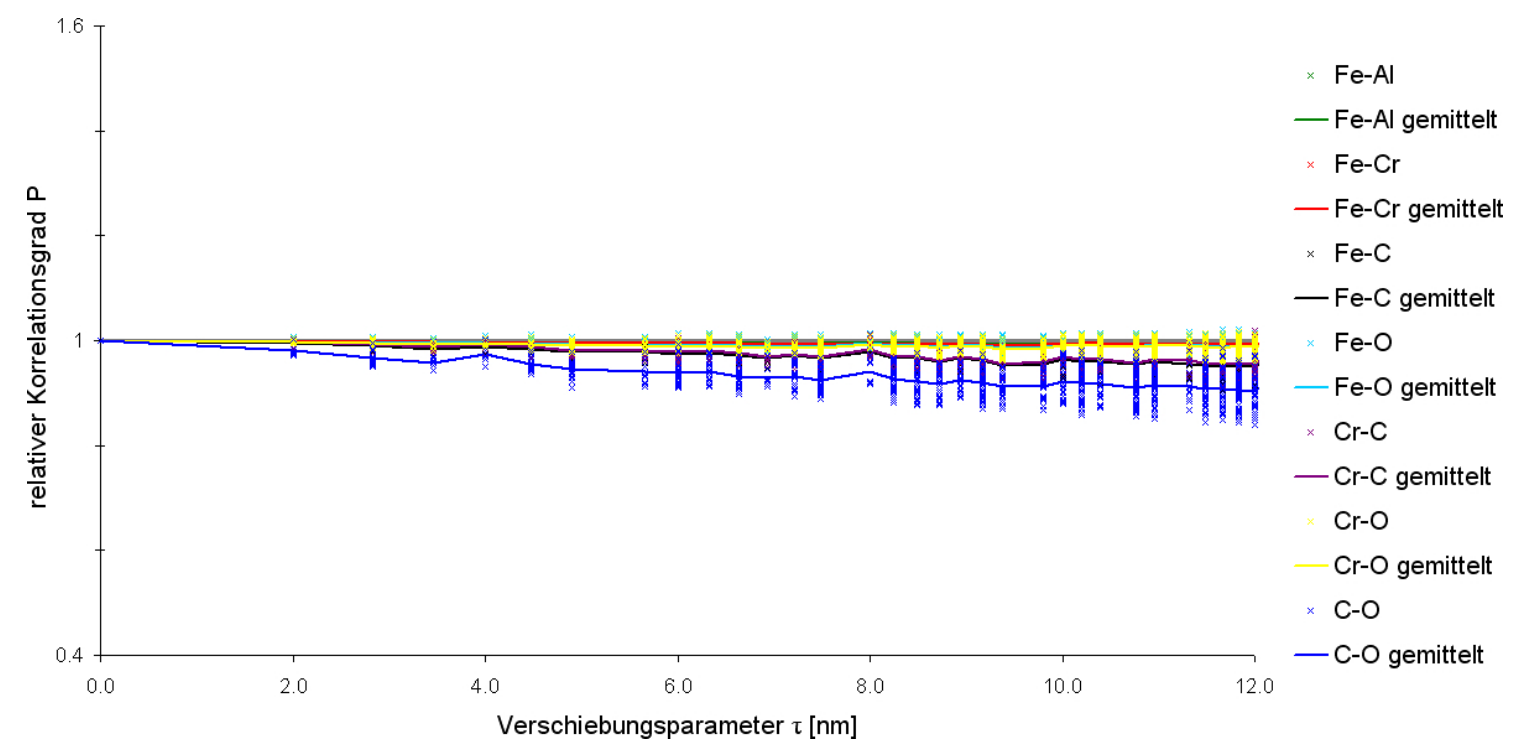

Abbildung 7.13: Kreuzkorrelationsanalyse von nanokristallinem $\mathrm{Fe}_{3} \mathrm{Al}-\mathrm{Cr}$ (Charge 3) 


\subsection{APT-Analyse an $\mathrm{Fe}_{3} \mathrm{Al}-\mathrm{Mn}$, vorlegiert}

Das vor dem Kugelmahlen eingeschmolzene $\mathrm{Fe}_{3} \mathrm{Al}-\mathrm{Mn}$ wurde als einzige Charge an einem LA-WATAP der Firma Cameca an der KAUST gemessen. Die Durchführung erfolgte bei 70K und es wurde dabei eine Spannungsanregung verwendet, aufgrund einer geringeren Detektionsrate konnte hier nur ein kleineres Messvolumen erreicht werden.

Wie die Rekonstruktionen (Abbildung 7.14) verdeutlichen, ist auch bei dieser Legierung eine sehr homogene Struktur ohne erkennbare Merkmale des nanokristallinen Gefüges zu erkennen. Eine Verunreinigung durch Kohlenstoff und Sauerstoff ist auch hier zu verzeichnen.

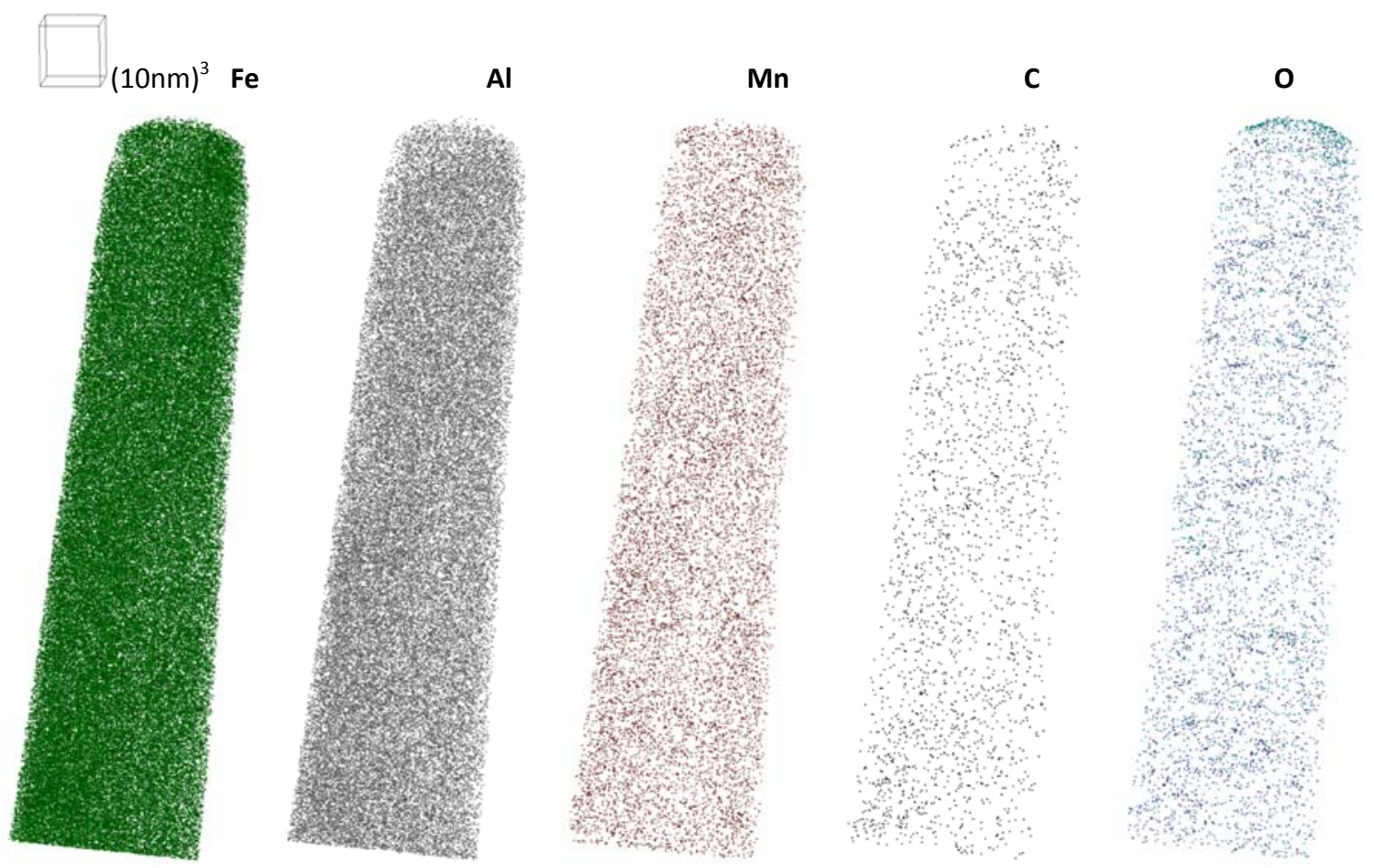

Abbildung 7.14: Rekonstruierte APT-Volumina von Fe 3 Al-Mn (Charge 4)

(Probenausschnitt von 10nm Tiefe)

Die statistischen Analysen bestätigen die gleichmäßige Verteilung der Atome. Die jeweiligen Segregationsindizes (Tabelle 7.5) entsprechen denen einer homogenen Verteilung bzw. liegen teilweise geringfügig darüber. Bei Kohlen- und Sauerstoff sind sie etwas erhöht, aufgrund ihrer geringen Konzentrationen sind diese Werte abermals nur wenig aussagekräftig.

\begin{tabular}{lcccccc}
\hline & & Fe & Al & Mn & C & O \\
\hline \multirow{2}{*}{ Konzentration [at\%] } & $\overline{\mathrm{C}}$ & 69,4 & 25,9 & 3,7 & 0,5 & 0,5 \\
& nominell & 69 & 26 & 5 & 0 & 0 \\
\hline \multirow{2}{*}{ Segregationsindex [\%] } & $\mathrm{H}$ & 1,6 & 4,3 & 14,1 & 26,8 & 39,4 \\
& $\mathrm{H}_{\text {hom }}$ & 1,6 & 4,3 & 13,3 & 23,4 & 35,2 \\
\hline
\end{tabular}

Tabelle 7.5: Quantitative Analyse an nanokristallinem Fe 3 Al-Mn (Charge 4) 
Auch die Kreuzkorrelationsanalyse (Abbildung 7.15) bestätigt das allgemein sehr einheitliche Gefüge, wenngleich die Schwankungen der Werte der relativen Korrelationsgrade deutlich stärker sind als bei anderen Analysen, da hier nur ein merklich kleineres Analysevolumen zur Verfügung steht.

Die Korrelationsgrade bleiben auch für steigendes $\tau$ nahe bei 1, im Bereich der Schwankungsbreite kann also keine Tendenz zum korrelierten Auftreten zweier Elemente gefunden werden. Der Wert $\mathrm{P}_{\mathrm{Mn}, \mathrm{C}}$ zeigt eine leicht fallende Tendenz, was einer geringfügige Neigung zum korrelierten Auftreten von Mangan und Kohlenstoff entspricht. Ähnliches kann ebenfalls für Eisen und Sauerstoff beobachtet werden.

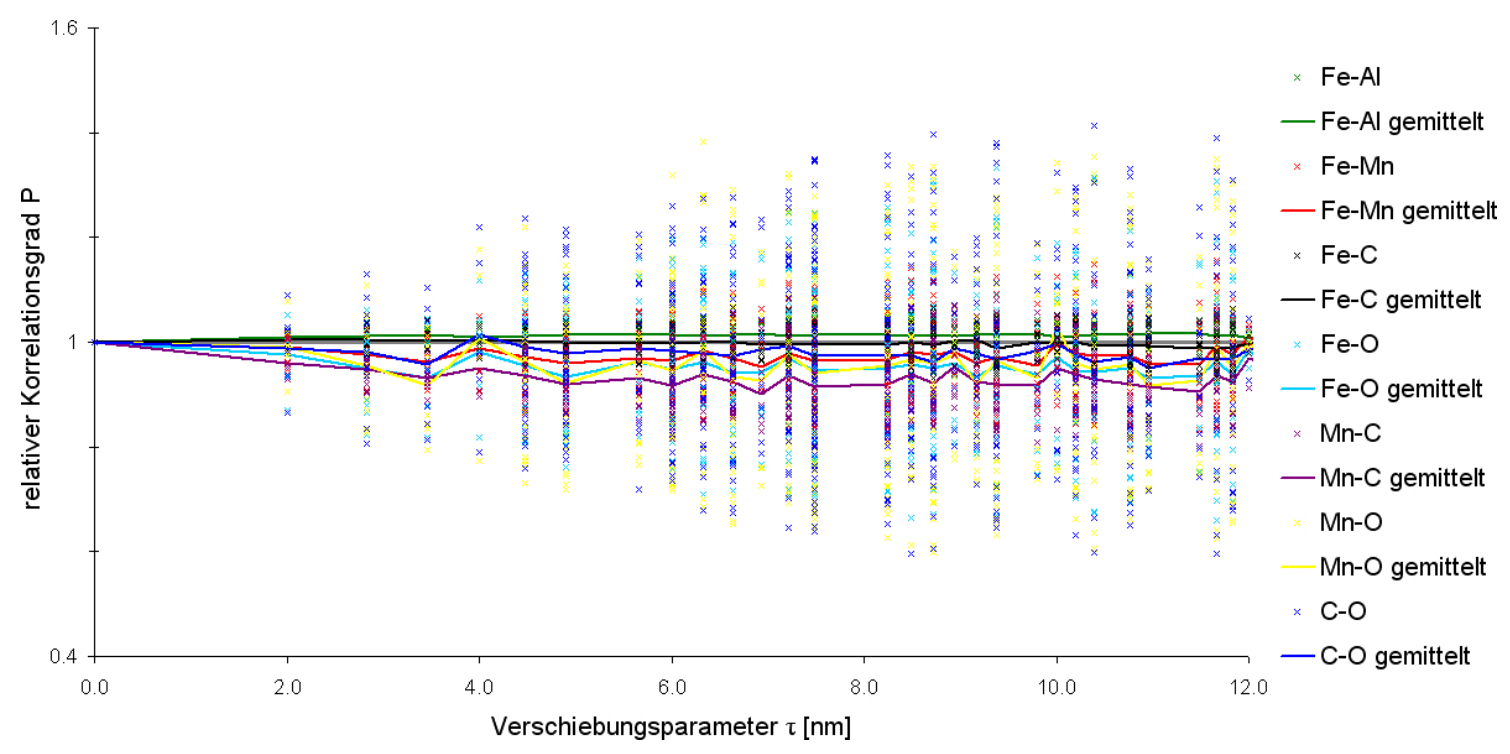

Abbildung 7.15: Kreuzkorrelationsanalyse von nanokristallinem $\mathrm{Fe}_{3} \mathrm{Al}-\mathrm{Mn}$ (Charge 4)

\subsection{APT-Analyse an $\mathrm{Fe}_{3} \mathrm{Al}-\mathrm{V}$, vorlegiert}

Die vanadiumhaltigen, nanokristallinen Proben zeigen ebenfalls eine homogene Struktur ohne erkennbare Korngrenzen. In den rekonstruierten Messvolumina (Abbildung 7.16) ist eine sehr gleichmäßige Verteilung der Legierungsbestandteile zu erkennen.

Es ist aber auch ein Artefakt zu erkennen, das von dieser Homogenität abweicht: An einer Stelle treten Kohlenstoff und Vanadium vermehrt, Eisen und Aluminium vermindert auf (rote Pfeile). Diese ausscheidungsähnliche, ca. 8nm große Erscheinung ist in dem gesamten Messvolumen nur einmal auszumachen.

Dieses Phänomen ist vergleichbar mit denen der titanhaltigen nanokristallinen Proben (7.4 und 7.8), wenngleich sie dort deutlich häufiger auftreten. Das genaue Entstehen dieses Artefakts ist nicht eindeutig zu erklären. Möglicherweise ist es eine systematische Agglomeration von Vanadium und Kohlenstoff, deren Entmischungstendenz derart geringer ist als bei $\mathrm{Fe}_{3} \mathrm{Al}-\mathrm{Ti}$, dass eine solche Anhäufung entsprechend seltener vorkommt. Aber auch das Auftreten einer relativ "frischen“ Verunreinigung durch Kohlenstoff, die noch nicht homogenisiert wurde, ist nicht auszuschließen.

Die Visualisierungen erwecken den Eindruck, dass dort deutliche Kohlenstoffagglomerationen zu finden sind. Die Gesamtkonzentration des Kohlenstoffs beträgt allerdings nur 0,23at\% (siehe Tabelle 7.6), im Bereich der Segregation ist die Kohlenstoffdichte etwa um das Zehnfache erhöht. Man kann also wieder von einem Rekonstruktionsartefakt ausgehen, genauere Diskussionen dazu folgen in 8.4. 


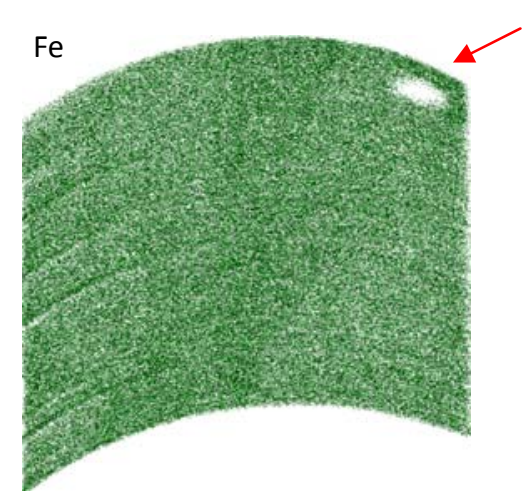

Al

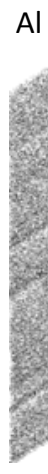

C

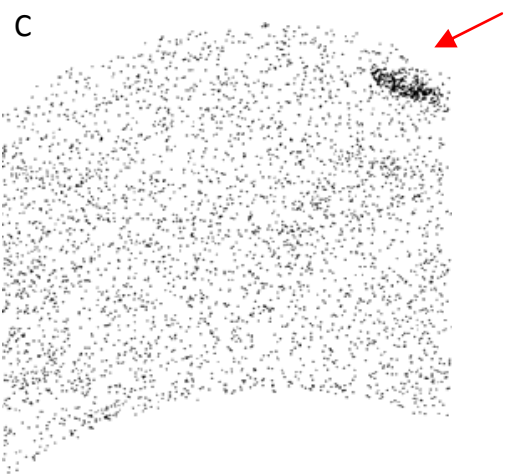

$\mathrm{O}$

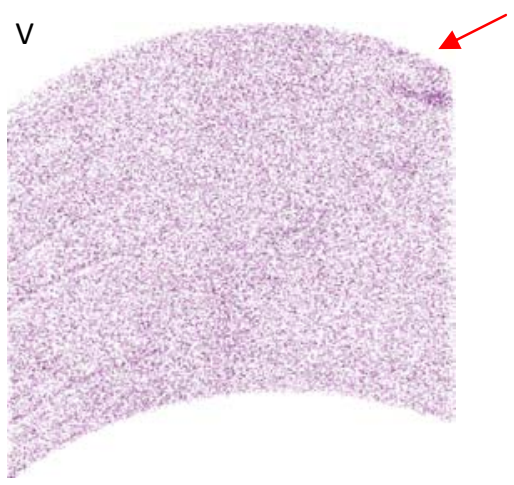

Abbildung 7.16: Rekonstruierte APT-Volumina von Fe 3 Al-V (Charge 5)

(Probenausschnitt von 10nm Tiefe, rote Pfeile: siehe Text)

Die Segregationsparameter (Tabelle 7.6) sind für alle Elemente geringer, als es einer homogenen Verteilung entspricht. Selbst für Kohlenstoff ist dies der Fall, obwohl die oben beschriebene Agglomeration markant in Erscheinung tritt. Folglich ist das einmalige Auftreten dieses Phänomens derart selten, dass es - von statistischen Gesichtspunkten - auch als natürliche Schwankung angesehen werden kann, wenngleich es intuitiv eine ausgezeichnete, sich deutlich von der restlichen Matrix unterscheidende Stelle ist.

\begin{tabular}{lcccccc}
\hline & & Fe & Al & V & C & O \\
\hline \multirow{2}{*}{ Konzentration [at\%] } & $\overline{\mathrm{C}}$ & 66,1 & 29,1 & 4,1 & 0,23 & 0,46 \\
& nominell & 70 & 26 & 4 & 0 & 0 \\
\hline \multirow{2}{*}{ Segregationsindex [\%] } & $\mathrm{H}$ & 1,2 & 2,5 & 7,5 & 34,3 & 28,4 \\
& $\mathrm{H}_{\text {hom }}$ & 1,6 & 3,6 & 11,1 & 47,9 & 33,6 \\
\hline
\end{tabular}

Tabelle 7.6: Quantitative Analyse an nanokristallinem $\mathrm{Fe}_{3} \mathrm{Al}-\mathrm{V}$ (Charge 5) 
Die Kreuzkorrelationsanalyse dieser Messung bestätigt die Homogenität, der Korrelationsparameter $\mathrm{P}$ für alle Kombinationen bleibt sehr nah bei 1, es kann also kein Segregationsverhalten erkannt werden. Nur $\mathrm{P}_{\mathrm{C}, \mathrm{O}}$ sinkt im Rahmen der Schwankungsbreite signifikant unter 1, Kohlen- und Sauerstoff zeigen also ein korreliertes, gemeinsames Auftreten.

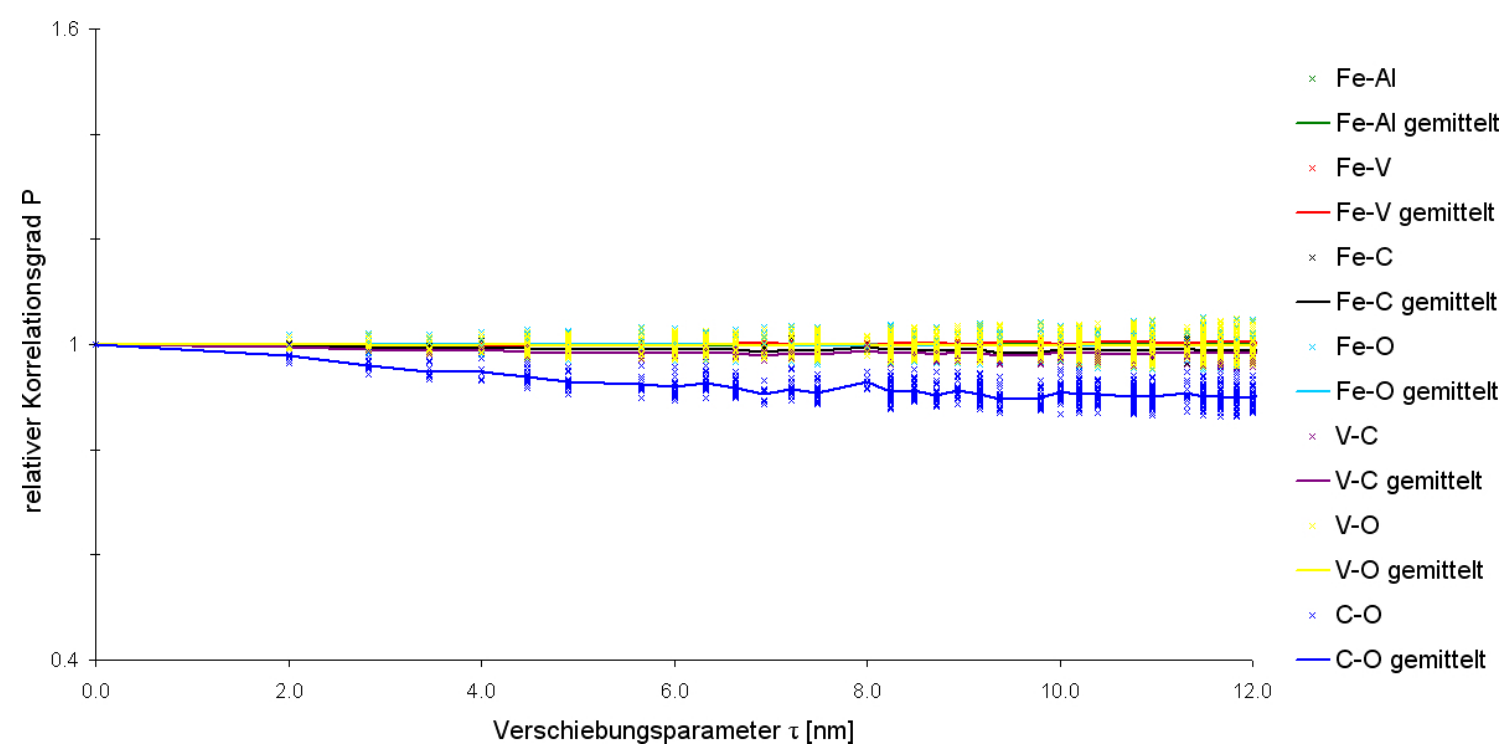

Abbildung 7.17: Kreuzkorrelationsanalyse von nanokristallinem $\mathrm{Fe}_{3} \mathrm{Al}-\mathrm{V}$ (Charge 5)

\subsection{APT-Analyse an $\mathrm{Fe}_{3} \mathrm{Al}-\mathrm{Ti}$, vorlegiert}

In der titanhaltigen und vor dem Kugelmahlen legierten Probe ist in den APT-Rekonstruktionen eine sehr inhomogene Struktur vorhanden (Abbildung 7.18). Genau wie bei den vorhergehenden Proben ist ein eindeutig nanokristallines Gefüge nicht zu erkennen. Es sind aber ausscheidungsähnliche Stellen auszumachen, an denen kaum Eisen und Aluminium, stattdessen mehr Titan, Kohlen- und Sauerstoff zu erkennen sind.

Ein zentrales Dichteprofil belegt dies quantitativ (Abbildung 7.19). In einigen Bereichen von ca. 4nm $6 \mathrm{~nm}$ Ausdehnung in z-Richtung sinken die Eisen- und Aluminiumdichte auf ca. 15\% ihres Durchschnittswertes, die Dichte der anderen Elemente steigt dort auf das Dreifache, bei Sauerstoff sogar auf das Sechsfache. Allerdings gibt es auch hier wieder den Effekt, der schon bei den mechanisch legierten titanhaltigen Proben (7.3) festgestellt wurde: In diesen Bereichen sinkt die Gesamtzahl der detektierten Atome deutlich. 

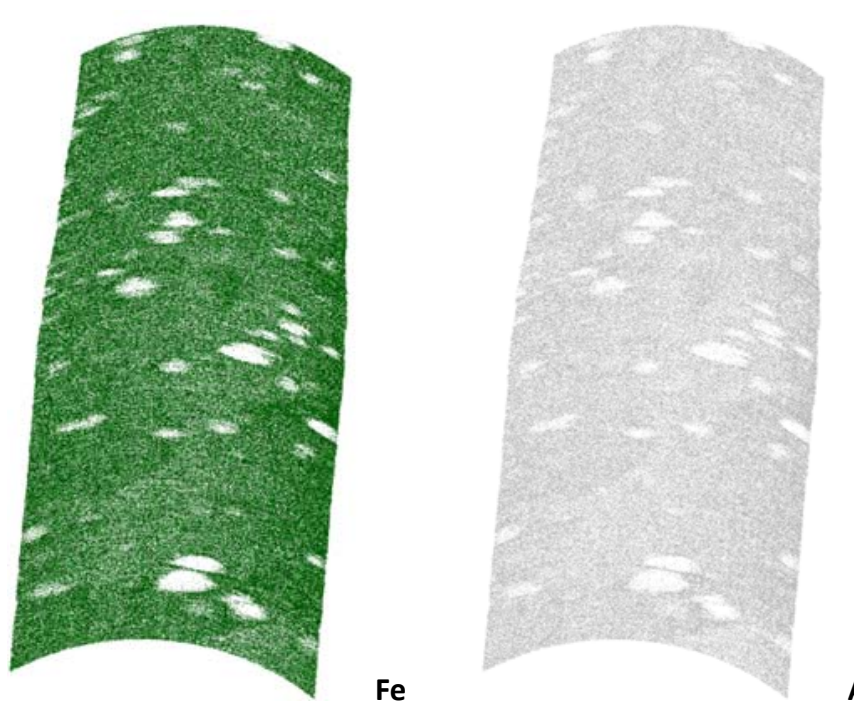

Al

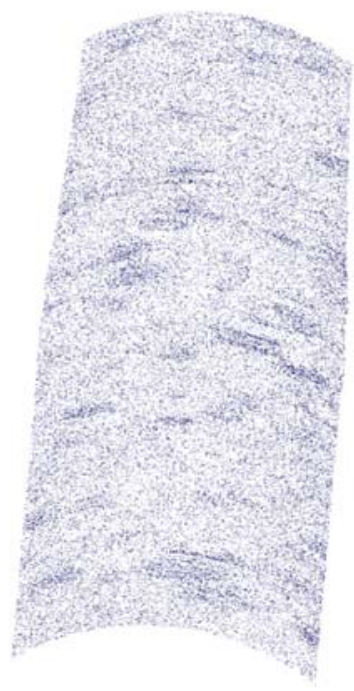

$\mathrm{Ti}$

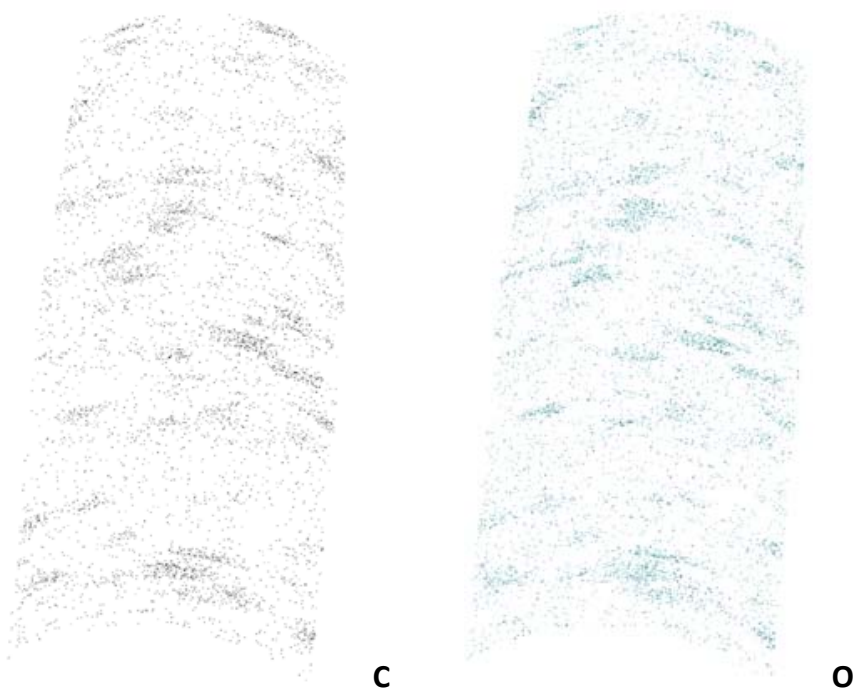

Abbildung 7.18: Rekonstruierte APT-Volumina von Fe $\mathrm{Al}-\mathrm{Ti}$ (Charge 6)

(Probenausschnitt von 10nm Tiefe)

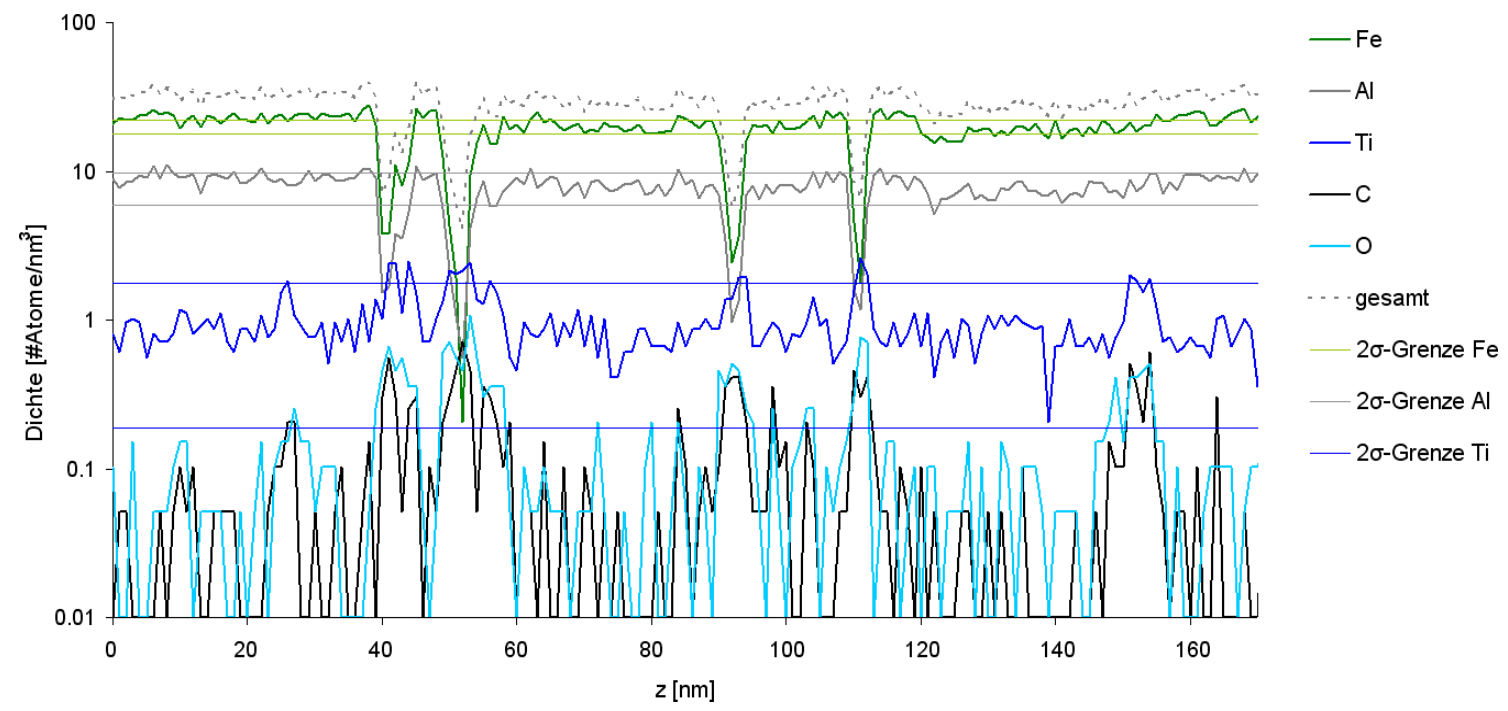

Abbildung 7.19: Eindimensionaler Dichteverlauf in nanokristallinem $\mathrm{Fe}_{3} \mathrm{Al}-\mathrm{Ti}$ (Charge 6) 
Es scheint also wiederum ein Artefakt der APT-Messung für diese immensen Dichteschwankungen verantwortlich zu sein. Dadurch werden die rekonstruierten Atompositionen derart verfälscht, dass in der visuellen Darstellung die Struktur deutlich inhomogener erscheint, als es in den Proben der Fall ist.

Das belegen die quantitativen Analysen der APT-Daten (Tabelle 7.7). Zum einen ist die Konzentration an Kohlenstoff und Sauerstoff relativ gering. Zum anderen zeigt dies der Segregationsindex $\mathrm{H}_{\mathrm{T}}$, der relativ zur homogenen Titanverteilung nicht so stark erhöht ist, wie es bei den mechanisch legierten Proben zu Verzeichnen ist (7.4).

\begin{tabular}{lcccccc}
\hline & & Fe & Al & Ti & C & O \\
\hline \multirow{2}{*}{ Konzentration [at\%] } & $\overline{\mathrm{C}}$ & 68,1 & 27,5 & 3,2 & 0,32 & 0,83 \\
& nominell & 70 & 26 & 4 & 0 & 0 \\
\hline \multirow{2}{*}{ Segregationsindex [\%] } & $\mathrm{H}$ & 1,6 & 2,8 & 17,8 & 45,4 & 33,2 \\
& $\mathrm{H}_{\text {hom }}$ & 1,7 & 2,8 & 13,9 & 45,0 & 27,7 \\
\hline
\end{tabular}

Tabelle 7.7: Quantitative Analyse an nanokristallinem $\mathrm{Fe}_{3} \mathrm{Al}-\mathrm{Ti}$ (Charge 6)

Folglich wird die Struktur in Abbildung 7.18 bzw. Abbildung 7.19 aufgrund der Darstellungseffekte als zu inhomogen illustriert. Eine direkte Möglichkeit, diese Rekonstruktionsartefakte zu vermeiden, gibt es nicht. Es handelt sich vermutlich um einen Effekt, der vergleichbar mit dem Effekt der verspäteten Evaporation ist, jedoch im größeren Maßstab (siehe Kapitel 4 und Kapitel 5). Dadurch kommt es - genau wie bei den Kristallebenen - zu einer Verschiebung in der Rekonstruktion. Genauer wird auf diesen Effekt in 8.4.2 eingegangen.

Eine Möglichkeit, diese Erscheinung indirekt zu umgehen, ist die einer nachträglichen Verschiebung der Atome, z.B. derart, dass als Ergebnis das rekonstruierte Material eine konstante Dichte aufweist [101, 102]. Diese Methodik ist dahingehend ungenau, dass die Annahme konstanter Dichte nicht gegeben sein muss und z.B. durch interstitiell gelöste Fremdatome deutlich vom wahren Wert abweichen kann. Des Weiteren ist nicht vorauszusagen, welche Elemente verschoben werden müssten. Lag eine gemischte Phase vor, bei der Z.B. Eisen und Aluminium der verfrühten Evaporation unterlagen, müssten auch nur diese verschoben werden. Waren Titan, Kohlenstoff und Sauerstoff maximal segregiert, müsste der gesamte Bereich um die Agglomeration angepasst werden. Für diesen Fall ließe sich abschätzen, dass die titanreichen Gebiete in der Rekonstruktion etwa drei- bis viermal zu groß dargestellt werden würden. Damit läge ihre wahre Größe im Bereich von $1 \mathrm{~nm}-2 \mathrm{~nm}$. Allerdings ist von einer vollkommenen Segregation aufgrund der Dichteverläufe und dem Vorhandensein von Fe und Al nicht auszugehen. Die Agglomerate sind also vermutlich größer (sieh auch 8.5.4).

Das beobachtete Verhalten von gemeinsamen Ti-, C- und O-Agglomerationen kann durch die Kreuzkorrelationsanalyse bestätigt werden, die Parameter $\mathrm{P}_{\mathrm{Ti}, \mathrm{C}}, \mathrm{P}_{\mathrm{Ti}, \mathrm{O}}$ und $\mathrm{P}_{\mathrm{C}, \mathrm{O}}$ sinken deutlich für $\tau>0$. Das gleichlaufende Absinken der Eisen- und Aluminiumkonzentration an diesen Stellen zeigt sich ebenfalls durch ein Absinken des Parameters $\mathrm{P}_{\mathrm{Fe}, \mathrm{Al}}$. Dieses ist zwar wegen der jeweils hohen Konzentration nur gering ausgeprägt, im Rahmen der Streuung ist es aber signifikant.

Anhand der weiteren Parameter, die für $\tau>0$ ansteigen, wird deutlich, dass Eisen und Aluminium eine ausgeprägte Segregation zu den übrigen Elementen zeigen. 


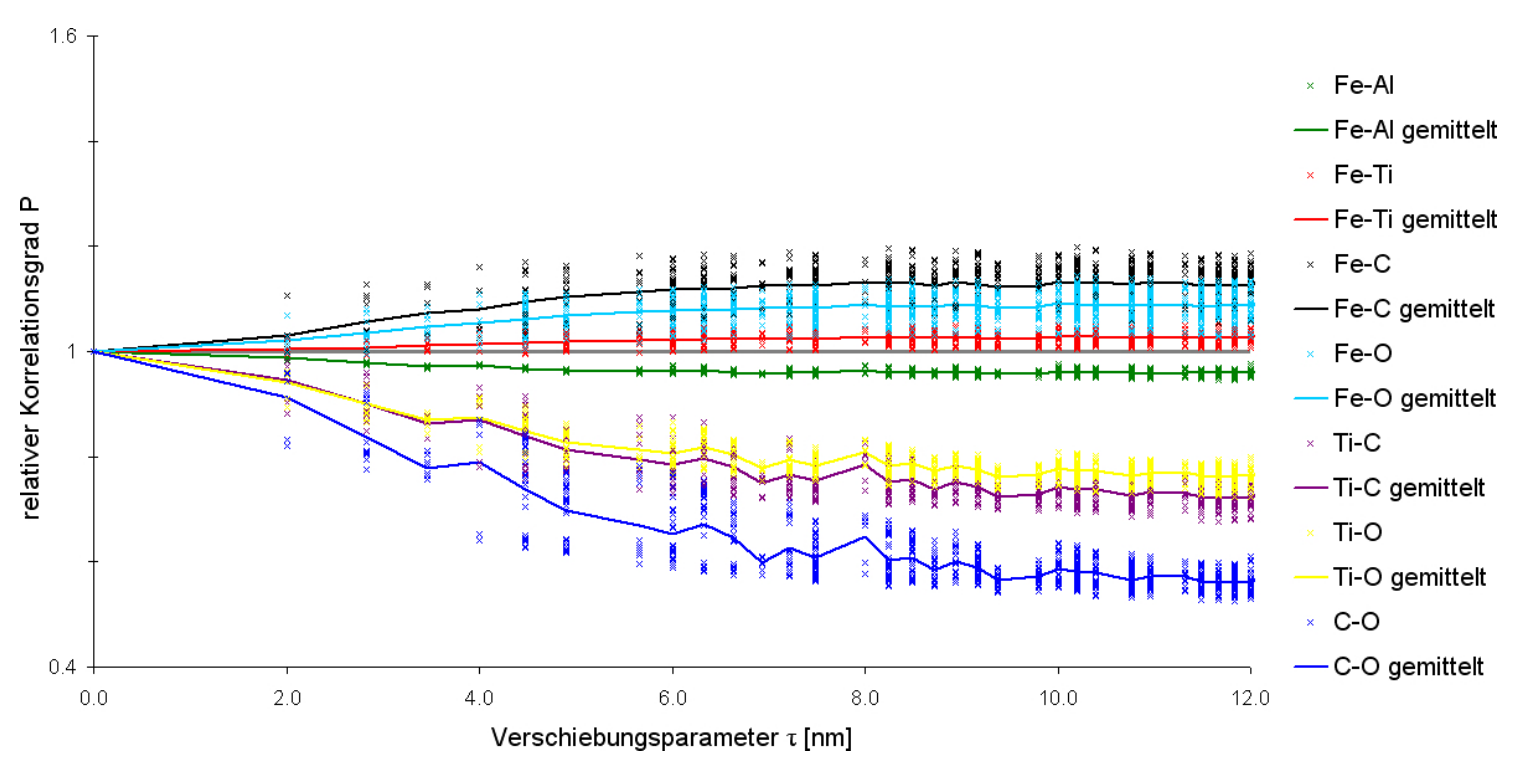

Abbildung 7.20: Kreuzkorrelationsanalyse von nanokristallinem $\mathrm{Fe}_{3} \mathrm{Al}-\mathrm{Ti}$ (Charge 6)

Für diese Probe wird also deutlich, dass Titan zusammen mit Kohlen- und Sauerstoff agglomeriert vorliegt. Aufgrund der Schwierigkeiten und Ungenauigkeiten bei der APT-Rekonstruktion ist nicht eindeutig zu benennen, ob es sich eher um die Bildung einer zweiten Phase oder um Schwankungen der Konzentration z.B. an Korngrenzen handelt. Letzteres erscheint u. a. aufgrund der Vorzugsrichtung der Agglomerationen als unwahrscheinlicher. Da sie parallel zur Partikeloberfläche orientiert sind (der Probenblock wurde vertikal aus einem Partikel herausgeschnitten), sind sie allem Anschein nach herstellungsbedingt. Mögliche Erklärungen für diese Phänomenologie, die auch in 7.3 beobachtet wurde, werden in 8.5 diskutiert. 


\section{Kapitel 8: Diskussion und Schlussfolgerungen}

Nun sollen die Analysen der vorigen Kapitel nochmals in ihrer Gesamtheit reflektiert werden. Dazu werden die verschiedenen Messergebnisse unterschiedlicher Analysen zusammengefasst und verglichen, um allgemeine Aussagen zu schlussfolgern. Außerdem werden die Zusammenhänge und die Aussagekraft der Ergebnisse diskutiert und im gesamtwissenschaftlichen Kontext betrachtet.

\subsection{Qualitative Analyse von APT-Messungen an massiven, geordneten Proben}

In dieser Arbeit wurden erstmals für ternäre Eisenaluminide mit der Atomsondentechnologie systematisch für die drei kristallographischen Hauptrichtungen erfolgreich Messungen angefertigt. Davon sollen zunächst allgemeine Messeigenschaften betrachtet werden.

\subsubsection{AtomVicinity-Dichteprofile}

Die AtomVicinity-Analyse ist ein sinnvolles Mittel, die direkte Umgebung eines Atoms mit höchster Signifikanz zu bestimmen. Insbesondere ist hier nochmals zu erwähnen, dass globale Fehlrekonstruktionen (z.B. verzerrte Rekonstruktion) ausgeglichen werden und nur die lokalen (also die genannten Peakverschiebungen) erkennbar werden. Dadurch können die durch APT ermittelten Ebenenabfolgen mit höchster Auflösung sichtbar gemacht werden. Gerade für die Elemente mit geringer Konzentration, die in einem einfachen Dichteprofil kaum von einem Rauschsignal unterschieden werden können, sind dadurch erst die genauen Ebenenpositionen zu ermitteln.

In AtomVicinity-Dichteprofilen sind die mittigen Peaks des Aluminiums jeweils höher als die danebenliegenden Al-Peaks. Dies ist mit der Berechnungsweise der Atomumgebung zu erklären und liegt an zwei Effekten:

- Das zentrale Atom, dessen Umgebung betrachtet wird, hat eine Antreffwahrscheinlichkeit von 100\%. Bei den dazugehörigen Umgebungsatomen ist diese jedoch nur so groß wie die Detektionswahrscheinlichkeit der APT, also ca. 50\%.

- Außerdem wird das zentrale Atom genau an die Position $(0,0)$ platziert, während die anderen Atome in ihrer relativen Positionierung die Messungenauigkeit der APT übernehmen. Dadurch wird der mittlere Peak zwangsläufig schmaler als die anderen.

Die wahre Messungenauigkeit der APT ist also an dem mittleren Peak nicht auszumachen, denn er besteht aus Atomen, die per Definition genau an der mittigen Position sitzen und aus Atomen der gleichen Ebene, die relativ dazu mit der wahren Messungenauigkeit positioniert sind.

Die Tatsache, dass sich auch für die anderen Elemente die mittleren Peaks von den anderen unterscheiden, ist ein Hinweis auf bestimmte Evaporationseffekte und wird in 8.2.2 diskutiert. 


\subsubsection{Evaporationsverhalten von $\mathrm{Fe}_{3} \mathrm{Al}$}

In den AtomVicinity-Dichteprofilen zeigt Eisen eine Verschiebung nach links relativ zur erwarteten, theoretischen Position, insbesondere in den $<100\rangle$ - und <111>-Richtungen. Das bedeutet, dass es der verfrühten Evaporation unterliegt und folglich mit einer zu kleinen z-Komponente rekonstruiert wird, in den Dichteprofilen also weiter links liegt (siehe 4.2).

Dieser Effekt bedeutet, dass Eisen eine kleinere Feldverdampfungsfeldstärke hat als das Aluminium. Die FIM-Bilder, bei denen gezeigt wurde, dass Aluminium das abbildende Element ist und damit die größere Evaporationsfeldstärke besitzt, stützen diese Betrachtung (siehe 4.1 und [77]).

Auch die Berechnungen der Elementkonzentrationen anhand der APT-Daten bestätigen dies: Hier wurde für Aluminium eine zu hohe Konzentration gemessen. Dies ist eine Folge davon, dass Aluminium mit seiner höheren Evaporationsfeldstärke den Verdampfungsprozess kontrolliert. Einige Eisenatome verdampfen infolgedessen unmittelbar nach einem Aluminiumatom, weil ihre Verdampfungsfeldstärke bereits erreicht war und sie nur durch das Aluminium zurückgehalten wurden (korrelierte Evaporation). Diese Fe-Atome werden dann aber technisch bedingt mit geringerer Wahrscheinlichkeit detektiert (siehe 4.5). Dass dieser Effekt das gesamte Evaporationsverhalten während der APT-Messung beeinflusst, zeigen die Rohdaten des Detektors: bis zu 9\% aller gemessenen Eisenatome kommen in dem gleichen Spannungspuls an etwa gleicher Stelle an dem Detektor nach einem Aluminiumatom an. Wie viele Eisenatome das gleiche Verhalten zeigen, jedoch nicht detektiert werden, kann daraus nicht ermittelt werden. Ihre Zahl müsste noch einmal so groß sein, um die nominelle Konzentration zu erreichen.

Folglich besitzt die Konzentrationsbestimmungen mit APT eine gewisse systematische Ungenauigkeit. Daher sind für diese Studien die Ergebnisse der EDX-Analysen deutlich präziser und aussagekräftiger. Sie bestätigen die nominellen Konzentrationen, auch für alle Zusätze.

Ebenfalls wurde auch gezeigt, dass dieser Effekt deutlich richtungsabhängig ist, was durch die unterschiedlichen Ebenenabfolgen erklärt werden kann (4.5). Eine solche Richtungsabhängigkeit ist aber auch bei der Peakverschiebung in den AtomVicinity-Dichteprofilen zu erkennen (4.2). Die $<110>-$ Orientierung weist jeweils nur eine sehr geringe Peakverlagerung auf, auch bei allen ternären Legierungen. Der Grund dafür ist der größte Ebenenabstand in dieser Richtung, was eine verfrühte Evaporation aus darunter liegenden Ebenen wiederum unwahrscheinlicher macht.

Diese Rekonstruktionseffekte, die sich in der Peakverschiebung widerspiegeln, stellen auch eine Herabsetzung der Messgenauigkeit dar. Denn die Fehlpositionierung von Eisen relativ zu Aluminium um bis zu eine Kristallebene bedeutet eine zusätzliche Messungenauigkeit von in diesem Fall bis zu $2 \AA$. Da die Messgenauigkeit von Atomsonden im Allgemeinen mit $2 \AA$ - $4 \AA$ angegeben wird [46, 48], kann man allgemein ableiten, dass in einer Legierung, in der eine verfrühte Evaporation eines Elementes eine relevante Rolle spielt, dieser Effekt wesentlich zum Absinken der Auflösung beiträgt. Dieses Absinken ist jedoch auch wieder richtungsabhängig und kann dann sogar innerhalb einer Legierung sowohl stark als auch sehr gering ausgeprägt sein, wie es hier das Beispiel $\mathrm{Fe}_{3} \mathrm{Al}$ für die <111>- bzw. $<110>$-Richtungen zeigt. 


\subsection{Simulation von APT-Messungen}

\subsubsection{Allgemeine Betrachtung und Weiterentwicklungen}

Die Simulationen von APT-Messungen zeigen insgesamt eine hohe Übereinstimmung zu den entsprechenden Messungen, die jeweiligen Dichteprofile weisen im Wesentlichen eine gute Deckungsgleichheit auf. Insbesondere soll hier betont werden, dass die stark richtungsabhängige Peakverschiebung der Messungen ebenfalls in den Simulationen zu erkennen ist. Diese Tatsache stellt einen relevanten Aspekt dar und ist daher ein wichtiger Fortschritt.

Dafür hat sich gerade die Verwendung des EAM-Modells zur Berechnung der Bindungsenergien als eine sinnvolle Erweiterung des Simulationsalgorithmus erwiesen. So haben anfängliche Versuche, die Bindungsenergie nur durch Paarpotentiale zu bestimmen, nicht zu akzeptablen Ergebnissen geführt. Z.B. konnte die unterschiedlich starke Peakverschiebung von Eisen in $<100>$ - und <110>-oreintierten Dichteprofilen der binären Legierung nicht simuliert werden. Ausserdem war es auch nicht möglich, die sehr deutliche Verlagerung der Chrom-Peaks durch realistische, reine Paarpotentiale zu simulieren.

Auch andere physikalischen Erkenntnisse verdeutlichen die Ungenauigkeit von Paarpotentialen, z.B. können Leerstellenenergien und Bindungsverhalten an Oberflächen nur ungenügend berechnet werden. Gerade dafür hat sich das EAM-Modell etabliert, da es die Prinzipien von atomaren Wechselwirkungen und DFT (Bindungsenergie als Elektronendichtefunktional) beinhaltet. Es wird dabei an einfach zu ermittelnden entsprechenden Konstanten wie NN-Abstand, Atomvolumen, Sublimations- und Leerstellenenergie geeicht. Die verwendete Einbettungsfunktion - die Wurzelfunktion - repräsentiert dabei als ein einfaches Modell den Einfluss der Zustandsdichte [103], sie bietet aber für eine Anwendung im Rahmen dieser Arbeit eine hinreichende Genauigkeit [104].

\subsubsection{Mess- und Simulationsgenauigkeit}

Beim Vergleich der simulierten und gemessenen Dichteprofile ist zu erkennen, dass die Position der Peaks gute Übereinstimmung aufweist und Abweichungen sich im Bereich von unter 0,6̊̊ bewegen.

Zur Einschätzung dieser Abweichungen muss zum einen die allgemeine Messgenauigkeit der APT beachtet werden. Nominell liegt diese bei ca. $2 \AA$ in Messrichtung [46, 47]. Allerdings kann anhand der Dichteprofile erkannt werden, dass sie für den vorliegenden Messungen höher ist, betrachtet man die

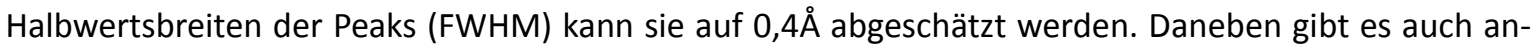
dere systematische Einflüsse auf die relativen Ebenenabstände wie beispielsweise unterschiedliche Messbedingung. So können unterschiedliche Messspannungen zu verschiedenen Krümmungsradien $r_{k}$ führen. Wie in 4.6 beschrieben können dadurch - auch wenn das Verhältnis $r_{s} / r_{k}$ konstant bleibt - die relativen Peakpositionen geringfügig um bis zu 0,2Å verschoben sein. Auch die Wahl des Analysevolumens für die AtomVicinity-Dichteprofile beeinflusst diese unmittelbar. Die Ebenenauflösung in APTDaten ist am höchsten in direkter Nähe zum Polmittelpunkt. Daher muss dort dieses Volumen positioniert sein. Ein zu klein gewähltes Volumen senkt die statistische Genauigkeit, wird es zu groß gewählt, werden auch Bereiche mit schlechterer Auflösung miteinbezogen. Dies macht sich allerdings nicht unbedingt nur in einem höheren Rauschsignal bemerkbar, sondern z.B. auch in einer seitlichen Flanke eines Peaks, wodurch sich dessen mittlere Position ändert.

Insgesamt kann man also feststellen, dass die Genauigkeit, mit der die Messungen simuliert werden können, innerhalb des Bereichs der Messgenauigkeit und -schwankungen liegt. Somit können diese als zufriedenstellend angesehen werden. 
Auffälligere Abweichungen gibt es hingegen durchaus in den genauen Peakformen. Diese zeigen teilweise Besonderheiten, die u. a. auch Hinweise auf bestimmte Evaporationseffekte geben können. Sie sind gerade bei Eisen und den ternären Zusätzen in den AtomVicinity-Dichteprofilen zu erkennen.

Abweichende Peakformen des mittleren Peaks treten z. B. bei Eisen auf, sie sind im Allgemeinen schmaler als die anderen Peaks. Dies ist jedoch zunächst erstaunlich, da Eisen kein Zentralatom an $(0,0)$ besitzt, d.h. die Begründung für abweichende mittlere Peaks des Aluminiums (8.1.1) ist nicht gültig. Stattdessen wird für alle Eisenatome nur die relative Lage zum Zentralatom bestimmt, damit müssten aber dessen Peakformen alle sehr ähnlich sein, da alle der gleichen Messungenauigkeit unterliegen.

Der Grund für dieses Verhalten ist wiederum ein Rekonstruktionseffekt, der durch die korrelierte Evaporation von Aluminium und Eisen entsteht. Dadurch wird, wie in 4.2 beschrieben, das Evaporationsverhalten des Eisens durch das Aluminium determiniert. Folglich gibt es dann für den mittleren Peak doch eine Beeinflussung durch das Zentralatom in den Dichteprofilen, wodurch der mittlere Peak des Eisens nicht nur der allgemeinen Messungenauigkeit unterliegt. In den Darstellungen ist das dann der Fall, wenn der Peak eines anderen Elementes nach links verlagert ist und sich bis an das Aluminium verschiebt (z.B. Fe und $\mathrm{Cr}$ in <111>-Richtung, Abbildung 4.6 und Abbildung 5.1). Auch andere Effekte, die in den Simulationen nicht beachtet werden, können die Peakform beeinflussen, wodurch diese auch nicht immer mit hoher Übereinstimmung simuliert werden kann. Denkbar für solche Effekte ist z.B. ein gemeinsames Evaporieren mehrerer Atome. Es ist bekannt, dass in APT-Messungen ganze Moleküle verdampfen können (z.B. Polymeerteile [105], Oxide [106]). Für die vorliegenden Legierungen könnte z.B. ein gemeinsames Ablösen von z.B. Fe-Al-Verbindungen vermutet werden, welche erst nach dem Ablösen zerfallen. Ein solcher Effekt kann die Peakform prinzipiell beeinflussen: Dadurch kann beispielsweise eine seitliche Flanke entstehen, wie es bei Vanadium in <111>-Richtung (Abbildung 5.8) beobachtet werden kann. Er kann aber auch von der allgemeinen Messungenauigkeit überlagert werden und nur bei den mittleren Peaks auftreten, wie z.B. bei Chrom in <100>-Richtung (Abbildung 5.1).

Den eben dargestellten Effekt kann man auch noch allgemeiner beschreiben: In der Simulation wird nach jedem Evaporationsschritt das Bindungsverhalten neu berechnet. Dabei wird davon ausgegangen, dass sich die Probe wieder im Gleichgewichtszustand befindet. Dies muss aber in der Realität nicht der Fall sein: Ein eben evaporiertes Atom hinterlässt seine direkte Umgebung eine Zeitlang in einem NichtGleichgewichtszustand. Dieser würde dafür sorgen, dass die Evaporationswahrscheinlichkeit eines Nachbaratoms für eine nicht verschwindende Zeit verändert ist, so dass das Evaporationsverhalten einiger Atome insgesamt geändert sein kann. Dieses Phänomen könnte dann z.B. das korrelierte Verdampfen begünstigen, ein gemeinsames Ablösen zweier Atome als Molekül wäre dann der Extremfall.

Solche Einflüsse auf die Peakform bzw. auf das Evaporationsverhalten sind quantitativ nur schwer zu fassen. Daher wurde im Rahmen dieser Arbeit darauf verzichtet, die Simulationen dahingehend zu optimieren. Stattdessen wurde - wie in 8.2.2 bereits beschrieben - die Peakform nicht als wesentlicher Vergleichsmaßstab angesetzt. Bei den Legierungen, bei denen solche Effekte sehr deutlich auftreten (z.B. $\mathrm{Fe}_{3} \mathrm{Al}-\mathrm{V}$ ), sorgen sie bei der Abschätzung der Ordnungsgrade erhöhte Ungenauigkeiten.

\subsubsection{Paarpotentiale}

An dieser Stelle soll abgeschätzt werden, wie genau Paarpotentiale durch den Vergleich vom Simulation und Messung bestimmt werden können. Für die Minimumposition ist bei elementreinen Potentia-

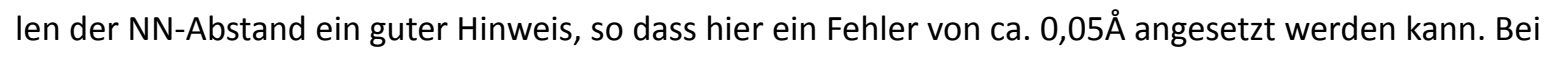
gemischten Potentialen gibt es diese „natürliche“ Vorgabe nicht. Hier können die Ungenauigkeiten im Wesentlichen über ihren Effekt bei den Simulationen abgeschätzt werden, so dass ein Fehler von $0,15 \AA$ einkalkuliert werden muss. Allerdings kann eine Veränderung von $0,2 \AA$ wie in der Betrachtung zu 
$\mathrm{Fe}_{3} \mathrm{Al}-\mathrm{Ti}$ (5.4.2) derart andere Ergebnisse hervorrufen, dass die Minimumsposition teilweise genauer eingegrenzt werden kann.

Ein Hinweis für die Genauigkeit der Potentialtiefe - die im Allgemeinen entscheidendere Angabe -bietet die Bandbreite der Literaturangaben. Als Beispiel soll das Eisenpotential dienen, zu dem es die meisten Angaben gibt. Deren Werte liegen zwischen $0,19 \mathrm{eV}$ und $0,39 \mathrm{eV}$, der Großteil davon bei ca. 0,3eV [72, 80-87]. Zum Vergleich sind diese Fe-Fe-Potentiale in Abbildung 8.1 dargestellt. Man erkennt, dass es teilweise deutliche relative Abweichungen unter den Potentialen gibt. Folglich kann ein systematischer Fehlerwert von etwa 0,05eV angenom-

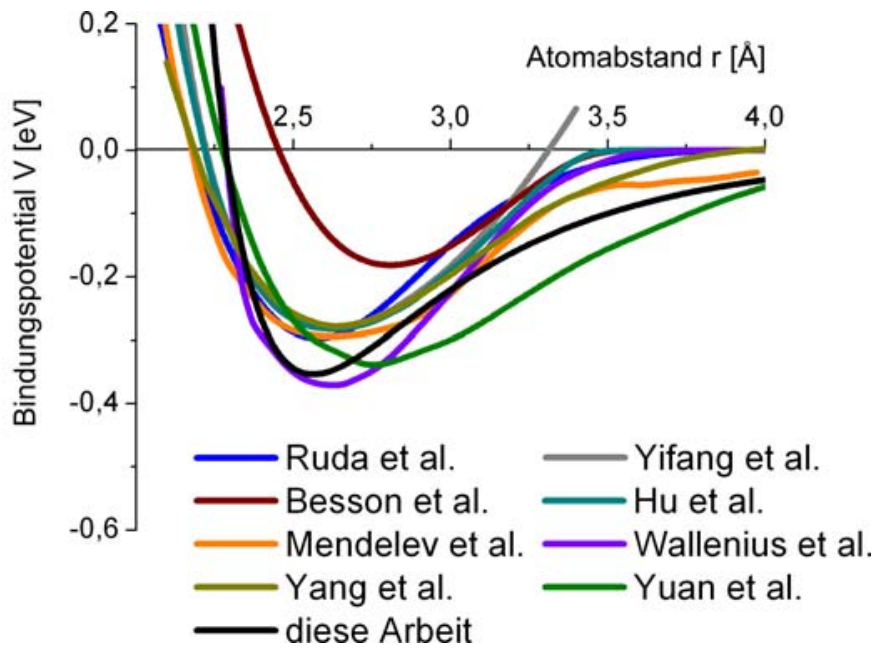

Abbildung 8.1: Fe-Fe-Paarpotential verschiedener Studien men werden. Bei Potentialen mit weniger oder keinen Angaben in der Literatur kann auch ein größerer Fehler von bis zu 0,1eV bei den hier ermittelten Potentialen nicht ausgeschlossen werden.

Des Weiteren ist zu beachten, dass im Allgemeinen Paarpotentiale für eine Temperatur von OK berechnet bzw. an auf OK extrapolierten Daten geeicht werden. Die APT-Messungen wurden alle bei 30K, die der titanhaltigen Legierung bei 70K durchgeführt. Gerade der Vergleich zwischen diesen Messungen macht deutlich, dass die Messtemperatur ebenfalls einen Einfluss auf das Dichteprofil hat. Bei $\mathrm{Fe}_{3} \mathrm{Al}-\mathrm{Ti}$ hat es den Anschein, dass die verfrühte Evaporation noch stärker ausgeprägt ist. Das kann an veränderten Bindungspotentialen liegen, ebenso ist ein Einfluss auf das lonisationsverhalten nicht auszuschlieBen, was dann durch den Faktor $\mathrm{K}$ repräsentiert wird. Auch diese Temperaturabhängigkeit der APTMessung bedeutet, dass sich die verwendeten Paarpotentiale - die dann auf 30K bzw. 70K angepasst sind - nur mit einer gewissen Abweichung mit Literaturwerten vergleichen lassen.

Beide Aspekte - die allgemeine Genauigkeit von Paarpotentialen und die Temperaturabhängigkeit führen dazu, dass ein Fehlerwert von bis zu 0,1eV einkalkuliert werden muss.

\subsubsection{Simulationsparameter}

Die Simulationsparameter $\mathrm{K}$ und $\mathrm{D}$ wurden an den Messungen der binären Legierung geeicht. Ihre Genauigkeit kann jeweils mit etwa $\pm 20 \%$ angegeben werden. Dieser Wert stellt aber keinen direkte Fehlerangabe dar, sondern beschreibt, in welchem Rahmen Simulationen mit annähernd gleich guter Übereinstimmung durchgeführt werden können. Auch bei höheren Abweichungen können noch prinzipielle Merkmale der Messungen simuliert werden. Das bedeutet, dass diese Parameter keinen sehr großen, substanziellen Einfluss auf das allgemeine simulierte Evaporationsverhalten in dem Sinne haben, dass eine kleine relative Veränderung bereits ein deutlich anderes Simulationsergebnis hervorruft. Diese Tatsache wird vom Autor als ein positiver Aspekt des Simulationsalgorithmus gewertet, weil sie bedeutet, dass die simulierten Ergebnisse im Wesentlichen durch die Materialparameter und der Berechnung von $E_{B}$ geprägt werden.

An dieser Stelle soll aber noch einmal die Bedeutung der Parameter K und D diskutiert werden. Letzterer kann quantitativ kaum überprüft werden. Anders bei K, bei dem der Wert von 0,35 bedeutet, dass die lonisierungsenergie nicht vollkommen mit einkalkuliert wird. Dies ist zunächst ein Unterschied zum Charge-Exchange- oder dem Image-Hump-Modell (siehe 3.3.2), bei dem diese Energie komplett ein- 
geht. Allerdings weisen auch diese Modelle Ungenauigkeiten in der Bestimmung des Evaporationsverhaltens auf [48].

Die Verwendung und Herleitung eines Faktors $\mathrm{K}$ wurde in 3.3.2 erläutert. Er ergibt sich als lineare Approximation des Einflusses der lonisierungsenergie, wenn man den Verlauf des Potentials nicht vernachlässigt und von realistischen Randbedingungen ausgeht (entsprechend Abbildung 3.7).

Die Tatsache, dass $K=0,35$ verwendet wurde, kann so interpretiert werden, dass die lonisierung zum Zeitpunkt der Überwindung des Gesamtpotentialmaximums nicht abgeschlossen ist. Sie spiegelt wider, dass ein instantanes lonisieren nicht realistisch ist und stattdessen auch für diesen Teilprozess ein Verlauf angenommen werden muss. Dies würde dann bedeuten, dass die tatsächlichen Energiewerte verringert sind. Auch FORBES beschrieb den Evaporationsablauf als einen Prozess, bei dem sich Ablösung, Ionisierung und Beschleunigung des Atoms überlagern (zeitlich und örtlich) [67]. Daher schilderte er die lonisierung als einen kontinuierlichen Prozess und bezeichnete dies als charge drain, Fließen der Ladung, das quantitativ durch einen Koeffizienten (Ionisierungsgrad) beschrieben werden kann. Diese Betrachtung entspricht dem Vorfaktor K.

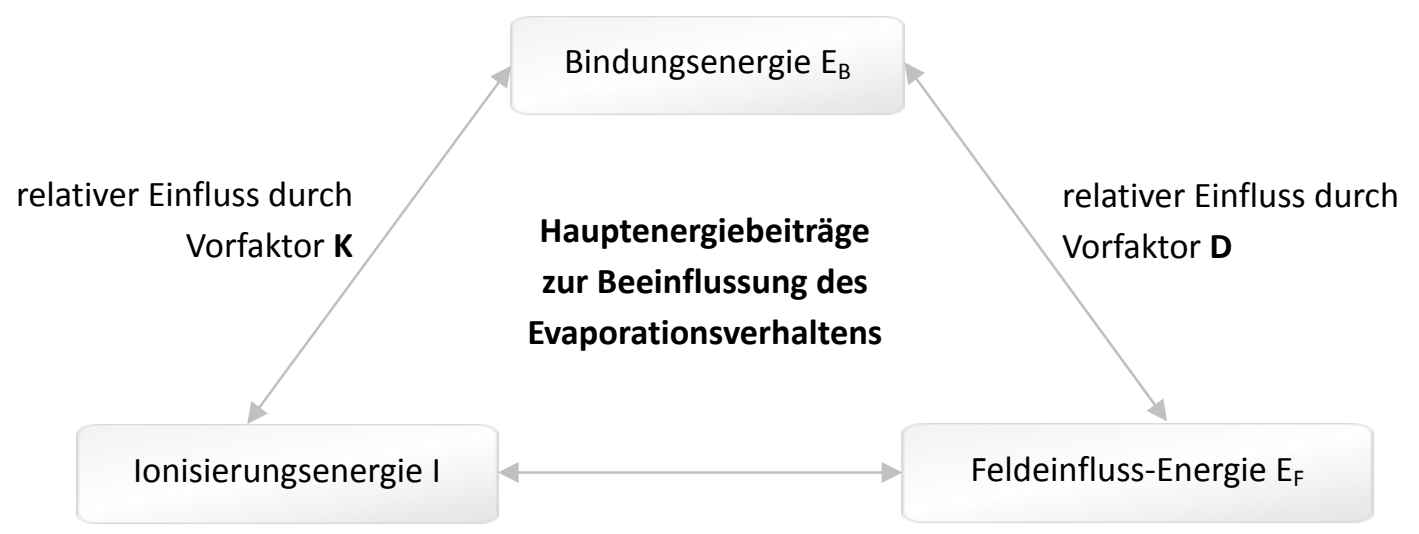

Abbildung 8.2: Schematische Beziehung der Energiebeiträge der Feldevaporation

Man kann diese Betrachtung aber noch allgemeiner fassen: Unabhängig von dem genauen Verlauf der Evaporation wird dieser Ablauf im Wesentlichen durch drei Energiebeiträge bestimmt: Durch die Bindungsenergie $E_{B}$, die lonisationsenergie I und die Energiekomponente $E_{F}$, die durch das Feld verursacht wird. Geht man davon aus, dass diese Energiebeiträge jeweils berechnet werden können, so muss für eine realistische Simulation jeweils der relative Einfluss auf die Evaporationswahrscheinlichkeit bestimmt werden. Dazu werden - da es keine absolute Normierung gibt - zwei Faktoren benötigt, um diese Relationen zu quantifizieren - sprich D und K. Veranschaulicht wird dieses Verhalten in Abbildung 8.2.

Diese zwei Koeffizienten können nun durch Anpassung der Simulationen an die Messungen empirisch bestimmt werden. Dies ist mit genügender Genauigkeit durchführbar, wenn, wie in diesem Fall, in unterschiedlichen Kristallrichtungen verschiedene Ebenenanordnungen zur Verfügung stehen.

Man umgeht damit die Festlegung auf ein bestimmtes Evaporationsmodell bzw. auf einen bestimmten Evaporationsablauf und auch auf die genauen Einflüsse auf die Aktivierungsenergie Q. Stattdessen werden die energetischen Einflüsse und deren Auswirkungen nur empirisch und damit „aus der Messung heraus" bestimmt. Das bedeutet aber auch, dass man keine bzw. nur wenige Rückschlüsse auf das Evaporationsverhalten und weitere, beeinflussende Effekte ziehen kann. 


\subsection{Strukturanalyse an geordnetem ternären $\mathrm{Fe}_{3} \mathrm{Al}$}

\subsubsection{Allgemeine Analysen zur Platzbesetzung}

\section{APT-Messungen und -Simulationen}

Durch die systematischen Messungen in die drei Hauptrichtungen konnte für alle ternären Legierungen jeweils die Platzbesetzung der Zusätze bestimmt werden. Gerade für die chrom- und manganhaltigen Legierungen können dadurch bereits eindeutige Schlussfolgerungen zur $\beta$-Platzbesetzung gezogen werden. Die entsprechenden Simulationen bestätigen diese und liefern Abschätzungen des Ordnungsgrades.

Auch für die Legierung mit Vanadium kann durch die strukturierte Analyse von AtomVicinityDichteprofilen die $\beta$-Platzbesetzung ermittelt werden. Die Simulationen können diese Auswertungen im Wesentlichen lediglich qualitativ bestätigen. Sie zeigen damit auch auf, welche Grenzen der Simulationsalgorithmus und das zugrunde liegende Evaporationsmodell haben. Dies macht sich in den Peakformen von Eisen und Vanadium sehr deutlich bemerkbar und visualisiert damit die in 8.2.2 diskutierten Näherungen und Vereinfachungen der Simulation. Dennoch ist als positiver Aspekt festzuhalten, dass die Simulationen auch das Verhalten dieser Legierung wiedergeben können, das sich grundlegend von dem der chrom- und manganhaltigen Legierungen unterscheidet.

Die Platzbesetzung für die Legierung $\mathrm{Fe}_{3} \mathrm{Al}-\mathrm{Ti}$ kann als einzige nicht eindeutig allein anhand der AtomVicinity-Dichteprofile bestimmt werden. Hier hat sich die Möglichkeit zur Simulation als ein wesentliches Verfahren erwiesen, weitere Aussagen zu treffen. Insbesondere konnten mögliche Einflüsse des Al-Ti-Paarpotentials gut eingestuft werden. Damit scheint die $\beta$-Platzbesetzung auch für diese Legierung am konsistentesten, was auch allen Literaturangaben entspricht [17, 20, 23]. Außerdem bildet $\mathrm{Fe}_{3} \mathrm{Al}-\mathrm{Ti}$ in diesem Fall optimal gepackte Strukturen als LAVES-ähnliche-Phasen [15].

Das Ergebnis der $\beta$-Platzbesetzung der anderen Zusätze kann mit anderen Studien nur mit Einschränkungen verglichen werden, da es - wie in Kapitel 2 bereits geschildert - eine Vielzahl teilweise widersprechender Ergebnisse gibt. Allerdings kann hier die pauschale Annahme, dass Elemente links des Eisens im Periodensystem in der Regel auf $\beta$-Plätzen sitzen, für die vier untersuchten Zusätze bestätigt werden.

Insgesamt soll aber noch einmal eine wichtige Erkenntnis der APT-Untersuchungen betont werden: Die AtomVicinity-Dichteprofile können auch bei gleicher Kristallstruktur sehr stark von einander abweichen. Die Beispiele $\mathrm{Fe}_{3} \mathrm{Al}-\mathrm{Cr}$ und $\mathrm{Fe}_{3} \mathrm{Al}-\mathrm{V}$ zeigen dies am deutlichsten. Gerade mit der Kenntnis der $\beta$ Platzbesetzung wird klar, welche falschen Rückschlüsse gezogen werden können, wenn keine systematischen Analysen und/oder Simulationen durchgeführt werden. Würden beispielsweise bei diesen beiden Legierungen jeweils nur die Messungen in <111>-Richtung und deren Dichteprofile analysiert werden, so wäre von einer unterschiedlichen Platzbesetzung auszugehen. Dieses Beispiel zeigt also, welchen Einfluss die beteiligten Rekonstruktionseffekte auf APT-Daten haben können und wie aufmerksam diese dementsprechend analysiert werden müssen.

\section{ALCHEMI}

Die durchgeführten ALCHEMI-Untersuchungen können dieses Ergebnis insofern bestätigen, als dass für $\mathrm{V}, \mathrm{Cr}$ und $\mathrm{Mn}$ die $\alpha$-Platzbesetzung ausgeschlossen werden kann. Gerade die Besetzungsgrade von $\mathrm{Cr}$ und $V$ für $\beta / Y$-Plätze zeigen dies sehr deutlich. Auch wenn sie einen großen relativen Fehler aufweisen, so liegt die untere Grenze des Fehlerbereiches jeweils bei über $70 \% \beta / \gamma$-Platzbesetzung. Allein bei Mangan ist das Ergebnis weniger genau, mit 75\% $\pm 52 \%$ kann zwar eine $\alpha$-Platzbesetzung als unwahrscheinlicher betrachtet werden, sie ist aber bei einem solchen Fehler nicht auszuschließen. 
Diese relativ großen Ungenauigkeiten der ALCHEMI-Analysen können verschiedene Ursachen haben. Insbesondere Gitterdefekte, die den Channeling-Effekt stören, können auch bei geringer Konzentration bereits zu einer deutlichen Abschwächung desselbigen führen. Neben Leerstellen und Fremdatomen können gerade Antiphasengrenzen prinzipiell ein Problem darstellen [107]. Es ist daher anzunehmen, dass diese der Grund für nicht so eindeutige Ergebnisse sind. Zusätzlich ist dabei der Herstellungsprozess durch den fokussierten lonenstrahl zu bedenken. Auch wenn dadurch Proben definierter Geometrie angefertigt werden können, so kann eine oberflächliche Beschädigung des Materials nicht ausgeschlossen werden.

Daher konnten auch in einer zweiten ALCHEMI-Studie keine Ergebnisse gefunden werden, die Resultate sind im Anhang A zu finden. Bei dieser Messreihe wurden die EDX-Intensitäten abhängig vom Verkippungswinkel gemessen, so kann prinzipiell mit dem Vergleich zu theoretischen Berechnungen eine Platzbestimmung durchgeführt werden, die zwischen allen Kristallplätzen unterscheiden kann.

\subsubsection{Ordnungsgrade}

Durch den Vergleich von Messung und Simulation können, wie in 4.4 beschrieben, Ordnungsgrade ermittelt werden. Dabei werden sowohl Messunschärfe als auch Ordnungsgrad als veränderliche Simulationsparameter benutzt, die beide bis zur größtmöglichen Übereinstimmung zu optimieren sind. Die Übereinstimmung wird im Wesentlichen anhand des Verhältnisses Peak-Rauschsignal bestimmt. Die genaue Peakform bleibt aufgrund der in 8.2.2 beschriebenen Effekte in diesem Vergleich unberücksichtigt.

Messfehler ergeben sich dabei durch die allgemeine Mess- und Simulationsgenauigkeit, wie sie in 8.2.2 erörtert ist. Die dort beschriebenen Besonderheiten für die mittleren Peaks wurden umgangen, indem diese für die Optimierung der Übereinstimmung außer Acht gelassen wurden.

Insbesondere für die ternären Elemente ist die Bestimmung des Peak-Rauschsignal-Verhältnisses oftmals ungenauer, da sie nur geringe Konzentrationen aufweisen. Gerade bei eher kleinen gemessenen APT-Volumina treten entsprechend größere statistische Schwankungen auf. Diese sorgen dann folglich für eine weniger präzise Eingrenzung des Ordnungsgrades.

\begin{tabular}{ccc}
\hline Legierung & Ordnungsgrad $\xi_{\mathrm{v}}$ Aluminium [\%] & Ordnungsgrad $\xi_{\beta}$ ternäres Element [\%] \\
\hline $\mathrm{Fe}_{3} \mathrm{Al}$ & $90 \pm 5$ & -- \\
$\mathrm{Fe}_{3} \mathrm{Al}-\mathrm{Cr}$ & $94 \pm 2$ & $83 \pm 5$ \\
$\mathrm{Fe}_{3} \mathrm{Al}-\mathrm{Mn}$ & $94 \pm 5$ & $75 \pm 10$ \\
$\mathrm{Fe}_{3} \mathrm{Al}-\mathrm{V}$ & $93 \pm 7$ & $85 \pm 15$ \\
$\mathrm{Fe}_{3} \mathrm{Al}-\mathrm{Ti}$ & $92 \pm 4$ & $90 \pm 6$ \\
\hline
\end{tabular}

Tabelle 8.1: Ordnungsgrade ermittelt aus APT-Simulationen

Die ermittelten Ordnungsgrade für Aluminium und die ternären Zusätze jeder Legierung sind in Tabelle 8.1 aufgeführt. Für Vanadium konnten Ergebnisse nur mit größeren Ungenauigkeiten gefunden werden, da hier die Peakformen teilweise derart starke Unterschiede zeigen, dass auch insgesamt der Vergleich Messung-Simulation entsprechend undeutlich ist.

Es hat den Anschein, dass die Aluminiumordnung in Legierungen mit ternären Elementen ansteigt, allerdings kann dies im Rahmen der Messgenauigkeit nicht signifikant bestätigt werden. Auch in der ergänzenden Betrachtung von Literaturwerten zeigt sich ein solcher Trend nicht. Diese sind zum Ver- 
gleich in Abbildung 8.3 dargestellt, es handelt sich dabei um diejenigen Studien, bei denen die Ordnungsgrade quantitativ bestimmt wurden. Das sind Messungen durch Neutronenbeugung von ZUQING et al., MAGER et al. und KIM \& MORRIS [22-24], sowie die XRD-Untersuchungen von KRACHTOVIL et al. [12]. Alle gezeigten Werte gelten für eine Temperatur von $450^{\circ} \mathrm{C}$.

Die Ordnungsgrade von KRACHTOVIL et al. weichen deutlich von den anderen Ergebnissen ab. Hier zeigt sich, welche Ungenauigkeiten eine XRD-Untersuchung zur Unterscheidung der $\mathrm{DO}_{3}-$, $\mathrm{B} 2$ - und A2Struktur bei diesem Material aufweist (siehe auch 3.2.2 und Abbildung 3.4). Auch die Messung der $\beta$ Platzbesetzung von Mangan durch MAGER et al. zeigt eine geringe Abweichung. Dagegen kann für alle anderen Ergebnisse im Allgemeinen von einer guten Übereinstimmung zu den anderen Studien gesprochen werden. Gerade beim Ordnungsgrad von Aluminium zeigen sich deutlich gemeinsame Tendenzen, die jeweils im Bereich von über $90 \%$ liegen. Die Ordnungsgrade von Mangan zeigen eine gemeinsame Tendenz nach unten, d.h. sie sind jeweils merklich kleiner als die des Aluminiums und die der anderen Zusätze.

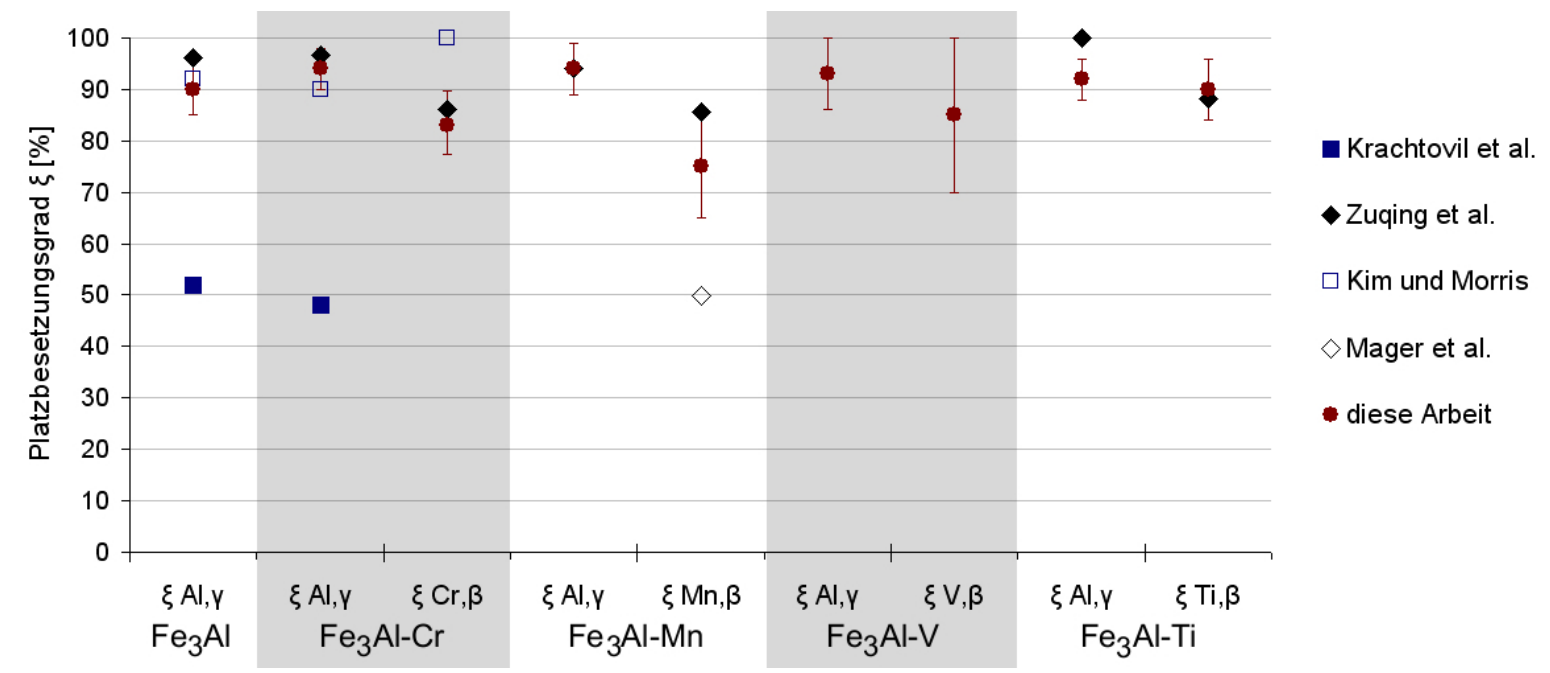

Abbildung 8.3: Ordnungsgrade in $\mathrm{Fe}_{3} \mathrm{Al}$ verschiedener Studien

Die durch diese Arbeit bestimmten Ordnungsgrade zeigen somit gute Übereinstimmung mit Literaturwerten, insbesondere mit Messungen mit Neutronenbeugung. Auch die Messgenauigkeit zeigt im Vergleich zu der Schwankungsbreite aller Messwerte ein zufriedenstellendes Ergebnis. Dies verdeutlicht, dass die AtomVicinity-Analyse von APT-Daten in Kombination mit APT-Simulationen ein nützliches Verfahren darstellt, um Ordnungsgrade zu ermitteln.

Bemerkenswert ist, dass die Werte dieser Arbeit einen sehr lokalen Ordnungsparameter darstellen. Denn durch die AtomVicinity-Analyse wird von jedem Al-Atom jeweils nur eine Umgebung von in diesem Fall 14Å Kantenlänge betrachtet. Damit bietet die hier konzipierte Analysemethode als einzige die Möglichkeit, Nahordnungseigenschaften im Bereich weniger Nanometer zu studieren.

\subsubsection{Zusammenhang mit mechanischen Eigenschaften}

In den APT-Messungen unterlagen die Zusätze Chrom und Mangan besonders der verfrühten Evaporation, was durch ihre geringere atomare Bindung hervorgerufen wurde. Die Zusätze sind auch diejenigen, die die Duktilität von $\mathrm{Fe}_{3} \mathrm{Al}$ erhöhen. Die Begründung dafür anhand des Absenkens der Antiphasengrenzenenergie steht mit diesen Beobachtungen in Einklang.

Die Abnahme der Raumtemperaturfestigkeit durch die betrachteten Zusätze wurde teilweise mit der Abnahme der $\mathrm{DO}_{3}$-Ordnung begründet [14]. Diese Annahme kann anhand des gemessenen Ordnungs- 
grades $\xi_{A, y}$ von Aluminium widerlegt werden, er zeigt keine Veränderung zu dem der binären Legierung. Folglich ist diese Veränderung der Festigkeit insbesondere auf atomare Wechselwirkungen zurückzuführen und nicht auf Ordnungsänderungen.

Anhand des Al-Ti-Potentials wurde festgestellt, dass Aluminium und Titan als nächste Nachbarn eine energetisch deutlich ungünstige Konstellation darstellen (5.4.2). Eine Folge davon könnte die erhöhte Sprödigkeit von $\mathrm{Fe}_{3} \mathrm{Al}-\mathrm{Ti}$ sein, da die Versetzungsbewegung durch diesen Effekt beeinträchtigt ist.

Die hohe Warmfestigkeit bei Zugabe von Titan und Vanadium ist zurückzuführen auf die feste Struktur und der erhöhte atomare Bindung, die sich auch in den APT-Messungen derart bemerkbar macht, dass diese Zusätze kaum der verfrühten Evaporation unterliegen. Dadurch bleibt die $\mathrm{DO}_{3}$-Ordnung auch für höhere Temperaturen stabil. Interessant ist hier das Ergebnis, dass sich diese Tatsache für die verwendete Auslagerungstemperatur von $450^{\circ} \mathrm{C}$ noch nicht auf den Grad der $\mathrm{DO}_{3}$-Ordnung auswirkt, dieser ist - bestimmt anhand des Ordnungsgrades $\xi_{A, \text {, }}$ von Aluminium wie oben beschrieben - für alle Legierungen annähernd gleich.

\subsection{Allgemeine Betrachtung der Messungen an nanokristallinem ternären $\mathrm{Fe}_{3} \mathrm{Al}$}

\subsubsection{Vorcharakterisierung durch XRD- und TEM-Messungen}

Die XRD-Messungen (3.2.2), die am MPIE GmbH in Düsseldorf durchgeführt wurden, zeigen aufgrund des Fehlens der charakteristischen $\mathrm{DO}_{3}$ - und $\mathrm{B} 2$-Röntgenpeaks, dass alle nanokristallinen Proben ungeordnet, d.h. in der A2-Struktur vorliegen. Es gibt keinen Hinweis auf weitere Phasen, wie z.B. Ausscheidungen. Die Korngrößenbestimmung anhand der Scherrer-Formel [59] führen zu Werten von 8nm bis $15 \mathrm{~nm}[60]$.

Diese Werte werden durch die TEM-Messungen bestätigt (7.1). Weiterhin zeigen sie, dass die Körner im Wesentlichen gleichmäßig geformt sind, d.h. ihre Ausmaße sind in alle Richtungen gleich. Dadurch kann keine ausgezeichnete Richtung der Gefügestruktur festgestellt werden, auch Beugungsaufnahmen geben keine Hinweise auf eine Anisotropie.

\subsubsection{Allgemeine Eigenschaften und Fortschritt der APT-Messungen}

Zunächst soll an dieser Stelle festgehalten werden, dass zum ersten Mal ein Einblick in die atomare Struktur von nanokristallinen Eisenaluminiden mithilfe der tomographischen Atomsonde gewonnen werden konnte. Dabei sind APT-Messungen dieses Materials im Allgemeinen schwierig aufgrund der ungünstigen Bedingungen - der hohen Sprödigkeit und der großen Anfälligkeit für Abrisse des nanokristallinen Materials.

Hauptsächlich verantwortlich für das Gelingen der Messungen im Rahmen dieser Arbeit ist die Probenpräparation, die auf hohe Probenfestigkeit optimiert wurde (siehe 3.2.2). Aber auch eine Optimierung der Messparameter ist dabei von Notwendigkeit. So zeigte sich, dass eine Anregung durch Spannungspulse eine derart hohe (zusätzliche) mechanische Belastung darstellt, dass die Wahrscheinlichkeit eines Abrisses sehr hoch ist. Daher konnten so nur zwei Proben erfolgreich gemessen werden, für alle anderen wurde eine Laseranregung benutzt. Diese hat den nachteiligen Effekt, dass dadurch die nominelle Auflösung aufgrund der zusätzlichen thermischen Anregung sinkt [47]. Des Weiteren musste auch die Messtemperatur erhöht werden, um die Abrisswahrscheinlichkeit zu senken, die nanokristallinen Proben wurden daher bei $70 \mathrm{~K}-80 \mathrm{~K}$ gemessen. 


\section{Messgenauigkeit/Auflösungsvermögen}

In allen Messungen können in den rekonstruierten APT-Volumina keine Kristallebenen erkannt werden. Das erschwert zum einen die Rekonstruktion, da die Eichung der Rekonstruktionsparameter $k_{f}$ und $\eta$ nur ungenauer anhand von Erfahrungswerten erfolgen kann. Zum anderen ist so auch keine Aussage über die genaue Ordnung bzw. Kristallstruktur möglich. Stattdessen muss hier die Analyse auf eine strukturelle Betrachtung mit einer Auflösung von ca. einem Nanometer beschränkt werden.

Eine Folge davon ist, dass dann auch keine Bereiche gleicher Ebenenorientierung - also Körner - bestimmt werden können. Auch durch Korngrenzensegregation oder -dichteabnahmen sind in den vorliegenden Messungen Körner nicht zu identifizieren, so dass die APT-Messungen eine Analyse z.B. von Korngrößen nicht zulassen.

Die Tatsache, dass Ebenen nicht aufgelöst werden können, überrascht zunächst. Die höhere Messtemperatur kann nicht der Grund sein, die APT-Messungen an massivem $\mathrm{Fe}_{3} \mathrm{Al}-\mathrm{Ti}$ bei 70K (5.4) zeigen klare Ebenenanordnungen. Da auch Messungen sowohl mit Laseranregung und Spannungsanregung durchgeführt wurden und auch drei verschiedene Atomsondenaufbauten verwendet wurden, sind diese Tatsachen ebenfalls nicht als systematische Ursache dafür zu erkennen.

Folglich ist es allem Anschein nach ein prinzipielles Problem, nanokristallines $\mathrm{Fe}_{3} \mathrm{Al}$ mit maximaler Auflösung - also erkennbaren Ebenen - mit einer Atomsonde zu messen. Ein möglicher Grund ist das ungleichmäßige Evaporationsverhalten aufgrund der hohen Defektdichte. Denkbar ist z.B. eine bevorzugte Feldverdampfung an Korngrenzen (Abbildung 8.4, roter Pfeil), gehemmte Feldverdampfung an Agglomeraten (blauer Pfeil) und eine allgemein ungleichmäßige Feldverdampfung an möglichen Antiphasengrenzen (grüner Pfeil) [46]. Dadurch werden Rekonstruktionseffekte wie die verfrühte/verspätete Evaporation hervorgerufen, sogar in einem noch größeren Maßstab als in Kapitel 4 und Kapitel 5 beschrieben. Außerdem werden dadurch die Trajektorien der Ionen verändert (schwarze Pfeile in Abbildung 8.4), was eine Fehlrekonstruktion begünstigt (lokaler Vergrößerungseffekt, siehe unten). Die Folge ist eine Verringerung der allgemeinen Auflösung um mehrere Ångström, was eine Ebenenauflösung ausschließt. Ein vergleichbares Verhalten wurde auch bei nanokristallinem Fe-Cu von WILLE beobachtet [53].

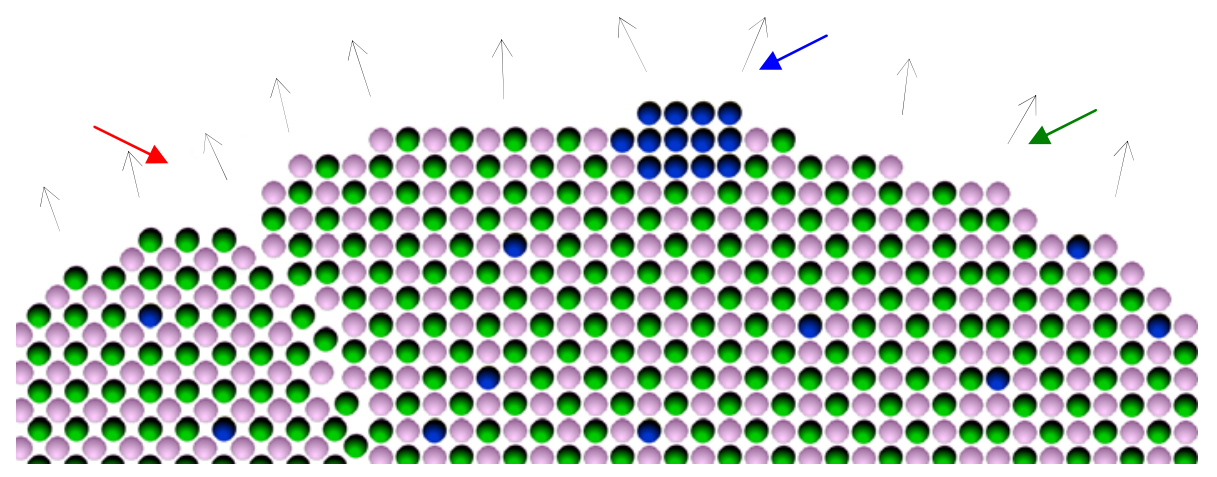

Abbildung 8.4: Schematische Feldevaporationsartefakte an einer APT-Spitze.

Schwarze Pfeile markieren Richtung der Feldverdampfung, farbige Pfeile siehe Text.

Sehr deutlich zeigen sich die Rekonstruktionseffekte bei den Agglomeraten, insbesondere bei $\mathrm{Fe}_{3} \mathrm{Al}-\mathrm{Ti}$ (Chargen 2 und 6). Wie die Konzentrationsprofile (Abbildung 7.9, Abbildung 7.19) zeigen, sinkt die Dichte im Bereich der Agglomerate auf bis zu ein Viertel ihres Wertes ab. Ein solches Verhalten ist unrealistisch und auch nicht durch unterschiedliche Kristallstrukturen oder veränderte Detektionsraten zu erklären. Stattdessen handelt es sich um ein Phänomen, das auf unterschiedliche Verdampfungsfeldstärken zurückzuführen ist und dem lokalen Vergrößerungseffekt entspricht $[48,100]$. Der Bereich mit erhöhter Titan- und/oder Kohlenstoffdichte zeigt die höhere Verdampfungsfeldstärke, dort versiegt die 
Evaporation eine Zeitlang und es entsteht eine Situation wie in Abbildung 8.4, blauer Pfeil. Im Endergebnis entstehen in der Rekonstruktion dann die gezeigten Artefakte (siehe auch [48, 108]). Zwei weitere Beobachtungen sprechen für das eben geschilderte Verhalten: Im Dichteprofil ist der Titan-Peak geringfügig weiter rechts als die Lücke des Eisens. Diese relative Verschiebung entspricht dem Effekt der Peakverschiebung aus Kapitel 4 und 5 aufgrund verspäteter Evaporation, allerdings wiederum in einer anderen Größenordnung. Als zweites kann beobachtet werden, dass die Titan/Kohlenstoffanhäufung deutlich größer ist, als das Loch in der Fe/Al-Matrix. Auch dies ist eine typische Erscheinung des lokalen Vergrößerungseffekts, die ihm letztlich auch den Namen gibt.

\subsection{Gefügeanalyse von nanokristallinem ternären $\mathrm{Fe}_{3} \mathrm{Al}$}

In der Betrachtung der APT-Messungen zeigt sich deutlich, dass die mechanisch legierten Proben eine durchweg inhomogene Struktur mit vielen, verschiedenartigen Agglomeraten zeigen. Die vor dem Mahlen eingeschmolzenen Proben zeigen hingegen ein anderes Gefüge. Es ist im Wesentlichen homogen und nur vereinzelt durch Oxideinschlüsse, Kohlenstoffanreicherungen oder Carbide unterbrochen. Die Ausnahme bildet hier $\mathrm{Fe}_{3} \mathrm{Al}-\mathrm{Ti}$, welches zwar eine homogene Matrix zeigt, aber ebenfalls ein regelmäßiges Auftreten von Titan-/Kohlenstoffanreicherungen.

\subsubsection{Verunreinigungen}

Zunächst soll das Auftreten von Kohlen- und Sauerstoff in den Proben diskutiert werden. Die Ausgangsmaterialien vor dem Kugelmahlen waren im Wesentlichen frei von diesen Elementen, sie sind also durch den Herstellungsprozess in das Material gekommen. Abbildung 8.5 zeigt dazu die ermittelten Konzentrationen in Abhängigkeit der drei verschiedenen Mahldauern. Die Kohlenstoffkonzentration $c_{C}$ steigt mit der Mahldauer annähernd proportional an. Man kann daher annehmen, dass der Kohlenstoff aus den Mahlkugeln und/oder dem Attritor durch Abrieb stammt, welcher mit der Mahldauer zunimmt.

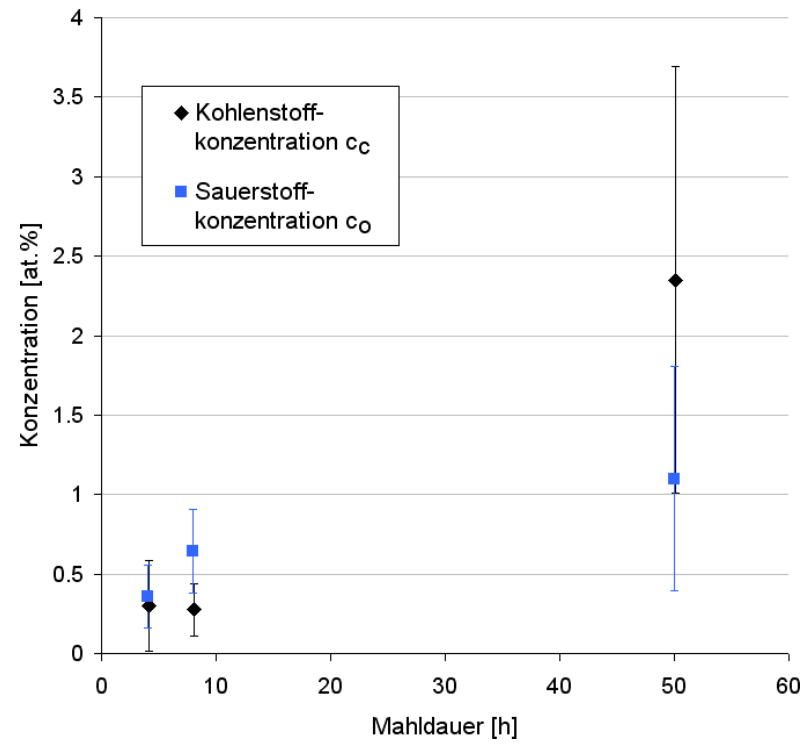

Abbildung 8.5: Kohlen- und Sauerstoffkonzentration in Abhängigkeit der Mahldauer
Die Sauerstoffkonzentration $c_{0}$ steigt ebenfalls mit der Mahldauer an, während sie bis $8 \mathrm{~h}$ Mahldauer jeweils über $c_{C}$ liegt, ist sie bei $50 \mathrm{~h}$ deutlich geringer. Man kann vermuten, dass der Sauerstoff eher aus vorhandenen Verunreinigungen stammt, z.B. Oxiden oder Adsorbaten an der Materialoberfläche und dann trotz zunehmender Mahldauer eine Sättigung einsetzt.

Zu betonen ist die Tatsache, dass Gallium nur mit Konzentrationen im Bereich von weniger als 0,1at\% detektiert wurde. Diese verschwindend geringe Menge bedeutet, dass während der Probenpräparation mit dem FIB quasi keine Galliumionen in den später gemessenen Bereich der Probe eindrangen und folglich keine strukturelle Veränderung durch die Probenherstellung erfolgte. 


\subsubsection{Homogenität}

Abbildung 8.6 zeigt die relativen Segregationsindizes $\mathrm{H}_{\mathrm{rel}}:=\mathrm{H} / \mathrm{H}_{\text {hom }}$ aller Messungen für Eisen und den ternären Zusatz, sie bestätigen die oben beschriebenen Beobachtungen. Für die eingeschmolzenen Proben mit Chrom, Mangan und Vanadium liegen sie bei allen betrachteten Bestandteilen unter oder sehr nahe bei 1 , entsprechen also dem Homogenitätskriterium. Bei den mechanisch legierten Proben zeigen sie höhere Werte über 1 und sind demnach statistisch inhomogen verteilt.

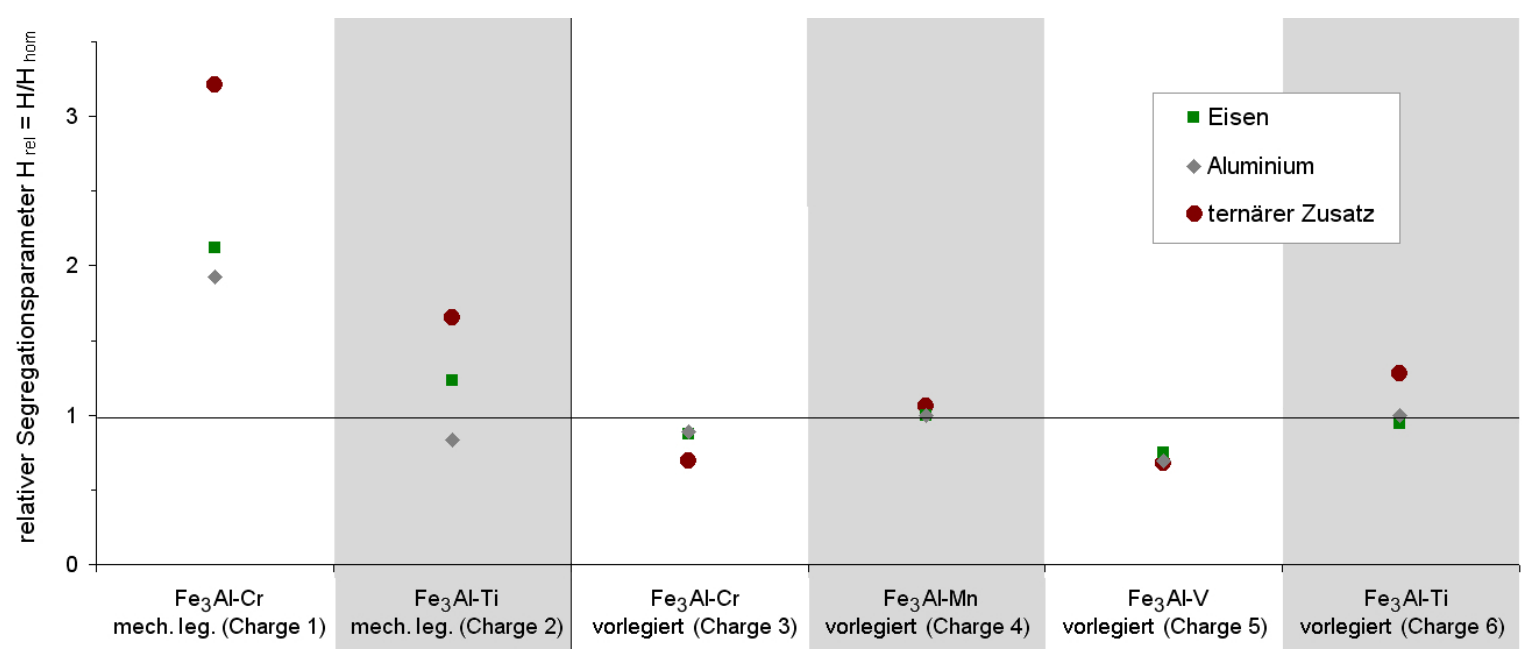

Abbildung 8.6: Segregationsindizes nanokristalliner Proben

Die eingeschmolzene $\mathrm{Fe}_{3} \mathrm{Al}$-Ti-Probe (Charge 6) hat Werte $\mathrm{H}_{\mathrm{Fe}}$ und $\mathrm{H}_{\mathrm{Al}}$ von unter $1, \mathrm{H}_{\mathrm{Ti}}$ liegt geringfügig darüber. Das ist überraschend, da diese Probe deutliche Titananhäufungen zeigt (Abbildung 7.18). Diese Segregationsparameter sind also ein Hinweis darauf, dass die Matrix um diese Agglomerate entsprechend homogen ist, was in der Gesamtstatistik zu diesen Werte führt.

\subsubsection{Korrelierte Segregation}

Abbildung 8.7 zeigt zusammenfassend die Ergebnisse der Kreuzkorrelationsanalyse, dargestellt sind die Mittelwerte von P für $\tau \gg$ 0. Die Werte mit Aluminium sind nicht verzeichnet, da es sich im Segregationsverhalten tendenziell sehr ähnlich wie Eisen verhält.

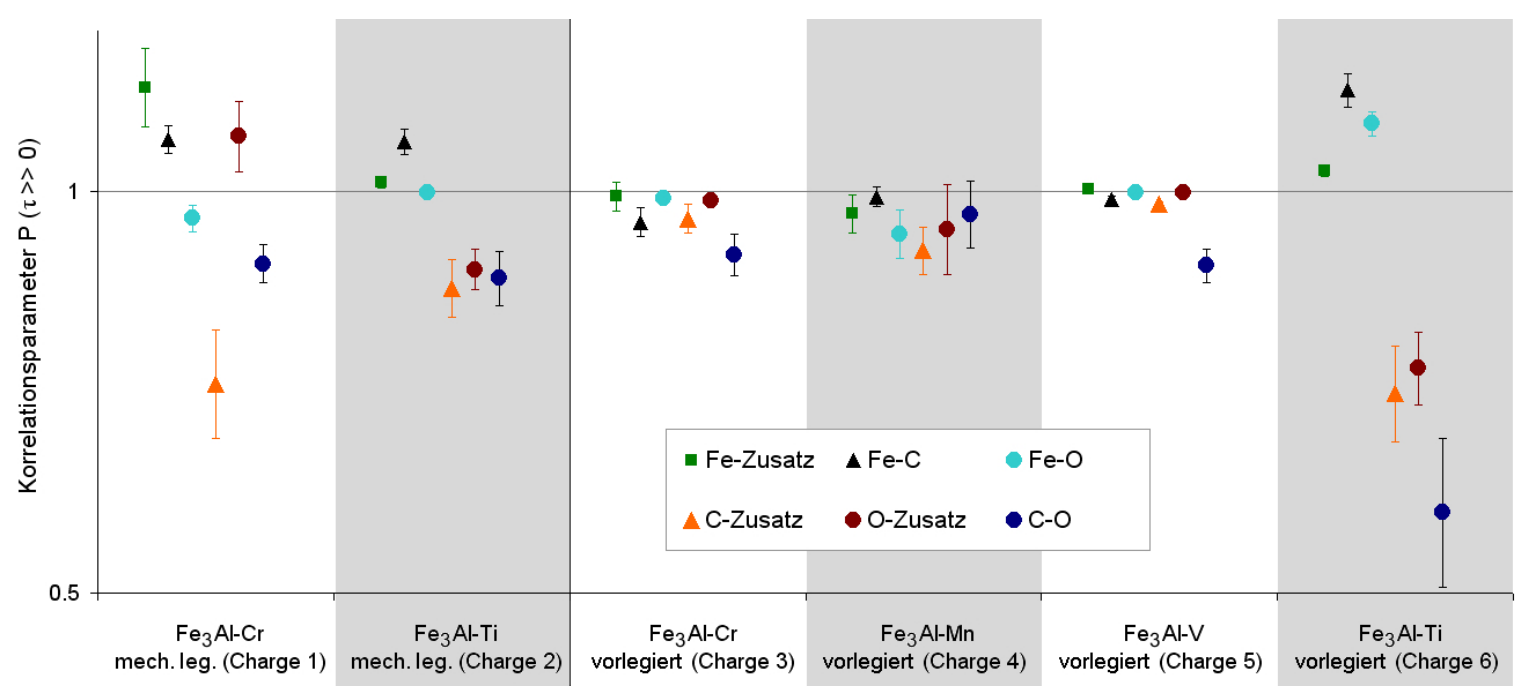

Abbildung 8.7: Übersicht der Kreuzkorrelationsanalysen an nanokristallinem ternären $\mathrm{Fe}_{3} \mathrm{Al}$ 
Es sind mehrere einzelne Beobachtungen zu machen:

- Eisen zeigt zu den Zusätzen nur in Charge 1 eine deutliche Segregation zu dem ternären Element (Chrom). Bei allen anderen Proben ist der Wert $\mathrm{P}_{\mathrm{Fe}-\text {-zusatz }}$ nahe bei 1.

- In den drei stark segregierten Probenchargen 1, 2 und 6 tritt Kohlenstoff verstärkt mit dem Zusatz auf (also Chrom bzw. Titan), bei den anderen tritt dieser Effekt nur tendenziell auf. Gleichzeitig ist er weniger im Bereich des Eisens zu finden. Eine Inhomogenität des Materials geht also mit einer beginnenden Carbidbildung des ternären Elementes einher, gleichzeitig separiert sich der Kohlenstoff von Eisen.

- Titan korreliert deutlich mit Sauerstoff, zeigt also ein gemeinsames Auftreten im Material. Die anderen ternären Zusätze zeigen hingegen kein (Chargen 3-5) oder ein unkorreliertes Auftreten zu Sauerstoff (Chrom, Charge 1).

- Kohlenstoff und Sauerstoff treten immer korreliert auf, was auf erhöhte Oxidation von Kohlenstoff hindeuten kann. Aber auch eine gleichzeitige, gemeinsame Verunreinigung des Probenmaterials könnte der Grund dafür sein.

Insgesamt zeigen also sowohl Chrom als auch Titan Anreicherungen zusammen mit Kohlenstoff, welche im Falle von Titan auch Oxidanreicherung beinhaltet. Allerdings muss man zwischen den mechanisch legierten und den vor dem Mahlen eingeschmolzenen Proben differenzieren:

Bei letzteren neigt Chrom nicht zur Carbidbildung, die Probe (Charge 3) ist nach Abbildung 8.6 vollkommen homogen und die Kreuzkorrelationsparameter mit Chrom sind alle nahe 1. Das in der $\mathrm{Fe}_{3} \mathrm{Al}-$ Matrix gelöste Chrom zeigt also keine Tendenz zur Entmischung. Gleiches Verhalten liegt auch für die Chargen 4 und 5 mit Mangan und Vanadium vor.

Die eingeschmolzene Probe mit Titan hingegen zeigt diese Entmischung. Deutlich ist hier die gemeinsame Separation von Ti, $\mathrm{C}$ und $\mathrm{O}$ zur restlichen Matrix anhand der Kreuzkorrelationsanalyse zu erkennen. Man kann daher vermuten, dass Titan am meisten von den vier Zusätzen zur Carbidbildung neigt und deswegen während der mechanischen Behandlung des Kugelmahlens bereits eine entsprechende Entmischung einsetzt. Dieses Verhalten entspricht Erkenntnissen von Übergangsmetallcarbiden, die im Allgemeinen eine hohe Festigkeit sowie eine hohe Schmelztemperatur aufweisen [109]. Für sie gilt generell, dass sie umso mehr zur Carbidbildung neigen, je weiter links sie im Periodensystem stehen $[110,111]$. Die Ursache liegt in den entsprechenden Werten der Bildungsenthalpie, die von Mangan zu Titan (in absoluten Zahlen) deutlich ansteigt, wie Tabelle 8.2 sie für Hochtemperaturreaktionen zeigt.

\begin{tabular}{cccc}
\hline Zusatz & Komposition & Schmelzpunkt $\left[^{\circ} \mathrm{C}\right]$ & Bildungsenthalpie $[\mathrm{kJ} / \mathrm{mol}]$ \\
\hline $\mathrm{Ti}$ & $\mathrm{TiC}$ & 3076 & $-92,9 \pm 8,9$ \\
$\mathrm{~V}$ & $\mathrm{~V}_{4} \mathrm{C}_{3}$ & 1320 & $-40,3 \pm 1,2$ \\
$\mathrm{Cr}$ & $\mathrm{Cr}_{7} \mathrm{C}_{3}$ & 1766 & $-14,1 \pm 0,8$ \\
$\mathrm{Mn}$ & $\mathrm{Mn}_{5} \mathrm{C}_{2}$ & 1171 & $-8,9 \pm 1,5$ \\
$\mathrm{Mn}$ & $\mathrm{Mn}_{7} \mathrm{C}_{3}$ & 1308 & $-9,1 \pm 1,3$ \\
$\mathrm{Fe}$ & $\mathrm{Fe}_{3} \mathrm{C}$ & 1252 & $4,7 \pm 1,1$
\end{tabular}

Tabelle 8.2: Eigenschaften von Übergangsmetallcarbiden nach [111] 
Titancarbid ist folglich eine derart stabile Phase, dass der während des Kugelmahlens eingearbeitete Kohlenstoff zur Entstehung dieser Agglomerate führt. Offenbar reichen die Bildungsenthalpien der anderen Zusätze nicht für eine entsprechende Entmischung aus.

Wie in 8.4.2 beschrieben, führen die titan- und kohlenstoffreichen Agglomerate in den APT-Messungen zu Rekonstruktionseffekten, da sie verspätet evaporieren, also eine höhere Feldverdampfungsfeldstärke aufweisen. Diese Beobachtung deckt sich mit den Erkenntnissen der hohen Stabilität und Festigkeit des Titancarbids.

\subsubsection{Gesamtbetrachtung des Gefüges}

An dieser Stelle soll aber auch noch einmal genauer diskutiert werden, wie diese Inhomogenitäten der Probenchargen 1, 2 und 6 zu charakterisieren sind. Intergranulare Konzentrationsschwankungen können ausgeschlossen werden, da es in jedem Konzentrationsprofil mehrere Bereiche gibt, die über mindestens $10 \mathrm{~nm}$ eine homogene, dem Mittelwert entsprechende Konzentrationsverteilung besitzen. Diese Bereiche entsprechen (mindestens) einem Korn, so dass innerhalb von Körnern im Allgemeinen von einer homogenen Verteilung ausgegangen werden kann.

Bei der Betrachtung der Ausmaße der Titanagglomerate wurden diese lateral auf $5 \mathrm{~nm}$ bis $10 \mathrm{~nm}$ (Abbildung 7.8, Abbildung 7.18) bestimmt. Aufgrund des lokalen Vergrößerungseffektes können diese Werte aber um bis zu 30\% zu groß gemessen worden sein [102]. In Messrichtung sind Ausmaße von $3 \mathrm{~nm}$ - 9nm zu erkennen. In 7.4 wurde aber bereits erläutert, dass aufgrund der Dichteschwankungen auch hier deutlich kleinere Werte anzunehmen sind. Zur Abschätzung der wahren Größe der Agglomerate können ebenfalls die in der Fe-Al-Matrix auftretenden „Löcher" beitragen, sie sind jeweils ca. 20\% kleiner als die Anhäufungen.

Es handelt sich also um abgeflachte/ellipsoide Agglomerationen weniger Nanometer Ausmaßes, die zu breit sind, um als Korngrenzensegregation bezeichnet zu werden. Dennoch kann man vermuten, dass diese Anhäufungen entlang von Korngrenzen entstehen.

Es ist nun zu diskutieren, warum sie dann eine gleichmäßige Ausrichtung zeigen. In den TEMUntersuchungen hat sich keine Vorzugsrichtung gezeigt, weder in den Beugungsbildern, noch in den Dunkelfeldaufnahmen. Die Ausscheidungen sind aber alle parallel zur Partikeloberfläche, welche herstellungsbedingt eine ausgezeichnete Richtung darstellt. Ebenfalls entspricht dies der APTMessrichtung, was wiederum zu einer Vorzugsrichtung in den Messungen führen kann. Der beschriebene lokale Vergrößerungseffekt kann dafür sorgen, dass beispielsweise runde Ausscheidungen abgeflacht rekonstruiert werden. Einige der in Abbildung 7.18 erkennbaren Agglomerate sind jedoch zu abgeflacht, um das Ergebnis dieser Fehlrekonstruktion zu sein. Es kann nicht ausgeschlossen werden, dass die Rekonstruktion insgesamt mit falschen Parametern erfolgte und daher der Eindruck flacher Anhäufungen entstehen.

Es ist aber auch möglich, dass die Agglomerate durchaus aufgrund der Herstellung eine bevorzugte Orientierung erhalten. Denkbar ist z.B., dass anfängliche Segregationen entlang von Korngrenzen stattfindet, was zu abgeflachten Agglomeraten führt. Möglicherweise sind diese stabiler, d.h. werden durch die Verformung des Materials weniger an der weiteren Entmischung gehindert, wenn sie parallel zur Oberfläche orientiert sind.

Gerade im Bezug zu mechanischen Eigenschaften ist das Auftreten dieser Agglomerate interessant. Zum einen, da Titan bei fortschreitender Entmischung nicht mehr innerhalb des $\mathrm{Fe}_{3} \mathrm{Al}$ gelöst ist. Um diesem Effekt entgegenzuwirken ist bei der Herstellung auf die Vermeidung von Kohlenstoffverunreinigungen zu achten. Zum anderen ist als positive Folge aber auch eine stabilisierende Wirkung auf die nanokristalline Struktur denkbar. Außerdem kann die Festigkeit durch diese Agglomerate ggf. gezielt 
gesteigert werden (vergleichbar zur Ausscheidungshärtung), entsprechende Folgestudien wären daher sehr interressant.

Abschließend soll noch einmal betont werden, dass sich diese Agglomerate im nanokristallinen $\mathrm{Fe}_{3} \mathrm{Al}$ nicht aufgrund der XRD und TEM-Messungen identifizieren ließen. Nur mithilfe der APT konnten diese Artefakte von wenigen Nanometern Größe erkannt werden. 


\section{Kapitel 9: Zusammenfassung}

In der vorliegenden Arbeit wurde geordnetes und nanokristallines $\mathrm{Fe}_{3} \mathrm{Al}$ mit den Zusätzen Chrom, Mangan, Vanadium und Titan hinsichtlich atomarer Strukturen untersucht. Dazu sind Platzbesetzung und Ordnungsgrade der massiven, $\mathrm{DO}_{3}$-geordneten Legierungen durch Atomsondentomografie bestimmt worden, deren genaue Analyse durch Simulationen von APT-Messungen unterstützt wurde. Von nanokristallinem Material sind durch APT-Untersuchungen die Gefügestruktur sowie atomare Homogenität und Segregation charakterisiert worden.

Um die Legierungen der $\mathrm{DO}_{3}$-geordneten Kristallstruktur zu untersuchen, wurden erstmals systematisch APT-Messungen orientiert in den drei kristallographischen Hauptrichtungen durchgeführt, um anhand von Dichteprofilen die jeweiligen Ebenenabfolgen zu ermitteln und daraus Aussagen über die Ordnung zu treffen. Die Atomsondentomografie lässt prinzipiell eine derartige Auflösung zu. Bei der Rekonstruktion der APT-Messungen kommt es jedoch zu Effekten, die durch die unterschiedlichen Feldverdampfungsfeldstärken der beteiligten Elemente und daraus folgender ungleichmäßiger Evaporation hervorgerufen werden. Dadurch sind die gemessenen, relativen Positionen der Ebenen und demzufolge auch ihre Abfolge verändert, was die Analysen zur Platzbesetzung deutlich erschwert.

Da diese Effekte nicht direkt umgangen werden können, wurden Simulationen von APT-Messungen durchgeführt, die die genannten Messartefakte berücksichtigen. Durch die Vergleiche gemessener APTDaten mit simulierten APT-Messungen verschiedener Kristallordnungen konnten so die Platzbesetzung ternärer Elemente und ihre Ordnungsgrade ermittelt werden. Ermöglicht wurde dies durch einen umfassenden Simulationsalgorithmus, der auf Prinzipien der Simulation von BoLL beruht. Neben der Verwendung eines umfangreicheren und genaueren Berechnungsmodells sorgte insbesondere die Anwendung des EAM-Formalismus zur Berechnung der Bindungsenergie für eine genauere Simulation. Mithilfe dieses etablierten und universelleren Modells konnten die deutlich kristallrichtungsabhängigen Fehlrekonstruktionen in guter Übereinstimmung zu den Messungen simuliert werden.

Für alle ternären Zusätze war es dadurch möglich, eine Besetzung der übernächsten Nachbarn des Aluminiums ( $\beta$-Plätze) in der $\mathrm{DO}_{3}$-Struktur zu belegen. Des Weiteren wurden die jeweiligen Ordnungsgrade für $\mathrm{Al}, \mathrm{Cr}, \mathrm{Mn}, \mathrm{V}$ und $\mathrm{Ti}$ bestimmt, die in guter Übereinstimmung zu Studien mit anderen Messmethoden stehen.

Auch ALCHEMI-Untersuchungen wurden durchgeführt, welche jedoch nur zu ungenaueren Messergebnissen hinsichtlich der Platzbesetzung führten. Diese bestätigen die APT- und Simulationsergebnisse dennoch tendenziell.

Des Weiteren wurden mithilfe der Simulationen die Fehlrekonstruktionen genau erörtert. So konnten neben der Verschiebung der Atompositionen auch der geometrische Einfluss des Messaufbaus quantifiziert werden. Insbesondere wurde gezeigt, wie unterschiedlich die APT-Rekonstruktionen verschiedener Legierungen mit gleichem Kristallaufbau aufgrund des unterschiedlichen Evaporationsverhaltens der Elemente sein können. Dadurch wird die Relevanz der APT-Simulationen verdeutlicht, um Fehlinterpretationen auszuschließen. Charakterisiert worden ist außerdem der Einfluss des unterschiedlichen Evaporationsverhaltens auf die Elementkonzentrationen, diese wurden mit EDX-Messungen überprüft und verglichen. 
Für nanokristallines, ternäres $\mathrm{Fe}_{3} \mathrm{Al}$ - welches durch Kugelmühlen hergestellt wurde - konnten erstmals APT-Messungen durchgeführt werden. Ermöglicht wurde dies durch eine optimierte Probenpräparation mithilfe des fokussierten Ionenstrahls. Die so hergestellten Proben wurden zunächst mit Transmissionselektronenmikroskopie untersucht um Korngrößen und -gefügestruktur zu analysieren. Die APTMessungen boten dann genauere Einblicke in die atomare Struktur. Damit konnte durch statistische Analysen die Homogenität und Segregation auf Nanometerskala sowie das korrelierte Auftreten von Elementen charakterisiert werden.

Bei den mechanisch legierten, chrom- bzw. titanhaltigen Proben wurden deutliche Abweichungen von einer homogenen Struktur festgestellt. Es zeigen sich einige Nanometer große Bereiche mit erhöhter Eisen- und Aluminiumkonzentration sowie ähnlich große Agglomerationen des ternären Elements und des Kohlenstoffs. Dieser erlangt als Verunreinigung eine Konzentration von 1at\% - 3at\% und ist - genau wie ebenfalls detektierter Sauerstoff - herstellungsbedingt. Kreuzkorrelationsanalysen zeigen, dass Chrom und Titan jeweils ein signifikant erhöhtes gemeinsames Auftreten mit Kohlenstoff besitzen, was als (Frühstadien in der) Entstehung von Carbiden begriffen werden kann.

Die vor dem Kugelmahlen eingeschmolzenen Proben zeigen hingegen eine homogene Struktur, in der keine Segregationen festgestellt werden können. Aufgrund der kürzeren Mahldauer weisen sie auch weniger Verunreinigungen auf. Hier können nur sehr vereinzelt Artefakte wie Oxideinschlüsse oder Kohlenstoffanreicherungen beobachtet werden. Eine deutliche Ausnahme bildet jedoch $\mathrm{Fe}_{3} \mathrm{Al}-4 \mathrm{at} \% \mathrm{Ti}$, welches gleichmäßig verteilte, ca. 3nm - 8nm große Titan-Kohlenstoffagglomerate aufzeigt. Diese können Anfänge der Präzipitation bzw. Entmischung von Titancarbiden sein, folglich neigt Titan von den vier Zusätzen am stärksten zur Carbidbildung in ternärem $\mathrm{Fe}_{3} \mathrm{Al}$.

Insgesamt wurden in dieser Arbeit durch systematische APT-Messungen genauere Einblicke in die atomare Struktur von hoch geordnetem und nanokristallinem, ternären $\mathrm{Fe}_{3} \mathrm{Al}$ gewonnen. Neben den $\mathrm{Er}$ kenntnissen zur Platzbesetzung, Ordnung, Homogenität und Gefügestruktur konnten auch die experimentellen Methoden - wie die Probenpräparation und insbesondere die Simulation von APTMessungen - erweitert werden. Bei letzteren ist mit dem EAM-Modell eine deutliche Verbesserung in der Berechnung von APT-Daten zu verzeichnen. Für weitere Optimierungen in diesem Bereich wäre es sinnvoll, z.B. den energetischen Einfluss des Feldes detaillierter, beispielsweise mithilfe eines FiniteElemente-Ansatzes, zu berechnen.

Für künftige Untersuchungen an $\mathrm{Fe}_{3} \mathrm{Al}$ ist insbesondere die Korrelation von mechanischen Eigenschaften zu den in dieser Arbeit erforschten Ordnungsphänomenen interessant. So könnte untersucht werden, welche Veränderungen der Struktureigenschaften auf Nanometerskala durch thermische und/oder mechanische Einflüsse entstehen und welche makroskopischen Auswirkungen diese haben. 


\section{Anhang A: Weitere ALCHEMI-Messungen}

\section{A.1. Theoretische Betrachtungen}

Eine umfangreichere Analyse zu der Methode aus Kapitel 6 besteht darin, die relativen EDXIntensitäten abhängig vom Einstrahlwinkel und der Platzbesetzung mithilfe der dynamischen Theorie der Elektronenbeugung zu berechnen. Durch Vergleich mit den entsprechenden Messungen kann dann auf Platzbesetzungen geschlussfolgert werden. Diese Herangehensweise ist ungemein schwieriger als das in Kapitel 6 geschilderte Zweistrahlverfahren, da neben der aufwändigen Berechnung der Blochwellenverteilung auch bestimmte Materialparameter bekannt sein müssen, insbesondere sind hier die Streufaktoren (Debye-Waller-Faktoren) der beteiligten Elemente zu nennen. Allerdings erlaubt diese Messmethode die Bestimmung der Platzbesetzungsgrade auch für mehr als zwei Kristallplätze. Ausführliche Beschreibungen über diese Rechnungen sind zu finden bei $[25,75,112]$.

Simulierte, schematische Intensitätsverläufe des EDX-Signals abhängig von der Platzbesetzung und des Einstrahlwinkels $\Theta$ (in Einheiten des Winkels $\Theta_{B}$ der Braggbedingung) zeigt Abbildung A.1. In diesem Beispiel für den Zusatz Chrom ist zu erkennen, dass sich die Signalverläufe für verschiedene Platzbesetzungen deutlich unterscheiden, so dass diese Analysen prinzipiell möglich sind.
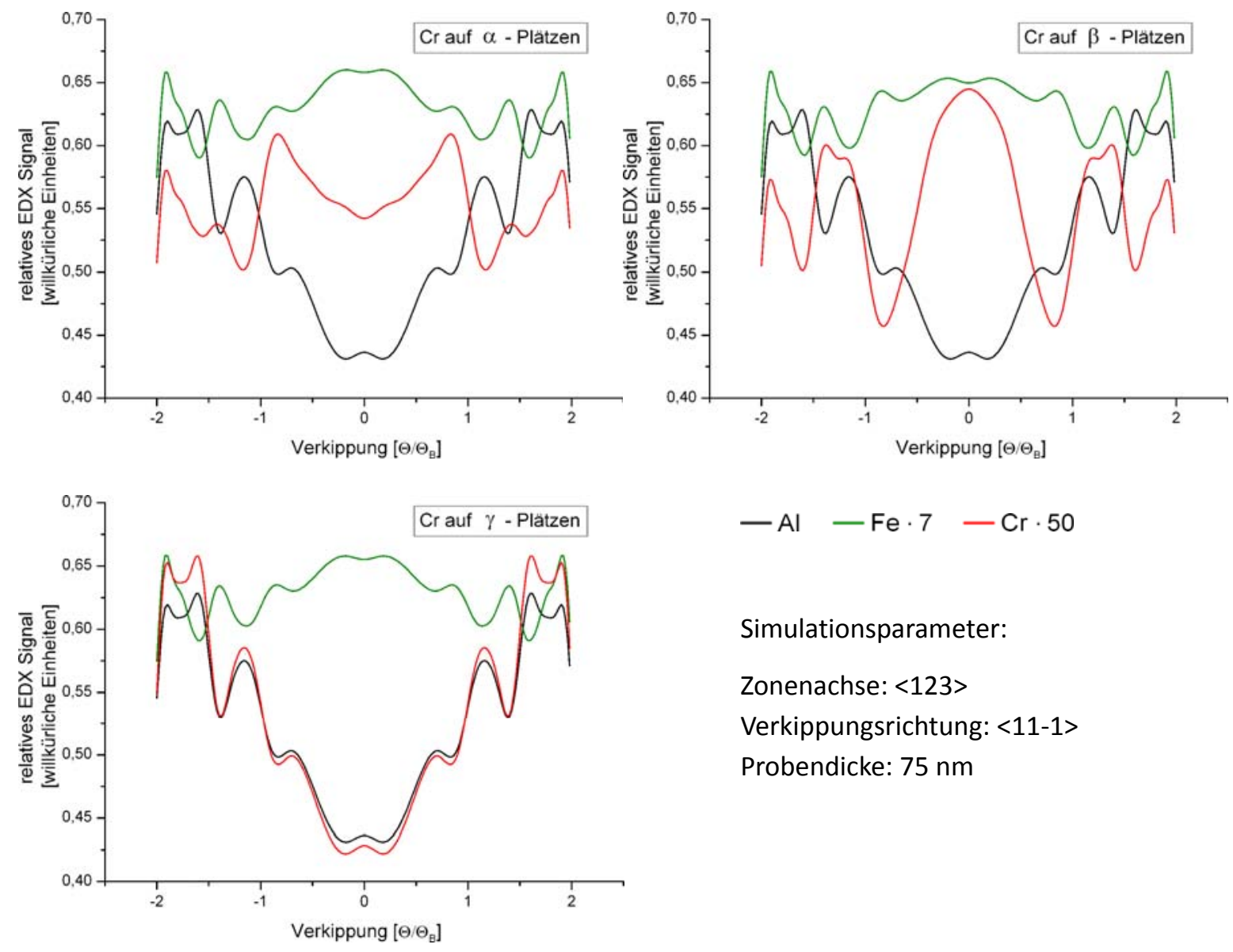

$-\mathrm{Al}-\mathrm{Fe} \cdot 7-\mathrm{Cr} \cdot 50$

Simulationsparameter:

Zonenachse: $<123>$

Verkippungsrichtung: $<11-1>$

Probendicke: $75 \mathrm{~nm}$

Abbildung A.1: Berechnete relative EDX-Intensitäten abhängig vom Verkippungswinkel für drei verschiedene Platzbesetzungen durch Chrom 


\section{A.2. Messresultate}

In den Messungen konnten jedoch keine Ergebnisse zur Ordnungsbestimmung erzielt werden. In einigen Proben ist ein Channelingeffekt gar nicht ausgemacht worden, eine kippwinkelabhängige Intensitätsveränderung war also nicht zu verzeichnen. Nur bei zwei Messungen sind signifikante Veränderungen des EDX-Signals gemessen worden, die entsprechenden Verläufe dieser chrom- bzw. vanadiumhaltigen Proben zeigt Abbildung A.2.
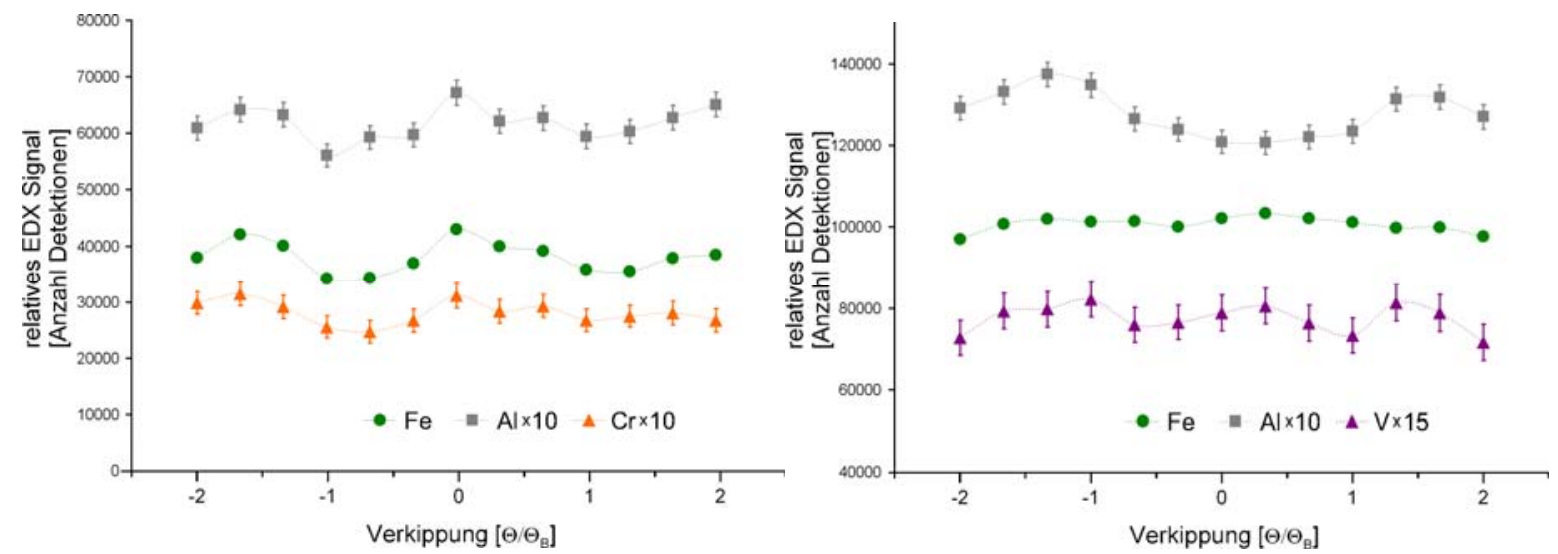

Abbildung A.2: ALCHEMI-Messungen von $\mathrm{Fe}_{3} \mathrm{Al}-\mathrm{Cr}$ und $\mathrm{Fe}_{3} \mathrm{Al}-\mathrm{V}$

Die gemessenen Verläufe von Aluminium und Eisen entsprechen nur mit deutlichen Abweichungen den theoretisch erwarteten. Die Verläufe der ternären Zusätze sind intuitiv keiner der drei berechneten Kurven zuzuordnen. Eine Tendenz zu einer bestimmten Platzbesetzung kann so nicht ausgemacht werden.

Um einen genauen Besetzungsgrad zu ermitteln, werden die gemessenen Kurven mit entsprechend relativem Anteil an die theoretischen Verläufe angefittet. Allerdings ist dies bei den vorliegenden Messdaten schon bei Aluminium und Eisen nicht mit einer zufriedenstellenden Signifikanz durchführbar. Für die ternären Zusätze ist es folglich - auch aufgrund des größeren relativen Fehlers - gar nicht möglich, diese Berechnungen derart durchzuführen, dass eine Aussage über die Platzbesetzung getroffen werden kann.

In dieser Messreihe liefert das ALCHEMI-Verfahren also keine verwertbaren Ergebnisse. Auch bei dem Zweistrahlmessverfahren sind nur ungenaue Resultate zu verzeichnen (Kapitel 6). Allem Anschein nach ist die Richtungsabhängigkeit der EDX-Intensität nicht sehr stark ausgeprägt, als Gründe sind insbesondere Gitterdefekte zu vermuten, dieser Aspekt wurde in 8.3.1 bereits diskutiert.

Es ist als Resultat daher nur festzuhalten, dass zur erfolgreicheren Anwendung der ALCHEMIMessmethode an ternärem $\mathrm{Fe}_{3} \mathrm{Al}$ Proben nötig sind, die eine ausgeprägtere, defektfreiere Kristallstruktur aufweisen. Folglich müsste dafür die Probenanfertigung optimiert werden. 


\section{Anhang B: Messdaten und Simulationsparameter}

\section{B.3. Materialparameter der Simulationen}

\begin{tabular}{|c|c|c|c|c|c|c|c|}
\hline & $\mathrm{Fe}$ & Al & $\mathrm{Cr}$ & $M n$ & $\mathbf{v}$ & $\mathrm{Ti}$ & Quellen \\
\hline $\begin{array}{l}\text { Sublimationsenergie } \\
E_{\text {sub }}[e V]\end{array}$ & 4,29 & 3,34 & 4,1 & 2,98 & 5,3 & 4,86 & [47] \\
\hline $\begin{array}{l}\text { Leerstellenbildungsener- } \\
\text { gie } E_{L S}[e V]\end{array}$ & 1,79 & 0,7 & 2,0 & 1,0 & 2,2 & 1,55 & $\begin{array}{c}{[72,88,} \\
113-117]\end{array}$ \\
\hline Atomvolumen $\Omega\left[\AA^{3}\right]$ & 11,820 & 16,608 & 12,045 & 12,207 & 13,862 & 17,727 & {$[47,118]$} \\
\hline $\begin{array}{l}\text { Elektronenbeitragskoeffi- } \\
\text { zient } \rho_{0}\left[\mathrm{eV}^{0,6} \AA^{-0,2}\right]\end{array}$ & 0,393 & 0,332 & 0,351 & 0,336 & 0,407 & 0,365 & {$[72]$} \\
\hline $\begin{array}{l}\text { Vorfaktor F der Einbet- } \\
\text { tungsfunktion }\left[\mathrm{eV}^{0,7} \AA^{0,1}\right]\end{array}$ & 0,81 & 1,26 & 0,85 & 0,82 & 1,12 & 0,91 & \\
\hline$N N-A b s t a n d d_{N N}[\AA]$ & 2,49 & 2,86 & 2,49 & 2,52 & 2,62 & 2,96 & {$[47,118]$} \\
\hline $\begin{array}{l}\text { I. lonisierungsenergie } \\
{[\mathrm{eV}]}\end{array}$ & 7,9 & 5,986 & 6,766 & 7,435 & 6,74 & 6,82 & [47] \\
\hline $\begin{array}{l}\text { II. lonisierungsenergie } \\
{[\mathrm{eV}]}\end{array}$ & 16,16 & 18,82 & 16,50 & 15,64 & 14,65 & 13,58 & [47] \\
\hline Austrittsarbeit $\Phi[\mathrm{eV}]$ & 4,4 & 4,1 & 4,6 & 3,8 & 4,1 & 4,0 & [47] \\
\hline
\end{tabular}

Tabelle B.1: Verwendete Berechnungsparameter für die APT-Simulation

\section{B.4. Messdaten der ALCHEMI (Zweistrahlmessverfahren)}

\begin{tabular}{lccccc}
\hline & & \multicolumn{4}{c}{ Intensität der $\mathrm{K}_{\alpha}$-Linie [Anzahl Detektionen] } \\
$\mathrm{Probe}$ & Element & Wert & Unbestimmtheit & Wert & Unbestimmtheit \\
\hline $\mathrm{Fe}_{3} \mathrm{Al}-\mathrm{Cr}$ & $\mathrm{Fe}$ & 77003569 & 2625120 & 74671087 & 2612350 \\
$\mathrm{Fe}_{3} \mathrm{Al}-\mathrm{Cr}$ & $\mathrm{Al}$ & 7507840 & 888555 & 11124178 & 1048640 \\
$\mathrm{Fe}_{3} \mathrm{Al}-\mathrm{Cr}$ & $\mathrm{Cr}$ & 4748769 & 672655 & 6511377 & 759017 \\
$\mathrm{Fe}_{3} \mathrm{Al}-\mathrm{Mn}$ & $\mathrm{Fe}$ & 71600740 & 2601420 & 71333897 & 2629480 \\
$\mathrm{Fe}_{3} \mathrm{Al}-\mathrm{Mn}$ & $\mathrm{Al}$ & 10795490 & 1146660 & 12807282 & 1185610 \\
$\mathrm{Fe}_{3} \mathrm{Al}-\mathrm{Mn}$ & $\mathrm{Mn}$ & 4961848 & 745368 & 5488767 & 805540 \\
$\mathrm{Fe}_{3} \mathrm{Al}-\mathrm{V}$ & $\mathrm{Fe}$ & 54337656 & 2285620 & 106734432 & 3157550 \\
$\mathrm{Fe}_{3} \mathrm{Al}-\mathrm{V}$ & $\mathrm{Al}$ & 14115358 & 1314370 & 19687103 & 1522380 \\
$\mathrm{Fe}_{3} \mathrm{Al}-\mathrm{V}$ & $\mathrm{V}$ & 3377926 & 601659 & 4672853 & 644668 \\
\hline
\end{tabular}

Tabelle B.2: Messdaten der ALCHEMI (Zweistrahlverfahren) 


\section{Anhang C: Abschätzungen}

\section{C.1. Einfluss der Projektion auf Atompositionen der APT-Simulation}

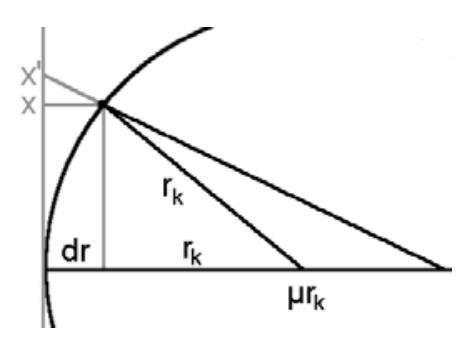

Abbildung C.1: Geometrische Verhältnisse der Projektion bei der APT

$$
\mathrm{x}=\frac{\mathrm{x}_{\mathrm{det}}}{\mathrm{M}}=\mathrm{x}_{0}
$$

Wie in 3.3.4 beschrieben, wurde bei der Simulation im Rahmen dieser Arbeit der Projektionseffekt mit einkalkuliert. Anschaulich würde das bedeuten, ein evaporierendes Atom bewegt sich nach der Ablösung nicht in Messrichtung, sondern in einem steileren Winkel zur Oberfläche (siehe Abbildung C.1). Da bei der Simulation mit einer Vergrößerung von $\mathrm{M}=1$ gerechnet wird, liegt der Detektor damit quasi in der Zwischenebene (vergleiche Abbildung 3.2). Die ursprünglichen Atompositionen seien $x_{0}, y_{0}$ und $z_{0}$, die berechneten ohne Projektion $x, y$ und $z$ und diejenigen mit Projektion $x^{\prime}, y^{\prime}$ und $z^{\prime}$.

Ohne Projektion gilt:

$$
z=r_{k}-\sqrt{r_{k}^{2}-\left(\frac{x_{d e t}}{M}\right)^{2}-\left(\frac{y_{d e t}}{M}\right)^{2}}=r_{k}-\sqrt{r_{k}^{2}-x_{0}^{2}-y_{0}^{2}}
$$

Mit Projektion muss zunächst dr bestimmt werden, um dann den Strahlensatz zu verwenden:

$$
d r=r_{k}-\sqrt{r_{k}^{2}-x_{0}^{2}-y_{0}^{2}} \quad \frac{x^{\prime}}{x}=\frac{\mu \cdot r_{k}}{\mu \cdot r_{k}-r_{k}+\sqrt{r_{k}^{2}-x_{0}^{2}-y_{0}^{2}}}
$$

Daraus folgt:

$$
\begin{aligned}
& x^{\prime}=\frac{\mu \cdot r_{k} \cdot x_{0}}{\mu \cdot r_{k}-r_{k}+\sqrt{r_{k}^{2}-x_{0}^{2}-y_{0}^{2}}} \\
& z^{\prime}=r_{k}-\sqrt{r_{k}^{2}-x^{\prime 2}-y^{\prime 2}}=r_{k}-\sqrt{r_{k}^{2}-\left(\frac{\mu \cdot r_{k} \cdot x_{0}}{\mu \cdot r_{k}-r_{k}+\sqrt{r_{k}^{2}-x_{0}^{2}-y_{0}^{2}}}\right)^{2}-\left(\frac{\mu \cdot r_{k} \cdot y_{0}}{\mu \cdot r_{k}-r_{k}+\sqrt{r_{k}^{2}-x_{0}^{2}-y_{0}^{2}}}\right)^{2}}
\end{aligned}
$$

Mit $r_{k}=500 \AA, x_{0}=100 \AA, y_{0}=100 \AA$ und $\mu=1,5$ ergibt sich ohne Projektion:

$$
x=x_{0}=100 \AA \quad y=y_{0}=100 \AA \quad z=20,42 \AA
$$

Mit Projektion erhält man:

$$
x=102,80 \AA \quad y=102,80 \AA \quad z=21,60 \AA
$$




\section{C.2. APT-Fehlrekonstruktion durch Unterschiede in Feldverdampfungs- feldstärken zweier Elemente}

In 4.1 wurde beschrieben, dass es in einer Legierung mit zwei Elementen mit unterschiedlichen Feldverdampfungsfeldstärken bei einem Element zu einer verfrühten Evaporation kommen kann, als Extremfall gar zu einer korrelierte Evaporation zweier Atome (siehe 4.5). Dabei wurde die z-Komponente um bis zu $1,5 \AA$ (in <100>-Orientierung) zu klein rekonstruiert.

An dieser Stelle soll der Fehler für die laterale Position bestimmt werden. Dazu muss zunächst der Apexradius $r_{k}{ }^{\prime}$ bestimmt werden, wenn keine verfrühte Evaporation stattfände. Das Atom würde also erst desorbieren, wenn die Spitze 1,5 $\AA$ kürzer geworden wäre. Mit $r_{k}=500 \AA$ und einem Probenschaftwinkel von $20^{\circ}$ gilt:

$$
r_{k}^{\prime}=r_{k}+1,5 \AA \cdot \tan \left(20^{\circ}\right) \approx r_{k}+0,5 \AA=r_{k} \cdot 1,001
$$

Für die Vergrößerung und die rekonstruierte Position $x^{\prime}$ bedeutet das:

$$
M^{\prime}=\frac{L}{\eta \cdot r_{k}^{\prime}}=\frac{M}{1,001} \quad x^{\prime}=\frac{x_{d e t}}{M^{\prime}} \approx \frac{1,001 \cdot x_{d e t}}{M} \cdot=1,001 \cdot x
$$

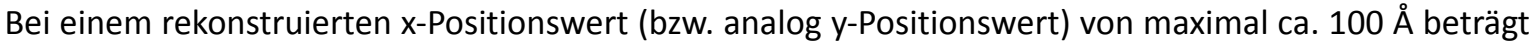
der Fehler also etwa 0,1 $\AA$, was deutlich unterhalb der lateralen Auflösung von ca. $2 \AA$ liegt und sich auch nicht in den AtomVicinity-Dichteprofilen bemerkbar machen würde. [46, 48]. 


\section{Literaturverzeichnis}

[1] N.S. Stoloff: Iron aluminides: present status and future prospects, Mater. Sci. Eng. A. 258 (1998) S.1-14.

[2] C.G. McKamey, J.H. DeVan, P.F. Tortorelli, V.K. Sikka: A review of recent developments in $\mathrm{Fe}_{3} \mathrm{Al}$ based alloys, Journal of Materials Research. 6 (1991) S.1779-1805.

[3] C.G. McKamey, J.A. Horton, C.T. Liu: Effect of chromium on room temperature ductility and fracture mode in $\mathrm{Fe}_{3} \mathrm{Al}$, Scr. Metall. 22 (1988) S.1679-1681.

[4] L. Baiyang, X. Zhang, L. Maosen, Z. Zhang: Effects of $\mathrm{Mn}$ on fracture behaviour of $\mathrm{DO}_{3} \mathrm{Fe}_{3} \mathrm{Al}$ based intermetallic alloy, Journal of Material Science. 33 (1998) S.1481-1486.

[5] Y. Nishino, C. Kumada, S. Asano: Phase stability of $\mathrm{Fe}_{3} \mathrm{Al}$ with addition of $3 d$ transition elements, Scripta Materialia. 36 (1997) S.461-466.

[6] L. Anthony, B. Fultz: Effects of early transition metal solutes on the $\mathrm{DO}_{3}-\mathrm{B} 2$ critical temperature of $\mathrm{Fe}_{3} \mathrm{Al}$, Acta Metallurgica Et Materialia. 43 (1995) S.3885-3891.

[7] M. Rafiei, M.H. Enayati, F. Karimzadeh: The effect of Ti addition on alloying and formation of nanocrystalline structure in Fe-Al system, J Mater Sci. 45 (2010) S.4058-4062.

[8] G. Frommeyer, C. Derder, J.A. Jimenéz: Superplasticity of $\mathrm{Fe}_{3} \mathrm{Al}(\mathrm{Cr})$, Material Science and Technology. 18 (2002) S.981 - 986.

[9] P.J. Black: The structure of FeAl 3. I, Acta Cryst. 8 (1955) S.43-48.

[10] T.B. Massalski: Binary Alloy Phase Diagrams, 2. Aufl., ASM International (1990).

[11] F. Král, P. Schwander, G. Kostorz: Superdislocations and antiphase boundary energies in deformed $\mathrm{Fe}_{3} \mathrm{Al}$ single crystals with chromium, Acta Materialia. 45 (1997) S.675-682.

[12] P. Kratochvíl, J. Kopecek, J. Pesicka: Order of $\mathrm{Fe}_{3} \mathrm{Al}$ alloys and their yield stress, Materials Science and Engineering A. 234-236 (1997) S.142-144.

[13] Y. Nishino, Y. Makino: Effect of vanadium substitution on strength properties of $\mathrm{Fe}_{3} \mathrm{Al}$-based alloys, Materials Science and Engineering A. 319-321 (2001) S.368-371.

[14] S.-M. Zhu, K. Sakamoto, M. Tamura, K. Iwasaki: Effects of titanium addition on the microstructure and mechanical behavior of iron aluminide $\mathrm{Fe}_{3} \mathrm{Al}$, Japan Institute of Metals. 42 (2001).

[15] I.S. Golovin: Anelastic relaxation in ternary Fe-Al-Me alloys: $\mathrm{Me}=\mathrm{Co}, \mathrm{Cr}, \mathrm{Ge}, \mathrm{Mn}, \mathrm{Nb}, \mathrm{Si}, \mathrm{Ta}, \mathrm{Ti}, \mathrm{Zr}$, Materials Science and Engineering: A. 442 (2006) S.92-98.

[16] E. Popiel, M. Tuszynski, W. Zarek, T. Rendecki: Investigation of $\mathrm{Fe}_{3-x} \mathrm{~V}_{x} \mathrm{Al}$ alloys with $\mathrm{DO}_{3}$ type structure by X-ray, magnetostatic and Mössbauer effect methods, Journal of the Less Common Metals. 146 (1989) S.127-135.

[17] R.T. Fortnum, D.E. Mikkola: Effects of molybdenum, titanium and silicon additions on the $\mathrm{DO}_{3}<-$ $>$ B2 transition temperature for alloys near $\mathrm{Fe}_{3} \mathrm{Al}$, Materials Science and Engineering. 91 (1987) S.223-231.

[18] D. Satula, L. Dobrzynski, A. Malinowski, K. Szymanski, u. a.: Hyperfine fields, local environment effects and site preference in $\mathrm{Fe}_{3-x} \mathrm{Cr}_{x} \mathrm{Al}$ alloys, Journal of Magnetism and Magnetic Materials. 151 (1995) S.211-220.

[19] D. Satula, L. Dobrzynski, J. Waliszewski, K. Szymanski, u. a.: Structural and magnetic properties of $\mathrm{Fe}$-Cr-Al alloys with $\mathrm{DO}_{3}$-type structure, Journal of Magnetism and Magnetic Materials. 169 (1997) S.240-252.

[20] G. Athanassiadis, G. Le Caer, J. Foct, L. Rimlinger: Study of ternary ordered solid solutions derived from $\mathrm{Fe}_{3} \mathrm{Al}$ by Substitution, Phys. Stat. Sol. (a). 40 (1977) S.425-435. 
[21] W. Yan: ALCHEMI study of $\mathrm{Cr}$ atoms in $\mathrm{Fe}_{3} \mathrm{Al}$ alloy, Journal of Xian Institute of Technology. 15 (1995) S.1-9.

[22] S.M. Kim, D.G. Morris: Long range order and vacancy properties in Al-rich $\mathrm{Fe}_{3} \mathrm{Al}$ and $\mathrm{Fe}{ }_{3} \mathrm{Al}(\mathrm{Cr})$ alloys, Acta Materialia. 46 (1998) S.2587-2602.

[23] S. Zuqing, Y. Wangyue, S. Lizhen, H. Yuanding, u. a.: Neutron diffraction study on site occupation of substitutional elements at sub lattices in $\mathrm{Fe}_{3} \mathrm{Al}$ intermetallics, Materials Science and Engineering A. 258 (1998) S.69-74.

[24] S. Mager, E. Wieser, T. Zemčík, O. Schneeweiss, u. a.: Investigations of the magnetic moments in $\left(F e_{1-x} M n_{x}\right)_{3} A l$ alloys, Phys. Stat. Sol. (a). 52 (1979) S.249-258.

[25] I.M. Anderson: ALCHEMI study of site distributions of 3d-transition metals in B2-ordered iron aluminides, Acta Materialia. 45 (1997) S.3897-3909.

[26] N. Lakshmi, K. Venugopalan, J. Varma: Hyperfine-field studies of $\mathrm{Fe}_{3} \mathrm{Al}$ and $\mathrm{Fe}_{3-x} \mathrm{Cr}_{3} \mathrm{Al}$ alloys, Physical Review B. 47 (1993) S.14054-14061.

[27] B.V. Reddy, D.H. Sastry, S.C. Deevi, S.N. Khanna: Magnetic coupling and site occupancy of impurities in $\mathrm{Fe}_{3} \mathrm{Al}$, Phys. Rev. B. 64 (2001) S.224419.

[28] D. Fuks, S. Dorfman, V. Liubich, L. Kutsenko: Site preference occupation of $\mathrm{Ti}$, Ni, and $\mathrm{Cr}$ in $\mathrm{Fe}_{3} \mathrm{Al}$ compound, Int. J. Quantum Chem. 90 (2002) S.1478-1490.

[29] C.L. Fu, J. Zou: Site preference of ternary alloying additions in FeAl and NiAl by first-principles calculations, Acta Materialia. 44 (1996) S.1471-1478.

[30] N.I. Medvedeva, Y.N. Gornostyrev, D.L. Novikov, O.N. Mryasov, u. a.: Ternary site preference energies, size misfits and solid solution hardening in NiAl and FeAl, Acta Materialia. 46 (1998) S.3433-3442.

[31] J. Cai, S. Liu, Y. Lou, B. Ma, u. a.: Site Occupancy of Alloying Elements and Their Effects on the $\mathrm{DO}_{3}$ Phase Stability in $\mathrm{Fe}_{3} \mathrm{Al}$, J. Mater. Sci. Technol. 15 (1999) S.339.

[32] A. Go, M. Pugaczowa-Michalska, L. Dobrzynski: Electronic structure and the site preference of chromium in $\mathrm{Fe}_{3} \mathrm{Al}$, Physica Status Solidi (B). 243 (2006) S.1241-1247.

[33] E. Bonetti, G. Scipione, R. Frattini, S. Enzo, u. a.: Structural and elastic behavior of $\mathrm{Fe}_{50} \mathrm{Al}_{50} \mathrm{na}^{-}$ nocrystalline alloys, J. Appl. Phys. 79 (1996) S.7537 - 7544.

[34] C. Mangler, C. Gammer, H.P. Karnthaler, C. Rentenberger: Structural modifications during heating of bulk nanocrystalline FeAl produced by high-pressure torsion, Acta Mater. 58 (2010) S.5631-5638.

[35] Y.B. Pithawalla, S.C. Deevi, M.S. El-Shall: Preparation of ultrafine and nanocrystalline FeAl powders, Materials Science and Engineering A. 329-331 (2002) S.92-98.

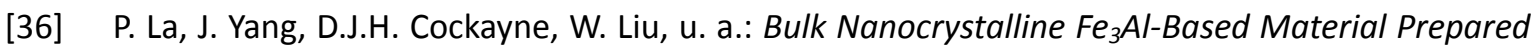
by Aluminothermic Reaction, Adv. Mater. 18 (2006) S.733-737.

[37] S. Sarkar, C. Bansal: Atomic disorder-order phase transformation in nanocrystalline Fe-Al, Journal of Alloys and Compounds. 334 (2002) S.135-142.

[38] A.J. Melmed, N.C. Tambakis, M. Lau, E.J. Lavernia: APFIM study of impurities in nanocrystalline Fe-Al alloy, Progress in Surface Science. 59 (1998) S.313-321.

[39] P. La, Y. Wei, R. Lv, Y. Zhao, u. a.: Effect of Mn element on microstructure and mechanical properties of bulk nanocrystalline $\mathrm{Fe}_{3} \mathrm{Al}$ based materials prepared by aluminothermic reaction, Materials Science and Engineering: A. 527 (2010) S.2313-2319.

[40] E. Bonetti, G. Scipione, G. Valdre, S. Enzo, u. a.: A study of nanocrystalline iron and aluminium metals and $\mathrm{Fe}_{3} \mathrm{Al}$ intermetallic by mechanical alloying, J Mater Sci. 30 (1995) S.2220-2226.

[41] M.A. Morris-Muñoz, A. Dodge, D.G. Morris: Structure, strength and toughness of nanocrystalline FeAl, Nanostructured Materials. 11 (1999) S.873-885. 
[42] E. Jartych, J.K. Zurawicz, D. Oleszak, M. Pekala: Magnetic properties and structure of nanocrystalline Fe-Al and Fe-Ni alloys, Nanostructured Materials. 12 (1999) S.927-930.

[43] R.A. Varin, J. Bystrzycki, A. Calka: Characterization of nanocrystalline Fe-45 at\% Al intermetallic powders obtained by controlled ball milling and the influence of annealing, Intermetallics. 7 (1999) S.917-930.

[44] R. Esparza, G. Rosas, J.A. Ascencio, R. Pérez: Effects of Minor Element Additions to the Nanocrystalline FeAl Intermetallic Alloy Obtained by Mechanical Alloying, Materials and Manufacturing Processes. 20 (2005) S.823.

[45] V. Sebastian, N. Lakshmi, K. Venugopalan: Mössbauer study of incompletely alloyed nanocrystalline $\mathrm{Fe}_{100-x} \mathrm{Al}_{x}$ prepared by high energy ball milling, Hyperfine Interact. 174 (2007) S.127-135.

[46] T. Al-Kassab, H. Wollenberger, G. Schmitz, R. Kirchheim: Tomography by atom probe field ion microscopy, in: High-Resolution Imaging and Spectrometry of Materials, Springer Verlag GmbH, Berlin (2003) S. 271-320.

[47] M.K. Miller: Atom Probe Tomography: Analysis at the Atomic Level, 1. Aufl., Springer Verlag $\mathrm{GmbH}$ (2000).

[48] M.K. Miller, G.D.W. Smith: Atom Probe Microanalysis: Principles and Applications to Materials Problems, Materials Research Society (1989).

[49] P. Bas, A. Bostel, B. Deconihout, D. Blavette: A general protocol for the reconstruction of $3 D$ atom probe data, Applied Surface Science. 87-88 (1995) S.298-304.

[50] D. Blavette, B. Déconihout, S. Chambreland, A. Bostel: Three-dimensional imaging of chemical order with the tomographic atom-probe, Ultramicroscopy. 70 (1998) S.115-124.

[51] C. Booth-Morrison, Z. Mao, R.D. Noebe, D.N. Seidman: Chromium and tantalum site substitution patterns in $\mathrm{Ni}_{3} \mathrm{Al}\left(\mathrm{L1}_{2}\right) \gamma^{\prime}$-precipitates, Appl. Phys. Lett. 93 (2008) S.033103.

[52] T. Boll, T. Al-Kassab, Y. Yuan, Z.G. Liu: Investigation of the site occupation of atoms in pure and doped TiAl/Ti ${ }_{3} \mathrm{Al}$ intermetallic, Ultramicroscopy. 107 (2007) S.796-801.

[53] C. Wille: Investigation of the segregation behaviour in nanocrystalline materials, Dissertation, Georg-August-Universität Göttingen(2009).

[54] U. Krengel: Einführung in die Wahrscheinlichkeitstheorie und Statistik. Für Studium, Berufspraxis und Lehramt, 7. Aufl., Vieweg (2005).

[55] E.M. Hoover: An introduction to regional economics, 2. Aufl., Knopf, New York (1975).

[56] Ungleichverteilungsmaß, http://de.wikipedia.org/wiki/Ungleichverteilungsmaß, Zugriff am 18. April 2011.

[57] I.N. Bronstein, K.A. Semendjajew, G. Musiol, H. Mühlig: Taschenbuch der Mathematik, 5. Aufl., Harri Deutsch (2000).

[58] G. Frommeyer, Z.G. Liu, J. Wesemann, N. Wanderka: Investigations on field-ion image formation and field evaporation sequences of $\mathrm{DO}_{3}$-ordered $\mathrm{Fe}_{3} \mathrm{Al}$., Ultramicroscopy. 92 (2002) S.57.

[59] P. Scherrer: Göttinger Nachrichten. 2 (1918) S.98.

[60] Deges, Johannes: Dissertation in Vorbereitung, Max-Planck-Institut für Eisenforschung GmbH, Düsseldorf (2011).

[61] A. Mottura, N. Warnken, M.K. Miller, M.W. Finnis, u. a.: Atom probe tomography analysis of the distribution of rhenium in nickel alloys, Acta Materialia. 58 (2010) S.931-942.

[62] F. Vurpillot, A. Bostel, E. Cadel, D. Blavette: The spatial resolution of 3D atom probe in the investigation of single-phase materials, Ultramicroscopy. 84 (2000) S.213-224.

[63] T. Boll, T. Al-Kassab: Interpretation of atom probe data for the intermetallic TiAl+Nb by means of field evaporation simulation, Ultramicroscopy. eingereicht (2010). 
[64] D.G. Brandon: The field evaporation of dilute alloys, Surface Science. 5 (1966) S.137-146.

[65] T. Rademacher, T. Al-Kassab, J. Deges, R. Kirchheim: Ordering and site occupancy of $\mathrm{DO}_{3}$ ordered $\mathrm{Fe}_{3} \mathrm{Al}-5 \mathrm{at} \% \mathrm{Cr}$ evaluated by means of atom probe tomography, Ultramicroscopy. in Druck (2011).

[66] E.W. Müller: Field Desorption, Phys. Rev. 102 (1956) S.618.

[67] R.G. Forbes: Charge hopping and charge draining: Two mechanisms of field desorption, Surface Science. 102 (1981) S.255-263.

[68] R. Gomer, L.W. Swanson: Theory of Field Desorption, The Journal of Chemical Physics. 38 (1963) S.1613.

[69] N. Shima, M. Tsukada: Microscopic theory of the field induced desorption, Surface Science. 194 (1988) S.312-332.

[70] M.W. Finnis, J.E. Sinclair: A simple empirical N-body potential for transition metals, Philosophical Magazine A. 50 (1984) S.45.

[71] R. Johnson, D.J. Oh: Analytic embedded atom method model for bcc metals, Journal of Materials Research. 4 (1989) S.1195-1201.

[72] O. Yifang, Z. Bangwei, L. Shuzhi, J. Zhanpeng: A simple analytical EAM model for bcc metals including Cr and its application, Zeitschrift Für Physik B Condensed Matter. 101 (1996) S.161168.

[73] J. Cowley: Derivation of structural information from absorption effects in X-ray diffraction, Acta Crystallographica. 17 (1964) S.33-\&.

[74] J. Spence, J. Tafto: ALCHEMI - A new technique for locating atoms in small crystals, Journal of Microscopy. 130 (1983) S.147-154.

[75] C. Liebscher: Phasenanalyse im Transmissions-Elektronen-Mikroskop, Vortrag, Universität Bayreuth(2008).

[76] C. Prietzel: Messungen und Simulationsrechnungen der orientierungsabhängigen Röntgenemission an einem einkristallinen San Carlos Olivin, (1999).

[77] J. Weng, F. Zhu, J. Xiao: FIM study of $\mathrm{DO}_{3}$-ordered $\mathrm{Fe}_{3} \mathrm{Al}$, Zeitschrift Für Metallkunde. 85 (1994) S.432-436.

[78] H.J. Krause-Habrock, G. Frommeyer, J.E. Wittig, M. Kreuss: Analysis of the $\mathrm{DO}_{3}$ superlattice in ordered iron aluminium alloys by FIM and Atom Probe, Le Journal De Physique Colloques. 49 (1988) S.365-371.

[79] X.-J. Ge, N.-X. Chen, W.-Q. Zhang, F.-W. Zhu: Selective field evaporation in field-ion microscopy for ordered alloys, J. Appl. Phys. 85 (1999) S.3488.

[80] Z. Yang, R. Johnson: An EAM simulation of the alpha-gamma iron interface, Modelling and Simulation in Materials Science and Engineering. 1 (1993) S.707-716.

[81] M. Ruda, D. Farkas, J. Abriata: Embedded-atom interatomic potentials for hydrogen in metals and intermetallic alloys, Physical Review B. 54 (1996) S.9765.

[82] R. Besson, J. Morillo: Development of a semiempirical n-body noncentral potential for Fe-Al alloys, Physical Review B. 55 (1997) S.193.

[83] X.L. Shu, W.Y. Hu, H.N. Xiao, B.W. Zhang: Properties of point defects and diffusion of the B2 FeAl alloy calculated by a modified analytic EAM model, Zeitschrift Fur Metallkunde. 91 (2000) S.734738.

[84] W. Hu, X. Shu, B. Zhang: Point-defect properties in body-centered cubic transition metals with analytic EAM interatomic potentials, Computational Materials Science. 23 (2002) S.175-189. 
[85] J. Wallenius, P. Olsson, C. Lagerstedt, N. Sandberg, u. a.: Modeling of chromium precipitation in Fe-Cr alloys, Physical Review B. 69 (2004) S.094103.

[86] M.I. Mendelev, S. Han, W. Son, G.J. Ackland, u. a.: Simulation of the interaction between Fe impurities and point defects in V, Physical Review B. 76 (2007) S.214105.

[87] X.-J. Yuan, N.-X. Chen, J. Shen, W. Hu: Embedded-atom-method interatomic potentials from lattice inversion, J. Phys.: Condens. Matter. 22 (2010) S.375503.

[88] R.R. Zope, Y. Mishin: Interatomic potentials for atomistic simulations of the Ti-Al system, Phys. Rev. B. 68 (2003) S.024102.

[89] R.-Y. Li, J.-Y. Qin, T.-K. Gu, X.-F. Bian: Structure of liquid $\mathrm{Al}_{80} \mathrm{Mn}_{20}$ alloy by Reverse Monte Carlo simulation, Journal of Non-Crystalline Solids. 354 (2008) S.1736-1739.

[90] D.D. von Bezold: Mikrokanalplatte für höhere Frequenzen, http://www.patentde.com/19880414/DE3733101A1.html, Zugriff am 30. März 2011.

[91] D. Liu, R. Wang, Y. YE: Stability comparison of several icosehedral structure units of Al-Cr alloys, Physical Review B. 43 (1991) S.4648-4652.

[92] Y.-M. Kim, Y.-H. Shin, B.-J. Lee: Modified embedded-atom method interatomic potentials for pure $\mathrm{Mn}$ and the Fe-Mn system, Acta Materialia. 57 (2009) S.474-482.

[93] P. Qian, N.-X. Chen, J. Shen: Atomistic simulation on site preference and lattice vibrations of $\mathrm{NdMn}_{8} \mathrm{~T}_{4}(\mathrm{~T}=\mathrm{Fe}, \mathrm{Co}, \mathrm{Ni})$, J. Phys. D: Appl. Phys. 38 (2005) S.1199-1204.

[94] T. Kresse: Untersuchung der Platzbesetzung von Titan in $\mathrm{DO}_{3}$ geordneten Fe-26at.\%Al, Diplomarbeit, Georg-August-Universität Göttingen(2009).

[95] W.Y. Hu, B.W. Zhang, B.Y. Huang, F. Gao, u. a.: Analytic modified embedded atom potentials for HCP metals, Journal of Physics-condensed Matter. 13 (2001) S.1193-1213.

[96] T. Boll: Bestimmung von Platzbesetzung und Bindungsenergien mittels Atomsondentomographie, Dissertation, Georg-August-Universität Göttingen(2010).

[97] I. Sa, B.-J. Lee: Modified embedded-atom method interatomic potentials for the Fe-Nb and $\mathrm{Fe}-\mathrm{Ti}$ binary systems, Scripta Materialia. 59 (2008) S.595-598.

[98] D. Farkas: interatomic potentials for Ti-Al with and without angular forces, Modelling and Simulation in Materials Science and Engineering. 2 (1994) S.975-984.

[99] E. Marquis, F. Vurpillot: Chromatic Aberrations in the Field Evaporation Behaviorof Small Precipitates, Microscopy and Microanalysis. 14 (2008) S.561-570.

[100] F. Vurpillot, A. Bostel, D. Blavette: Trajectory overlaps and local magnification in threedimensional atom probe, Appl. Phys. Lett. 76 (2000) S.3127.

[101] X. Sauvage, L. Renaud, B. Deconihout, D. Blavette, u. a.: Solid state amorphization in cold drawn $\mathrm{Cu} / \mathrm{Nb}$ wires, Acta Materialia. 49 (2001) S.389-394.

[102] F. De Geuser, W. Lefebvre, F. Danoix, F. Vurpillot, u. a.: An improved reconstruction procedure for the correction of local magnification effects in three-dimensional atom-probe, Surf. Interface Anal. 39 (2007) S.268-272.

[103] G.J. Ackland, G. Tichy, V. Vitek, M.W. Finnis: Simple N-body potentials for the noble metals and nickel, Philosophical Magazine A. 56 (1987) S.735.

[104] H. Teichler: private Korrespondenz, (2010).

[105] T. Maruyama, Y. Hasegawa, T. Nishi, T. Sakurai: FIM and Atom-probe study of polymers, Le Journal De Physique Colloques. 48 (1987) S.6.

[106] C. Nowak: Electric Field influence on Low Temperature Metal Oxidation, Dissertation, GeorgAugust-Universität Göttingen(2008). 
[107] M.J. Marcinkowski, N. Brown: Direct Observation of Antiphase Boundaries in the $\mathrm{Fe}_{3} \mathrm{Al}$ Superlattice, Journal of Applied Physics. 33 (1962) S.537 - 552.

[108] C. Kluthe: Wachstum und Grenzflächenbeschaffenheit oxidischer Ausscheidungen in Silber, Dissertation, Georg-August-Universität Göttingen(2003).

[109] L. Toth: Transition metal carbides and nitrides, Academic Press, New York (1971).

[110] E. Storms: The refractory carbides, Academic Press, New York [u.a.] (1967).

[111] S.V. Meschel, O.J. Kleppa: Standard enthalpies of formation of some $4 d$ transition metal silicides by high temperature direct synthesis calorimetry, Journal of Alloys and Compounds. 274 (1998) S.193-200.

[112] K.M. Krishnan, P. Rez, G. Thomas: Crystallographic site-occupancy refinements in thin-film oxides by channelling-enhanced microanalysis, Acta Crystallographica Section B. 41 (1985) S.396-405.

[113] R.W. Cahn, P. Haasen, (Eds): Physical Metallurgy Part II, Third Edition, North-Holland Physics Publishing (1983).

[114] G.D. Loper, L.C. Smedskjaer, M.K. Chason, R.W. Siegel: Determination of the vacancy formation enthalpy in chromium by positron annihilation, Argonne National Laboratory, USA, Materials Science and Technology Division (1985).

[115] T. Korhonen, M.J. Puska, R.M. Nieminen: Vacancy-formation energies for fcc and bcc transition metals, Phys. Rev. B. 51 (1995) S.9526.

[116] P.A. Korzhavyi, I.A. Abrikosov, B. Johansson, A.V. Ruban, u. a.: First-principles calculations of the vacancy formation energy in transition and noble metals, Phys. Rev. B. 59 (1999) S.11693.

[117] H.-E. Schaefer, R. Gugelmeier, M. Schmolz, A. Seeger: Positron Lifetime Spectroscopy and Trapping at Vacancies in Aluminium, Mater. Sci. Forum. 1 (1987) S.111-116.

[118] N. Ashcroft: Festkörperphysik, Oldenbourg, München [u.a.] (2001). 


\section{Abbildungsverzeichnis}

Abbildung 2.1: Phasendiagramm Eisen-Aluminium (nach MASSALSKI [10]) ..........................................

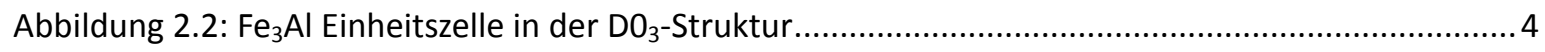

Abbildung 2.3: Temperaturabhängigkeit der kritischen Scherspannung …............................................ 4

Abbildung 3.1: Schematischer Aufbau und Funktionsweise von FIM- und APT-Messungen ....................11

Abbildung 3.2: Geometrische Verhältnisse der Rekonstruktion von Atompositionen bei der APT ..........13

Abbildung 3.3: Fertig präparierte Lamelle für TEM-/ALCHEMI-Untersuchungen. ....................................16

Abbildung 3.4: XRD-Spektren nanokristalliner Proben nach 8h Mahldauer .............................................18

Abbildung 3.5: Präparation von APT-Proben mit dem FIB. Erläuterungen siehe Text..............................19

Abbildung 3.6: Schematische Potentialverläufe eines evaporierenden Atoms nach dem Image-Hump-

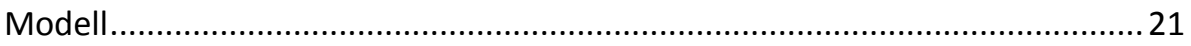

Abbildung 3.7: Potentialverläufe eines evaporierenden Atoms nach dem Charge-Exchange-Modell....22

Abbildung 3.8: Aufsicht auf simulierte Probenspitze: Energetischer Einfluss des Feldes. .......................22

Abbildung 3.9: Schematische Darstellung der Form der virtuellen APT-Spitze .......................................25

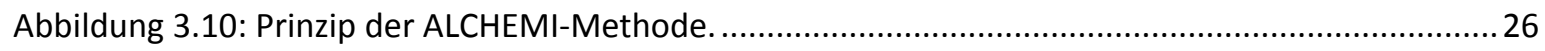

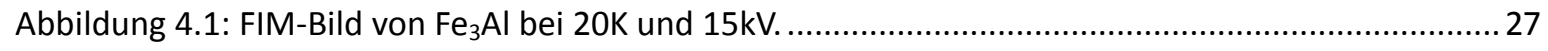

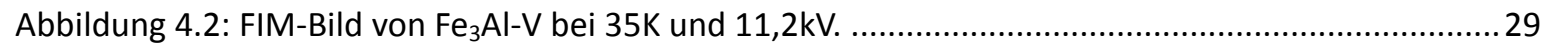

Abbildung 4.3: Ausschnitt aus einem mit der APT gemessenen Volumen..............................................30

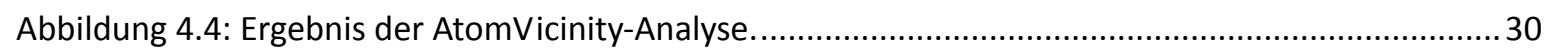

Abbildung 4.5: Übersicht der Abfolge von Ebenen und entsprechende Dichteprofile ............................ 31

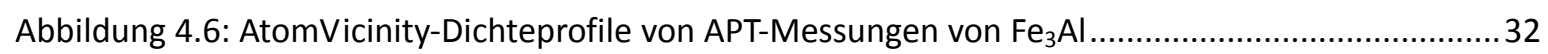

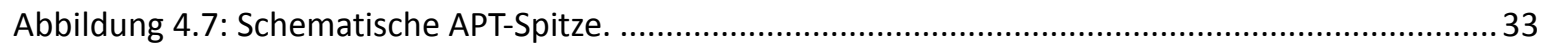

Abbildung 4.8: Paarpotentiale der binären Legierung ……….............................................................. 36

Abbildung 4.9: AtomVicinity-Dichteprofile einer simulierten APT-Messung von $\mathrm{Fe}_{3} \mathrm{Al}$..........................37

Abbildung 4.10: AtomVicinity-Dichteprofile von Aluminium: Messung und Simulationen mit

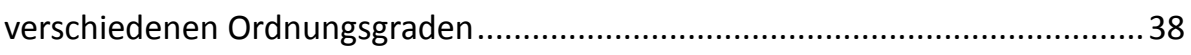

Abbildung 4.11: Einfluss von $r_{k}$ und $r_{s}$ auf die Rekonstruktion des simulierten APT-Volumens ...............42

Abbildung 5.1: AtomVicinity-Dichteprofile von APT-Messungen von $\mathrm{Fe}_{3} \mathrm{Al}-\mathrm{Cr}$........................................4 44

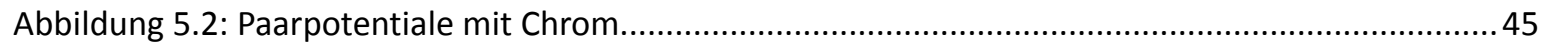

Abbildung 5.3: AtomVicinity-Dichteprofile simulierter APT-Messungen von $\mathrm{Fe}_{3} \mathrm{Al}-\mathrm{Cr}$ mit $\beta$ -

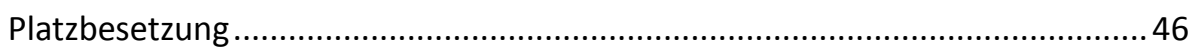

Abbildung 5.4: AtomVicinity-Dichteprofil simulierter APT-Messungen mit Chrom ................................ 47

Abbildung 5.5: AtomVicinity-Dichteprofile von APT-Messungen von $\mathrm{Fe}_{3} \mathrm{Al}-\mathrm{Mn}$..................................... 48

Abbildung 5.6: Paarpotentiale mit Mangan ............................................................................................. 49 
Abbildung 5.7: AtomVicinity-Dichteprofile simulierter APT-Messungen von $\mathrm{Fe}_{3} \mathrm{Al}-\mathrm{Mn}$ mit $\beta$-Platzbesetzung .............................................................................. 50

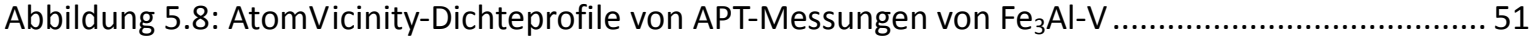

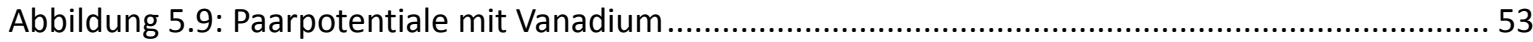

Abbildung 5.10: AtomVicinity-Dichteprofile einer simulierten APT-Messung von $\mathrm{Fe}_{3} \mathrm{Al}-\mathrm{V}$ mit

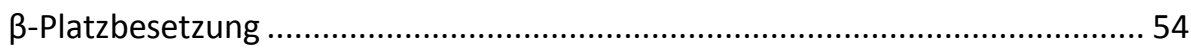

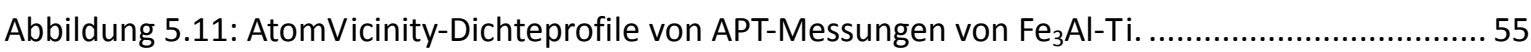

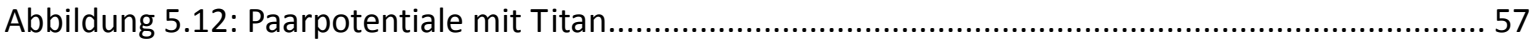

Abbildung 5.13: AtomVicinity-Dichteprofile simulierter APT-Messungen von Fe ${ }_{3} \mathrm{Al}-\mathrm{Ti}$ mit $\beta$-Platzbesetzung 58

Abbildung 5.14: Al-Ti-Paarpotential mit zwei unterschiedlichen Positionen des Minimums 59

Abbildung 5.15: AtomVicinity-Profile simulierter APT-Messungen an Fe ${ }_{3} \mathrm{Al}-\mathrm{Ti}$ mit $\alpha$-Platzbesetzung .... 60

Abbildung 7.1: Dunkelfeld-TEM-Aufnahmen von $\mathrm{Fe}_{3} \mathrm{Al}-\mathrm{Cr}$ (Charge 3)..... 63

Abbildung 7.2: TEM-Beugungsbild von nanokristallinem Fe $\mathrm{Al}_{3} \mathrm{Ti}$ (Charge 6) ..................................6.6. 64

Abbildung 7.3: REM-Aufnahmen von APT-Spitzen nach erfolglosen Messungen:..............................66

Abbildung 7.4: APT-Rekonstruktion von nanokristallinem $\mathrm{Fe}_{3} \mathrm{Al}-\mathrm{Cr}$ (Charge 1) ................................66 66

Abbildung 7.5: Konzentrationsverläufe von nanokristallinem $\mathrm{Fe}_{3} \mathrm{Al}-\mathrm{Cr}$........................................67

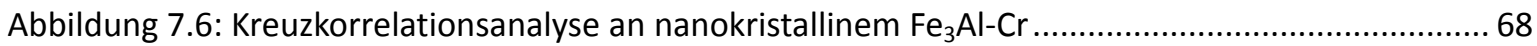

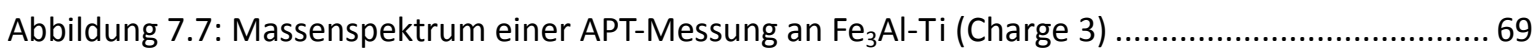

Abbildung 7.8: Nach Ionen getrennte Rekonstruktion einer APT-Messung von Fe $\mathrm{Al}_{3} \mathrm{Ai}$.......................70

Abbildung 7.9: Eindimensionaler Konzentrations- und Dichteverlauf von Fe $\mathrm{Al}_{3} \mathrm{Al}$ (Charge 2) ............. 71

Abbildung 7.10: Kreuzkorrelationsanalyse von nanokristallinem Fe ${ }_{3} \mathrm{Al}-\mathrm{Ti}$ (Charge 2)......................... 73

Abbildung 7.11: Ausschnitte aus rekonstruiertem APT-Volumen von Fe $\mathrm{Al}-\mathrm{Cr}$ (Charge 3)................... 73

Abbildung 7.12: Eindimensionaler Dichteverlauf in nanokristallinem $\mathrm{Fe}_{3} \mathrm{Al}-\mathrm{Cr}$ (Charge 3) .................. 74

Abbildung 7.13: Kreuzkorrelationsanalyse von nanokristallinem $\mathrm{Fe}_{3} \mathrm{Al}-\mathrm{Cr}$ (Charge 3) ..........................75

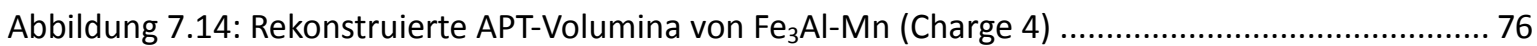

Abbildung 7.15: Kreuzkorrelationsanalyse von nanokristallinem Fe $\mathrm{Al}_{3} \mathrm{Mn}$ (Charge 4) ...................... 77

Abbildung 7.16: Rekonstruierte APT-Volumina von Fe 3 Al-V (Charge 5) .......................................... 78

Abbildung 7.17: Kreuzkorrelationsanalyse von nanokristallinem $\mathrm{Fe}_{3} \mathrm{Al}-\mathrm{V}$ (Charge 5).......................... 79

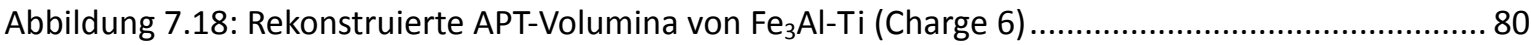

Abbildung 7.19: Eindimensionaler Dichteverlauf in nanokristallinem $\mathrm{Fe}_{3} \mathrm{Al}-\mathrm{Ti}$ (Charge 6)...................80

Abbildung 7.20: Kreuzkorrelationsanalyse von nanokristallinem Fe ${ }_{3} \mathrm{Al}-\mathrm{Ti}$ (Charge 6) ..........................82

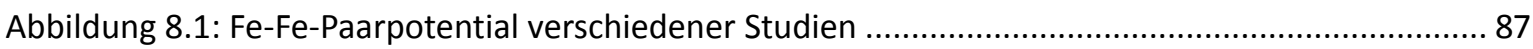

Abbildung 8.2: Schematische Beziehung der Energiebeiträge der Feldevaporation ...........................88

Abbildung 8.3: Ordnungsgrade in Fe ${ }_{3} \mathrm{Al}$ verschiedener Studien .................................................. 91 
Abbildung 8.4: Schematische Feldevaporationsartefakte an einer APT-Spitze. ......................................93

Abbildung 8.5: Kohlen- und Sauerstoffkonzentration in Abhängigkeit der Mahldauer ............................94

Abbildung 8.6: Segregationsindizes nanokristalliner Proben ..............................................................95

Abbildung 8.7: Übersicht der Kreuzkorrelationsanalysen an nanokristallinem ternären $\mathrm{Fe}_{3} \mathrm{Al}$.............95

Abbildung A.1: Berechnete relative EDX-Intensitäten abhängig vom Verkippungswinkel........................IX

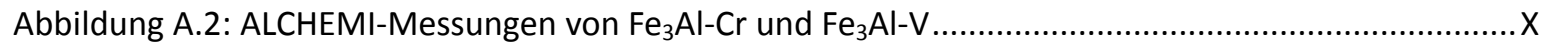

Abbildung C.1: Geometrische Verhältnisse der Projektion bei der APT...................................................II 


\section{Akademischer Lebenslauf}

Vollständiger Name: $\quad$ Thomas Jürgen Rademacher

Geboren am:

20. Juli 1980

Geboren in:

Lehrte

Staatsangehörigkeit:

Deutsch

30.6 .2000

Erlangen der allgemeinen Hochschulreife am Gymnasium Burgdorf

1.10.2001 - 30.9.2007

Studium der Physik an der Georg-August-Universität Göttingen

23.10.2003

Vordiplom im Fach Physik

19.7.2007

Diplom im Fach Physik

Thema der Diplomarbeit: „Zum Einfluss der elastischen Verzerrung auf die Frühstadien der Entmischung in $\mathrm{Cu}-1,7 \mathrm{at} . \% \mathrm{Fe}^{\prime \prime}$

1.10.2007 - 30.9.2011

Promotionsstudium der Physik an der Georg-August-Universität Göttingen

13.7.2011

Disputation an der Georg-August-Universität Göttingen 


\section{Danksagung}

Das Verfassen dieser Arbeit und die Durchführung der entsprechenden Studien waren nur möglich durch die Mithilfe einer Vielzahl von Menschen, bei denen ich mich für ihr Engagement und ihre Hilfsbereitschaft bedanken möchte.

Als erstes danke ich Herrn Prof. Dr. R. Kirchheim und Herrn Prof. Dr. T. Al-Kassab für die Möglichkeit des Absolvierens meiner Promotion am Institut für Materialphysik Göttingen, der Betreuung während dieser Zeit und der steten fachlichen Unterstützung. Bedanken möchte ich mich bei Herrn Al-Kassab auch für die Gelegenheit zu dem Forschungsaufenthalt an der King Abdullah University in Saudi-Arabien.

Für die Finanzierung dieser Arbeit im Rahmen des Projektes AL 592/3-1 sei der Deutschen Forschungsgemeinschaft gedankt; für die erfolgreiche Zusammenarbeit innerhalb dieses Projekts und die Herstellung der Proben danke ich Herrn J. Deges und Herrn Prof. Dr. Frommeyer des Max-Plack-Instituts für Eisenforschung, Düsseldorf.

Herrn Dr. T. Boll danke ich für fachliche Unterstützung und insbesondere für die Hilfe bei der Durchführung von APT-Messungen an der KAUST.

Bei Herrn M.Sc. M. Sobol bedanke ich mich ausdrücklich für die Unterstützung der programmiertechnischen Aufgaben, insbesondere bei den Simulationsrechnungen.

Für die Hilfe und das Engagement bei der Durchführung der ALCHEMI-Messungen danke ich Herrn H. Schumann und Herrn Prof. Dr. M. Seibt des IV. Physikalischen Instituts der Uni Göttingen sowie Herrn D. Huber und Herrn Prof. Dr. H. Fraser der COEF der Ohio State University, USA.

Für die Hilfsbereitschaft, fachlichen Beistand und technische Unterstützung danke ich Herrn Dr. C. Nowak, Frau Dr. C. Wille, Herrn T. Schulz, Herrn Dipl. Phys. T. Kresse, Herrn Prof. Dr. H. Teichler, Herrn Dr. P.J. Wilbrandt und Herrn V. Radisch. Außerdem gilt dieser Dank auch allen Mitgliedern des IMP der Uni Göttingen für ihre Hilfsbereitschaft und ein stets angenehmes Arbeitsklima.

Für Korrekturhilfe an dieser Arbeit und weitere Unterstützung danke ich Kerstin und Heike.

Außerdem danke ich für weitere, außerfachliche Hilfestellung meinen Freunden und meiner Familie, insbesondere danke ich meinen Eltern, Elisabeth Rademacher und Günther Rademacher, für die Unterstützung und das Ermöglichen meiner Promotion.

Thomas Rademacher 